NASA Contractor Report 3371

\title{
Refraction and Scattering of Sound by a Shear Layer
}

Robert H. Schlinker and Roy K. Amiet

United Technologies Research Center

East Hartford, Connecticut

Prepared for Langley Research Center under Contract NAS1-15339

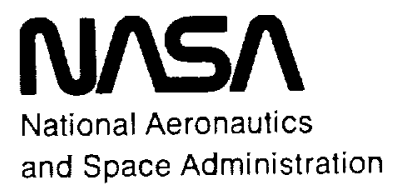

Scientific and Technical Information Branch 

Previous Theoretical Investigations . . . . . . . . . . . . . 4

Previous Experimental Investigations . . . . . . . . . . . . 5

Method of Approach . . . . . . . . . . . . . . . . 6

LIST OF SYMBOLS . . . . . . . . . . . . . . . . . . . . . 7

DESCRIPTION OF THE EXPERIMENT . . . . . . . . . . . . . . . . 12

Acoustic Research Tunnel . . . . . . . . . . . . . . . 12

Experimental Arrangement . . . . . . . . . . . . . . . 16

Instrumentation . . . . . . . . . . . . . . . . . 20

Test Program . . . . . . . . . . . . . . . . . . 21

DEFINITION OF THE OPEN JET SHEAR LAYER . . . . . . . . . . . . . . . 24

Mean Velocity Profiles . . . . . . . . . . . . . . 24

Shear Layer Thickness . . . . . . . . . . . . . . . . . 25

Similarity of Mean Velocity Profiles . . . . . . . . . . . 26

Turbulence Intensity . . . . . . . . . . . . . . . 26

THEORETICAL FORMULATION OF THE REFRACTION PROBLEM . . . . . . . . . . 28

Review . . . . . . . . . . . . . . . . . . 28

Shear Layer Correction For an Off-Axis Acoustic Source . . . . . 29

Theoretical Refraction Predictions for Comparison With Experiment • 30

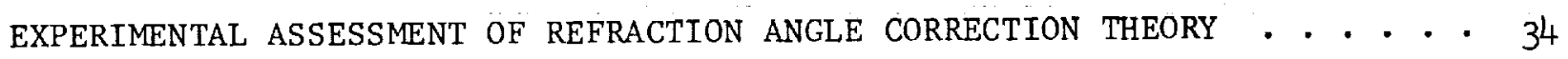

Experimental Approach . . . . . . . . . . . . . . . . 34

Measured Phase Difference and Calculated Angle Change . . . . . . 34

Background Noise and Non-Anechoic Effects . . . . . . . . . . 36

Plane Wave Propagation Assumptions . . . . . . . . . . . . . 38

Comparison of Measured and Theoretical Refraction Angle

Correction ....................... 40

Summary and Evaluation ................. . . 41 
EXPERIMENTAL ASSESSMENT OF REFRACTION AMPLITUDE CORRECTION THEORY • • • 43

Experimental Approach . . . . . . . . . . . . . . . . 43

Calculating the Refraction Amplitude Correction from Measured Sound

Pressure Levels . . . . . . . . . . . . . . . 44

Nose Cone Directional Sensitivity Effects . . . . . . . . . . . . 47

Comparison of Measured and Theoretical Refraction Amplitude

Correction . . . . . . . . . . . . . . . . . 48

Comparisons With Other Investigators . . . . . . . . . . . 53

Sumary and Evaluation . . . . . . . . . . . . . . . 55

TURBULENCE SCATTERING OF SOUND EXPERIMENTS . . . . . . . . . . . . . 56

Formulation of the Problem . . . . . . . . . . . . . . 56

Parameters Controlling Scattering . . . . . . . . . . . . . 57

Discussion of Experimental Results . . . . . . . . . . . . . 59

Prediction of Turbulent Scattering Effects . . . . . . . . . . 63

CONCLUSTONS . . . . . . . . . . . . . . . . . . . . . . . . 67

APPENDIX A - DERIVATION OF SHEAR LAYER CORRECTION FOR AN OFF-AXIS

ACOUSTIC SOURCE.................... 69

APPENDIX B - WAVEFRONT ANGLE CHANGE CALCULATION . . . . . . . . . . 84

APPENDIX C - SHEAR LAYER ANGLE CORRECTION CALCULATION . . . . . . . . 85

APPENDIX D - MICROPHONE-ACOUSTIC DRIVER CROSS-CORRELATION

TECHNIQUE . . . . . . . . . . . . . . . . . . . 86

APPENDIX E - CROSS-CORRELATION TECHNIQUE IN PRESENCE OF REFLECTED

SOUND WAVES . . . . . . . . . . . . . . . . 89

APPENDIX F - ERROR ANALYSIS FOR SHEAR LAYER ANGLE CORRECTION EXPERIMENT . . . . . . . . . . . . . . . . 92

REFERENCES . . . . . . . . . . . . . . . . . . . . . . . . 93

TABLES . . . . . . . . . . . . . . . . . . . . . . . . . 95

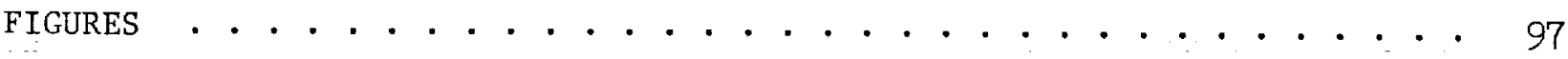




\section{SUMMARY}

A theoretical and experimental investigation was conducted to determine the effect of refraction and turbulence scattering on sound transmission through a circular, open-jet shear layer. Experiments were performed using a $0.91 \mathrm{~m}$ diameter open jet in the United Technologies Research Center (UTRC) Acoustic Research Tunnel. Free stream Mach number was varied from 0.1 to 0.4 .

For measurements directed toward assessment of the refraction angle and amplitude change, a discrete tone acoustic source with a frequency range of $1 \mathrm{kHz}$ to $10 \mathrm{kHz}$, was situated in the airstream. Sound wavefront angle and amplitude changes across the shear layer were measured for several axial source locations and two off-axis source positions. Far-field noise directivity patterns were significantly altered at test Mach numbers of 0.1 and greater due to refraction by the open-jet shear layer. Experimental results were compared with an existing refraction theory which was extended in the present study to account for off-axis source positions.

Good agreement between refraction theory and experiment was obtained over the test Mach number, frequency, and angle measurement range for all on-axis acoustic source locations. For the range of open-jet shear layer thicknesses investigated by changing the source location, the refraction angle and amplitude changes were independent of shear layer thickness. This independence of shear layer thickness and divergence confirms the zero thickness shear layer model used in the theoretical prediction.

The refraction angle and amplitude changes were independent of frequency over the frequency range considered in the present experiment. This independence confirms the theoretical prediction. Angle and amplitude changes across the shear layer can, therefore, be corrected for using the experimentally verified refraction theory. A general correction procedure is, thus, available for reducing far-field noise data acquired in open-jet test facilities.

The generalized refraction theory predicted large differences between on-axis and off-axis corrections. Good agreement between theory and experiment was obtained at a source-to-shear layer separation distance greater than the jet radius. Mach number dependence and frequency independence were confirmed. Measurable differences between theory and experiment occurred at a source-toshear layer separation distance less than one jet radius. This disagreement is at present not understood. 
An experiment was also conducted to evaluate the effect of open-jet shear layer turbulence scattering on discrete tone propagation. Frequencies varying over the range of $5 \mathrm{kHz}$ to $15 \mathrm{kHz}$ were investigated as the Mach number varied from 0.1 to 0.3 . Attenuation of the discrete tone amplitude and the resulting tone broadening were measured. These features were found to be stronger at angles close to the open-jet axis than at $90^{\circ}$. More severe scattering was also observed for downstream source locations where the ratio of shear layer propagation path length to acoustic wave length approached a value of 10 .

In addition to the experimental effort, an existing single-scattering analysis was modified to provide an estimate for the operating conditions at which the onset of scattering occurs and the resulting discrete tone amplitude attenuation. Experimental results showed reasonable agreement. 
Open jet acoustic test facilities are currently used to investigate the effect of forward flight on aeroacoustic noise mechanisms. The technique permits studying a wide variety of problems such as airframe noise, model propeller and rotor noise, isolated airfoil and blown flap noise, and jet noise forward flight effects. For tests conducted at free stream Mach numbers less than 0.1 , measurements outside the airstream can be used to directly infer the source noise characteristics. However, at higher Mach numbers, the open jet technique is influenced by the presence of the shear layer through which the sound is transmitted. The shear layer serves to refract, reflect, and scatter the sound radiated from the model. These effects significantly alter the acoustic source directivity pattern and hence alter the conclusions drawn from a particular experiment.

The lack of a firm understanding of these effects created a clear need for a validated shear layer correction procedure. The present study was undertaken to experimentally assess the refraction angle and amplitude changes due to propagation through a finite thickness shear layer. Both on-axis and off-axis acoustic source locations were tested for a circular jet geometry. Acoustic source frequency and jet Mach number were also varied to evaluate the dependence on these parameters. In addition to the experimental effort, an existing refraction theory was extended to treat the case of a general off-axis source location. If the experiment verified the theory, a general correction procedure would be available for reducing the far-field noise data acquired in open jet test facilities.

These objectives were pursued in two separate but sequential research programs. The first program (ref. 1) investigated the refraction angle change and found good agreement between theory and experiment. A small effort was also devoted to the refraction amplitude change but the theory could not be verified due to limitations of the experimental technique. A second research program was therefore conducted to provide a definitive experimental assessment of the refraction amplitude correction theory. Results of this study are presented in this report.

Since the refraction angle change across the shear layer was needed to determine the far-field microphone location in the amplitude correction experiment, the results of the first research program were critical to conducting the second study. For this reason, the refraction angle correction experimental results are also included in this report. Refraction theory for the general off-axis source position is also included. Thus, this report provides a complete theoretical solution to the refraction problem in addition to the experimental verification of the theory. This theoretical correction procedure can now be used to analyze far-field noise data acquired in open-jet acoustic wind tunnels. A listing of the 
computer program for predicting the refraction angle and amplitude corrections is given in the appendix.

An experiment was also conducted to evaluate the effect of open jet shear layer turbulence scattering on discrete tone propagation. While this scattering phenomenon has been found to be unimportant for acoustic wind tunnel tests of broadband noise sources, it may be important for studies in which discrete tones are generated. Examples of such studies include model propeller and helicopter rotor noise, turbofan noise, and supersonic jetscreech noise. The magnitude of turbulence scattering in each case is a function of the discrete tone frequency, the open jet Mach number, and the acoustic source position. In such model studies, a scattering correction may be needed to calculate absolute sound pressure levels and directivity patterns from the far-field microphone data. For these reasons, an experiment was conducted to measure the reduction in the discrete tone amplitude and the resulting tone broadening due to turbulence scattering.

A range of frequencies and Mach numbers were employed to determine when scattering becomes significant. The importance of propagation path length through the turbulent shear layer and the turbulence length scale were investigated by locating the acoustic source at various axial stations. These parameters were also evaluated as a function of acoustic radiation angle relative to the open jet axis.

In addition to the experimental effort an existing single scattering analysis was modified to provide an estimate of the discrete tone amplitude attenuation. The modified analysis was also used to derive an expression for predicting the onset of turbulence scattering. Experimental results were compared with calculations from the modified analysis. Predictions from an existing multiple scattering analysis were also compared with the experimental data.

\section{Previous Theoretical Investigations}

Because the use of open jet wind tunnels for determining the acoustic radiation properties of test models is a relatively new technique, methods have only recently become avaliable for correcting the acoustic data obtained in such a facility for the effects of refraction. However, the refraction problem had received previous attention because of its importance in the jet noise problem. The case of refraction of a plane sound wave by a plane, zero thickness shear layer, was first correctly treated by Ribner (ref. 2) and Miles (ref. 3). Gottlieb (ref. 4) extended the analysis to the case of a point source beneath a plane shear layer. Graham and Graham (ref. 5) considered the problem of a plane wave interacting with a finite thickness shear layer and subsequent publications of theirs consider the field of specific singularities near a shear layer. Amiet (ref. 6) and others also considered the sound field of specific source types near a shear layer. 
The above studies were not concerned with correcting acoustic wind tunnel data, however, and further analysis of the problem was needed. In particular, a method was required which made no assumptions regarding the nature of the sources (e.g., monopole, dipole, etc.), since, in general, the nature and distribution of sources in a given test was unknown. Subsequently, Amiet (ref. 7) derived a correction procedure which applied both an angle change and an amplitude change to the data. This assumed a plane zero thickness shear layer. It was only by this separation of angle and amplitude effects that it was possible to arrive at a correction independent of source type. The problem was also analyzed by Jacques (ref. 8) who arrived at the same result for refraction by a plane shear layer, and also considered the case of a source on the centerline of a cylindrical shear layer of zero thickness. The solution for refraction by a thick cylindrical shear layer with a source on the centerline was given by Tester and Morfey (ref. 9) and for a thick plane shear layer by Amiet (ref. 10). This work of Amiet also gives a thorough review of the several correction procedures.

The above corrections are in algebraic closed form. Candell (ref. 11) recently developed a numerical ray tracing procedure which appears to give results very close to the closed form solutions. Recent studies by Tester and Burrin (ref. 12) indicate that the axial variation of the shear layer has little effect on the refraction corrections. Thus, it is possible to model the open jet shear layer without axial variations.

References 7-12 rely on the technique of correcting both angle and amplitude. Mani (ref. 13) employed a different approach to the problem, but this appears to be less well established than the above mentioned techniques of correcting angle and amplitude.

\section{Previous Experimental Investigations}

Few experimental studies exist documenting the shear layer refraction and scattering characteristics. Amiet (ref. 7) reported the results of an exploratory experiment for the angle correction using a plane shear layer. The results tended to support the theoretical predictions presented in the same study. Candell et al. (ref. 14) provided an experimental assessment of a refraction theory for a circular jet. In addition they presented preliminary results for turbulence scattering by the jet shear layer. Similarly, Ahuja et al. (ref. 15) recently reported an assessment of the refraction theory of reference 12 for a circular jet. Also included was an exploratory study of turbulence scattering at low jet Mach numbers and low acoustic source frequencies. Ozkul and Yu (ref. 16) investigated the angle and amplitude changes in a circular jet and compared the results to the refraction theory given by Amiet in reference 7 . A11 of the above experimental investigations were limited to on-axis source locations. 
In the present study, the refraction angle change was assessed by cross-correlating far-field microphones to measure the local acoustic wavefront propagation angle outside the open jet. Knowing the propagation angle, the acoustic ray normal to the wavefront was traced back to the shear layer crossing point. In determining this point, the shear layer was assumed to be cylindrical with a radius equal to the inlet nozzle radius and to have zero thickness. From the acoustic ray crossing point, the original propagation angle inside the shear layer was determined for comparison with the general refraction angle correction theory developed as part of this study.

The refraction amplitude change across the shear layer was assessed by comparing acoustic source sound pressure level measurements inside and outside the open jet test section. The microphone location inside the potential core coincided with a ray propagating at a selected radiation angle. The out-of-flow or far-field microphone was situated on the same acoustic ray except that the ray was not refracted by the jet shear layer. The intersection of the refracted ray with the microphone sideline determined the far field measurement station. This intersection point was predicted analytically by the refraction angle change theory. Verification of the refraction angle change was, therefore, a prerequisite to conducting the refraction amplitude correction experiment.

The ratio of the measured in-flow to far-field microphone sound pressure level represented the amplitude change along the refracted path. This amplitude change was compared with the calculated inverse square-law amplitude change along a non-refracted ray path which intersected the same microphone sideline. The inverse square-law change corresponded to the sound pressure level change in the absence of a refracting shear layer. The decibel difference between the amplitude change on the refracted and non-refracted path represented the refraction amplitude correction. The experimentally determined amplitude correction was then compared with the theory. It should be emphasized that the refraction amplitude correction converts a sound pressure level measurement outside the open jet to a level that would be measured in the flow but in the absence of the shear layer.

The discrete tone turbulence scattering experiment was conducted using the same in-flow and far-field microphone geometry employed in the refraction amplitude correction experiment. In-flow spectra were compared with far field spectra after correcting for the refraction amplitude change and inverse square-law decay between the two microphone stations. Differences between the discrete tone amplitudes in the two spectra provided a direct calibration of signal attenuation due to scattering of sound into other propagation directions and frequencies. The significance of tone broadening was determined from the spectrum shape. Experimental results were compared with predictions from two separate scattering analyses. 
LIST OF SYMBOLS

A

a

AMP

$\mathrm{B}_{1}, \mathrm{~B}_{2}, \mathrm{~B}_{3}, \mathrm{~B}_{4}$

$\mathrm{B}_{2, \mathrm{~K}}$

$\mathrm{c}_{\mathrm{o}}$

$\mathrm{C}_{\mathrm{F}}$

$\mathrm{D} / \mathrm{Dt}$

$\mathrm{dB}$

$\mathrm{d} \ell$

E

f

$G_{1}, G_{2}$

${ }_{A, C}{ }^{\mathrm{A}}, \mathrm{B}$

g

$\mathrm{H}$

h

I
Cross-sectional area of ray tube

Radius of tunnel

Amplitude correction function

Discrete tone pressure wave amplitude measured at microphones, $\mathrm{m}_{1}, \mathrm{~m}_{2}, \mathrm{~m}_{3}, \mathrm{~m}_{4}$

Reflected wave amplitude measured at microphone $m_{2}$

Sound speed

Amplitude correction function

$\partial / \partial t+U \partial / \partial x$

Decibel

Vector defining side of ray tube

Energy scattered in new directions from a unit volume of turbulence per unit time

Acoustic source frequncy, $\mathrm{Hz}$

Microphone sensitivity

Cross-power spectral density functions

$1-\mathrm{h} / \mathrm{a}$ or $1-\mathrm{h} / \mathrm{R}_{\mathrm{o}}$

Cross-correlation amplitude

Source-to-shear layer distance. Also corresponds to sourceto-lip-line distance.

Intensity of incident sound 
LIST OF SYMBOLS (Cont'd)

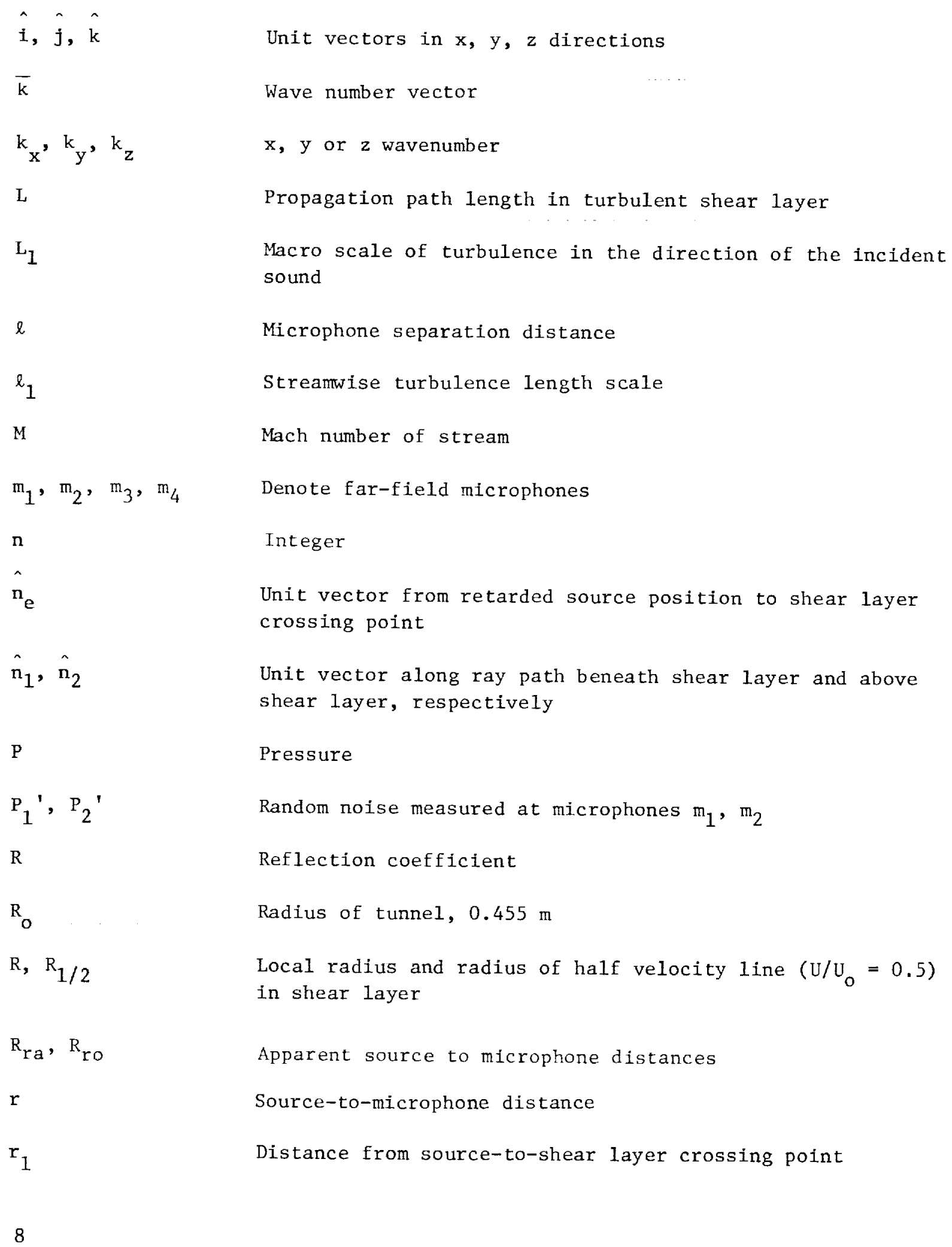




\section{LIST OF SYMBOLS (Cont'd)}

$\mathrm{r}_{2}$

$r_{3}, r_{4}$

S

$\mathrm{T}$

$\mathrm{t}$

$\mathrm{U}, \mathrm{U}_{\mathrm{o}}$

V

$\overline{\left(v_{1}^{\prime}\right)^{2}}$

w

$\mathrm{X}$

$X, Y$

$x, y, z$

$x, y^{\prime}, z^{\prime}$

$\alpha$

$\beta^{2}$

$\gamma$

$\Delta_{p}$

$\Delta_{r}$

$\Delta, \Delta^{\prime}$
Distance from shear layer crossing point to far-field microphone

Distance from source to in-flow and far-field microphone on non-refracted path ray path

Cross-correlation function

Transmission coefficient

Time

Local stream velocity in shear layer and potential core velocity

Voltage signal

Mean-square velocity of turbulence

$z$ component of perturbation velocity

Acoustic ray shear layer crossing-point

Axial and transverse coordinates in the horizontal plane coinciding with open jet axis

Cartesian coordinates rotated by a from $x, y^{\prime}, z^{\prime}$

Cartesian coordinates fixed to laboratory frame

Angle made by tangent plane

$1-\mathrm{M}^{2}$

$\varphi+a$

Phase lag between speaker output and acoustic signal arriving at microphone

Difference in distances between source and individual microphones

Phase difference between microphones due to propagation path length differences at $M=0$ and $M \neq 0$ 


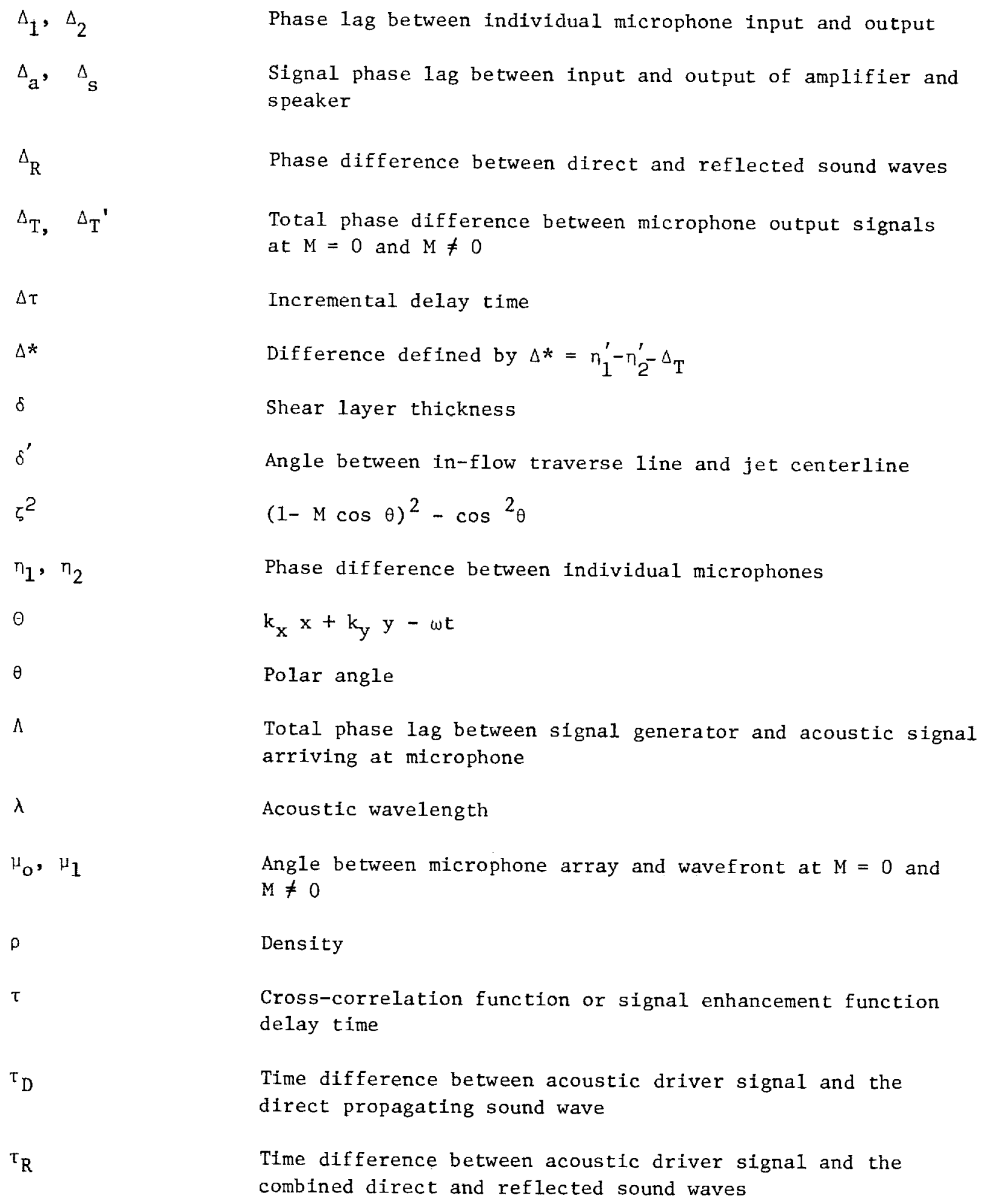


LIST OF SYMBOLS (Cont'd)

$\Phi$

$\phi$

$\omega$

$<>$

Subscripts

i

v

t

c

m
Velocity potential

Polar angle

Radian frequency

Time average of fluctuating signal

Incident

Reflected

Transmitted

Corrected

Measured 


\title{
DESCRIPTION OF THE EXPERIMENT
}

\author{
Acoustic Research Tunnel
}

Operating Characteristics - The experimental study was conducted in the UTRC Acoustic Research Tunnel. A detailed description of the facility is given in reference 17. The tunnel, shown schematically in figure 1 is an open-circuit, open-jet design. The inlet is provided with a high lengthto-diameter ratio honeycomb section and a series of turbulence suppression screens. These features, in conjunction with a large tunnel contraction, provide a spatially uniform, temporally steady flow with a controlled test section turbulence level of approximately 0.2 percent. Turbulence generators and grids can be inserted upstream of the nozzle to generate wake profiles (ref. 18) and a range of turbulence levels (ref. 19) in the test section.

The open jet test section is surrounded by a sealed anechoic chamber $4.9 \mathrm{~m}$ high, $5.5 \mathrm{~m}$ long (axial direction), and $6.7 \mathrm{~m}$ wide. Downstream of the test section the airflow enters a diffuser by way of a collector that has anechoic treatment on its flow impingement surface.

The diffuser operates unstalled and is thus not a major source of background noise. To avoid tunnel fan noise from propagating upstream into the anechoic chamber a Z-shaped muffling section with two right angle bends and parallel treated baffles is located between the diffuser and the fan. The $1100 \mathrm{~kW}$ centrifugal fan exhausts to the atmosphere through an exhaust tower.

Tunnel speed is determined from total pressure measurements at the contraction inlet and static pressure measurements within the sealed anechoic chamber. Since losses are confined to the boundary layer, total pressure upstream and downstream of the contraction are predicted and have been verified, to be equal. The test section velocity has been shown to be temporally steady.

Open Jet Geometry - A circular inlet nozzle with a radius, $R_{0}=0.455 \mathrm{~m}$, was employed in all tests. Initial facility tests reported in reference 17 identified an acoustic coupling between the inlet nozzle and the collector lip resulting in edge tones at high tunnel speeds. To suppress this noise mechanism, triangular tabs (see figure 2) were distributed around the nozzle periphery during the refraction angle correction experiment to disturb the azimuthal symmetry of the shear layer and prevent the generation of feedback tones. On the other hand, the shear layer thickness was increased by the tabs. Despite this alteration of shear layer thickness, the measured angle change associated with the wavefront propagation showed good agreement with the change calculated using the zero thickness shear layer theory of Amiet. 
After completion of the refraction angle correction study, a major anechoic chamber modification was performed. The facility changes included replacement of the acoustic treatment on the walls and on the surface of the downstream collector. These combined changes eliminated the nozzle lip to collector feedback problem permitting the nozzle to be used without tabs. The impact of the separate facility modifications on the feedback mechanism is not currently understood.

Operation of the open jet without tabs was advantageous to the refraction amplitude correction study which was conducted after the facility modification. The refraction amplitude correction experiment required measuring the acoustic field strength within the open jet potential core. To avoid measuring the source near-field characteristics it was necessary to maximize the separation distance between the source and the in-flow microphone. Without tabs the shear layer was thinner resulting in a larger distance between the jet centerline and the inner edge of the shear layer. The microphone could, therefore, be moved to a larger radius before it penetrated the turbulent shear layer velocity field.

Shear layer thickness in the presence or absence of the tabs is documented in the section titled Definition of the Open Jet Shear Layer. The importance of shear layer thickness on the refraction angle and amplitude correction experiments is discussed in the corresponding sections.

Anechoic Characteristics and Acoustic Source Selection - Figure 2 shows the interior of the anechoic chamber during the refraction angle correction experiments. The chamber walls, lined with $0.3 \mathrm{~m}$ fiberglass wedges, were found to be anechoic (ref. 17) for broadband noise over a $200 \mathrm{~Hz}$ to $20 \mathrm{kHz}$ range of calibration frequencies. In other words, the sound pressure level followed a $6 \mathrm{~dB}$ decay rate per doubling of source-to-observer separation distance. This decay rate was maintained within approximately $\pm_{0.25 \mathrm{~dB}}$ over the $200 \mathrm{~Hz}$ to $20 \mathrm{kHz}$ frequency range.

Since discrete tone source frequencies were used in the refraction angle correction experiment, it was necessary to conduct intensity versus distance calibration measurements to verify the free field behavior for a discrete tone. For these tests a speaker was located on the centerline of the open jet test section. A microphone was traversed radially outwards from the acoustic source at different angles to the jet axis. Figure 3 shows a typical result at $90^{\circ}$ to the jet centerline.

A close examination of the decay curves in figure 3 indicates

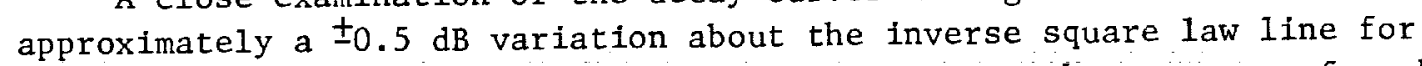
frequencies below $5 \mathrm{kHz}$. Such fluctuations suggest the presence of weak reflected waves in the anechoic chamber. These reflected waves combine with direct waves radiating from the acoustic source to the microphone resulting in nodes and antinodes distributed throughout the anechoic 
chamber. For example, a t0.5 dB variation represents a reflected-to-direct wave amplitude ratio of 0.06 if the two wavefronts are in phase. The refraction angle correction experiment could tolerate reflected waves of such amplitudes since the measurements emphasized phase difference and not absolute amplitude at the far field measurement station. This was verified analytically and is discussed in the section describing the angle correction experiment. Thus, the anechoic chamber was considered adequate for the discrete tone source signals_used in the angle correction experiment.

The source of the reflected waves was found to be the open jet face. This was determined while conducting pure tone free-field decay measurements prior to the refraction amplitude correction investigation. The purpose of these measurements was to check the acoustic characteristics of the anechoic chamber after it was lined with new fiberglass wedges.

The experimental set-up, with the new anechoic wedges is shown in the photo of figure 4. A suspended microphone traverse system was employed to facilitate changing the traverse line orientation relative to the open jet axis. The acoustic source used in the case was different from that used in the angle connection experiment. The previous source was replaced by a second unit which was smaller in size (fig. 4). Details of the source are given in the section titled Experimental Arrangement.

The new directional acoustic source was first pointed toward the downstream collector. The intensity versus distance plots showed numerous fluctuations about the inverse square-law curve with the microphone traversing at $90^{\circ}$ to the open jet axis. Figure 5 shows a typical trace for a $1.25 \mathrm{kHz}$ discrete tone. Both the inflow and far-field microphone stations used in the amplitude correction experiment are also shown so as to identify the spatial regions in which the free-field condition must be satisfied. Note that these positions correspond to $M=0$ operating conditions. The far-field microphone station changed slightly for non-zero Mach numbers.

With the source facing the microphone traversing at $90^{\circ}$ to the flow, the deviations from the inverse square-law line were reduced. It was concluded that when the highly directional source faced the downstream collector, the reflected sound wave amplitude arriving at the $90^{\circ}$ microphone was comparable to the direct source-to-microphone radiation. Thus the collector could alter the measured directivity pattern of discrete tone aeroacoustic noise mechanisms with a maximum directivity in the rear arc. This observation was, however, limited to low frequencies since the deviations from the Inverse square-law were much smaller at high frequencies.

It should be noted that the deviations in the intensity versus distance curves in figures 3 and 5 differ because the acoustic source locations are different. In addition, the acoustic treatment on the collector surface was changed between the refraction angle and amplitude correction experiments described by figures 3 and 5 . 
Since the refraction amplitude change was expected to be approximately zero at $90^{\circ}$ to the open jet, it was necessary to minimize any potential errors in the sound pressure level measurements. A criterion limiting the non-anechoic characteristics of the facility to $\pm 0.2 \mathrm{~dB}$ was, therefore, established. One of the methods considered for achieving this criterion used a random noise source to provide uncorrelated signals for the direct and reflected sound waves. The major disadvantage of this approach was the distribution of acoustic source output power over a large portion of the spectrum resulting in low acoustic energy levels per unit Hertz. These low levels would be masked by the microphone self-notse at the in-flow measurement station and the open jet background noise at the far-field station. In general, the signal-to-noise ratio could be expected to be low.

The above signal-to-noise problems were encountered by Ahuja et al in a shear layer refraction study reported in reference 15 . Their technique for overcoming the signal-to-noise limitation employed a cross-spectrum analysis which required two in-flow microphones and one far-field microphone. The measurement errors associated with this approach, however, precluded using the concept. In addition, the ability of the technique to recover the source signals was questionable at the highest Mach number of $M=0.4$ used in the present study. These limitations will be discussed further in the section titled Experimental Assessment of the Refraction Amplitude Correction Theory.

Due to the non-ideal anechoic characteristics obtained with the continuous discrete tone signal and the signal-to-noise limitation of the random notse signal, an alternate approach was developed. The method employed a discrete tone burst generated at the acoustic source. The burst duration time and repetition rate were selected to avoid interference between the direct radiated sound arriving at the microphone and the reflected sound from the collector. Using a discrete tone was also advantageous because it provided a large signal-to-noise ratio at both the in-flow and far field microphone stations. This was now possible because the total acoustic power was concentrated in the discrete tone signal rather than being distributed in a broadband random noise signal. The tone burst free-field decay measurement is compared with the continuous discrete tone measurement in figure 5 . The resulting radial traverse curve for the tone burst has few discernable deviations from the expected free field decay characteristics.

Measurements made over the complete angular range of $30^{\circ}$ to $140^{\circ}$ from the open jet axis showed similar free-field behavior. For example, figure 6 shows the intensity versus distance curve at $30^{\circ}$ from the open jet axis with the source facing the collector. Note that two separate traverses were needed to cover the source-to-far-field microphone distance. Figure 6 shows little evidence of interference between the direct radiated 
sound arriving at the microphone and the reflected sound from the collector surface. This was true for all source frequencies of $f=1.25,2.5,5$ and $10 \mathrm{kHz}$ used in the refraction amplitude correction experiment. Based on the success of the discrete tone burst technique, this acoustic source signal was selected for the refraction amplitude correction experiment. Additional calibrations demonstrating the accuracy of the method will be presented later.

It is probable that all open jet acoustic wind tunnel facilities with collectors suffer from the same non-anechoic environment for continuous discrete tones. Random noise calibrations have in the past masked this problem. The discrete tone provides a more severe test for evaluating freefield characteristics. Furthermore, by using a continuous traverse, in conjunction with the discrete tone the spatial distribution of nodes and anti-nodes can be used to identify the occurrence of wavefront interference. In comparison, measurements made at incremental distances from the acoustic source cannot be expected to coincide with the node and anti-anode locations. In this case, deviations from the free-field decay characteristics can be erroneously interpreted as scatter in the experimental data.

\section{Experimental Arrangement}

Refraction Angle Correction Experiment - Figures 2 and 7 show the anechoic chamber test arrangement employed for the angle correction measurements. The nozzle inlet and collector described earlier are evident in the photos.

An acoustic source, consisting of a speaker enclosed by an aerodynamic fairing (see fig. 7), was located in the open jet airstream. The fairing measurements were: $17 \mathrm{~cm}$ long (axial direction), $10 \mathrm{~cm}$ high, and $5 \mathrm{~cm}$ deep. Leading and trailing edges of the fairing were rounded. The wake shed by the body produced no significant discrete vortex shedding tones in the farfield acoustic spectrum.

The speaker fairing was held in place by a support arm which extended through the open jet to a rigid stand outside the flow. The support arm consisted of a short section of cylindrical tubing and an aerodynamically shaped fairing. Although the cylinder generated an aeolian tone, the dominant dipole radiation pattern was in a vertical direction perpendicular to the horizontal plane containing the speaker and the far-field microphones. Therefore this extraneous noise mechanism was not sensed. Similarly, noise generated by impingement of the highly turbulent shear layer on the aerodynamic fairing radiated In a vertical direction and was not sensed by the far-field microphones. Acoustic insulation was applied to the support stand and the exterior of the inlet nozzle. 
The speaker face, with its $2.5 \mathrm{~cm}$ diameter aperture, was covered with a plastic grid of the same aperture size. The grid prevented flow separation and distortion of the mean velocity field at the speaker-flow interface. The center of the grid aperture marked the acoustic source position. All far-field microphone locations were referenced to this station.

The acoustic wavefront angle outside the open jet was measured by a pair of far-field microphones. The microphones, designated as $\mathrm{m}_{1}$, and $\mathrm{m}_{2}$ in figures 7 and 8 , were mounted at grazing incidence on a rotating boom. The boom pivot point, located beneath the open jet, was aligned with the acoustic source position. Although figure 8 shows the source situated on the tunnel centerline, off-centerline stations were also investigated. The microphones scanned a horizontal plane at the same height as the tunnel centerline and the acoustic source. Different microphone separation distances, $\ell$, were used depending on the acoustic source frequency. The fixed microphone radius, $\mathrm{r}=1.83 \mathrm{~m}$, was measured to the midpoint of the microphone array. The radius was selected to permit scanning the boom close to the jet axis while still maintaining a large separation distance between the microphones and the downstream collector. This minimized the ratio of direct-to-reflected sound wave pressure amplitudes for acoustic rays propagating in the direction of the collector. The microphones were mounted rigidly using guy wires and braces to minimize any vibration or deflection when the open jet was operated.

The boom angular position was defined by the angle, $\theta_{\mathrm{m}}$, measured from the downstream jet axis. The angle was referenced to the midpoint between the two microphones. A line connecting the microphones formed approximately a $90^{\circ}$ angle with the radius, $r$. A potentiometer, attached to the boom pivot shaft, provided a voltage output corresponding to the boom angular position. The voltage was amplified and displayed on a digital voltage readout providing a resolution of $0.016 \mathrm{deg}$. The system was calibrated daily using the open jet centerline and a line perpendicular to the centerline as references. The accuracy of the calibration was approximately 0.3 degrees.

The intensity versus distance curves shown in figure 3 were obtained using the acoustic source described above. All refraction angle correction measurement were conducted at $\mathrm{r}=1.83 \mathrm{~m}$, where the free field behavior was well established.

Deviations from the inverse square law at distances less than $1 \mathrm{~m}$ were possibly due to the near-field characteristics. At small distances from the source, the aerodynamic fairing surrounding the speaker would appear as a baffle over which acoustic pressure fluctuations act. The microphone would then sense the geometric near field and possibly the acoustic near field of the distributed source region. Since the refraction amplitude correction and turbulence scattering experiments required in-flow microphone measurements at distances less than $1 \mathrm{~m}$ the above described source could not be used. Instead, a second source was developed for these studies. The characteristics of 
the source were described earlier in the subsection titled Anechoic Characteristics and Acoustic Source Selection. Additional details are given below.

Refraction Amplitude Correction Experiment - Figure 9 shows the anechoic chamber test arrangement employed in the amplitude correction experiment. Both in-flow and far-field traversing microphones are shown.

In-flow microphone measurements were required as part of this study, with the source-to-microphone separation distance being less than $0.455 \mathrm{~m}$. As noted earlier, deviations from the inverse square law decay curve occurred for separation distances less than $1 \mathrm{~m}$ when using the acoustic source developed for the angle correction experiment (fig. 3). For this reason a second acoustic source was developed.

The second source consisted of a commercially available 110 watt acoustic driver coupled to a tube using an inverse cone section. The $2.54 \mathrm{~cm}$ constant diameter tube terminated inside the open jet section. Locating the acoustic driver outside the test section with the tube crossing the open jet shear layer provided an additional advantage. The size of the acoustic driver was not limited thereby permitting a large and acoustically powerful driver to be used. This provided a significant improvement in signal-to-noise ratio compared to the acoustic source employed in the refraction angle correction equipment. In the latter case, the speaker size was limited by the aerodynamic fairing dimensions.

Microphone traverses in radial directions from the acoustic source were presented earlier in the discussion of the anechoic chamber characteristics. Measurements conducted at $90^{\circ}$ and $30^{\circ}$ from the downstream jet axis were shown in figures 5 and 6 . In addition to satisfying free-field conditions at the far-field microphone station, these figures demonstrate that free-field conditions are satisfied at the in-flow microphone station. Both figures indicate a free-field condition beyond $15 \mathrm{~cm}$ from the source. This ensured that the traverse line selected for the in-flow microphone was situated in the acoustic source free-field for all measurements.

The acoustic source orientation relative to the open jet centerline shows a $90^{\circ}$ bend at the tube termination in figure 4. This orientation was used only in the static free field decay measurements. The angle was changed to a $45^{\circ}$ orientation in the amplitude correction experiment to provide a maximum source directivity pattern in the downstream arc where the open-jet background noise was expected to be large. The final orientation is shown by the insert in figure 9.

Vortex shedding noise from the tube in figure 9 was suppressed by a splitter plate attached to the rear stagnation point. The objective was to suppress potential field fluctuations which the in-flow microphone would sense. While this suppression technique generated random noise, the radiation pattern was in a vertical direction perpendicular to the horizontal plane containing the source 
and the in-flow and far-field microphones. Therefore, this extraneous noise was not sensed. Similarly, noise generated by the impingement of the highly turbulent shear layer on the support tube radiated in a vertical direction and was not sensed by the microphones. Acoustic insulation was applied to the support stand and the exterior of the inlet nozzle.

The acoustic source signal was measured by the in-flow and far-field microphones designated as $m_{3}$ and $m_{4}$ in figure 10 . Microphone $m_{3}$ was traversed in straight line parallel to the intermittency line used to define the inner edge of the shear layer. A separation distance of $5 \mathrm{~cm}$ between the traverse Iine and the intermittency line avoided penetrating the turbulent shear layer with the in-flow microphone. Locating the in-flow microphone close to the edge of the shear layer maximized the source-tomicrophone separation distance thereby providing additional assurance of achieving a free-field condition at the microphone.

A nose cone was used for all in-flow microphone measurements. The microphone was always aligned parallel to the flow direction with the nose cone pointing upstream.

Far-field microphone, $m_{4}$, was traversed on a straight line parallel to the open jet at a sideline distance of $\mathrm{y}=1.83 \mathrm{~m}$. This distance was selected to maximize the refraction amplitude correction measurements which increase with sideline distance. The only limitation of this distance was the proximity of the anechoic chamber walls at angles close to the open jet axis. Like the in-flow microphone, a nose cone was used on the far field microphone with the microphone aligned parallel to the flow and pointing upstream. The reasons for using a nose cone outside the flow field are discussed in section titled Experimental Assessment of Refraction Amplitude Correction Theory.

The separate traverse units permitted locating the in-flow microphone at a specified radiation angle, $\theta_{c}$, with the far field microphone shifted to the refracted angle, $\theta_{\mathrm{m}}$, determined from refraction theory.

Although figure 10 shows the source situated on the tunnel centerline, off-centerline source positions were also investigated. As in the refraction angle correction experiment, the microphones scanned a horizontal plane at the same height as the tunnel centerline.

The in-flow and far-field microphone positions were measured from the open jet exit plane. Linear cable transducers were used to provide a voltage output corresponding to the microphone position. Voltage was amplified and displayed on a digital readout. The resolution of the linear position provided approximately a $0.5^{\circ}$ angular resolution at the in-flow microphone station and a $0.1^{\circ}$ resolution at the far-field microphone. 
The system was calibrated daily with the accuracy of the calibration at either microphone station being the same as the resolution capability.

Discrete Tone Scattering Experiment - The acoustic source and microphone arrangement were similar to the experimental set-up used in the refraction amplitude correction experiment, but a continuous discrete tone signal was used in place of the tone burst. Free-field decay measurements described earlier indicated a non-anechoic behavior of the chamber for continuous discrete tone sources. To avoid contamination of the far-field microphone signal by sound reflected from the collector, the sideline distance was decreased to $\mathrm{y}=0.91 \mathrm{~m}$ so that the direct radiation arriving at

the microphone dominated the reflected sound. It should be noted that this approach was not feasible in the refraction amplitude correction experiment because the amplitude change approaches zero as the sideline distance approaches the open jet radius. This is explained further in the section on theoretical formulation of the refraction problem.

Acoustic source near field effects were not expected to be a problem at the in-flow microphone station since the experiment employed the second acoustic source developed specifically to permit such measurements. Decay characteristics in figure 6 show few discernible deviations from the inverse square law line at the in-flow microphone station when using a discrete tone burst. Assuming the near field characteristics are the same for the continuous tone, the near field effects would be minimal. This is verified by the continuous tone decay curves in figure 5 which follow the inverse square law for distances less than $.4 \mathrm{~m}$.

\section{Instrumentation}

The various speakers and acoustic drivers used in the refraction and scattering experiments were powered by a 150 watt single channel amplifier. A sine wave generator with a tone burst output capability provided the amplifier input. The generator frequency was held to within $2 \mathrm{~Hz}$ of each source frequency evaluated in the test program.

Far field sound pressure levels were measured with commercially available $0.635 \mathrm{~cm}$ diameter condenser microphones. Protective grids were used in the angle correction experiment while nose cones were used in the amplitude correction and scattering study. Admittedly, the microphone frequency response was not flat due to the protective grid or nose, but comparisons of absolute sound pressure levels at different frequencies were not needed. For example, the angle correction experiment measured the acoustic wavefront propagation angle which was independent of radiated sound pressure level. The amplitude correction experiment used only the 
difference between the in-flow and far-field microphone sound pressure level at the same frequency. Since nose cones were used on both microphones, the frequency response characteristics were expected to be identical. Finally, the scattering experiment used the same sound pressure level measurement scheme as in the refraction amplitude correction experiment. As before, frequency dependent nose cone response characteristic were not needed. All microphones were calibrated daily with a $250 \mathrm{~Hz}$ pistonphone signal.

Microphone signals and the sine wave generator signal were recorded on magnetic tape during certain portions of the test. The frequency response of the FM tape system was flat over the frequency range investigated here.

Spectrum analysis during the angle and amplitude correction study was conducted with a narrow band, 500 line, real time spectrum analyzer - ensemble averager and a $1 / 3$ octave band analyzer. The $50 \mathrm{~Hz}$ effective bandwidth of the narrowband analyzer was found to be inadequate for the discrete tone scattering experiment. For this reason a second narrowband analyzer with a $0.25 \mathrm{~Hz}$ resolution was employed.

Cross-correlations and signal enhancement were performed using a 400 point, real time correlation and probability analyzer with the smallest available increment of resolution in the time domain being $0.2 \mu \mathrm{sec}$. Input signals were not filtered in the refraction angle correction experiment. Filtering was used in the amplitude correction experiment.

Mean velocity measurements of the open jet shear layer were acquired with a $0.025 \mathrm{~mm}$ diameter hot $\mathrm{film}$ operating in conjunction with a constant temperature anemometer system. The probe was calibrated in the tunnel test section.

\section{Test Program}

Refraction Angle Correction Measurements - Measurements were performed at various acoustic source locations, open jet Mach numbers, and source frequencies to assess these parameters in the refraction angle correction theory. Two coordinates defined the source location: the axial station, $X$, and the source-to-open jet-lip line separation distance, h. Both coordinates are shown in figure 8. Table I lists the test conditions evaluated in the present study with the source position coordinate normalized by the open jet radius. Included in the table is the ratio, $h / r$, which defines the far-field microphone position relative to the source-to-lip line distance. This parameter is an input to the experimental and theoretical angle and amplitude correction calculations. The lip line also defines 
the location of the zero thickness shear layer used in the refraction theory described in the section titled Theoretical Formulation of the Refraction Problem . Therefore, $h$ is at times referred to as the source-to-shear layer separation distance. It should be noted that since the source-tomicrophone distance, $r$, was fixed, changes in $h$ resulted in both $h / R_{0}$ and $h / r$ changing.

With the acoustic source situated at $x / R_{0}=1.33$ and $h / R_{0}=1$, the measurement angle, $\theta_{\mathrm{m}}$, was varied from $22.5^{\circ}$ to $105^{\circ}$ in $7.5^{\circ}$ increments. Angles less than $22.5^{\circ}$ were not investigated since microphone $\mathrm{m}_{2}$ in figure 8 would then penetrate the open jet shear layer. On the other hand, angles greater than $105^{\circ}$ were not investigated since an adequate separation distance between $\mathrm{m}_{1}$ and the upstream anechoic chamber wall must be maintained. Line-of-sight interference by the tunnel inlet between the source and microphone $\mathrm{m}_{2}$ did not occur until $\theta_{\mathrm{m}}$ exceeded $136^{\circ}$. Moving the source to $\mathrm{X} / \mathrm{R}_{\mathrm{O}}^{\prime}=2.66$ and 4.0 permitted increasing the upstream measurement angle to $127.5^{\circ}$. However, downstream measurement angles less than $30^{\circ}$ could not be attained due to shear layer spreading.

The same measurement angle range was used for the off-centerline source positions listed in Table $\mathrm{I}$. For $\mathrm{h} / \mathrm{r}=0.36$, measurement angles less than $30^{\circ}$ could not be investigated since $m_{2}$ penetrated the flow. As in the on-centerline test geometries, Iine-of-sight interference was not a problem.

Refraction Amplitude Correction Measurements - Measurements were performed using the same open jet Mach numbers and acoustic source frequencies investigated in the refraction angle correction experiment. Similar test conditions were investigated because the angle correction was needed to define the refracted ray path outside the open jet. The intersection of the refracted ray with the sideline determined the location of the far-field microphone in the amplitude correction experiment.

Far-field microphone measurements were conducted at a constant sideline distance instead of a constant radius location as in the angle correction experiment. The constant sideline geometry was selected because the refraction amplitude difference between the in-flow and far-field microphone was larger than in the constant radius geometry for angles close to the open jet axis. This point is illustrated by figure 44 and is discussed in the section titled Experimental Assessment of Refraction Amplitude Correction Theory. The larger amplitude change Improved the accuracy of the experiment relative to the $\pm 0.2 \mathrm{~dB}$ error band established from calibration measurements at static operating conditions. 
As shown in figure 10, angle $\theta_{c}$ inside the open jet determined the far-field microphone measurement angle $\theta_{\mathrm{m}}$. These angles are related by the refraction angle correction discussed later. Angle $\theta_{c}$ was selected as the principal variable permitting the in-flow microphone station to be held fixed as the open jet Mach number increased. This facilitated the measurement procedure. All comparisons between amplitude correction theory and experiment are, therefore, plotted as a function of $\theta_{c}$.

Test conditions for the amplitude correction experiment are listed in Table II. The parameter, $h / r$, which described the far-field microphone position in the angle correction experiment, has been replaced by the parameter, $h / y$. The theory predicts the amplitude change for an arbitrary value of $\mathrm{h} / \mathrm{y}$. Since the sideline distance, $\mathrm{y}$, was fixed, changes in $\mathrm{h}$ resulted in $h / R_{0}$ and $h / y$ changing.

With the acoustic source at $x / R_{0}=0.66$ and $h / R_{0}=1$, angle $\theta_{c}$ varied from $25^{\circ}$ to $90^{\circ}$. The same angular measurement range was used for the off-axis source position. Angles greater than $90^{\circ}$ were not investigated to avoid interference between the acoustic ray and the nozzle lip. Tests at a second downstream station, corresponding to $\mathrm{X} / \mathrm{R}_{\mathrm{O}}=2.66$, permitted making refraction amplitude correction measurements for the upstream directivity quadrant.

Discrete Tone Scattering Experiment - Measurements of the discrete tone scattering phenomenon were obtained at $\theta_{c}=90^{\circ}$ and several angles in the upstream and downstream quadrant. Tests were conducted over the same Mach number range as investigated in the refraction experiments. Two of the source frequencies corresponded to the $\mathrm{f}=5$ and $10 \mathrm{kHz}$ signals used in the refraction experiment. The motivation for selecting these frequencies was to verify the presence of discrete tone scattering observed in the refraction amplitude correction experiment. A third frequency of $\mathrm{f}=15 \mathrm{kHz}$ was expected to encounter strong turbulence scattering effects. The axial source positions covered the same measurement range as used in the refraction experiment. A listing of the test conditions is given in Table III. 
DEFINITION OF THE OPEN JET SHEAR LAYER

The objective of the mean velocity measurements described here was to document the shear layer characteristics of the UTRC Acoustic Research Tunnel as a function of axial position and Mach number. Measurements were obtained for the open jet operating with and without tabs attached to the periphery of the inlet nozzle. In the latter case, the velocity profile characteristics apply to the refraction amplitude and turbulence scattering experiments. Hot film anemometry was used for all measurements.

Radial distributions of the mean velocity will be presented first to illustrate how the shear layer features change with axial distance. Using these results, the shear layer growth rate will be presented as a function of axial location. Finally, to isolate changes in the velocity distribution with axial location, the velocity profiles are plotted in a dimensionless form using a similarity parameter based on the shear layer growth rate. The resulting curves from various axial stations collapse onto a single curve demonstrating similarity of the velocity profiles. This similarity, which has been well substantiated by other investigators, permits comparisons of the UTRC open jet test facility with other facilities.

In addition to mean velocity profile measurements, shear layer turbulence intensity was measured for the open jet operating without tabs. Measurements were obtained at each axial station to provide the necessary input to an existing theoretical scattering analysis.

\section{Mean Velocity Profiles}

Mean velocity profile measurements, with tabs attached to the nozzle periphery, were conducted at an azimuthal station coinciding with one of the tabs. Figure 11 shows the radial distribution of the mean axial velocity at various stations downstream of the nozzle exit for $M=0.2$ and 0.4 . Here the local velocity, $U$, is normalized by the potential core velocity, $U_{0}$. The axial position, $x$, and radial distance from the jet centerline, $R$, have been normalized by the jet radius, $R_{0}$. Although only two Mach numbers are presented, self-similar velocity profile shapes were obtained at all velocities when the axial station was held fixed.

The potential core and mixing zone are clearly identified in figure 11. The distributions exhibit a familiar behavior with the velocity profiles spreading radially with increasing axial distance. The bulge in the velocity profiles at $X / R_{0}=0.66$ was due to the severe mixing forced by the tabs 
attached to the open jet nozzle. This feature is smoothed out with increasing distance from the nozzle exit disappearing altogether beyond $X / R_{0}=1.33$.

Figure 12 shows the normalized velocity profiles without tabs attached to the inlet nozzle. The bulge observed at $x / R_{0}=0.66$ in figure 11 is now absent. Here the open jet shear layer grows naturally.

\section{Shear Layer Thickness}

Figure 13 shows a schematic representation of the coordinates needed to describe the shear layer thickness. Since there is no universally accepted definition of shear layer boundaries, the inner and outer edges of the shear layer are defined here by the 90 percent $\left(U / U_{O}=0.9\right)$ and 10 percent $\left(U / U_{0}=0.1\right)$ velocity lines, respectively. The distance from the jet centerline to the half-velocity 1 ine $\left(U / U_{O}=0.5\right.$ ) is denoted by $R_{I / 2}$. The local shear layer thickness, $\delta$, is defined as the difference between the inner and outer edges of the open jet.

The average radial position of the inner and outer edge of the shear layer over a range of Mach numbers was determined from the velocity profiles in figures 11 and 12 . The results are shown in figures 14 and 15 as a function of normalized radius. Also shown is the average half-velocity position. The shear layer thickness was calculated as a function of axial position using these average characteristics. The results, shown in figure 16, describe the shear layer growth rate.

The analytical solution derived by Goetler (see ref. 20, page 96) describing the growth of a two dimensional shear layer is also shown in figure 16. The analysis was used to calculate the inner and outer edges of the shear layer (without tabs present) defined by the 90 percent and 10 percent velocity points. Here the Goetler solution is considered adequate for determining the growth rate of an axisymmetric jet flow within the first two jet diameters since the flow is nearly two-dimensional in the region. Good agreement between theory and axisymmetric jet flows is also evident from the measurement of Candell (ref. 21) which are shown in figure 16. Note that tabs were not used in reference 21 .

Several conclusions can now be drawn regarding the UTRC open jet test section. First, with tabs attached to the inlet nozzle, the open jet shear layer is larger than an axisymmetric jet without tabs. This is due to the early mixing forced by the presence of the tabs. However, at large $X / R_{O}$, the shear layer thickness approaches the standard jet result. Finally the initial shear layer growth rate, with the tabs removed, can be predicted by the Goetler analysis for a two dimensional plane jet. 
Tabs were attached to the nozzle periphery during the refraction angle correction measurements. The resultant thickening of the shear layer provided a more severe test for verification of the vortex sheet shear layer model used in the refraction theory. Despite the thickening of the shear layer by the tabs, the refraction angle changes showed good agreement with theory.

\section{Similarity of Mean Velocity Profiles}

To permit direct comparison of the open jet velocity profiles at various axial stations, the mean velocity distributions in figures 11 and 12 were plotted versus the similarity parameter, $\eta$. Here $\eta$ is defined as, $\eta=\left(R-R_{1 / 2}\right) / \delta$. A comparison of the normalized velocity profiles is given in figures 17 and 18 for three different axial stations and two Mach numbers, $M=0.2$ and 0.4 . The various curves at the three stations collapse onto a single curve.

Also shown in figures 17 and 18 is the standard hyperbolic tangent analytic curve used for two-dimensional plane-jet shear layers. The good agreement between experimental data and the hyperbolic tangent curve means this relationship adequately describes the UTRC shear layer characteristics.

Turbulence Intensity

Shear layer turbulence intensity profile measurements were conducted for the open jet with the tabs removed. Radial distributions were obtained at the same axial stations for which mean velocity profiles were measured. Figure 19 shows the $M=0.4$ turbulence intensity profiles plotted as a function of the similarity parameter, $n$.

The results indicate that the similarity parameter used for the mean velocity profiles also collapse the different turbulence intensity profiles onto a single curve. Peak turbulence intensity is approximately constant between the upstream station and the farthest downstream station.

To assess the effect of changing Mach number, radial distributions are compared at $X / R_{0}=4$ for two different Mach numbers. The results, shown in figure 20, verify that the turbulence intensity profiles are independent of open $j$ et velocity. 
The turbulence intermittency boundary used to define the inner edge of the shear layer is shown in figure 15. A knowledge of this boundary was necessary to avoid locating the in-flow traversing microphone in the turbulent flow. This boundary was determined by monitoring, on an oscilloscope, the fluctuating voltage signal from the hot film probe and noting when the random fluctuations disappeared. The advantage of this technique is that it is sensitive to the occasional turbulent bursts which occur near the edge of a shear layer. 
THEORETICAL FORMULATION OF THE REFRACTION PROBLEM

Review

The basic approach for most of the present shear layer correction theories was initially given by Amiet (ref. 7). This analysis treated the case of a source beneath a plane zero-thickness shear layer. The observer was assumed to be in the geometric and acoustic far field of the source. However, the shear layer could be at any distance from the source. There were no requirements for the source to be acoustically compact or a point source. This analysis, based on the previous work of Ribner (ref. 2) and Miles (ref. 3), requires that the measurements made outside the shear layer be corrected in both angle and amplitude. The result is a correction method which is independent of frequency and source type (i.e., monopole, dipole, quadrupole).

Independence of frequency and source type and the absence of any requirements for the source to be compact or the shear layer to be in the far field of the source is rigorously true only for the plane shear layer case. These points were not proved in the original derivation of Amiet (ref. 7), however, the rigorous derivation of Amiet (ref. 10, Appendix 1) for the plane shear layer verified these statements. This has led to confusion on the part of some readers who continue to base their conclusions on the original derivation. The only requirement for the second derivation is that the observer distance be large compared to the source dimensions and the acoustic wavelength.

The analysis for the single plane shear layer is simple because there are no multiple reflections. The more realistic case of a cylindrical shear layer does require additional assumptions to be made. Jacques (ref. 8) first treated the cylindrical shear layer case for a source on the jet centerline. The multiple reflections which occur for this case are neglected since the reflection coefficient is generally small at tunnel Mach numbers of interest. Also, the acoustic wavelength must be small compared to the source-shear layer distance. This restriction is easily satisfied since figure 4 of reference 10 indicates a wavelength smaller than a few jet diameters is generally small enough. For open jet diameters encountered in practice, this is not usually a severe limitation. The cutoff frequency (f requency below which the surrounding chamber becomes non-anechoic) of open jet acoustic test facilities typically correspond to a wavelength to diameter ratio on the order of 1 .

The high frequency case of a source on the centerline of a cylindrical shear layer with a thickness significantly greater than a wavelength was treated by Tester and Morfey (ref. 9). Although not explicitly derived as a correction for open-jet wind tunnel measurements, the results of the analysis were applied toward this end by Ahuja et al., in reference 15. 
It is interesting to note that the shear layer corrections for the two cases of zero thickness (as formulated by Amiet) and infinite thickness shear layers (as formulated by Ahuja et al) differ by only a few hundredths of a decibel over most of the angular range as shown in reference 10 . This would be expected since the basic difference between the two corrections is that the zero thickness case assumes a reflected wave exists while the infinite thickness case assumes that all the energy is transmitted. Since the amplitude of the reflected wave is small over most of the angular range (see e.g., reference 7) and since the reflected energy is proportional to the square of the amplitude, one should expect little difference between the corrections. At the extreme angles where the reflected wave can have a significant magnitude, both the zero thickness and the infinite thickness corrections should be used with caution since neither correction accounts for multiple reflections within the shear layer. Thus, the correction procedure for an infinite thickness shear layer used by Ahuja et al (ref. 15) has the same angle correction as that of Amiet (ref. 7 and 10) and Jacques (ref. 8) and an amplitude correction differing by a few hundredths of a dB over most of the angular range. It is, for all practical purposes, equivalent to the correction procedure for a zero thickness shear layer. A more detailed comparison between the various zero thickness, infinite thickness, plane shear layer and cylindrical shear layer results is given in reference 10.

\section{Shear Layer Correction for An Off-Axis Acoustic Source}

For the case of a source on the centerline of a cylindrical shear layer, as was discussed in reference 10 , the shear layer must be in the acoustic far field of the source for the shear layer correction to be independent of source type and frequency. Thus, ray acoustics principles are applicable and the shear layer correction can be derived by ray tracing arguments. For the case of a source off the centerline, the derivation will also be based on ray acoustics. The same far-field assumption required for the on-axis source solution applies here. The main difference between this case and the oncenterline case is that the algebra for the ray tracing of the off-center case becomes significantly more complex. The basic principles governing the crossing of the shear layer by a ray remain the same, however.

Figures 21 and 22 show the basic geometry and the coordinate system used in the derivation. The zero thickness cylindrical shear layer has a radius, a, and the source is located off the centerline by a distance $a-h$. A Cartesian coordinate system has the origin located at the source, $S$, the $x$ axis parallel to the tunnel axis and the $z$ axis along the normal from the tunnel centerline to the source. A ray travels from the source along the 
path labeled $r_{1}$ crossing the shear layer at point $x_{1}, y_{1}{ }^{\prime}, z_{1}{ }^{\prime}$, and continues along path $\mathrm{r}_{2}$ to the observer 0 at $\mathrm{x}_{2}, \mathrm{y}_{2}{ }^{\prime}, \mathrm{z}_{2}{ }^{\prime}$. The plane tangent to the shear layer at point $x_{1}, y_{1}^{\prime}, z_{1}^{\prime}$ makes an angle $\alpha$ with the $x-y$ ' plane. Angles $\theta_{c}$ and $\phi_{c}$ describe the ray path beneath the shear layer, $\theta_{t}$ and $\phi_{t}$ describe the ray path above the shear layer and $\theta_{m}$ and $\phi_{m}$ describe the observer position relative to the source.

The angle $\theta$ is the angle between a given line and the $x$ axis or a line parallel to the $x$ axis. Thus $\theta_{c}$ is the angle between the ray beneath the shear layer and the $x$ axis, $\theta_{t}$ is the angle between the transmitted ray and a line parallel to the $x$ axis at the shear layer crossing point, and $\theta_{m}$ is the angle between the $x$ axis and the line joining source and observer. The angle $\phi$ is the angle between the $x-y$ plane and a plane containing a given line and parallel to the $x$ axis. Thus, $\phi_{c}$ is the angle between the $x-y$ plane and the plane defined by the $x$ axis and the ray beneath the shear layer, $\phi_{t}$ is the angle between the $\mathrm{x}-\mathrm{y}$ plane and the plane containing the transmitted ray and parallel to the $x$ axis, and $\phi_{m}$ is the angle between the $x-y$ plane and the plane containing the $\mathrm{x}$ axis and the observer point. The unprimed coordinate system is defined by rotating the $x, y^{\prime}, z^{\prime}$ coordinate system through an angle $\alpha$ about the $x$ axis so that $z$ is normal to the tangent plane.

The detailed derivation of the off-axis source correction is given in Appendix A. Results of this calculation can be sumarized as follows: the amplitude correction is different from the on-axis case for all sourceobserver positions. In the special case of an observer located in the plane containing the source and open-jet centerline, the angle correction is identical to that obtained for an on-axis source or for the case of a plane shear layer.

A listing of the generalized computer program which predicts the refraction effects described above is given in Appendix A. A sample printout is also provided. The program is extremely short thereby simplifying the task of generating refraction amplitude correction predictions.

\section{Theoretical Refraction Predictions For Comparison With Experiment}

The primary objective of the experimental effect was to measure the refraction angle and amplitude changes associated with acoustic wave-front propagation through an open jet shear layer. While various combinations of source-observer locations could be evaluated the present study was limited to acoustic source and far-field microphone positions situated in a plane which coincided with the jet axis. Under these conditions, angles $\phi_{c}=90^{\circ}$ 
and $\alpha=0^{\circ}$ in figures 21 and 22. The resulting propagation path, SBO in figure 22, was thus confined to a plane normal to the tangent plane at the shear layer crossing point.

The three-dimensional geometry in figures 21 and 22 can therefore be replaced by the two-dimensional geometry in figure 23 . Here wave fronts propagating in the direction, $\theta_{c}$, inside the afrstream propagate in the direction, $\theta_{t}$, outside the open jet. The actual path of an acoustic ray is described by the points SBO. The change from $\theta_{c}$ to $\theta_{t}$ is a result of refraction by the shear layer. Without flow the sound propagates at angles $\theta_{\mathrm{m}} \cdot$

The refraction angle and amplitude corrections convert measurements at the observer station ( 0 ) in figure 23 to either the constant radius station (C) or constant sideline station (D) on the non-refracted path. Selection of the particular conversion depends on the far-field microphone array geometry used to measure the acoustic source directivity pattern. Whichever microphone geometry is used, the corrected sound pressure level represents an equivalent measurement in the absence of the shear layer. In other words, the shear layer is considered located at infinity with uniform flow existing between the source and the far-field microphone at either station $C$ or $D$.

Several features of the refraction theory are worth noting. First, the transmitted angle, $\theta_{t}$, is the same whether a constant radius or constant sideline measurement is selected. The amplitude correction is partially a function of the difference in path length between the source and the far-field microphone on the refracted and non-refracted acoustic ray. The amplitude correction is also a function of the difference in ray divergence on the convected ray path inside the open jet (SB) and on the refracted ray path (BO) outside the open jet. Once the amplitude correction is calculated for either station $C$ or $D$ on the non-refracted ray, the amplitude at the remaining station is simply calculated using the inverse square-law. For example, if $\mathrm{dB}_{C}$ represents the decibel amplitude correction needed to convert the measurement at station 0 to station $C$, then the decibel correction for station $D$ is $\mathrm{dB}_{\mathrm{D}}=\mathrm{dB}_{\mathrm{C}}-10 \log (\mathrm{SD} / \mathrm{SC})^{2}$.

If the sideline microphone distance approaches the open jet radius the amplitude correction decreases to a finite value independent of the open jet radius. This finite value represents the difference in acoustic energy between the incident and reflected waves inside the open jet. It should be noted that the effect of path length difference between the refracted and nonrefracted ray paths does not influence this conclusion. This is because the path difference approaches zero as the sideline microphone approaches the open jet radius. Furthermore, for measurement locations just outside the open jet the ray tube has diverged only a small amount compared to the ray tube inside the open jet. Thus, changes in the divergence rate across 
the shear layer would not be perceptible for out-of-flow microphone stations close to the open jet. Amplitude changes across the shear layer would insensitive to divergence rate changes. Consequently, the amplitude change for microphone stations close to the open jet radius is limited to the difference between the acoustic energy of the incident and reflected waves inside the open jet. This amplitude change is represented by equation A.21 in reference 7 .

Figure 24 shows the predicted refraction angle correction for a constant radius microphone geometry as a function of open jet Mach number. Specific coordinates for the source and far-field microphone positions are given by the parameters $h / R_{0}$ and $h / r$. The values for these parameters correspond to the conditions tested in the experimental study and listed in Table 1 . The significance of the angle correction is evident when comparing the difference between angles $\theta_{\mathrm{m}}$ and $\theta_{\mathrm{c}}$. For example at $\mathrm{M}=0.4$ a microphone measurement at $\theta_{m}=60^{\circ}$ corresponds ${ }^{c}$ to a measurement on an acoustic ray initially propagating at $\theta_{c}=45^{\circ}$ inside the open jet.

Moving the source of $f$ axis results in a significant angle change as shown by figure 25 for the fixed Mach number, $M=0.3$. The range of off-axis source positions is defined by either the parameter $h / R_{0}$ or the parameter $g$. The latter variable is defined as $g=1-\frac{h}{\bar{R}}$ where $h$ is measured from the shear layer closest to the observer. The silall insert in figure 25 defines the range of the parameter $g$.

Refraction amplitude correction predictions are shown in figure 26 for two Mach numbers with the source situated on the open jet axis. The amplitude correction is added to the sound pressure level measured at angle $\theta_{\mathrm{m}}$ to give the equivalent noise level obtained on the non-refracted ray identified by angle $\theta_{c}$. The curves indicate a small negative amplitude correction for measurement angles close to the open jet axis, and a positive correction in the upstream quadrant. The effect of moving the source off-axis is significant as shown in figure 27 for a fixed Mach number, $M=0.3$.

Figures 28 through 31 show a similar set of curves for the constant sideline refraction angle and amplitude corrections. Predictions are presented for both on-axis and off-axis acoustic source positions. Here the sideline parameter, $\mathrm{h} / \mathrm{y}=0.25$, was selected equal to the constant radius parameter, $h / r=0.25$, used in figures 24 through 27 . A comparison of the amplitude corrections at the same angle $\theta_{\mathrm{m}}$ in figures 26 and $30 \mathrm{a}$ shows a significant difference between the two geometries. Figure $30 \mathrm{~b}$ shows the same amplitude correction plotted versus angle $\theta_{C}$. All figures presented in the section describing the experimental assessment of the amplitude correction are plotted as a function of $\theta_{c}$. 
It is important to recognize that the theoretical predictions in figures 24 - 31 are a function of the source position $\left(h / R_{0}\right)$ and the microphone position $(\mathrm{h} / \mathrm{r}$ or $\mathrm{h} / \mathrm{y})$. The predicted angle and amplitude correction curves are replaced by a different set of curves if these parameters change. 


\title{
EXPERIMENTAL ASSESSMENT OF REFRACTION ANGLE CORRECTION THEORY
}

\author{
Experimental Approach
}

The experimental refraction angle correction for the geometry in figure 23 was obtained using the test set up shown in figure 8. Acoustic ray angle changes were determined from measurements of the constant phase or wave-front surfaces outside the airstream. In this regton, the acoustic ray is perpendicular to the wave-front. Once the wave-front angle at the observer location is known, the ray normal to the wave-front ray can be traced back to the point, $\mathrm{x}_{0}$, at which the sound emerged from the shear layer. The original propagation angle inside the open jet is then obtained from the relationship $\theta_{\mathrm{c}}=\arctan \left(\mathrm{h} / \mathrm{x}_{\mathrm{o}}\right)$.

The wave-front angle was measured in the following manner. Sound was generated at discrete frequencies by an acoustic source inside the open jet. The phase difference, between the signals arriving at microphones $m_{1}$ and $m_{2}$ in figure 8 , was then determined by cross-correlating the signals. From this, the wave-front angle relative to the microphone array was calculated permitting the ray to be traced back to the shear layer crossing point.

\section{Measured Phase Difference and Calculated Angle Change}

It now remains to derive the equations linking the measured phase difference and the original propagation angle, $\theta_{c}$, inside the free jet. The modeling problem is shown in figure 32. For $M=0$, the angle between the wave front arriving at $\mathrm{m}_{2}$ and the line connecting the array is denoted by $\mu_{o}$. This parameter accounts for small differences in the distance, $\Delta \mathrm{r}$, (fig. 32) between the source and the individual microphones. This difference is due to inaccuracies in placing the two microphones at exactly the same radial distance from the source. The difference was consistently held to less than $0.25 \mathrm{~cm}$ during the experiment. Assuming this value of $\Delta \mathrm{r}$ results in a maximum value of $\mu_{o}=0.016$ radians for the smallest microphone separation distance, $\ell$, shown in figure 32 .

If the askew microphone array orientation were neglected, errors would arise in the phase shift measurement. For example, if $\Delta \mathrm{r}=0.25 \mathrm{~cm}$, then at $10 \mathrm{kHz}$ a 0.07 cycle phase shift occurs between $m_{1}$ and $m_{2}$. Since the present experiment required an accuracy of 0.02 cycles it was necessary to account for the skewed microphone alignment. That is, the measured phase shift at $M=0$ was subtracted from the phase shift obtained at finite Mach number. This effectively calibrated the measurement of phase shift to account for microphone placement error. The mathematical details are presented below.

For $M=0$, the total phase difference (expressed in cycles) between constant phase points arriving at $\mathrm{m}_{1}$ and $\mathrm{m}_{2}$ is given by the relation 


$$
\Delta_{T}=\Delta+\left(\Delta_{1}-\Delta_{2}\right)
$$

where

$$
\Delta=\frac{f \ell}{c_{0}} \sin \mu_{0}
$$

The parameters, $\Delta_{1}$ and $\Delta_{2}$, account for the phase lag between the acoustic input signal to each microphone diaphragm and the final voltage signal applied at the correlator. Nominally, at $M=0$ this phase lag is less than 0.1 cycles for the frequency range employed in the present angle correction experiment. The microphone cartridge phase lag characteristics are included in this parameter.

The parameter, $\Delta_{\mathrm{T}}$, was measured directly by cross-correlating the periodic signals arriving at the adjacent microphones. Figure 33 illustrates the correlator time delay trace. Based on the phase shift of the first maximum, $\Delta_{\mathrm{T}}=\tau \cdot \mathrm{f}$. The magnitude of $\tau$ has been exaggerated to make it perceptible in the figure. Nominally, $\Delta_{T}$ is less than 0.1 cycles at $M=0$. The location of the first maximum in the correlation function was defined as the mid-point between the zero cross-over points. In figure 33 the mid-point of the second cycle and a knowledge of the period were used to locate the midpoint of the first cycle. By using two cross-over points irregularities in the correlation function were averaged. The term "irregularities" is used here to describe a deviation of the measured correlation functions from a smooth sinusoidal behavior. These deviations occurred when both microphones sensed open jet shear layer background noise in addition to the source noise at non-zero Mach numbers.

For $M \neq 0$, the total phase difference is given by

$$
\Delta_{T}^{\prime}=\Delta^{\prime}+\left(\Delta_{1}-\Delta_{2}\right)
$$

where

$$
\Delta^{\prime}=\frac{f l}{c_{0}} \sin \left(\mu_{0}+\mu_{1}\right)
$$

Here $\mu_{1}$ describes the angle between the two wavefronts in figure 32. Solving for the difference $\Delta_{T}^{\prime}-\Delta_{T}$ gives

$$
\Delta_{T}^{\prime}-\Delta_{T}=\Delta^{\prime}-\Delta=\frac{f l}{C_{0}}\left[\sin \left(\mu_{0}+\mu_{1}\right)-\sin \mu_{0}\right]
$$

Appendix $B$ shows how this expression may be simplified for the small values of $\mu_{0}<0.016$ radians used in the present experiment. Solving for $\mu_{1}$ gives 


$$
\mu_{1}=\sin ^{-1}\left[\frac{c_{0}}{f \ell}\left(\Delta_{T}^{\prime}-\Delta_{T}\right)\right]
$$

From this $\theta_{c}$ can be determined using the relationship

$$
\theta_{c}=\tan ^{-1}\left(n / x_{0}\right)
$$

where

$$
x_{0}=r \cos \theta_{m}-\left(r \sin \theta_{m}-n\right) \cot \left(\mu_{1}+\theta_{m}\right)
$$

The expression for $\mathrm{x}_{\mathrm{o}}$ is derived in Appendix $\mathrm{C}$.

Accuracy requirements of the phase measurements necessitated small time delay increments in the cross-correlation function calculation. Depending on the acoustic source frequency, the following time delay resolution values, $\mathrm{d} \tau$, were used: a) $\mathrm{f}=10 \mathrm{kHz}, \mathrm{d} \tau=1 \mu \mathrm{sec}, \mathrm{b}) \mathrm{f}=5 \mathrm{kHz}$, $\mathrm{d} \tau=2 \mu \mathrm{sec}, \mathrm{c}) \mathrm{f}=2.5 \mathrm{kHz}, \mathrm{d} \tau=5 \mu \mathrm{sec}$, and d) $\mathrm{f}=1.25 \mathrm{kHz}, \mathrm{d} \tau=10 \mu \mathrm{sec}$.

Background Noise and Non-Anecholc Effects

The above cross-correlation approach was successful only at $M \leq 0.1$ For higher open jet Mach numbers, the facility random background noise dominated the cross-correlation function as is illustrated by the two-microphone correlation function in figure 34. Although a periodic waveform is present in the cross-correlation function, the measured result is not suitable for solving equation 6 for the wavefront propagation angle. To verify that the time delay trace is dominated by facility noise, a second trace is shown with the acoustic source turned off.

The background noise dominated the cross-correlation function despite the $12 \mathrm{~dB}$ signal to noise ratio shown in figure 35 . This occurred because the microphone input signals to the correlator were not filtered permitting the complete background noise signal to contribute to the cross-correlation. It may appear that this problem can be circumvented by narrowband filtering the microphone spectrum shown in figure 35 to pass only the pure-tone component. However, this still retains in the correlation function a contribution from the cross-correlation of the filtered narrowband microphone signals. The periodicity of this contribution corresponds to the filter cutoff frequencies. Since these frequencies are close to the acoustic source frequency, a portion of the narrowband random contribution would remain in the cross-correlation. 
An alternate cross-correlation technique developed by Schlinker (ref. 23) was therefore employed. The method involves cross-correlating the individual microphone signals with the periodic input signal to the acoustic driver system. The random signals due to facility noise are rejected leaving only the periodic component. The success of the method is illustrated by the correlation traces in figure 36 . Here, $S_{1, s}$ and $S_{2, s}$ represent the individual microphone-signal generator cross-correlation functions. To verify that the traces are independent of the facility noise, one trace is shown with the source turned of $f$.

The mathematical details linking the correlations in figure 36 with the microphone orientation angle $\mu_{1}+\mu_{0}$ are presented in Appendix D. The final result for the phase difference at non-zero Mach number is

$$
\Delta_{T}^{\prime}-\Delta_{T}=\eta_{1}-\eta_{2}-\Delta_{T}
$$

where $n_{1}, n_{2}$ represent the phase difference between the acoustic driver signal and the individual microphone signals. Equation (9), when used in conjunction with equation (6), gives (for small $\mu_{0}$ ),

$$
\mu_{1}=\sin ^{-1}\left[\frac{c o}{f l}\left(\eta_{1}-\eta_{2}-\Delta_{T}\right)\right]
$$

A brief discussion of the measured phase difference is now warranted since the angle correction is derived from this parameter. Figure 37 shows the experimentally determined phase difference, $\Delta_{\mathrm{T}}{ }^{\prime}-\Delta_{\mathrm{T}}$, for a $2.5 \mathrm{kHz}$ acoustic source frequency with the open jet nperating at $M=0.3$. The source position corresponded to Test Configuration 1 in Table $I$. Two different microphone spacings were used in the measurement. For angles near $90^{\circ}$, where refraction is minimal, a large separation $(\ell=0.48 \mathrm{~m}$ ) was used to obtain a measurable phase shift. In contrast, at angles close to the jet axis where the phase shift is large a smaller separation $(l=0.3 \mathrm{~m})$ was used.

The measured data in figure 37 are compared to the phase difference calculated from the refraction theory described in Appendix A. The figure shows excellent agreement between the theory and experiment over the complete range of measurement angle. The measured phase was then used to calculate the angle correction associated with the transmission of sound through the shear layer. 
The above described experimental approach assumes that the wave front arriving at the far-field microphone array is plane. The possible error in this assumption was evaluated by calculating the time for the sound to travel from the source to the individual far-field microphones. The difference between these times was then compared to the time calculated using the plane wave assumption.

For a point source in a flow, the phase, $\theta$, of the waves is

$$
\theta=\left\{t+\frac{1}{C_{0} B^{2}}\left[M x-\sqrt{x^{2}+\beta^{2}\left(y^{2}+z^{2}\right)}\right]\right\} \omega
$$
If $\boldsymbol{\theta}=0$ at $t=0$, then the same wave front will reach the point $\left(x_{0}, 0, h\right)$
at time $t_{0}$ where

$$
C_{0} \beta^{2} t_{0}+M x_{0}=\sqrt{x_{0}^{2}+\beta^{2} n^{2}}
$$

Here $\mathrm{X}_{\mathrm{o}}$ is the shear layer crossover in figure 23. Using $\tan \theta_{\mathrm{c}}=\mathrm{h} / \mathrm{x}_{\mathrm{o}}$ along with equation A.18 relating $\theta_{c}$ and $\theta_{c}$ gives

$$
t_{0}=\frac{n\left(1-M \cos \theta_{1}\right)}{c_{0} \zeta_{1}}
$$

The time for the wave to travel from $x_{0}$ to the observer at 0 (see figure 23) is

$$
i_{1}=\frac{r \sin \theta_{m}-h}{c_{0} \sin \theta_{1}}
$$

The total time for a sound wave to travel from the source to a far-field microphone is then $t_{2}=t_{0}+t_{1}$. The exact time difference, $\Delta t_{e}$, for the sound to reach the two separate microphones is obtained from the difference between the value of $t_{2}$ calculated for each microphone.

On the other hand, the approximate calculation treating the wave front as plane gives for the time difference (see figure 32).

$$
\Delta t_{0}=\frac{l}{c_{0}} \sin \left(\theta_{1}-\theta_{m}\right)=\frac{l}{c_{0}} \sin \mu_{1}
$$

This expression is easily derived from equation (B.5). Note that for this calculation the angles $\theta_{t}$ and $\theta_{\mathrm{m}}$ are averages calculated for an observer midway between the two microphones. 
Figure 38 shows the percent error between the exact $\left(\Delta t_{e}\right)$ and approximate $\left(\Delta t_{a}\right)$ solutions defined as

$$
\text { PERCENT ERROR }=100 \frac{\Delta t_{e}-\Delta t_{0}}{\Delta t_{e}}
$$

The calculations are for the maximum microphone separation used which was $\ell=0.48 \mathrm{~m}$. The error decreases as the square of the microphone separation so that the next smaller separation value listed in figure 32 would give an error approximately $1 / 4$ as large. Even at the large separation, however, the error was very small, on the order of a few percent. This justifies the experimental approach described earlier.

The above error estimate assumes that the acoustic wave front arriving at the far-field microphone array originates at the sound source. Measurements of the free-field decay characteristics, discussed earlier in the description of the experiment, indicated the presence of waves reflected from the downstream collector surface. For the case of a continuous discrete tone acoustic source signal these reflected waves combined with the direct acoustic waves arriving at the microphone. This resulted in nodes and antinodes distributed throughout the chamber. Deviations from a free-field anechoic environment were only $\pm 0.5 \mathrm{~dB}$ which represented a reflected-todirect wave amplitude ratio of 0.06 if the two wavefronts were either in phase or $180^{\circ}$ out of phase.

The refraction angle correction experiment could tolerate reflected waves of such amplitudes since the measurements emphasized phase difference and not absolute sound pressure level measurements at the far-field microphone measurement station. This was verified analytically in Appendix E. . The estimated error in the phase measurement was found to be 0.0091 cycles. This error is comparable to the experimental accuracy of the phase measurement and is less than the 0.02 cycle accuracy required in the experiment.

It is worthwhile to perform an error analysis to determine the sensitivity of the corrected angle, ${ }^{{ }_{c}}$, to errors in the experimentally determined phase difference in the argument of equation 10 . Such an error analysis is provided in Appendix $F$ for the case of $\theta_{m}=90$. Here the magnitude of the phase difference is a minimum (see figure 37 ) so that small errors in the measured phase difference become significant. Considering the case of $M=0.4$, the sensitivity of $\theta_{c}$ to changes in the phase difference is estimated by the differential equation $\mathrm{d}_{\mathrm{c}}=1.6 \mathrm{~d}\left(\Delta^{*}\right)$ where $\Delta^{*}=n_{1}-n_{2}-\Delta_{\mathrm{T}}$. Assuming a 0.02 cycle error in the measurement of $\Delta^{*}$ the error in $\theta_{c}$ is calculated to be $1.8^{\circ}$. This is considered to be small compared to the absolute magnitude of $\theta_{c}=72^{\circ}$ in this case. Thus, the accuracy of the phase measurement had a negligible effect on the experimentally determined value of $\theta_{c}$. 
Comparison of theory and experiment is given first for the case of an on-axis source location. Shown in figure 39 is a comparison between the measured and theoretical angle correction as a function of open jet Mach number and acoustic source frequency. The source and far-field microphone positions correspond to Test Configuration 1 . Specific coordinates for this geometry are given in figure 39(a). Only two curves are shown in each plot to avoid crowding the experimental data points.

The agreement between theory and experiment is considered good particularly at frequencies above $1 \mathrm{kHz}$ where the phase differences are large and measuremient accuracy is correspondingly good. At $1 \mathrm{kHz}$, the small measured phase difference introduced some scatter into the experimental data points. To obtain a quantitative assessment of the refraction correction theory, the absolute difference between the theoretical curves and the data can be calculated. Considering the measurement angle, $\theta_{\mathrm{m}}=37.5^{\circ}$, where the correction is significant, the average difference was $1.25^{\circ}$ at $M=0.1,0.25^{\circ}$ at $M=0.2$, $0.4^{\circ}$ at $M=0.3$, and $0.6^{\circ}$ at $M=0.4$. The largest deviations occurred at low Mach number due to the small measured phase difference.

Figures 40 and 41 show comparisons between the measured and theoretical angle correction at two additional downstream locations. The frequency dependence at $M=0.4$ is shown in part (a) and (b) of each figure while the Mach number dependence at a fixed frequency of $5 \mathrm{kHz}$ is shown in part (c) of each figure. This limited frequency and Mach number study was conducted to verify the trends obtained in figure 39. In all cases, the agreement between theory and experiment is good. The absolute difference between theory and data points was, in all cases, less than $2^{\circ}$ at $\theta_{\mathrm{m}}=37.5^{\circ}$.

Considering now off-axis source locations, figure 42 is a comparison between the measured and theoretical angle correction as a function of openjet Mach number and acoustic source frequency. The test conditions are identical to those evaluated in the on-axis geometry presented in figure 39. This approach permitted assessing the capability of the theory to predict changes in source-to-shear layer separation distances. The results for $\mathrm{f}=\mathrm{I} \mathrm{kHz}$ are not included due to the large scatter in the data.

Comparisons between the measured and theoretical angle corrections in figure 42 indicate good agreement at angles close to the jet axis. However, a consistent discrepancy occurred at large measurement angles for all frequencies and Mach numbers above $M=0.1$. A detailed error analysis of the experimental procedure was conducted to isolate the origin of the discrepancy. First, the sensitivity to assumed changes in $h$ was evaluated analytically. Second, the sensitivity to errors in the measure wavefront propagation 
angles was evaluated. Both analyses indicated that the resulting corrections to the $\theta_{\mathrm{m}}$ versus $\theta_{\mathrm{c}}$ curves were smaller than the discrepancies noted in figure 42 . It should be noted that the shear layer was not required to be in the far field of the acoustic source. This is discussed in the Review section of the chapter titled Theoretical Formulation of the Refraction Problem.

The zero thickness shear layer assumption was also questioned. Refraction theory was used to trace an acoustic ray propagating from the source to the outer edge of the finite thickness shear layer. The shear layer was modeled as a series of parallel vortex sheets with the refraction theory applied across each sheet. The shear layer crossing point, $X_{0}$, and wavefront propagation angle outside the flow were determined by this detailed analysis. The resulting measurement angle $\theta_{m}$ was then calculated and compared to the value predicted by the zero thickness calculation in figure 42. The difference was found to be insignificant.

Mean velocity profiles between the speaker aperture and the shear layer were also measured as part of the experimental procedure error analysis. The objective was to check for flow-field distortion due to the finite source size. The results indicated at most a 5 percent deviation from a uniform velocity profile measured in the absence of the speaker. Failure to obtain agreement at an off-axis source position having small $h / R_{O}$ is not presently understood.

To further investigate the observed disagreement between theory and experiment for the of $f$-axis case, additional measurements were performed with the source at the same axial location $\left(\mathrm{X} / \mathrm{R}_{\mathrm{O}}=1.33\right)$ but displaced in the opposite direction from the jet centerline $\left(h / R_{0}=1.44\right)$. Here the source and far-field microphone positions correspond to Test Configuration 5. Shown in figure 43 is a comparison between the measured and theoretical angle correction at selected Mach numbers and acoustic source frequencies. The agreement between theory and experiment is observed to be good at all operating conditions.

\section{Summary and Evaluation}

A theory capable of predicting the circular open jet refraction angle changes for an arbitrary source type, on-axis source position, and far-field microphone position was validated. Data obtained at three on-axis source positions, four source frequencies and four Mach numbers between $M=0.1$ and 0.4 verified the theoretical Mach number dependence and the frequency independence. Excellent agreement between theory and experiment verified 
that the lip-line vortex sheet model is an adequate representation of the finite thickness shear layer. Axial variations in the mean shear layer properties and divergence of the flow field are therefore not needed in the theoretical formulation.

Off-axis theory was validated for a source position having a large value of $h / R_{0}$. Failure to obtain agreement at an off-axis position having a small value of $h / R_{o}$ is presently not understood and will require additional research in the future. 


\title{
EXPERIMENTAL ASSESSMENT OF REFRACTION \\ AMPLITUDE CORRECTION THEORY
}

\author{
Experimental Approach
}

The objective of this investigation was to measure the amplitude change associated with an acoustic-wave propagating through an open jet shear layer. Measurements were conducted at the same open jet Mach numbers and acoustic source frequencles investigated in the refraction angle correction experiment. Similar test conditions were investigated since the angle correction was needed to define the refracted ray path on which the amplitude correction was measured.

The refraction amplitude change across the shear layer was assessed using the constant sideline measurement geometry shown in figure 23. Acoustic source sound pressure levels were measured inside and outside the open jet. The microphone location inside the open jet colncided with a ray propagating at a selected angle $\theta_{c}$. The far-field microphone was situated on the same acoustic ray except that the ray path was now refracted by the shear layer to the new propagation angle, $\theta_{t}$, shown in figure 23 . The intersection of the refracted ray with the sideline determined the measurement angle, $\theta_{m}$, which defined the far-field microphone coordinates.

A constant sideline geometry was selected for this experiment instead of the constant radius geometry previously described in the angle correction experiment. This choice was based on the large refraction amplitude correction which occurs in the sideline geometry. The magnitude of the correction is evident in figure 44 where the theoretical refraction amplitude corrections are compared for a constant sideline and constant radius microphone geometry at $M=0.4$. The larger corrections for the sideline geometry were expected to improve the accuracy of the experiment relative to the $\pm 0.2 \mathrm{~dB}$ error band expected from the signal enhancement measurement technique described below.

All figures presented in this section show the experimentally determined amplitude correction plotted as a function of $\theta_{c}$. This angle, instead of $\theta_{\mathrm{m}}$, was selected as the principle variable in the experiment since this permitted the in-flow microphone station to be held fixed as the open jet Mach number increased, thereby, facilitating the measurement procedure. Recall that angle $\theta_{\mathrm{m}}$ can be determined from angle $\theta_{\mathrm{c}}$ using the appropriate refraction angle correction curves in figure 24 or 28. 
The refraction amplitude correction converts sound pressure level measurements at the observer station (0) in figure 23 to the constant sideline station (D) on the non-refracted path. The corrected sound pressure level represents an equivalent measurement at station $D$ in the absence of the shear layer.

The refraction amplitude correction was experimentally assessed using acoustic source sound pressure level measurements inside and outside the open jet test section. The microphone positions were defined by $\mathrm{m}_{3}$ and $\mathrm{m}_{4}$ in figure 10 with the latter station being equivalent to the observer station (0) in figure 23. The equation linking the measured sound pressure levels and the calculated refraction amplitude correction between stations $(0)$ and (D) in figure 23 will now be derived.

Mean-square sound pressure levels measured inside and outside the open jet were represented by the function $\overline{P_{3}{ }^{2}\left(\theta_{c}, f, M\right)}$ and $\overline{P_{4}{ }^{2}\left(\theta_{m}, f, M\right)}$. The amplitude correction function, $\operatorname{AMP}\left(\theta_{c}, f, M\right)$ was then determined from the logarithmic expression

$$
A M P=10 \log \frac{\overline{P_{3}^{2}}}{\overline{P_{4}^{2}}}-10 \log \left(\frac{S D}{S m_{3}}\right)^{2}
$$

Here the ratio, $\overline{\mathrm{P}_{3}{ }^{2}} / \overline{\mathrm{P}_{4}{ }^{2}}$, represents the measured amplitude change on the refracted path. This change was compared with the calculated inverse squarelaw amplitude change on the non-refracted path. The non-refracted path change is represented by the ratio of the source-to-microphone distances in the second term on the right side of equation 17.

The mean square sound pressure level ratio, $\overline{\mathrm{P}_{3}{ }^{2}} / \overline{\mathrm{P}_{4}^{2}}$, could not be measured directly because the acoustic source signal consisted of a toneburst. Instead, the acoustic pressure wave-amplitude was measured. Letting $\mathrm{B}_{3}$ and $\mathrm{B}_{4}$ represent the amplitude of the periodic signal during a tone burst, the mean square sound pressure level was then replaced by the ratio $\mathrm{B}_{3}^{2} / \mathrm{B}_{4}^{2}$.

Parameters, $\mathrm{B}_{3}$ and $\mathrm{B}_{4}$, were measured using a time delayed signal enhancement technique which captured the tone burst waveform arriving at either the in-flow or far-field microphone. The details of this approach can be described using figure $45 \mathrm{a}$. Recall that the repetitive discretetone burst was provided by a function generator which powered the acoustic driver. The generator also provided a variable delay pulse signal which triggered the signal enchancement circuit to capture the tone burst. The delay time, $\tau$, was selected to correspond to the propagation time between the acoustic source and the individual microphones. The delay 
was adjusted until the tone burst appeared in the sampling time window of the signal enhancement circuit.

The circuit schematic in figure $45 \mathrm{a}$ shows how either the in-flow microphone $\left(m_{3}\right)$ or the far-field microphone $\left(m_{4}\right)$ were selected for signal enhancement. Note that by using a common filter and amplifier, differences in sensitivity were restricted to differences in the cartridge and preamplifier sensitivities of the separate microphones. These differences were calibrated daily using a pistonphone and the autocorrelation mode on the analyzer used for signal enhancement.

Figure 45b illusirates a segment of the tone burst signal obtained from either the in-flow or far-field microphone after signal enhancement. Sample increment times for the 400 point signal enhancement analysis were selected to give a maximum of 5 maxima of the periodic signal with the analyzer time window defined by $(\tau, \tau+\Delta \tau)$. By limiting the number of cycles, each cycle was described by a minimum of 80 discrete points. This was considered sufficient to resolve the amplitude of the discrete tone signal during the tone burst.

The time trace in figure 45b shows a modulated sine wave with a slowly increasing amplitude. In contrast, the function generator output signal (fig. 45a) shows a constant amplitude signal applied to the acoustic driver. The difference between the two signals which was found to be a function of the acoustic driver dynamic response, would appear to have an impact on the equality, $\overline{\mathrm{P}_{3}{ }^{2}} / \overline{\mathrm{P}_{4}{ }^{2}}=\mathrm{B}_{3}{ }^{2} / \mathrm{B}_{4}{ }^{2}$. This expression assumes a constant discrete tone amplitude during the tone burst. The modulation was, however, identical at the in-flow and far-field microphones since it originated at the acoustic driver. The acoustic pressure ratio could therefore be replaced by the ratio of the peak pressure associated with the corresponding cycle at the in-flow and far-field microphone station. Hence

$$
\frac{\overline{\mathrm{P}_{3}^{2}}}{\overline{\mathrm{P}_{4}^{2}}}=\frac{\mathrm{B}_{3,1}{ }^{2}}{\mathrm{~B}_{4,1^{2}}}=\frac{\mathrm{B}_{3,2}{ }^{2}}{\mathrm{~B}_{4,2^{2}}}=. \cdot
$$

The second subscript in each term identifies the corresponding cycle in either the in-flow or far-field microphone time trace. For example, if figure $45 \mathrm{~b}$ represented the far-field microphone, the amplitude of the fifth maximum is designated by the term $\mathrm{B}_{4}, 5^{\circ}$. To ensure sufficient accuracy, the pressure ratio at a minimum of 4 peaks in the time signal were averaged and a standard deviation was also calculated. Hence

$$
\frac{\overline{P_{3}^{2}}}{\overline{P_{4}^{2}}}=\frac{1}{n} \sum_{i=}^{n} \frac{B_{3, i}^{2}}{B_{4, i}{ }^{2}}, n \geq 4
$$


Both positive and negative pressure peaks were used in the summation in equation 19. Additional cycles needed for the summation were obtained by applying an additional delay time at the analyzer resulting in the time window $(\tau+\Delta \tau, \tau+2 \Delta \tau)$ shown in figure $45 \mathrm{~b}$.

At high frequencies, acoustic pressure amplitudes associated with the first few cycles in the time trace-were neglected since the amplitudes were small. Similarly, the last few cycles, which were generated by the acoustic driver after the termination of the tone, were also neglected. The duration of the tone burst was usually limited to 10 cycles of the discrete tone at the acoustic driver input. This provided a starting point and termination point in the signal enhanced time trace. With the aid of these two reference points the corresponding peaks at the in-flow and far-field microphone stations were easily identified.

The success of the above described approach for capturing the tone burst is demonstrated at $M=0$ in figure 46. For this static operating condition the amplitude correction in equation 17 is zero since the acoustic rays are not refracted. The data in figure 46 indicates a $\pm 0.2 \mathrm{~dB}$ deviation from the theoretical prediction. $A \pm 0.2 \mathrm{~dB}$ error band $\mathrm{can}$, thus, be assigned to all experimental data points.

For $M=0,0.1$, and 0.2 , the tone burst signal was easily detected by the far-field and in-flow microphones. As the open jet Mach number was increased further, the facility random background noise began to dominate the acoustic signal arriving at the far-field microphone. Similarly, the in-flow microphone signal was dominated by microphone self noise. To overcome these signal-to-noise limitations, the number of ensembles in the signal enhancement was increased as the Mach number increased. The longer sampling times at the higher Mach numbers precluded recording on analog tape the simultaneous in-flow and far-field microphone signals at each angular measurement station. Consequently, the two microphone signals were processed sequentially using the circuitry shown in figure $45 \mathrm{a}$. The acoustic driver output amplitude remained constant during the long sampling time required to separately analyze the in-flow and far-field microphone signals. This was verified by repeating the signal enhancement to obtain a second measurement of the tone burst.

The success of the signal enhancement technique at high Mach numbers is illustrated in figure 47 . The curves represents the tone burst signals recovered from the far-field $\left(\theta_{\mathrm{m}}=44^{\circ}\right)$ and in-flow $\left(\theta_{\mathrm{c}}=30^{\circ}\right)$ microphones at $M=0.3$. Similar to figure $45 \mathrm{~b}$, the tone burst signals contain a modulated sine wave. The corresponding peaks at the in-flow and far-field microphone measurement stations are numbered using the scheme designated in equation 19. The ratios, $B_{3, i}^{2} / B_{4}^{2}, i$, in equation 19 are obtained from the ratio of the corresponding pressure amplitudes and the amplifier settings used during the data acquisition. 
The above described experimental approach assumed an omni-directional sensitivity for the in-flow microphone. Free-field correction curves provided by the microphone manufacturer indicated that the measured nose cone response was a function of the incidence angle, $\theta_{c}$, shown in figure 48. Since $\theta_{c}$ changed as the microphone moved along the in-flow traverse line the in-flow microphone directional sensitivity varied with traverse position. To eliminate any differences between the in-flow and far-field microphone response, a nose cone was also used on the far-field microphone. In addition, the far-field microphone was aligned parallel to the flow with the nose cone pointing upstream similar to the in-flow microphone orientation. Since the refraction amplitude correction was based on the decibel difference between the in-flow and far-field microphone sound pressure levels, the nose cone directional characteristics were, in concept, nullified.

The above conclusion is correct only if the incidence angle is identical at the in-flow and far-field microphone stations. Refraction, however, changed the acoustic ray incidence angle to $\theta_{t}$ outside the flow. This change was accounted for by using the experimentally determined nose cone directional sensitivity curves shown in figure 49. Curves were generated for the four tone burst frequencies used in the refraction amplitude correction experiment. The microphone response at different frequencies was plotted as a function of the acoustic ray incidence angle. All amplitudes in figure 49 were normalized relative to the zero incidence angle measurement. Stationarity of the acoustic source during the measurement was verified by a separate reference microphone. A typical reference microphone measurement is plotted in figure 49 for the $10 \mathrm{kHz}$ case.

Far-field microphone data was then corrected by the difference between the amplitude response measured at incidence angles $\theta_{c}$ and $\theta_{t}$. Since the transmitted angle, $\theta_{t}$, changed with Mach number, the directional sensitivity correction also varied with Mach number.

The response curves in figure 49 change rapidly for incidence angles greater than $90^{\circ}$. These features differ from the nose cone free field response curves provided by the microphone manufacturer. The discrepancy is presently not understood. Other investigators have, however, observed similar discrepancies.

It should be noted that using a standard grid on the far field microphone would result in a larger directional sensitivity correction. This is because of the vastly different response for a standard microphone grid and the nose cone used on the in-flow microphone. Ahuja, et al. (ref. 15) used a nose cone 
and standard grid microphone combination but did not include the Mach number directional sensitivity response corrections. This raises questions about the accuracy of their measurements.

Comparison of Measured and Theoretical Refraction Amplitude Correction

Downstream Quadrant, On-Axis Source Location - Comparisons of refraction amplitude correction theory and experiment are first presented for the case of an on-axis acoustic source location. The Mach number dependence of the refraction amplitude correction is assessed in figure 50 over the range $M=0.1$ to 0.4 . The experimental results were obtained using a fixed acoustic source frequency of $\mathrm{f}=1.25 \mathrm{kHz}$. Figure $50 \mathrm{~d}$ also includes the measured correction at a source frequency of $\mathrm{f}=2.5 \mathrm{kHz}$. Error bars designate the limits of the standard deviation calculated from the separate amplitude ratios in equation 19. Error bars smaller than the symbol size were not included.

Source and microphone positions in figure 50 correspond to Test Configuration 6 listed in Table II. Measurements at angles greater than $80^{\circ}$ could not be obtained for this source location due to line of sight interference by the open jet nozzle lips. Moving the source downstream to $\mathrm{X} / \mathrm{R}_{\mathrm{O}}=2.66$ permitted increasing the upstream quadrant measurement angle to $\theta_{c}=120^{\circ}$. These results correspond to Test Configuration 7 and are presented in the following subsection.

The agreement between theory and experiment in figure 50 is considered good over the complete angle range and Mach number range. As expected from theory the refraction amplitude correction is a minimum near $\theta_{\mathrm{c}}=90^{\circ}$.

At angles close to the open jet axis, the amplitude correction increases in magnitude with increasing Mach number. This is demonstrated in figure 51 where the amplitude correction for a ray propagating at $\theta_{c}=30^{\circ}$ is plotted as a function of increasing Mach number.

Figures 50 and 51 verify the theoretically predicted Mach number dependence of the amplitude correction at a fixed source frequency. The theory also predicts that the refraction amplitude correction is independent of frequency. This theoretical result is assessed in figure 52, where the Mach number is fixed at $M=0.3$ and the frequency increased from $\mathrm{f}=1.25 \mathrm{kHz}$ to $10 \mathrm{kHz}$. At $\mathrm{f}=1.25 \mathrm{kHz}$ and $2.5 \mathrm{kHz}$, the agreement between theory and experiment is shown to be good. At $f=5 \mathrm{kHz}$ and $10 \mathrm{kHz}$, however, a significant disagreement between theory and experiment occurs at angles close to the jet axis. For example, figure $52 \mathrm{~d}$ shows approximately a $10 \mathrm{~dB}$ difference between theory and experiment at $\theta_{c}=25^{\circ}$. These results appeared to conflict with those of Ahuja, et al. (ref. 15) who found reasonable experimental agreement 
with a theory similar to that developed in the present study. Experimental assessment in the study by Ahuja employed a broadband noise source.

The discrepancy in figures $52 \mathrm{c}$ and $52 \mathrm{~d}$ was postulated to be due to frequency and directional scattering of the discrete tone burst energy by the turbulence in the open jet shear layer. Since the narrowband filter in figure 45a was centered at the discrete tone frequency, energy scattered into adjacent frequency bands was not measured by the far field microphone. Similarly energy scattered in directions different from the refracted propagation angle, $\theta_{t}$, would not be measured by the far-field microphone. Thus, the measured sound pressure level, $\overline{\mathrm{P}_{4}}$, in equation 17 would be smaller than anticipated resulting in a larger ratio for $\overrightarrow{\mathrm{P}} \frac{2}{3} / \frac{2}{4}$. The difference term on the right side of equation 17 would then provide a positive amplitude correction similar to that observed at $\theta_{c}=25^{\circ}$ in figure $52 \mathrm{~d}$.

The importance of the scattering mechanism was investigated in a preliminary experiment conducted during the first research program reported in reference 1 . The results indicated a significant loss in discrete tone sound pressure levels at high frequencies and high Mach numbers for sound propagating through the open jet shear layer. The magnitude of the loss was comparable to the amplitude changes in figure $52 \mathrm{~d}$, and increased as Mach number and the ratio of the shear layer thickness to acoustic wavelength increased.

A brief discussion of the parameters controlling the scattering phenomenon will be presented here to help in the interpretation of the discrepancies in the refraction amplitude correction data. Additional details are given in the section on turbulence scattering. The open jet Mach number controls the absolute turbulence intensity in the shear layer. In the absence of turbulence, scattering would be nonexistent. The shear layer thickness controls the local turbulent eddy size. This is an important parameter since sound is scattered only by the component of turbulence which possesses a wave number comparable to the acoustic wave number. The shear layer thickness also controls the acoustic propagation path length in the turbulent medium. A larger path length results in more interactions between the acoustic wavefront and the turbulent eddies which scatter the sound.

To isolate those test conditions in which scattering effects contaminated the refraction amplitude correction data, the difference between theory and experiment was plotted as a function of $\mathrm{L} / \lambda$ and Mach number. Here $\mathrm{L}$ is the propagation path length in the turbulent shear layer. The results are shown in figure 53 for two different propagation directions. At angle $\theta_{c}=30^{\circ}$, where the acoustic ray propagates obliquely through the shear layer, the path length was estimated to be $0.31 \mathrm{~m}$. This calculation was based on the simple 
expression, $L=\delta / \sin \theta_{C}$, where $\delta$ corresponds to the local shear layer thickness at the intersection of the acoustic ray and the open jet lip line. Figure 53a shows that for low frequencies, where $L / \lambda$ is approximately unity, scattering was negligible for all Mach numbers. But as $L / \lambda$ approached a value of 10 scattering became significant for increasing Mach numbers. In this case the number of scattering interactions between an acoustic wave front and the turbulent eddies in the shear increased by a factor of approximately 10 resulting in dramatically stronger scattering.

When the acoustic ray penetrated the shear layer at $\theta_{c}=80^{\circ}$, L was approximately equal to the shear layer thickness, $\delta$. The acoustic measurements in this case showed a negligible difference between refraction amplitude correction theory and experiment over the complete frequency and Mach number range. As expected the ratio of $L / \lambda$ was small for all operating conditions. This provided further support to the supposition that discrepancies between theory and experiment were due to turbulence scattering of acoustic energy.

To provide a conclusive confirmation of the presence of discrete tone scattering in the amplitude correction experiment, measurements were repeated using random noise. The experiment was performed at $10 \mathrm{kHz}$ where the largest discrepancy between theory and experiment was observed. In-flow and far-field sound pressure level measurements were obtained at $M=0.2$ using a $1 / 3$ octave band filter centered at $10 \mathrm{kHz}$. For this operating condition, the acoustic driver output power dominated over the in-flow microphone selfnoise and the open jet background noise by almost $10 \mathrm{~dB}$. The experimental amplitude correction, calculated from equation 17 is shown in figure 54. The results indicate good agreement with theory over the complete angular measurement range when using random noise. In contrast, figure 54 shows a significant discrepancy between theory and experiment when using the tone burst noise source. Since scattering would be expected to be much stronger for pure tones than random broadband noise, the above results confirmed the hypothesis that scattering is responsible for the observed disagreement between theory and experiment in figure 52. When the good agreement for the random noise result at $10 \mathrm{kHz}$ is combined with the good agreement at $\mathrm{f}=1.25$ $\mathrm{kHz}$ and $2.5 \mathrm{kHz}$ in figure 50 , the frequency independence of the refraction amplitude correction is confirmed.

Figure 54 suggests that random noise should be used for all operating conditions in the amplitude correction experiment. Random noise, unfortunately, creates an immediate signal-to-noise limitation at high Mach numbers. The major disadvantage of this approach is the distribution of acoustic source output power over a large portion of the spectrum resulting in low acoustic levels per unit Hertz. These low levels are masked by the microphone self-noise at the in-flow measurement station and the open jet background noise at the far field station. Consequently, the signal-to-noise 
ratio is low. One method for circumventing this background noise limitation employs the cross-spectrum measurement techniques used by Ahuja, et al. (ref. 15). The measurement errors associated with this approach, however, precluded using the concept. This will be discussed further in the subsection titled "Comparison with Other Investigators". In addition, the ability of the technique to recover the source signals was questionable at the highest Mach number of $M=0.4$ used in the present study. Based on scaling laws, the facility background noise due to the open jet noise would increase by $12 \mathrm{~dB}$ relative to the $\mathrm{M}=0.27$ maximum open jet velocity investigated by Ahuja. For these reasons, the discrete tone approach was used throughout the present study.

Continued application of the discrete tone signal in the amplitude correction experiment required identifying those test conditions in which scattering contaminated the data. These cases were easily recognized by the large positive amplitude correction at angles close to the open jet axis. Comparisons between theory and experiment are not presented for these cases. The remaining test conditions for which turbulence scattering was negligible are shown in figures 55 and 56. As before, the agreement between theory and experiment is considered good with the exception of a few angles below $\theta_{\mathrm{c}}=$ $35^{\circ}$ in figure 56. When the results in figures 55 and 56 for $\mathrm{f}=2.25$ and $5 \mathrm{kHz}$ are combined with the good agreement at $1.25 \mathrm{kHz}$ ( $\mathrm{fig} .50$ ) and $10 \mathrm{kHz}$ (fig. 54) the frequency independence of the refraction amplitude correction is verified.

Upstream Quadrant, On-Axis Source Location - Refraction amplitude correction tests were conducted for a second on-axis source station described by Test Configuration 7 in Table II. The source location, which corresponded to $X / R_{0}=2.66$, was downstream of the station described previously. This permitted making amplitude correction measurements in both the downstream and upstream quadrant.

Comparisons of refraction amplitude correction theory and experiment are presented in figure $57 \mathrm{a}$ for $\mathrm{f}=1.25 \mathrm{kHz}$ and $\mathrm{M}=0.3$. A limited number of measurements were made at angles $\theta_{c}<90^{\circ}$ based on the good agreement obtained at $\theta_{c}=30^{\circ}$ and $40^{\circ}$ where the amplitude correction is the largest. Figure $57 a$ also provlded the first confirmation of the refraction amplitude correction for measurement angles in the forward arc corresponding to $\theta_{c}>90^{\circ}$.

As the source frequency increased, disagreement between theory and experiment became prevalent as shown by the $\mathrm{f}=5 \mathrm{kHz}, \mathrm{M}=0.3$ case in figure 57b. When compared to the measurements described previously in figure 52c the discrepancy increased as the acoustic source moved downstream. This trend is easily explained by the increase in shear layer thickness as the source position moves downstream. The propagation path through the turbulent shear layer then increases resulting in stronger scattering effects. 
To assess the Mach number dependence of the amplitude correction in the absence of shear layer scattering, the lowest acoustic source frequency was selected. Figure 58 shows the comparison of theory and experiment over the range $M=0.1$ to 0.4 with $f=1.25 \mathrm{kHz}$. Measurements at $M=0.3$ were made in 5 degree increments to assess the detailed shape of the correction curve. Selected angles were investigated at the other Mach numbers to verify the theory at the extreme measurement angles. In each case, the agreement between theory and experiment is considered good. The theoretical dependence of the amplitude correction on Mach number was, therefore, verified.

The good agreement between theory and experiment at the two different axial source stations presented in figures 50 and 58 also verified that the refraction amplitude change is independent of shear layer thickness. This independence of thickness in addition to shear layer divergence confirms a previous theoretical prediction reported by Amiet in reference 10. The lip line vortex sheet model employed in the present theory is, therefore, an adequate representation of the finite thickness shear layer.

To assess the frequency independence of the refraction amplitude correction theory in the upstream quadrant, the Mach number was held fixed and the acoustic source frequency was changed. The experimental results shown in figure 59, indicate good agreement at $\mathrm{f}=1.25 \mathrm{kHz}$ as well as $5 \mathrm{kHz}$. The good agreement at $5 \mathrm{kHz}$ contrasts the results in figure $52 \mathrm{c}$ where significant disagreement between theory and experiment occurred for angles close to the open jet axis. At the highest frequency of $10 \mathrm{kHz}$, figure $59 \mathrm{c}$ indicates a maximum disagreement of only $3 \mathrm{~dB}$ compared to a $10 \mathrm{~dB}$ difference in figure 52d. The improved agreement for the 5 and $10 \mathrm{kHz}$ source frequencies in the upstream quadrant was due to weaker scattering of sound by shear layer turbulence. This occurred because the ratio $\mathrm{L} / \lambda$ was smaller. For example, with $\mathrm{X} / \mathrm{R}_{\mathrm{o}}=0.53$ and $\theta_{\mathrm{c}}=30^{\circ}, \mathrm{L} / \lambda=9$ at $\mathrm{f}=10 \mathrm{kHz}$. In comparison, for $\mathrm{X} / \mathrm{R}_{\mathrm{o}}=2.66$ and $\theta_{\mathrm{c}}=120^{\circ}, \mathrm{L} / \lambda=4$. Scattering effects would then be weaker at $\theta_{c}=120^{\circ}$ resulting in the improved agreement between theory and experiment. These trends are discussed further in the section titled Turbulence Scattering of Sound Experiments.

Off-Axis Source Location - The refraction amplitude correction was also investigated at an off-axis source location defined by Test Configuration 8 in Table II. The axial position, defined by the parameter, $x / R_{O}$, corresponded to the source position investigated in Test Configuration 6 . The off-axis location, defined by the parameter, $h / R_{O}$, provided a source to shear layer separation distance larger than one jet radius. Source locations with $h / R_{0}<1$ were not investigated because of the failure to obtain agreement between refraction angle correction theory and experiment at this test condition. This was discussed in detail in the section titled Experimental Assessment of Refraction Angle Correction Theory. 
Figure 60 shows comparisons between the measured and theoretical amplitude correction at two acoustic source frequencies for $M=0.3$. The good agreement at the lowest frequency, where scattering is the weakest, confirms the off-axis dependence of the amplitude correction.

\section{Comparisons With Other Investigators}

Few experimental studies exist documenting the shear layer refraction amplitude correction. Candel, et al. described the experimental assessment of a refraction theory but provided little documentation to verify the dependence on frequency, Mach number, and acoustic source position. Ozkul and $\mathrm{Yu}$ (ref. 16) also evaluated the refraction amplitude correction for an on-axis acoustic source. Their experiment was limited to a maximum Mach number of $M=0.1$. The measurements showed good agreement with the theory reported earlier by Amiet in reference 7. This same theory also formed the basis for the analytical extension to off-axis acoustic source geometry derived in the present study.

Ahuja, et al. recently reported an experimental assessment of a separate refraction theory developed as part of the work reported in reference 15. It is worthwhile to clarify the relationship between the present refraction theory and that presented by Ahuja, et al. before discussing any experimental comparisons.

The refraction theory reported in reference 15 was limited to on-axis source locations. The angle correction segment of the analysis is exactly that given earlier by Amiet (ref. 7) and Jacques (ref. 8) for a zero thickness shear layer. The general analysis in the section titled Theoretical Formulation of the Refraction Problem reduces to that given in reference 10 for the special case of an on-axis acoustic source.

The general amplitude correction theory in the present study assumes a zero thickness shear layer. In contrast, the on-axis analysis used by Ahuja assumes a shear layer thickness which is infinite compared to the acoustic wavelength. The difference between these two approaches for an on-axis source was given earlier by Amiet (eq. (14) of ref. 10) and is quite small over most of the angular range, as shown in figure 61. At the extreme measurement angles (near the zone of silence and near the upstream axis) where the difference between the two approaches becomes significant, neither result would be expected to be accurate since each approach neglects the multiple reflections which occur in these regions. Thus, in the range of practical use, the approach of Ahuja gives results which differ little from those previously given by Amiet (ref. 7) and generalized here to treat the case of an off-axis source. 
Verification of the amplitude correction theory in the present study was considered to be more accurate than the experimental assessment reported by Ahuja, et al. in reference 15. This is because the errors associated with the signal enhancement method were smaller than those encountered in the cross-spectrum measurement technique. This point will now be discussed further.

Recall that the cross-spectrum approach was employed by Ahuja et al. to circumvent the signal-to-noise limitation encountered when using a random noise acoustic source. The method employed a reference microphone adjacent to the acoustic source. Microphone signals were then processed to give the reference-to-in-flow microphone and reference-to-far-field microphone crossspectra. The experimentally determined amplitude correction was then obtained from equation 4-3 in reference 15 ,

$$
\text { - } \quad C_{F_{m}}=\frac{\left|G_{A C}(f)\right|^{2}}{\left|G_{A B}(f)\right|^{2}} \frac{R_{r T}{ }^{2}}{R_{r o} R_{r o}}
$$

Here the parameters, $G_{A C}$, and $G_{A B}$, represent the square of the modulus of the pressure cross-power spectral densities. The ratio of these two spectra is similar to the ratio of the mean-square acoustic pressures in equation 17 of the present report. The remaining terms in equation 20 represent different source-to-microphone distance corrections.

Measuring the decibel difference between $G_{A C}$ and $G_{A B}$, provided the experimental input to the refraction amplitude correction calculation in equation 21 . The present authors feel that the scatter in the individual crossspectrum curves makes it difficult to accurately determine the decibel difference. Figure 62 shows the cross-spectra reported by Ahuja in figure 4.41 of reference 15. This figure was obtained by photographically enlarging the original curve by a factor of 4 and tracing the resulting spectra. Frequencies below $1 \mathrm{kHz}$ and above $14 \mathrm{kHz}$ are not included due to the poor quality of the original curve published in the report.

As noted in reference 15 , the cross-spectra curves are displayed $10 \mathrm{~dB}$ apart to show the approximately constant amplitude difference as a function of frequency. A close examination of figure 62, however, indicates that the difference between the curves is not constant. This is because details found in one curve are not necessarily present in the second curve. For example, at $f=2.5$ and $3 \mathrm{kHz}, G_{A B}(f)$ has two minima which fail to appear in the curve for $G_{A C}(F)$. Consequently, the amplitude difference is estimated as 14.2 and $13.6 \mathrm{~dB}$ instead of $10 \mathrm{~dB}$. Similar comparisons are provided across the entire spectrum in figure 62 . 
Fluctuations of several decibels in the logarithmic difference, $G_{A C}{ }^{-G_{A B}}$, makes it difficult to assign a single value to the amplitude difference between the two cross-spectra curves. Furthermore, the fluctuations make it difficult to conclude, as Ahuja, et al. did, that the amplitude correction is frequency independent. These observations raise the question of uncertainty in the experimental approach. The fluctuations in figure 62 suggests a $1 \mathrm{~dB}$ error is reasonable and possibly the error is larger. In comparison, the largest refraction amplitude correction measured by Ahuja, et al. was approximately $3 \mathrm{~dB}$. This occurred at $\theta_{c}=40^{\circ}$ and $M=0.27 . \mathrm{A}$ comparison of the uncertainty with the largest amplitude correction measured demonstrates that errors associated with the cross-spectrum approach were significant. For this reason the cross-spectrum measurement technique was not employed in the present study. Instead, the signal enhancement technique with its $\pm 0.2 \mathrm{~dB}$ error was employed. Admittedly, this required using a discrete tone source signal which was sensitive to turbulence scattering. But the operating conditions in which scattering occurred were easily recognized without compromising the accuracy of the experiment.

\section{Summary and Evaluation}

Refraction of sound waves passing through an open jet circular shear layer results in significant amplitude changes at Mach numbers greater than $M=0.1$. Acoustic source directivity patterns are therefore changed resulting in erroneous conclusions being drawn from an experiment. The measured amplitudes at the far field microphone station can be corrected theoretically using the refraction amplitude correction analysis verified by the present experiment.

The experiment verified the ability of the theory to predict the circular open-jet refraction amplitude changes for an arbitrary acoustic source position and far-field observer position. The lip line vortex sheet model employed in the theory is, therefore, an adequate representation of the finite thickness shear layer investigated in the present study. Axial variations in the mean shear layer properties and divergence of the flow field are therefore not needed in the theoretical formulation. The Mach number dependence and frequency independence of the theory was also verified. 


\title{
TURBULENCE SCATTERING OF SOUND EXPERIMENTS
}

\author{
Formulation of the Problem
}

Measurements obtained during the refraction amplitude correction experiment indicated the occurrence of discrete tone scattering as sound propagated through the open jet shear layer. For example, the discrepancy between amplitude correction theory and experiment in figure 52 was traced to the presence of scattering. The objective of the experiment described in this section was to quantitatively assess the magnitude of turbulence scattering in the open jet. This was accomplished by monitoring the discrete tone broadening and the discrete tone amplitude reduction as sound propagated through the shear layer.

The experiment was conducted using the sideline microphone geometry described in the refraction amplitude correction experiment (figure 10). In-flow and far-field spectra were measured on the same acoustic ray path. The resulting spectra were compared after accounting for the refraction amplitude change, the inverse square-law decay between the two microphone stations, and the nose cone directional sensitivity characteristics. Differences between the discrete tone amplitudes in the two spectra provided a direct calibration of the magnitude of the discrete tone attenuation along the refracted ray path. The significance of tone broadening was determined from the spectrum shape.

Contrary to the tone burst method used in the refraction amplitude correction experiment a continuous discrete tone was used in the scattering experiment. This simplified the discrete tone spectrum acquisition and interpretation. To avoid contamination of the far-field microphone signal by sound reflected from the collector, the sideline distance was decreased to $\mathrm{y}=0.91 \mathrm{~m}$ so that the direct radiation arriving at the microphone dominated the reflected sound.

In addition to the experimental effort the existing turbulence scattering analysis of Lighthill (ref. 24) was modified to provide an estimate of the discrete tone amplitude reduction. The modified analysis was also used to drive an expression for predicting the onset of turbulence scattering. Experimental results were compared with the resulting analysis.

The analysis of Lighthill assumed single scattering. An alternate approach is to assume multiple scattering as in the analysis of Howe (ref. 25). Unfortunately, this approach neglects the time dependence of the turbulence so that there is no frequency scattering for a discrete tone signal. However, by adding mean flow to the analysis, the frozen turbulence restriction can be, removed as reported by Amiet in reference 26. The modified analysis assumed a Doppler shift due to the motion of the turbulence. In this way frequency scattering was introduced into the 
multiple scattering problem. Comparisons between the modified analysis of Amiet and the present experiment are provided.

\section{Parameters Controlling Scattering}

When a sound wave passes through a region of turbulence, it encounters both frequency and direction scattering. The first mechanism extracts energy from one frequency band and redistributes it in adjacent frequency bands. If the acoustic signal contains a discrete tone, the frequency scattering results in a broadened spectrum. The direction scattering on the other hand scatters sound to new angles resulting in a change of the discrete tone directivity pattern.

It is not the purpose of this section to give an original treatment of the scattering of sound by turbulence. The theoretical and experimental investigations conducted by the authors cited in the bibliography of references 24 and 25 are considered adequate. Instead, the present section will link the physical parameters controlling the scattering phenomenon with the results observed in the experiments.

The first parameter to be considered in the turbulence scattering phenomenon is the magnitude of the turbulence intensity. The general trend established by the various theoretical analyses suggests that turbulence scattering increases as the absolute value of the intensity increases. An increase in the open jet Mach number, therefore, creates stronger scattering.

Turbulent eddy size is another parameter which controls the scattering mechanism. Direction scattering may be considered to be caused by a reflection of sound from the turbulent eddies similar to that of Bragg reflection where $X$ rays are scattered from a crystal. For the case of sound the acoustic wave is only reflected by the component of turbulence which possess a wave number comparable to the acoustic wave number (ref. 24).

Directional scattering is a prerequisite to frequency scattering. The latter phenomenon is observed as a Doppler shift of the discrete tone by the turbulent eddy. The resulting Doppler shift can be explained by the schematic in figure (63a). Consider the direction scattering of sound to be modeled as a two step process consisting of an initial absorption of the sound propagating in direction, $\theta+\theta_{5}$. Angle $\theta_{s}$ defines the scattering angle while $U_{\text {f }}$ represents the eddy convection velocity. The resulting Doppler shift in the new propagation direction is then given by the equation

$$
\Delta f=f_{0} U_{c} \frac{\left[\cos \left(\theta_{c}+\theta_{s}\right)-\cos \theta_{c}\right]}{\left[c_{0}-U_{c} \cos \left(\theta_{c}+\theta_{s}\right)\right]}
$$


It follows from equation 21 that the Doppler shift is zero along the ray path for which the scattering angle is zero.

Figure $63 a$ neglects the mean velocity gradient and the resulting refraction which takes place across the shear layer. Such velocity gradients, however, do not create a Doppler shift if the observer is stationed on the refracted ray path. Sound reaching the far-field microphone station (figure 63b) at the original source frequency then represents the portion of the acoustic energy which is not scattered out of the refracted ray path. Any reduction of the discrete tone amplitude measured at the observer station is then due to scattering of sound to a new direction given by $\theta_{t}+\theta_{S}$.

Monitoring the decrease in the tone amplitude at the acoustic source frequency provides a method for calibrating the direction scattering phenomenon. Admittedly, acoustic energy can arrive at the observer station after being direction scattered from other turbulent regions in the shear layer. This is illustrated by the ray initially propagating at angle $\theta_{c}^{\prime}$ in figure $63 \mathrm{~b}$. However, the direction scattered sound which now arrives at the observer station has a Doppler shifted frequency which is different from the original source frequency. Scattering volumes located downstream of the refracted ray path shear layer crossing-point, $x_{0}$, in figure $63 \mathrm{~b}$ result in a Doppler shift to lower frequencies based on equation 21 . In contrast, upstream scattering volumes cause a shift to high frequencies. This explains how tone broadening can occur in the spectrum at the observer station in the absence of a Doppler shift of the discrete tone center frequency. If the scattering is suffictently strong the resulting measured spectrum will be a broad hump with no perceptible discrete tone. In this case the initial energy in the acoustic wave front has been scattered to new directions and the measured spectrum represents the energy, Dopplershifted by the turbulence volumes upstream and downstream of station $\mathrm{X}_{0}$.

It should be emphasized that the far-field microphone in figure 63b will always sense some of the acoustic energy at the original source frequency. This is because for every microphone station, there exists a unique refracted path along which Doppler shifts are non-existent. If direction scattering is weak, the sound sensed at the microphone station is then dominated by the original discrete tone frequency. For strong scattering, the tone is not perceptible in the spectrum. It is replaced by a broad-band hump with a maximum at the original source frquency. The adjacent frequency bands now contain energy due to scattering of sound from turbulence volumes upstream and downstream of the refracted ray crossing point. 
The above described frequency and direction scattering effects become stronger as the acoustic propagation path length in the turbulent medium increases. This follows due to the increase in the number of interactions between the acoustic wavefront and the turbulent eddies in the flow.

\section{Discussion of Experimental Results}

Comparisons between the in-flow and far-field measured spectra are presented first for the case of sound radiating normal to the open jet axis from a source situated on the centerline at $x / R_{0}=0.53$. The acoustic source and microphone arrangement corresponded to Test Configuration 9 in Table III. The open jet Mach number dependence, and hence, shear layer turbulence intensity dependence is assessed in figure 64 over the frequency range of $\mathrm{f}=5 \mathrm{kHz}$ to $15 \mathrm{kHz}$. All far-field sound pressure level amplitudes are plotted relative to the in-flow measurement. The latter measurement was normalized to unity amplitude or zero decibels. A $50 \mathrm{~Hz}$ analyzer bandwidth was used for all spectrum measurements.

In-flow sound pressure levels in figure 64 were corrected for the refraction amplitude change and the corresponding inverse square-law decay between the in-flow microphone and the non-refracted sideline observer position (station $D$ in figure 23). In addition, corrections were also applied for the nose cone directional sensitivity characteristics determined from continuous discrete tone calibration curves similar to those in figure 49. The resulting spectra then corresponded to the sound pressure levels measured at the far-field microphone station in the absence of refraction and scattering. The far field spectra were then plotted relative to the corrected in-flow spectra. The peak amplitude difference between the two spectra calibrated the magnitude of the scattering effect.

Figure 64 shows a gradual decrease in the tone amplitude and a simultaneous broadening of the spectrum with increasing Mach number. The tone amplitude decrease demonstrates the occurrence of direction scattering of sound to new angles. Since these angles are not on the refracted ray path linking the in-flow and far-field microphone station, a decrease in tone energy is sensed at the observer station. Spectrum broadening occurs due to sound arriving at the measurement station from turbulence scattering volumes upstream and downstream of the acoustic ray cross-over point in the shear layer. The strength of the scattering phenomenon is seen to increase as the acoustic source frequency increases between figure $64 \mathrm{a}$ and $64 \mathrm{c}$. This follows since the acoustic wave length is approaching the size of the turbulent energy bearing eddies responsible for the scattering phenomenon.

To investigate the effect of increasing the propagation path length through the turbulent medium in addition to changing the turbulent eddy size, spectra were measured at different radiation angles given by $\theta_{c}=50^{\circ}$ 
and $30^{\circ}$. Here a decrease in $\theta_{c}$ results in an increase of the propagation path length, $L$, based on the approximation $L=\delta / \sin \theta_{c}$. In addition, the turbulence length scales were expected to increase since they are known to scale with the local shear layer thickness. THe results shown in figure 65 verify the previously described trend of increased scattering effects when the propagation path length increased. Both discrete tone amplitude attenuation and spectrum broadening become stronger as $\theta_{c}$ approaches the open jet axis. Thus, experiments limited to $\theta_{c}=90^{\circ}$ are not sufficient for determining the severity of the discrete tone scattering phenomenon. It should be noted that the turbulence intensity is approximately constant downstream of the open jet exit plane (figure 19). Thus, the trend observed here is not due to differences in the turbulence intensity at the acoustic ray shear layer crossing points associated with the different radiation angles, $\theta_{c}$.

To further investigate the effect of propagation path length changes on the scattering phenomenon, the acoustic source was shifted downstream to station $X / R_{0}=2.66$. The test geometry in this case corresponded to Test Configuration 10 in Table III. Figure 66 shows the open jet Mach number dependence over the frequency range of $\mathrm{f}=5 \mathrm{kHz}$ to $15 \mathrm{kHz}$ for sound radiating at $\theta_{c}=90^{\circ}$. When compared to the upstream source position results described earlier (figure 64) the tone broadening becomes stronger for downstream acoustic source positions. Figure 66 also shows a small increase in the discrete tone amplitude attentuation. This amplitude change is not as dramatic as the tone broadening change between the upstream and downstream source station. This is because only a small amount of scattered acoustic energy is needed to broaden the spectrum at the $-25 \mathrm{~dB}$ level in figure $66 \mathrm{c}$.

To further assess the propagation path length changes and the turbulent eddy size changes at this downstream source station measurements were also conducted at radiation angles other than $\theta_{C}=90^{\circ}$. The results shown in figure 67 for a range of frequencies at a fixed Mach number, $M=0.3$, indicate severe scattering for angles close to the open jet axis. The discrete tone peak amplitude at $\mathrm{f}=15 \mathrm{kHz}, \theta_{\mathrm{c}}=40^{\circ}$ has decreased by $10 \mathrm{~dB}$ relative to the $\theta_{c}=30^{\circ}$ measurement in figure 65 . Scattering effects are also stronger at the lowest frequency of $\mathrm{f}=5 \mathrm{kHz}$ when compared to the results shown for the upstream station in figure 64. In this case the shear layer thickness has increased sufficiently to provide large scale energy bearing eddies comparable in size to the acoustic wavelength.

Far-field spectra in figure 67 show less scattering at $\theta_{c}=120^{\circ}$ than at $\theta_{c}=90^{\circ}$ for $\mathbf{f}=5 \mathrm{kHz}$ and $10 \mathrm{kHz}$. At these frequencies the acoustic wavelength is considered large relative to the local shear layer thickness at $\theta_{c}=120^{\circ}$ resulting in weak scattering. A further increase in source frequency to $15 \mathrm{kHz}$ changes the ratio of wavelength to eddy size sufficiently to create stronger scattering at $\theta c=120^{\circ}$ than at $\theta_{c}=90^{\circ}$. The reversal is explained by the magnitude of the turbulence intensity vector. At $\theta_{c}=90^{\circ}$ 
the fluctuating velocity vectors coinciding with the acoustic rays arriving at this angle have approximately a single component in the radial direction. On the other hand, both axial and radial fluctuating velocity components combine to form the fluctuating velocity vectors coinciding with sound rays arriving at $\theta_{c}=120^{\circ}$. The turbulence vector is then larger in this direction of propagation. Assuming scattering to be related to the magnitude of the fluctuating velocity vector, scattering would be expected to be stronger at $\theta_{c}=120^{\circ}$. This argument can be generalized to explain the stronger effects at all angles for which $\theta_{c} \neq 90^{\circ}$.

Conservation of acoustic energy between the in-flow and far-field microphone is demonstrated in figure $67 \mathrm{c}$. Total acoustic energy arriving at the far-field microphone was obtained by integrating the spectra in the frequency domain. The resulting intensity, represented by the open-circle symbols, is approximately equal to the discrete tone amplitude at the in-flow microphone station. This suggests a two-step scattering process. First acoustic energy is scattered to new directions resulting in a decrease tone amplitude at the source frequency. Simultaneously, sound is scattered in the direction of the far-field microphone from the remainder of the open jet shear layer. This sound arrives at Doppler-shifted frequencies which broaden the spectrum. The integral of this energy is comparable to the energy decrease at the acoustic source frequency. The total energy in the spectrum, therefore, remains unchanged. Although this explanation satisfies the conservation of acoustic energy observed at the far-field microphone station, the authors recognize that it may be too simple an explanation.

Changes in the degree of scattering due to changes in acoustic source position are summarized in figure 68. Here the source frequency, radiation angle, and open jet Mach number were held fixed to isolate source position changes. The measurements demonstrate that turbulence scattering effects are more pronounced at downstream stations.

The degree of tone broadening and reduction in the peak amplitude of the far-field spectra in the previous figures is a function of the analyzer bandwidth. A large bandwidth, such as the $50 \mathrm{~Hz}$ value used in figures 64-68, narrows the spectrum and increases the peak amplitude. The latter effect occurs because the filter band can pass acoustic energy at the Doppler shifted frequencies surrounding the acoustic source frequency. In the case of strong tone broadening the sound pressure level associated with the filter band is large since it passes both the narrowband random energy and the discrete tone energy. On the other hand, decreasing the filter bandwidth to $1 \mathrm{~Hz}$ results in a lower sound pressure level at the peak position in the spectrum.

The above described trends will now be demonstrated using the experimental results obtained in the present investigation. Figure $69 \mathrm{a}$ contains the inflow and far-field spectra previously presented in figure $67 \mathrm{c}$ using a $60 \mathrm{~Hz}$ 
analyzer handwidth with $\mathrm{f}=15 \mathrm{kHz}$. Figure $69 \mathrm{~b}$ shows the spectrum obtained for the same test condition using a $0.25 \mathrm{~Hz}$ analyzer bandwidth. As expected the amplitude of the far-field sound pressure level measured with the 0.25 $\mathrm{Hz}$ filter had decreased significantly relative to the $50 \mathrm{~Hz}$ bandwidth measurement. Surprisingly, the $0.25 \mathrm{~Hz}$ spectrum also shows the remnant of the original discrete tone. This was not expected from the tone broadened spectrum in figure 69a. One advantage of the smaller bandwidth is, therefore, the ability to isolate the discrete tone in the presence of the broadened spectrum. The tone amplitude in figure $69 \mathrm{~b}$ is weak when compared to the narrowband random sound pressure levels at adjacent frequencies. This is demonstrated by integrating the random noise level in a $50 \mathrm{~Hz}$ band centered at the discrete tone in figure 69b. The random noise level in this case is approximated by the constant amplitude line. The total energy in the $50 \mathrm{~Hz}$ band, obtained by logarithmically summing the random noise and discrete tone, corresponds to the peak amplitude $(-8.5 \mathrm{~dB})$ of the broad-band peak in figure 69a. In comparison, the discrete tone by itself has a $-24 \mathrm{~dB}$ amplitude verifying that the major contribution to the spectrum in figure $69 \mathrm{a}$ is abtained from the broad-band noise in the $50 \mathrm{~Hz}$ filter band.

A $0.25 \mathrm{~Hz}$ bandwidth spectrum analysis was also conducted for the in-flow microphone to check for the presence of random noise in the measurement with the $50 \mathrm{~Hz}$ analyzer bandwidth. The detailed spectrum, shown in figure $69 \mathrm{~b}$, indicates a discrete tone $3 \mathrm{~dB}$ weaker than the amplitude measured in figure 69a. The difference between the two discrete tone amplitudes is due to the random noise shown in figure $69 \mathrm{~b}$. When the integral of this noise, approximated by a constant level of $-26.5 \mathrm{~dB}$, is added to the discrete tone amplitude, the total energy in figure $69 \mathrm{a}$ is recovered. In conclusion detailed comparisons of the discrete tone amplitude changes between the in-flow and far-field microphone should, therefore, use as small a bandwidth as possible.

Figures $70 \mathrm{a}$ and $70 \mathrm{~b}$ show the same far-field microphone spectra presented in figures $69 \mathrm{a}$ and $69 \mathrm{~b}$ with the addition of an absolute frequency scale on the horizontal axis. The spectrum in figure 70a has been normalized by the peak sound pressure level. Arrows identify the sound pressure level obtained from integrating the $0.25 \mathrm{~Hz}$ narrowband random spectrum as well as the discrete tone amplitude in figure $70 \mathrm{~b}$. For the $15 \mathrm{kHz}$ acoustic source frequency, the $50 \mathrm{~Hz}$ bandwidth measurement is dominated by random noise. If the acoustic source frequency were decreased, scattering would be expected to be weaker with the discrete tone comprising a larger portion of the sound pressure level measured in the far-field. This is demonstrated in figures $70 \mathrm{c}-$ $70 \mathrm{f}$ where the $50 \mathrm{~Hz}$ and $0.25 \mathrm{~Hz}$ bandwidth spectra are compared. Based on the trends observed in figure $70 \mathrm{a}, 70 \mathrm{c}$, and $70 \mathrm{e}$ a large analysis bandwidth (such as $50 \mathrm{~Hz}$ ) can qualitatively demonstrate the importance of the scattering phenomenon and identify trends associated with parametric changes. However, the large analysis bandwidth cannot provide quantitative measurements of the discrete tone amplitude reduction and, hence, the magnitude of the direction scattering. Such measurements can only be made using small (such as $0.25 \mathrm{~Hz}$ ) bandwidth spectra like those shown in figure $70 \mathrm{~b}, 70 \mathrm{c}$, and $70 \mathrm{f}$. 
A calibration of the magnitude of the direction scattering can be obtained from the amplitude difference between the in-flow and far-field $0.25 \mathrm{~Hz}$ bandwidth spectra like those in figure 69b. Figure 71 shows this difference for a range of frequencies and radiation angles, $\theta_{c}$. Changes in the latter parameter represent changes in the acoustic propagation path length through the turbulent shearlayer in addition to changes in turbulent eddy size. The discrete tone amplitude attenuation is seen to increase dramatically above $5 \mathrm{kHz}$. In comparison to figure 67 the attenuation is observed to be stronger when monitored with a $0.25 \mathrm{~Hz}$ bandwidth analyzer. In this case the actual discrete tone amplitude is measured instead of an integral of the sound pressure over a finite bandwidth.

\section{Prediction of Turbulent Scattering Effects}

In its present form the shear layer correction does not account for the scattering of discrete tone sound by shear layer turbulence. As shown by the refraction amplitude correction measurements, scattering presents a source of error, and some estimate should be made of its importance.

Lighthill (reference 24) gives an estimate of the energy E scattered in new directions from a unit volume of turbulence per unit time as

$$
E=\frac{8 \pi^{2} L_{1}}{\lambda^{2}} I \frac{\overline{\left(v_{i}\right)^{2}}}{c_{0}^{2}}
$$

$$
\begin{aligned}
& \text { where } \quad \begin{array}{ll}
\mathrm{I} & \text { Intensity of incident sound } \\
& =\text { wavelength of incident sound } \\
\frac{\left(\mathrm{v}_{1}{ }^{\prime}\right)^{2}}{2} & \text { mean-square velocity of turbulence } \\
\mathrm{L}_{1}= & \text { macro scale of turbulence in the direction of the } \\
& \text { incident sound } \\
\mathrm{c}_{0} & =\text { sound speed }
\end{array}
\end{aligned}
$$

This result does not assume any particular type of turbulence, but does assume that $\lambda / L_{1}$ is less than unity.

This result can be used to estimate the fraction of the incident energy which is direction scattered. Consider a volume of turbulence with an area normal to the incident beam of $A$ and thickness $L$. The energy incident per unit time is $A I$. The fraction $R_{S}$ of the incident energy which is scattered is

$$
R_{S}=\frac{A L E}{A I}=\frac{8 \pi^{2} L_{1} L}{\lambda^{2}} \frac{\overline{\left(V_{1}^{\prime}\right)^{2}}}{c_{0}^{2}}
$$


The Lighthill theory is a single scattering theory so the above relation is valid only for small $R$ values. However, it should be possible to obtain from it an indication of when scattering affects a significant fraction of the incident energy.

Equation 22 can be used to relate the scattering from two different test cases designated as (a) and (b). If $R_{S}$ is the same for two cases, the degree of scattering should be the same. Equation 23 gives

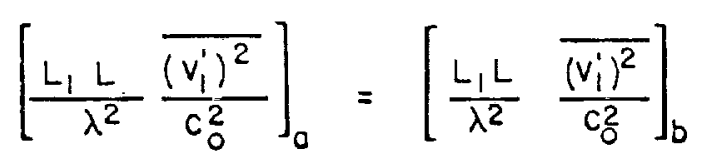

The mean square perturbation velocity, $\overline{\left(v^{\prime}\right)^{2}}$, will generally be proportional to the mean velocity $U$. Thus,

$$
\left[\frac{L_{1} L M^{2}}{\lambda^{2}}\right]_{0}=\left[\frac{L_{1} L M^{2}}{\lambda^{2}}\right]_{b}
$$

If the turbulent length scale is taken to be equal to the shear layer thickness,

$$
\left[\frac{L M}{\lambda}\right]_{a}=\left[\frac{L M}{\lambda}\right]_{b}
$$

Replacing the acoustic wavelength by the expression $\mathrm{f} / \mathrm{c}_{0}$ and assuming that the sound speed is the same in both cases gives the scaling parameter,

$$
[L f M]_{0}=[L f M]_{b}
$$

Two operating conditions which satisfy the equality in equation 27 should then provide the same degree of scattering. Consequently, attentuation of the discrete tone amplitude would be the same for two such cases.

A crude check of equation 27 can be made using the results shown in figure 64. Here the test conditions, corresponding to $M=0.1, f=15 \mathrm{kHz}$ and $M=0.3$, $\mathrm{f}=5 \mathrm{~Hz}$, satisfy equation 27 . The experimental measurements both show a $2.5 \mathrm{~dB}$ reduction in the tone amplitude. Equation 27 is also satisfied for the $M=0.2$, $\mathrm{f}=15 \mathrm{kHz}$ and $\mathrm{M}=0.3, \mathrm{f}=10 \mathrm{kHz}$ test conditions. Both spectra in this case show approximately a $3 \mathrm{~dB}$ reduction.

In figure 66 the shear layer thickness, $\delta$, at the acoustic ray cross-over point on the open jet lip line is about five times that of figure 64 assuming 
a constant spreading angle. The $M=0.1, f=5 \mathrm{kHz}$ case in figure 66 gives a $1 \mathrm{~dB}$ reduction in the tone while the $M=0.2 \mathrm{f}=15 \mathrm{kHz}$ case in figure 64 gives a $3 \mathrm{~dB}$ reduction. This comparison is not as good as the comparison in figure 64 but, further work would be needed to give a more definitive evaluation of equation 27. Also, future comparisons should be made using the $0.25 \mathrm{~Hz}$ analysis bandwidth instead of the $50 \mathrm{~Hz}$ bandwidth.

Equation 22 can also be used to derive an equation predicting the onset of turbulence scattering. A criterion must, however, first be set to determine the discrete tone amplitude attentuation for which scattering is considered to be important. Here the value of $R_{S}$ at which scattering is deemed significant will be taken to be 0.5 . Then assuming $\mathrm{L}=\mathrm{L}_{1}$ as above, and also that the root-mean-square turbulence velocity is $15 \%$ of the open jet velocity, equation 23 becomes

$$
\left(\frac{M L}{\lambda}\right)=0.5
$$

or

$$
\text { (f ML ) }=0.5 c_{0}
$$

Assuming a spreading half angle of $7^{\circ}$ for the shear layer, the shear layer thickness is approximately $0.3 \mathrm{~m}$ at the acoustic ray cross-over point for the $\mathrm{X} / \mathrm{R}_{\mathrm{O}}=$ 2.66 source position. Taking $c_{o}=355 \mathrm{~m} / \mathrm{sec}$ gives $\mathrm{f} M=558$; thus, for $\mathrm{M}=0.1$ scattering will become important at $\mathrm{f}=5580 \mathrm{~Hz}$. From figure $66 \mathrm{a}$ for $\mathrm{M}=0.1$ and $f=5000 \mathrm{~Hz}$ the peak of the tone is one $\mathrm{dB}$ below that for no scattering, indicating that there has been measureable scattering.

Equation 28 can be compared to the onset of turbulence scattering deduced experimentally in figure 53. Where scattering was shown to occur when $L / \lambda \geq 10$. For this condition, equation 28 gives $M=0.05$ as the. Mach number for which scattering becomes important. Figure 53 shows that for $\mathrm{L} / \lambda \approx 10 \mathrm{a} 1 \mathrm{~dB}$ attenuation in discrete tone amplitude occurs at $M=0.05$. This Mach number is equivalent to the value calculated from equation 28. The good comparison between theory and experiment verifies that equation 28 can be used to predict the onset for turbulence scattering.

The above result of Lighthill assumes single scattering. An alternate approach is to assume multiple scattering as in the analysis of Howe (ref. 25). Similar to the Lighthill model, Howe's approach assumes no mean flow, and because of this, gives no scattering in the frequency domain for the case of frozen turbulence. 
By assuming a Doppler shift due to the motion of the turbuience, the Howe theory has been modified by Amiet in reference 26 to account for a mean flow. Because the analysis is a high frequency theory and assumes multiple scattering it cannot be used for predicting the onset of significant scattering. Also, because of the multiple scattering assumption, the scattering is independent of frequency. Thus, in comparing the results of the theory and experiment, the experimental results for the highest frequency will be chosen since this best satisfies the multiple scattering assumption. To further satisfy this assumption, theory will only be compared with experiment at the farthest downstream source position corresponding to $\mathrm{X} / \mathrm{R}_{\mathrm{o}}=2.66$.

Using the $50 \mathrm{~Hz}$ spectrum analysis results in figure $66 \mathrm{c}$ the peak for the $M=0.3, f=15 \mathrm{kHz}$ case is expected to be $3 \mathrm{~dB}$ below the $\mathrm{M}=0.1$ case. The modified Howe theory gives a $10 \mathrm{~dB}$ difference in the peak level for these two cases. Thus, there is a significant difference between the theory and experiment. It is possible that this difference is due to the experiment not being in the applicable range of the theory; i.e., the theory assumes multiple scattering, whereas this assumption is not satisfied by the experiment.

In conclusion, additional work is needed on the multiple scattering theory in order to predict the experimental results. It is possible that a modification applied to the Howe theory will give a better prediction of the experimental results. 
CONCLUSIONS

A theoretical and experimental study was conducted to determine the effects of refraction and scattering on sound transmission through a circular open-jet shear layer. A generalized refraction theory was assessed experimentally for angle and amplitude changes across the shear layer. Both on-axis and off-axis source locations were investigated as frequency varied from 1 to $10 \mathrm{kHz}$ and freestream Mach number varied from 0.1 to 0.4 . Discrete tone scattering phenomena were investigated over the same Mach number range as frequency varied from $5 \mathrm{kHz}$ to $15 \mathrm{kHz}$. Attenuation of discrete tone amplitude and tone broadening were measured as a function of acoustic source position and radiation angle. The following conclusions are now available from this investigation.

\section{Refraction Effects for On-Axis Source}

a. Far-field noise directivity patterns measured in open-jet acoustic test facilities are significantly altered at test Mach numbers of 0.1 and greater due to. sound wave refraction by the open-jet shear layer. This is evident from measurements of the wavefront angle and amplitude changes associated with sound propagation through the shear layer.

b. For on-axis source locations, the refraction angle and amplitude changes for a circular cross-section open jet were well predicted by the zero thickness shear layer theory of Amiet over the Mach number range of 0.1 to 0.4 employed in the present experiment.

c. For the range of shear layer thicknesses considered in the present experiment, the refraction angle and amplitude changes were independent of shear layer thickness. This is evident from wavefront angle change measurements conducted at axial source positions between 0.6 and 4 jet radii downstream of the nozzle exit. Amplitude changes were also verified to be independent between 0.53 and 2.66 jet radii from the nozzle exit. This independence of shear layer thickness and shear layer divergence confirms a previous theoretical prediction.

d. The refraction angle and amplitude changes were independent of frequency over the $1 \mathrm{kHz}$ to $10 \mathrm{kHz}$ range considered in the present experiment. This independence confirms a previous theoretical prediction. Experimental verification of the amplitude independence using a discrete tone was complicated by the presence of sound scattering by the turbulent shear layer at high frequencies. 
a. The generalized off-axis refraction angle and amplitude correction theory developed in the present program predicts large differences between onaxis and off-axis corrections.

b. The off-axis angle and amplitude correction theory was confirmed experimentally for the case in which the observer and source were located in a plane passing through the open-jet axis and the source-to-shear layer distance was greater than one open jet-radius. Mach number dependence and frequency independence of the zero thickness shear layer refraction theory were validated.

c. For an off-axis source situated at a source-to-shear layer separation distance less than one jet radius, measurable differences between theory and experiment occurred for the refraction angle change. The disagreement, at present, is not understood. This result precluded conducting the amplitude correction study since placement of the far-field microphones requires knowledge of the angle change across the shear layer.

Scattering of Sound by Turbulence

a. Scattering is a combined function of the open jet absolute turbulence intensity (which scales with Mach number), acoustic source frequency, and propagation path length through the turbulent medium. Scattering effects were observed to increase as each of these individual parameters increased.

b. Several trends were observed in the experimental study. These were:

1) Scattering is stronger at angles close to the open jet axis than at $90^{\circ}$,

2) Scattering becomes stronger as the acoustic source position shifts downstream,

3) Scattering becomes significant as the ratio of shear layer propagation path length to acoustic wave length approaches a value of 10 .

c. Reduction of the discrete tone amplitude on the refracted ray path is caused by direction scattering of sound to new angles. Tone broadening of the spectrum measured on the refracted ray path is caused by Doppler-shifting of sound waves by turbulent regions upstream and downstream of the acoustic ray shear layer cross-over point. The existence of tone broadening (or frequency scattering) implies the occurrence of direction scattering.

d. The frequency at which the onset of turbulence scattering occurs can be estimated using an analysis developed during the present study. 


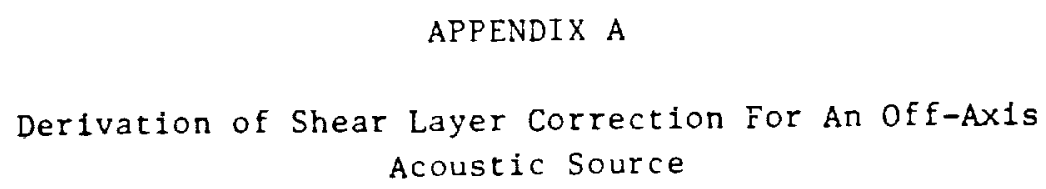

Figures 21 and 22 show the basic geometry and the coordinate system used in the following derivation. These figures were previously described in the section entitled "Theoretical Formulation of the Refraction Problem."

Refraction Angle Change - The angular change of a ray on passing through the shear layer is determined by treating the waves on either side of the shear layer as plane waves and matching phase across the shear layer. This planar assumption should be valid as long as the wavelength is smaller than the open jet radius.

The small disturbance pressure field produced by an arbitrary source can be written as a Fourier composition of plane waves of the form

$$
P_{i}=e^{-i\left(\theta-k_{21} z\right)}
$$

where

$$
\Theta \equiv-\omega \uparrow+k_{x} x+k_{y} y
$$

and $k$ and $k$ represent wavevectors in the $x, y, z$ coordinate system. Substitution of equation (A.1) in the wave equation

$$
\begin{gathered}
\left(\frac{D^{2}}{D t^{2}}-C_{0}^{2} \nabla^{2}\right) P=0 \\
\frac{D}{D \dagger} \equiv \frac{\partial}{\partial t}+U \frac{\partial}{\partial x}
\end{gathered}
$$

gives

$$
\bar{k}_{z 1}=\left(1-M \bar{k}_{x}\right)^{2}-\bar{k}_{x}^{2}-\bar{k}_{y}^{2}
$$

where the overbar on $k$ indicates normalization by $\omega / c_{0} ; 1 . e ., k=k c_{0} / \omega$.

The normal to a wavefront is found by taking the gradient of the phase of equation (A.1) giving

$$
\hat{n}_{e}=\frac{\bar{k}_{x} \hat{i}+\bar{k}_{y} \hat{j}+\bar{k}_{z 1} \hat{k}}{1-M \bar{k}_{x}}
$$


Since the wavefronts produced by a source in a stream are spheres with their center at the retarded source position, this normal vector points from the retarded source position to $x_{1}, y_{1}{ }^{\prime}, z_{1}{ }^{\prime}$. The direction of energy propagation (the line connecting the present source position to $x_{1}, y_{1}^{\prime}, z_{1}^{\prime}$ ) can be found by adding $\hat{i} M$ to equation (A.5). Thus.

$$
\hat{n}_{1}=\frac{\hat{n}_{e}+\hat{i} M}{\left|\hat{n}_{e}+\hat{i} M\right|}=\frac{\hat{i}\left(M+\beta^{2} \bar{k}_{x}\right)+\hat{j} k_{y}+\hat{k} \bar{k}_{z 1}}{\sqrt{1+M^{2}\left[\left(1-M \bar{k}_{x}\right)^{2}-\bar{k}_{x}^{2}\right]}}
$$

The transmitted wave at point $x_{1}, y_{1}{ }^{\prime}, z_{1}{ }^{\prime}$ can be written

$$
P_{1}=T e^{-1\left(\Theta+k_{22} z\right)}
$$

Substitution in equation (A. 3 ) but with $M=0$ gives

$$
\bar{k}_{z 2}^{2}=1-\bar{k}_{x}^{2}-\bar{k}_{y}^{2}
$$

Taking the gradient of the phase of equation (A.7) gives for both the normal to a wavefront above the shear layer and the direction along which energy is propagated

$$
\hat{n}_{z}=\bar{k}_{x} \hat{i}+\bar{k}_{y} \hat{j}+\bar{k}_{z 2} \hat{k}
$$

Equations (A.6) and (A.9) give the relation between the ray paths for the incident and transmitted rays. Rather than have this relation in terms of the parameters $k_{x}$ and $k_{y}$ (which must be equal in equations (A. $\theta$ ) and (A.9) in order for the phase of the incident and transmitted waves to be equal across the shear layer) it is more useful to put the relation in terms of the angles $\theta$ and $\phi$. Then, $x_{1}, y_{1}$ and $z_{1}$ can be written as

$$
\begin{aligned}
& x_{1}=r_{1} \cos \theta_{c} \\
& y_{1}=r_{1} \sin \theta_{c} \cos \gamma_{c} \\
& z_{1}=r_{1} \sin \theta_{c} \sin \gamma_{c}
\end{aligned}
$$

with

$$
r_{1}=\frac{0 \sin \alpha}{\cos \phi_{c} \sin \theta_{c}}=\frac{a\left(\sin \gamma_{c}-g \sin \phi_{c}\right)}{\sin \theta_{c}}
$$


and

$$
\begin{gathered}
\gamma \equiv \phi+\alpha \\
g \equiv 1-h / o
\end{gathered}
$$

The first equality in equation (A.11) can be verified from figure 14, and the second equality is found from equation (A.20), to be derived later. The normal $\hat{n}_{1}$
along $r_{1}$ is then

$$
\hat{n}_{1}=\hat{i} \cos \theta_{c}+\hat{j} \sin \theta_{c} \cos \gamma_{c}+\hat{k} \sin \theta_{c} \sin \gamma_{c}
$$

Comparisons of equations (A.6) and (A.12) show that

$$
\cos \theta_{c}=\frac{M+\beta^{2} \bar{k}_{x}}{\sqrt{1+M^{2}\left[\left(1-M \bar{k}_{x}\right)^{2}-\bar{k}_{x}^{2}\right]}}
$$

or

$$
\bar{k}_{x}=\frac{1}{\beta^{2}}\left[\frac{\cos \theta_{c}}{\sqrt{1-M^{2} \sin ^{2} \theta_{c}}}-M\right]
$$

Also

$$
\begin{aligned}
& \bar{k}_{y}=\frac{\sin \theta_{c} \cos \gamma_{c}}{\sqrt{1-M^{2} \sin ^{2} \theta_{c}}} \\
& \bar{k}_{21}=\frac{\sin \theta_{c} \sin \gamma_{c}}{\sqrt{1-M^{2} \sin ^{2} \theta_{c}}}
\end{aligned}
$$

For the transmitted wave the normal $\hat{n}_{2}$ along $r_{2}$ can be written

$$
\hat{n}_{2}=\hat{i} \cos \theta_{1}+\hat{j} \sin \theta_{1} \cos \gamma_{1}+\hat{k} \sin \theta_{1} \sin \gamma_{1}
$$

Comparison with equation (A.9) gives

$$
\bar{k}_{x}=\cos \theta_{1}
$$




$$
\begin{aligned}
& \bar{k}_{y}=\sin \theta_{1} \cos \gamma_{t} \\
& \bar{k}_{z 2}=\sin \theta_{1} \sin \gamma_{1}
\end{aligned}
$$

By equating the values of $k_{x}$ and $k_{y}$ given by equations (A.13) and (A.15), the incident and transmitted angles are found to be

$$
\begin{aligned}
& \beta^{2} \cos \theta_{1}=\frac{\cos \theta_{c}}{\sqrt{1-M^{2} \sin ^{2} \theta_{c}}}-M \\
& \sin \theta_{1} \cos \gamma_{1}=\frac{\sin \theta_{c} \cos \gamma_{c}}{\sqrt{1-M^{2} \sin ^{2} \theta_{c}}}
\end{aligned}
$$

Equation (A.16) can also be written

$$
\tan \theta_{c}=\frac{\zeta_{1}}{\beta^{2} \cos \theta_{1}+M}
$$

where

$$
\zeta_{1}^{2}=\left(1-M \cos \theta_{1}\right)^{2}-\cos ^{2} \theta_{1}
$$

which agrees with equation 1 of reference 6 . Other useful relations are

$$
\begin{aligned}
\sin \theta_{c} & =\frac{\zeta_{1}}{\sqrt{1+M^{2} \zeta_{1}^{2}}} \\
\zeta_{1} & =\frac{\sin \theta_{c}}{\sqrt{1-M^{2} \sin ^{2} \theta_{c}}}
\end{aligned}
$$

The angle a can be related to $\phi_{c}$ by applying the sine rule to triangle $S A B$ in figure 22. Thus, 


$$
\cos \gamma_{c}=g \cos \phi_{c}
$$

Using equation (A.19), equation (A.17) can be rewritten

$$
\frac{1}{\zeta_{1}} \sin \theta_{1} \cos \gamma_{1}=\cos \gamma_{c}
$$

The above relations determine completely the angle change needed in the shear layer correction.

Refraction Amplitude Correction - The amplitude correction will now be determined by calculating the ray tube divergence. Consider a ray tube beneath the shear layer formed by varying $\theta_{c}$ and $\phi_{c}$ by amounts $d \theta_{c}$ and $d \phi_{c}$, respectively. This produces a ray tube with cross-sectional-area at the shear layer

$$
d A_{1}=r_{1}^{2} \sin \theta_{c} d \theta_{c} d \phi_{c}
$$

Above the shear layer the angle variations are

$$
\begin{gathered}
d \theta_{1}=\frac{\partial \theta_{1}}{\partial \theta_{c}} d \theta_{c} \\
d \phi_{1}=\frac{\partial \phi_{1}}{\partial \theta_{c}} d \theta_{c}+\frac{\partial \phi_{1}}{\partial \phi_{c}} d \phi_{c}
\end{gathered}
$$

Note from equation (A.16) that $\partial \theta_{t} / \partial \phi_{c}=0$ since $\varepsilon_{c}$ and $\phi_{c}$ are independent variables. Therefore, this term does not appear in equation (A.23a). The partial derivatives $c$ an be found from equations (A.16) and (A.21) to be

$$
\begin{gathered}
\frac{\partial \theta_{1}}{\partial \theta_{c}}=\frac{\zeta_{1}^{3}}{\sin \theta_{1} \sin ^{2} \theta_{c}} \\
\frac{\partial \phi_{1}}{\partial \phi_{c}}=\frac{g \zeta_{1} \sin \phi_{c}}{\sin \gamma_{1} \sin \theta_{1}}-\frac{\partial \alpha}{\partial \phi_{c}}
\end{gathered}
$$




$$
\begin{gathered}
\frac{\partial a}{\partial \phi_{c}}=9 \frac{\sin \phi_{c}}{\sin \gamma_{c}}-1 \\
\frac{\partial \phi_{1}}{\partial \theta_{c}}=\frac{\cos \gamma_{1} \cot \theta_{1} \sin \theta_{c}-\cos \gamma_{c} \cos \theta_{c}}{\sin \gamma_{i} \sin \theta_{1}\left(1-M^{2} \sin ^{2} \theta_{c}\right)^{3 / 2}}
\end{gathered}
$$

In order to find the area which the ray tube intercepts on the tangent plane, note that $z_{1}$ will remain constant in equation (A.10) as $\theta_{C}$ and $\phi_{c}$ are
varied. From equation (A.10)

$$
\begin{aligned}
& \frac{x_{1}}{z_{1}}=\frac{\cot \theta_{c}}{\sin \gamma_{c}} \\
& \frac{y_{1}}{z_{1}}=\cot \gamma_{c}
\end{aligned}
$$

Thus,

$$
\begin{gathered}
d x_{1}=-r_{1}\left[\frac{d \theta_{c}}{\sin \theta_{c}}+\cos \theta_{c} \cot \gamma_{c} d \phi_{c}\right] \\
d y_{1}=-r_{1} \frac{\sin \theta_{c}}{\sin \gamma_{c}} d \phi_{c}
\end{gathered}
$$

A variation $d \theta_{c}$ gives a contribution to $d x_{1}$, while a subsequent variation $d^{*} c$ gives both ${ }^{c} d_{d x}$ and $d y$ contribution. 'This indicates that the ray tube area intercepted by the tangent plane is a parallelogram; its area $\mathrm{dA}_{2}$ is the $d \theta_{c}$ contribution to $\mathrm{dx}_{1}$ times the $\mathrm{d}_{\mathrm{c}}$ contribution to $\mathrm{dy}_{1}$, or

$$
d A_{2}=\frac{r_{1}^{2} d \phi_{c} d \theta_{c}}{\sin \gamma_{c}}=\frac{d A_{1}}{\sin \gamma_{c} \sin \theta_{c}}
$$

The cross-sectional area $\mathrm{dA}_{3}$ just above the shear layer is then, by a sinilar argument

$$
d A_{3}=d A_{2} \sin \gamma_{1} \sin \theta_{1}
$$


Equation (A.30) can also be derived by setting $r_{2}=0$ in equations (A.34) to be derived later.

The rate of ray tube divergence changes on passing through the shear layer, and the ray tube cross-sectional area must now be calculated for a point at an arbitrary distance above the shear layer. The equations for a point $x_{2}, y_{2}, z_{2}$ are

$$
\begin{aligned}
& x_{2}=x_{1}+r_{2} \cos \theta_{\uparrow}=r \cos \theta_{m} \\
& y_{2}=y_{1}+r_{2} \sin \theta_{1} \cos \gamma_{\uparrow} \\
& z_{2}=z_{1}+r_{2} \sin \theta_{1} \sin \gamma_{1}
\end{aligned}
$$

with $r^{2}=x_{2}^{2}+y_{2}^{2}+z_{2}^{2}$

By varying first $\theta_{c}$ and then $\phi_{c}$, the point $x_{2}, y_{2}, z_{2}$ will trace out two sides of an area. By taking the cross product of ${ }^{2}$ the vectors defining these two sides and then taking the dot product with the unit vector $\hat{n}_{2}$ given by equation (A.14), the ray tube cross-sectional area will be determined. In taking the derivatives of equation (A.31), $r_{2}$ will be taken as constant, as the variation of $r_{2}$ with $\theta_{c}$ and $\phi_{c}$ is irrelevant in the final area result. Thus,

$$
\begin{gathered}
\frac{\partial x_{2}}{\partial \theta_{c}}=\frac{-r_{1}}{\sin \theta_{c}}-r_{2} \sin \theta_{1} \frac{\partial \theta_{1}}{\partial \theta_{c}} \\
\frac{\partial y_{2}}{\partial \theta_{c}}=r_{2}\left(\cos \theta_{1} \cos \gamma_{1} \frac{\partial \theta_{1}}{\partial \theta_{c}}-\sin \theta_{1} \sin \gamma_{1} \frac{\partial \phi_{1}}{\partial \theta_{c}}\right) \\
\frac{\partial z_{2}}{\partial \theta_{c}}=r_{2}\left(\cos \theta_{1} \sin \gamma_{1} \frac{\partial \theta_{1}}{\partial \theta_{c}}+\sin \theta_{1} \cos \gamma_{1} \frac{\partial \phi_{1}}{\partial \theta_{c}}\right)
\end{gathered}
$$



where $z_{1}$ is constant and the variation of $x_{1}$ and $y_{1}$ with $\theta_{c}$ was found using
equation (A.28;. Also,

$$
\begin{aligned}
& \frac{\partial x_{2}}{\partial \phi_{c}}=-r_{1} \cos \theta_{c} \cot \gamma_{c} \\
& \frac{\partial y_{2}}{\partial \phi_{c}}=-r_{1} \frac{\sin \theta_{c}}{\sin \gamma_{c}}-r_{2} \sin \theta_{\uparrow} \sin \gamma_{1} \frac{\partial \phi_{1}}{\partial \phi_{c}} \\
& \frac{\partial z_{2}}{\partial \phi_{c}}=r_{2} \sin \theta_{1} \cos \gamma_{1} \frac{\partial \phi_{1}}{\partial \phi_{c}}
\end{aligned}
$$

The cross-sectional area $\mathrm{dA}_{4}$ is then

$$
\begin{aligned}
& \frac{d \varepsilon_{1}}{d}=\left(\hat{i} \frac{\partial x_{2}}{\partial \theta_{c}}+\hat{j} \frac{\partial y_{2}}{\partial \theta_{c}}+\hat{k} \frac{\partial z_{2}}{\partial \theta_{c}}\right) d \theta_{c} \\
& \frac{d \varepsilon_{2}}{d}=\left(\hat{i} \frac{\partial x_{2}}{\partial \phi_{c}}+\hat{\jmath} \frac{\partial y_{2}}{\partial \phi_{c}}+\hat{k} \frac{\partial z_{2}}{\partial \phi_{c}}\right) d \phi_{c} \\
& d \Delta_{4}=\hat{n}_{2} \cdot\left(\underline{d f_{1}} \times \underline{d \varepsilon_{2}}\right)
\end{aligned}
$$

Thus,

$$
\begin{aligned}
\frac{d A_{4}}{d A_{3}}= & \left(1+\frac{r_{2}}{r_{1}} \frac{\sin \theta_{c}}{\sin \theta_{1}} \frac{\partial \theta_{1}}{\partial \theta_{c}}\right)\left(1+\frac{r_{2}}{r_{1}} \frac{\sin \gamma_{c}}{\sin \gamma_{1}} \frac{\sin \theta_{1}}{\sin \theta_{c}} \frac{\partial \phi_{1}}{\partial \phi_{c}}\right) \\
& +\frac{r_{2}}{r_{1}}\left(\frac{\sin \theta_{c}}{\zeta_{1}}\right)^{3}\left(\sin \theta_{1} \frac{\partial \phi_{1}}{\partial \theta_{c}}\right)^{2}
\end{aligned}
$$


Equation (A.35) gives the ray tube divergence behavior above the shear layer. This equation allows one to calculate the sound level just outside the shear layer in terms of the far-field sound.

The incident pressure just inside the shear layer is now needed, and will be calculated from the shear layer transmission coefficient. In crossing the shear layer, the wavefront can be treated locally as being plane. Then the velocity potentials for the incident, reflected and transmitted waves can be written

$$
\begin{aligned}
& \Phi_{i}=e^{-i\left(\Theta+k_{z 1} z\right)} \\
& \Phi_{r}=R e^{-i\left(\Theta-k_{z 1} z\right)} \\
& \Phi_{1}=T_{\Phi} e^{-i\left(\Theta+k_{22} z\right)}
\end{aligned}
$$

where $\theta$ is given by equation (A.2), $k_{z_{1}}$ by equation (A.4) and $k_{z_{2}}$ by equation (A.8).

The pressure and fluid displacement will be matched across the shear layer. The pressure is related to the velocity potential by

$$
P=-\rho_{0} \frac{D \Phi}{D t}
$$

Matching the pressure across the shear layer at $z_{1}$ gives

$$
\left(1-M \bar{k}_{x}\right)\left[e^{-i z_{1} k_{z} \mid}+R e^{i z_{1} k_{z 1}}\right]=T_{\Phi} e^{-\mid 2, k} z z
$$

To match the fluid displacement across the shear layer, the interface can be considered to be rippled by the acoustic waves, the ripple moving in the $x$ direction with velocity $k_{x} / \mathrm{u}$. If the observer moves in the $x$ direction with the ripple, the mean flow velocity outside the shear layer will be $-w / k_{x}$ while that inside will be $U_{0}-w / k_{x}$. Denoting the perturbation $z$ velocity by $w$ and equating flow slopes gives

$$
w_{1}=\frac{w_{1}+w_{r}}{1-M \bar{k}_{x}}
$$



becomes

Then, using the fact that $w=\partial \Phi / \partial z$ and using equations (A.36), equation (A. 38)

$$
T_{\Phi} k_{22}\left(1-M \bar{k}_{x}\right) e^{-i z_{1} k_{22}}=k_{21}\left(e^{-i z, k_{21}}-R e^{i z_{1} k_{21}}\right)
$$

Combining equations (A.38) and (A.40),

$$
T_{\Phi}\left[1+\left(1-M \bar{k}_{x}\right)^{2} k_{z 2} / k_{z 1}\right]=2\left(1-M \bar{k}_{x}\right) e^{i z_{1}\left(k_{z 2}-k_{z 1}\right)}
$$

$I_{\Phi}$ is the transmission coefficient for the potential. The transmission coefficient for pressure is found by noting from equation (A.37) that

$$
\left|T_{D}\right|=\left|\frac{P_{i}}{P_{i}}\right|=\left|\frac{\Phi_{1}}{\left(1-M \bar{k}_{x}\right) \Phi_{i}}\right|=\frac{\left|T_{\phi}\right|}{1-M \bar{k}_{x}}
$$

Thus,

$$
\left|T_{P}\right|=\frac{2}{1+\left(1-M \bar{k}_{x}\right)^{2} k_{22} / k_{21}}
$$

From equations (A.13C), (A.15C), and (A.19)

$$
\frac{k_{22}}{k_{21}}=\frac{\sin \theta_{1} \sin \gamma_{1}}{\zeta_{1} \sin \gamma_{c}}
$$

The final result for the amplitude correction can now be given. Equation (A.35) gives the ratio of the square of the pressure just outside the shear layer to that at the observer. Equations (A.43) and (A.44) give the ratio of pressure just outside to the incident pressure just inside the shear layer. Thus, the Incident pressure just inside the shear layer can be calculated from that measured at the observer position. By extrapolating this calculated pressure by the factor $r_{1} / r$, the corrected pressure at a distance $r$ from the source can be calculated. The final result for the correction factor is then 


$$
\begin{aligned}
\left|\frac{P_{c}}{P_{m}}\right|= & \left\{\left(\frac{r_{1}}{r}+\frac{r_{2}}{r} \frac{\zeta_{1}^{3}}{\sin ^{2} \theta_{1} \sin \theta_{c}}\right)\left(\frac{r_{1}}{r}+\frac{r_{2}}{r} \frac{\sin \gamma_{c}}{\sin \gamma_{1}} \frac{\sin \theta_{t}}{\sin \theta_{c}} \frac{\partial \phi_{1}}{\partial \phi_{c}}\right)\right. \\
& \left.\left.+\frac{r_{1} r_{2}}{r^{2}}\left(\frac{\sin \theta_{c}}{\zeta_{1}}\right)^{3}\left(\sin \theta_{1} \frac{\partial \phi_{1}}{\partial \theta_{c}}\right)^{2}\right]^{1 / 2} \frac{\sin \theta_{c}}{\zeta_{1}^{3 / 2}} \sqrt{\frac{\sin \theta_{1} \sin \gamma_{t}}{\sin \gamma_{c} \partial \phi_{1} / \partial \theta_{c}}}\right\} \\
& \left\{\frac{\zeta_{1}^{3 / 2}}{2 \sin \theta_{c}} \sqrt{\frac{\sin \gamma_{c} \partial \phi_{1} / \partial \theta_{c}}{\sin \theta_{1} \sin \gamma_{t}}}\left[1+\left(1-M \cos \theta_{1}\right)^{2} \frac{\sin \theta_{1} \sin \gamma_{1}}{\zeta_{1} \sin \gamma_{c}}\right]\right)
\end{aligned}
$$

where $\frac{\partial \phi_{t}}{\partial \phi_{C}}$ and $\frac{\partial \phi_{t}}{\partial \phi_{c}}$ are given by equations (A.25a) and (A.26), respectively. 


\section{Listing of Computer Program for Generalized Refraction Theory}

The computer program listed on the following pages solves the angle and amplitude corrections for a sound source off axis inside a cylindrical zero thickness shear layer. This program gives the results of Eqs. (A.16) and (A.17) for the angle correction and $\mathrm{Eq}$. (A.45) for the amplitude correction with relevant parameters in these equations given by Eqs. (A11), (A18), A(25a), A(26) and A(31). The necessary inputs to the program are

$$
\begin{aligned}
\mathrm{A} & =\text { shear layer } \text { radius }=\mathbf{a} \\
\mathrm{G} & =1-\mathrm{h} / \mathrm{a} \\
\mathrm{Z} & =\text { sideline or constant radius distance } \\
\mathrm{M} & =\text { tunnel Mach number } \\
\mathrm{PHC}=\phi_{\mathrm{C}} & =\text { one of the two corrected angles }
\end{aligned}
$$

The angles and various geometric parameters are defined in Figs. 21 and 22 . This program does not give an explicit solution in that the measured observer angles are not specified as input, but are output. A program does exist at UTRC which allows input of the measured observer angles, but this requires iteration. The observer distance from the source is an input to the above program, however. The angle $\phi_{c}$ is $90^{\circ}$ for the case with the source, the observer and the tunnel axis in the same plane. For the case $\phi_{c}=90^{\circ}$, the parameter $\mathrm{g}$ is 1 for the case where the source is just inside the near shear layer, while $g=-1$ represents the case where the source is just inside the shear layer on the far side of the tunnel. For a source on the tunnel axis $g=0$. Also, the case $\left(g=-g_{0}, \phi_{c o}\right)$ is the same as
$\left(g=g_{0}, \phi_{C}=-\phi_{c o}\right)$ for any values $g_{0}$ and $\phi_{c o}$

The outputs of the program are

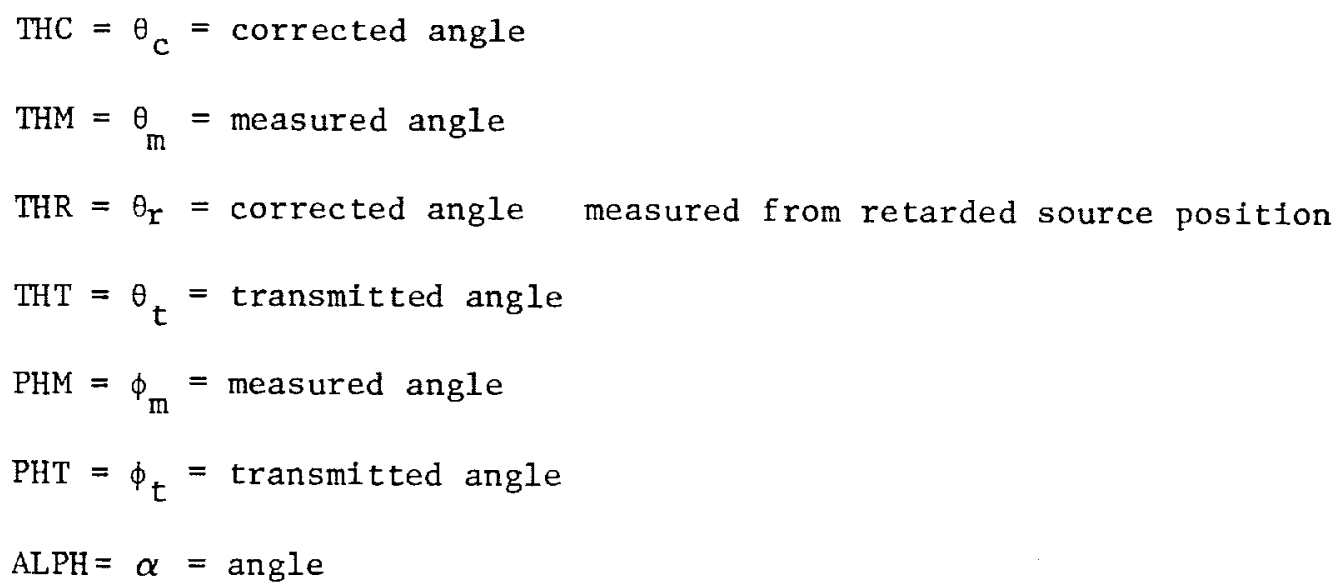




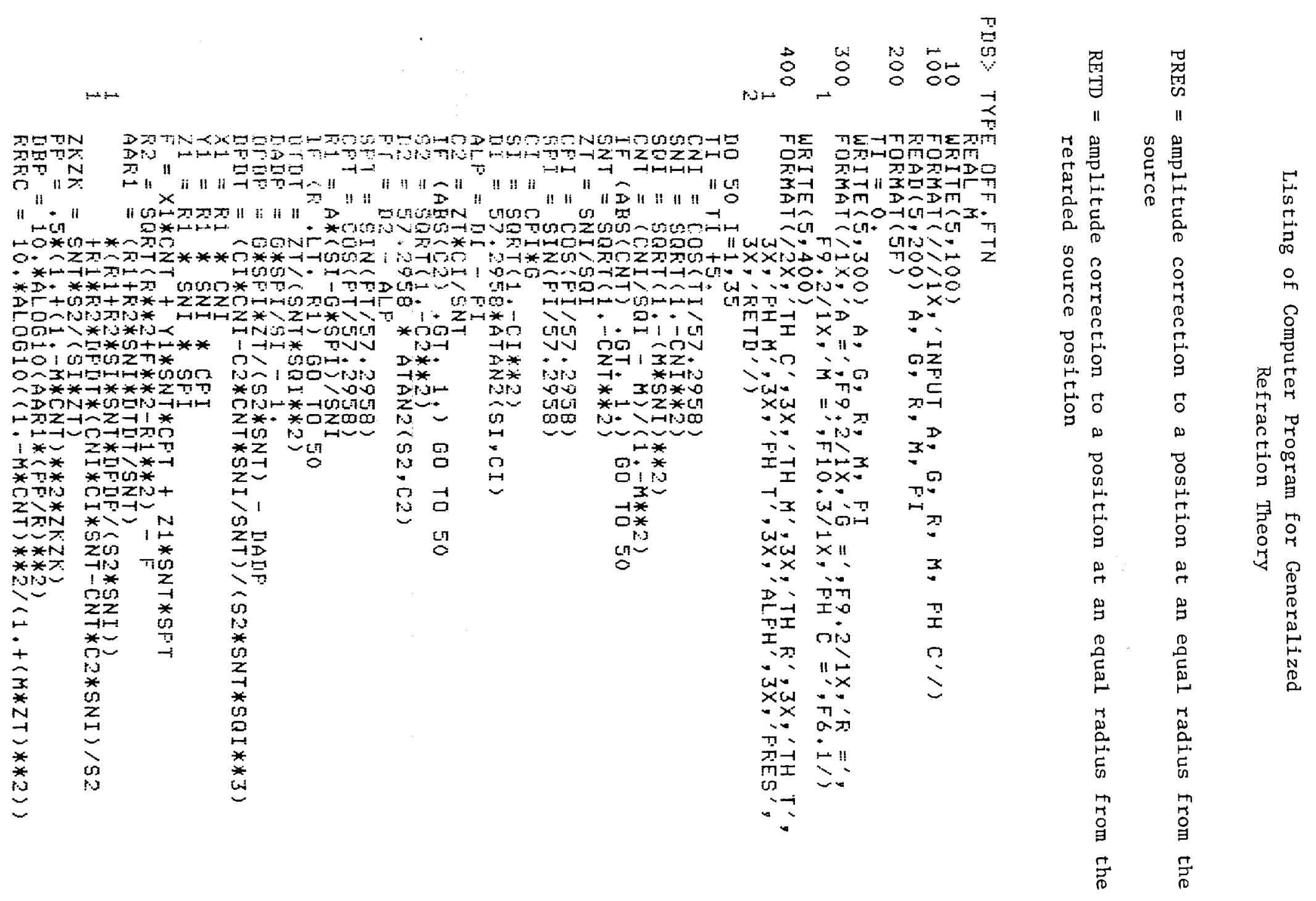




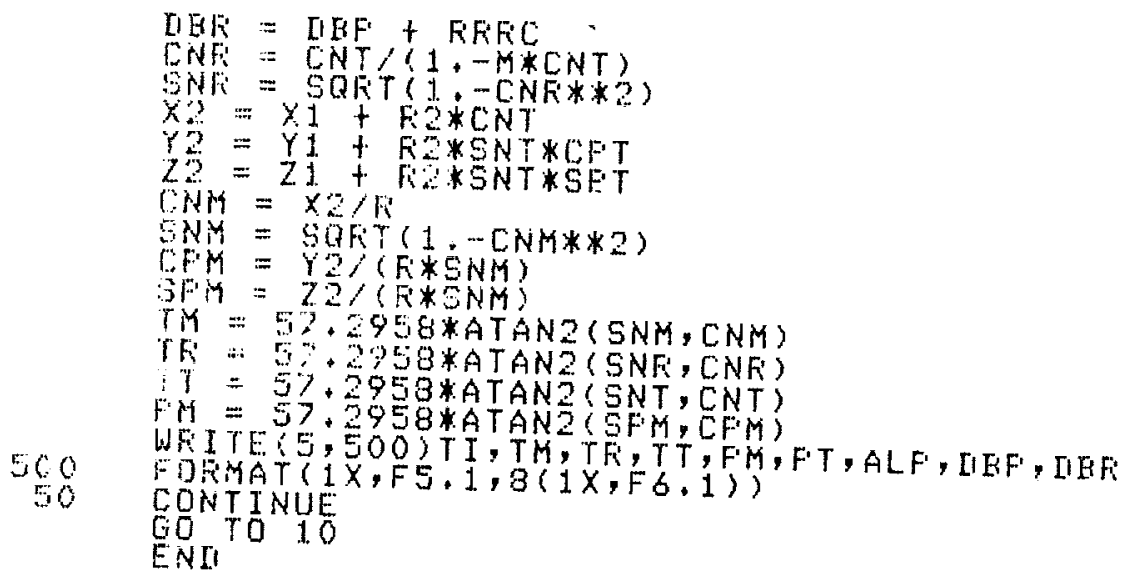

A sample printout is given below for a sideline microphone geometry with $M=0.3$. The predictions correspond to an on-axis acoustic source and far-field microphone position situated in a plane coinciding with the jet axis. Under these conditions $\phi_{\mathrm{m}}=90^{\circ}$ and $\phi_{t}=90^{\circ}$ (not included in printout) and $\alpha=0^{\circ}$. The parameter RETD is not included in the printout. The resulting table of $\theta_{c}$ versus $\theta_{m}$ and $\theta_{m}$ (or $\theta_{c}$ ) versus PRESS was used to plot the angle corrections and amplitude corrections in figures 28 and 30 . Angles are given in degrees while the amplitude corrections, PRESS, are given in decibels. The input distances, $A$ and $Z$, can be entered in any system of units. The example presented here uses the English system of units with the shear layer radius, A, and the observer distance, $\mathrm{Z}$, given in inches. 
I NPUT A, G, Z, M, PH C $18,0,72, .3,90$
$A=18.90$
$G=0.00$
$z=72.00$
$M=0.300$
PH C $=90.0$

TH C TH M TH R TH T PH M

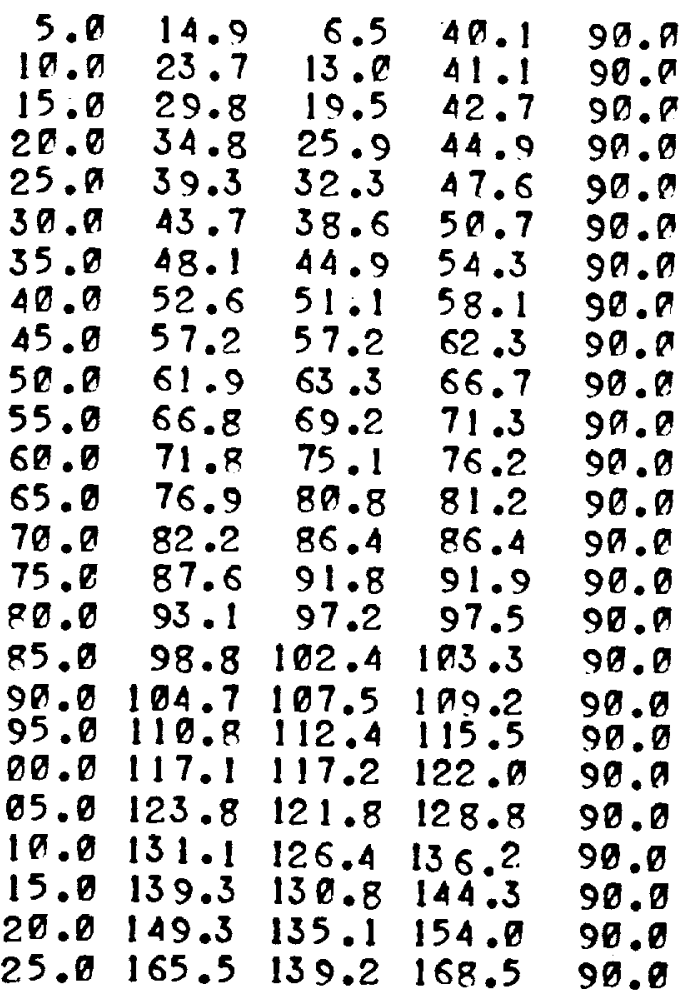


According to equation( 5 ) derived earlier

$$
\Delta_{T}^{\prime}-\Delta_{T}=\Delta^{\prime}-\Delta=\frac{f l}{C_{0}}\left[\sin \left(\mu_{0}+\mu_{1}\right)-\sin \mu_{0}\right]
$$

Using the trigonometric identity for the sum of two angles and expanding in a Fourier series gives

$$
\begin{aligned}
\Delta_{T}^{\prime}-\Delta_{T}=\frac{f l}{c_{0}} & {\left[\left(\mu_{0}+\frac{\mu_{0}^{3}}{3}+\cdots\right) \cos \mu_{1}+\left(1-\frac{\mu_{0}^{2}}{2}+\ldots\right) \sin \mu_{1}\right.} \\
& \left.-\left(\mu_{0}+\frac{\mu_{0}^{3}}{3}+\ldots\right)\right]
\end{aligned}
$$

Since $\mu_{0}$ was consistently held at values less then 0.016 radians, higher order terms may be neglected leaving

$$
\begin{array}{r}
\Delta_{T}^{\prime}-\Delta_{T}=\frac{f \ell}{c_{0}} \sin \mu_{1}\left[1+\frac{\mu_{0}\left(\cos \mu_{1}-1\right)}{\sin \mu_{1}}\right] \\
\text { or } \Delta_{T}^{\prime}-\Delta_{T}=\frac{f x}{c_{0}} \sin \mu_{1}\left[1-\frac{2 \mu_{0} \sin ^{2}\left(\mu_{1} / 2\right)}{\sin \mu_{1}}\right]
\end{array}
$$

The maximum.contribution from the second term in equation (B.3) occurs when $\mu_{1}=\pi / 2$. Then

$$
\Delta_{T}^{\prime}-\Delta_{T}=\frac{f k}{C_{0}}\left[1-\mu_{0}\right]
$$

Here $\mu_{0}$ provides a negligible contribution to the phase difference. Hence, an adequate approximation for the phase difference is

$$
\Delta_{T}^{\prime}-\Delta_{T}=\frac{f \ell}{C_{0}} \sin \mu_{1}
$$

This expression is valid to within .016 cycles. Finally,

$$
\mu_{1}=\sin ^{-1}\left[\frac{c_{0}\left(\Delta_{T}^{\prime}-\Delta_{I}\right)}{f \ell}\right]
$$


Figure 72 depicts the ray path as the acoustic wave is transmitted through the shear layer. The point, $x_{0}$, at which the sound emerges from the airstream is given by

$$
x_{0}=x_{2}-x_{3}
$$

But

$$
x_{2}=r \sin \left(90-\theta_{m}\right)
$$

and

$$
x_{3}=(Y-h) \tan \left(90-\theta_{m}-\mu_{1}\right)
$$

Thus

$$
x_{0}=r \sin \left(90-\theta_{m}\right)+(y-h) \tan \left(\mu_{1}-90+\theta_{m}\right)
$$

or

$$
x_{0}=r \cos \theta_{m}-\left(r \sin \theta_{m}-h\right) \cot \left(\mu_{1}+\theta_{m}\right)
$$

Finally the original propagation angle inside the flow is given by the expression

$$
\theta_{c}=\tan ^{-1}\left(\frac{h}{x_{0}}\right)
$$


Facility background noise contributed significantly to the two microphone cross-correlation function at Mach numbers greater than 0.1 . This effect can be demonstrated analytically. The net pressure, $P$, sensed by microphone $m_{2}$ in figure 73 is

$$
P_{2}(t)=B_{2} \cos \left[\omega t-2 \pi\left(\Lambda+\Delta_{2}\right)\right]+P_{2}^{\prime}(t)
$$

Here $B_{2}$ represents the frequency dependent discrete tone amplitude while $\mathrm{P}_{2}$ denotes the facility random background noise. The time dependence is expressed relative to the signal generator which drives the acoustic source in figure 73 The parameter $\Lambda$ represents the total phase lag between the signal generator output and the microphone input. Here

$$
\Lambda=\Delta_{0}+\Delta_{s}+\Delta_{p}
$$

where $\Delta_{p}$ is the propagation phase delay between the speaker and the microphone. A similar expression exists for the signal at $m_{1}$ except that the pure tone is out of phase by $\Delta^{\prime}$ relative to $m_{2}$ :

$$
P_{1}(t)=B_{1} \cos \left[\omega t-2 \pi\left(\Lambda+\Delta^{\prime}+\Delta_{1}\right)\right]+P_{1}^{\prime}(t)
$$

The resulting cross-correlation function $S$, is then

$$
S_{1,2}(\tau)=\left\langle P_{1}(t) P_{2}(t)\right\rangle
$$

or

$$
\begin{aligned}
S_{1,2}(\tau)=B_{1} B_{2}\langle & \cos \left[\omega t-2 \pi\left(\Lambda+\Delta^{\prime}+\Delta_{1}\right)\right] . \\
& \left.\cos \left[\omega t-2 \pi\left(\Lambda+\Delta_{2}\right)\right]\right\rangle \\
& +\left\langle P_{1}^{\prime}(t+\tau) P_{2}^{\prime}(t)\right\rangle
\end{aligned}
$$

The second term in equation (D.5) corresponds to the random noise contribution which dominates the correlation trace in figure 34 . 
An alternate approach for extracting only the periodic component is to separately cross-correlate each microphone signal with the acoustic driver signal. This technique, described by Schlinker in reference 23, was applied to the measurement of standing waves in a duct using flush mounted wall microphones. The microphones sensed both the acoustic pressure fluctuations and the random turbulent boundary layer pressure fluctuations.

The method is lllustrated schematically in figure 73 . The output voltage, $v_{2}$, from $m_{2}$ is cross-correlated with the signal generator voltage, $v_{S}(t)=v_{0}$ cosut, resulting in

$$
S_{2,5}(\tau)=G_{2} V_{0} B_{2}\left\langle\cos \left[\omega^{\dagger}-2 \pi\left(\Lambda+\Delta_{2}\right)\right] \cos \left[\omega^{\dagger}+\tau\right]\right\rangle
$$

Since the cross-correlation between the periodic driver signal and the random background noise is zero, this term is not included in equation D.6a. Note that the microphone system sensitivity, $G_{2}$, has been used to link the acoustic pressure and the voltage output. Replacing the trigonometric terms by the complex notation for the cosine function gives

$$
\begin{array}{r}
S_{2, S}(\tau)=\frac{1}{4} G_{2} B_{2} V_{0}\left\langle e^{2 i \omega t} e^{i\left(\omega \tau-\phi^{*}\right)}+e^{-2 i \omega t} e^{-i\left(\omega t-\phi^{*}\right)}\right. \\
+e^{i\left(\omega \tau+\phi^{*}\right)}+e^{\left.-i\left(\omega \tau+\phi^{*}\right)\right\rangle}
\end{array}
$$

where $\phi^{*}=2 \pi\left(\Lambda+\Delta_{2}\right)$. The time average of the first two periodic terms in equation D.6b is zero. Hence,

$$
S_{2,5}(\tau)=\frac{1}{2} G_{2} B_{2} V_{0} \cos \left[\omega \tau-2 \pi\left(\Lambda+\Delta_{2}\right)\right]
$$

The measured cross-correlation shown in figure 36 can be represented by a peak voltage amplitude $\mathrm{H}_{2}$ and a phase between $\mathrm{V}_{\mathrm{s}}$ and $\mathrm{P}_{2}$

$$
S_{2, s}(\tau)=H_{2} \cos \left[\omega \tau-2 \pi\left(n_{2}+\eta_{2}\right)\right]
$$

where $n$ is an integer. 
Comparing like terms in equations (D.7) and (D.8) provides

$$
\eta_{2}=\Lambda+\Delta_{2}-n_{2} \quad 0 \leq \eta_{2} \leq 1
$$

The value for $n_{2}$ is obtained from the microphone - signal generator cross-correlation curve in figure 36 . The time delay, $T_{2}$, to the first maximum is used to solve for $\eta_{2}$. Notice that the location of the first maximum was defined as the midpoint between two cross-over points in the correlation function. By using two cross-over points, irregularities in the correlation function trace were averaged.

Figure 36 represents the same operating condition as in figure 34 . The background noise which dominated in the first figure has been eliminated from the second figure. To verify that the background noise did not influence the results, the cross-correlation function for microphone $\mathrm{m}_{2}$ was measured at each test condition with the acoustic source turned off.

Cross-correlating $P_{1}$ and $V_{S}$, it can be shown that

$$
\Delta^{\prime}=\left(\eta_{1}+n_{2}\right)-\Lambda-\Delta_{1}, 0 \leq \eta_{1} \leq \infty
$$

Here the number of integer cycles phase delay is referenced to $m_{2}$. Hence, $\eta_{2}-n_{1}$ can exceed one cycle.

Finally,

$$
\Delta_{T}^{\prime}-\Delta_{T}=\left[\Delta^{\prime}+\left(\Delta_{1}-\Delta_{2}\right)\right]-\Delta_{T}
$$

Substituting equation (D.10) and (D.9) for $\Delta^{\prime}$ and $n_{2}$ gives

$$
\Delta_{T}^{\prime}-\Delta_{T}=\left(\eta_{1}-\eta_{2}\right)-\Delta_{T}
$$


Consider the net pressure, $P$, sensed by microphone $\mathrm{m}_{2}$ in figure 8 to contain a direct acoustic ray represented by amplitude $B_{2}$, a reflected wave represented by $B_{2, R}$, and the background noise given by $\mathrm{P}_{2}^{\prime}$. If the microphone output voltage is cross-correlated with the acoustic driver voltage then the cross-correlation function between $\mathrm{m}_{2}$ and the driver signal is similar to equation D.7. In the present case,

$$
\begin{aligned}
S_{2, s}(\tau)=G_{2} V_{0}\{ & <B_{2} \cos \left[\omega t-2 \pi\left(\Lambda+\Delta_{2}\right)\right] \cos \left[\omega\left(t+\tau_{R}\right)\right]>+ \\
& \left.<B_{2, R} \cos \left[\omega t-2 \pi\left(\Lambda+\Delta_{2}+\Delta_{R}\right)\right] \cos \left[\omega\left(t+\tau_{R}\right)\right]>\right\}
\end{aligned}
$$

As in Appendix D, the microphone sensitivity, $G_{2}$, has been used to link the microphone acoustic pressure input signal and voltage output signal.

The second term in equation E.l corresponds to the cross-correlation between the reflected acoustic signal arriving at the microphone and the acoustic driver voltage signal. Note that the reflected signal has an arbitrary phase shift $\Delta_{R}$, relative to the direct acoustic ray. The cross-correlation between the periodic driver signal and the random background noise is zero so this term is not included in equation E.l. Replacing the trigonometric terms in equation E.1 by the complex notation for the cosine function and noting that the time average of the periodic terms is zero gives

$$
\begin{aligned}
S_{2, S}(\tau)=\frac{1}{2} G_{2} V_{0}\{ & B_{2} \cos \left[\omega \tau_{R}+2 \pi\left(\Lambda+\Delta_{2}\right)\right]+ \\
& B_{2, R} \cos \left[\omega \tau_{R}+2 \pi\left(\Lambda+\Delta_{2}+\Delta_{R}\right)\right]
\end{aligned}
$$

The importance of the second term in equation E.2 can be determined by considering the reflected wave to be $n \pi / 2$ radians out of phase with the direct propagating wave arriving at microphone $\mathrm{m}_{2}$. Under this condition, the reflected wave has a maximum effect on the zero cross-over points used to determine the phase difference, $n_{2}$. Hence, if $2 \pi \Delta_{R}=n \pi / 2$, where $n=1$, 3,5 , etc., then the zero cross-over condition, $s_{2, S}\left(\tau_{R}\right)=0$ gives 


$$
\begin{aligned}
S_{2, S}(\tau)=0= & \cos \left[\omega \tau_{R}+2 \pi\left(\Lambda+\Delta_{2}\right)\right]+ \\
& \frac{B_{2, R}}{B_{2}} \cos \left[\omega \tau_{R}+2 \pi\left(\Lambda+\Delta_{2}\right)+\frac{n \pi}{2}\right]
\end{aligned}
$$

Simplifying the above equation gives

$$
\frac{B_{2}}{B_{2, R}}=\tan \left[\omega \tau_{R}+2 \pi\left(\Lambda+\Delta_{2}\right)\right]
$$

Solving for the phase difference, ${ }^{\tau} \mathrm{R}$, between acoustic driver signal and the combined direct and reflected sound waves gives

$$
\tau_{R} f=\frac{1}{2 \pi} \arctan \left(B_{2} / B_{2, R}\right)-\left(\Lambda+\Delta_{2}\right)
$$

Here, the time difference, $\tau_{R}$, has been multiplied by the frequency, $f$, to express the zero cross-over delay point in the cross-correlation function in terms of cycles.

In the absence of a reflected wave the zero cross-over point is determined by the equation

$$
S_{2, s}(\tau)=0=\cos \left[\omega t-2 \pi\left(\Lambda+\Delta_{2}\right)\right]
$$

Solving for the phase difference between the acoustic driver and the direct sound wave gives

$$
\tau_{D} f=\frac{1}{4}-\left(\Lambda+\Delta_{2}\right)
$$

The difference between $\tau_{D}$ and $\tau_{R}$ represents the error in identifying the zero cross-over point in the presence of the reflected wave. The error, in cycles, is

$$
\left(\tau_{D}-\tau_{R}\right) f=\frac{1}{4}-\frac{1}{2 \pi} \arctan \left(B_{2} / B_{2, R}\right)
$$

It now remains to determine the ratio of direct-to-reflected sound wave amplitude which represents the experimentally observed input to the right side of equation E.8. Free-field decay measurements indicated a variation of 
$\pm 0.5 \mathrm{~dB}$ about the mean line defining the ideal inverse square-law curve. A worst case calculation would assume these deviations to be generated by direct and reflected waves which are either in phase or $180^{\circ}$ out of phase. In the first case the sound pressure level is a maximum corresponding to the $+.5 \mathrm{~dB}$ variation. Here the noise level is controlled by the amplitude coefficient $\left(B_{2}+B_{2, R}\right)^{2}$. For the $180^{\circ}$ phase shift, corresponding to the $-0.5 \mathrm{~dB}$ deviation, the noise is controlled by the amplitude coefficient $\left(B_{2}-B_{2, R}\right)^{2}$. The $1 \mathrm{~dB}$ difference between the +0.5 and -0.5 deviations defines the ratio of the amplitude coefficients given by the expression

$$
1 d B=10 \log \left[\frac{\left(B_{2}+B_{2, R}\right)^{2}}{\left(B_{2}-B_{2, R}\right)^{2}}\right]
$$

Solving for the ratio of the reflected-to-direct sound wave amplitude gives $B_{2, R} / B_{2}=0.0574$.

Substituting the calculated wave amplitude ratio into equation E. 8 gives $\left(\tau_{D}-\tau_{R}\right) f=0.0091$ as the phase error in the zero cross-over point used to determine $\eta_{2}$ in equation 10 . This error is comparable to the experimental accuracy of the phase measurement and is less than the 0.02 cycle accuracy required in the experiment. Based on the error analysis in Appendix $F$, this would result in at most a $0.8^{\circ}$ error in the experimentally determined value of $\theta_{\mathrm{c}}$. 
Error Analysis for Shear Layer Angle Correction Experiment

It is worthwhile to perform an error analysis to determine the sensitivity of the corrected angle, $\theta_{c}$, to errors in the measured phase difference in equation 10 . Based on the chain rule the variation of $\theta_{c}$ relative to the phase difforence is controlled by the equation

$$
\frac{d \theta_{c}}{d \Delta^{*}}=\frac{d \theta_{c}}{d x_{0}} \frac{d x_{0}}{d \mu_{1}} \frac{d \mu_{1}}{d \Delta^{*}}
$$

where

$$
\Delta^{*}=\eta_{1}-\eta_{2}-\Delta_{T}
$$

Using the corresponding expressions for $\theta_{c}$ (equation 7), $x_{o}$ (equation 8) and $\mu_{1}$ (equation 6 ), the above expression becomes

$$
\frac{d \theta_{c}}{d \Delta^{*}}=-\left[\frac{h}{x_{0}^{2}+h^{2}}\right]\left[\frac{r \sin \theta_{m}-h}{\sin ^{2}\left(\mu_{1}+\theta_{m}\right)}\right]\left[\frac{c_{0}}{l f \cos \mu_{1}}\right]
$$

Simplication gives

$$
\frac{d \theta_{c}}{d \Delta^{*}}=-\sin \theta_{c} \frac{(r / h)\left(\sin \theta_{m}-1\right)}{\sin ^{2}\left(\mu_{1}+\theta_{m}\right)} \frac{c_{0}}{\ell f \cos \mu_{1}}
$$

The error in $\theta_{c}$ will be calculated for the case of $\theta_{\mathrm{m}}=90^{\circ}$ where the magnitude of the phase difference is a minimum (see figure 37). Small errors in the measured phase difference are then significant relative to the magnitude of the phase difference. Letting $\theta_{\mathrm{m}}=90^{\circ}$, $\ell=0.48 \mathrm{~m}, \mathrm{M}=0.4$ and $h / r=0.25$ (based on Test Configuration 1 ), the sensitivity of $\theta_{c}$ to changes in the phase difference is estimated by the differential expression $\mathrm{d} \theta_{\mathrm{C}}=$ $-1.6 \mathrm{~d}\left(\Delta^{*}\right)$. Here, $\mathrm{d} \theta_{c}$ is expressed in radians.

Assuming a 0.02 cycle error in the measurement of $\Delta^{*}$ the error in $\theta_{c}$ is calculated to be 0.032 radians or $1.8^{\circ}$. This is small in comparison to the magnitude of $\theta_{c}$ which in this case is $72^{\circ}$. Thus, the accuracy of the phase measurements had a negligible effect on the experimentally determined value of $\theta_{c}$. 


\section{REFERENCES}

1. Schlinker, R.H., Amiet, R.K.: Experimental Assessment of Theory for Refraction of Sound by a Shear Layer, NASA CR-145359, 1978. Also AIAA Paper 79-0628.

2. Ribner, H.S.: Reflection Transmission and Amplification of Sound by a Moving Medium. J. Acoust. Soc. Amer., vol. 29, 1957, pp. 435-441.

3. Miles, J.W.: On the Reflection of Sound at an Interface of Relative Motion. J. Acoust. Soc. Amer., vol. 29, 1957, pp. 226-228.

4. Gottlieb, P.: Sound Source Near a Velocity Discontinuity. J. Acoust. Soc. Amer., vol. 32, 1960, pp. 1117-1122.

5. Graham, E.W. and Graham, B.B.: Effect of a Shear Layer on Plane Waves of Sound in a Fluid. J. Acoust. Soc. Amer., vol. 46, 1969, pp. 169-175.

6. Amiet, R.K.: Propagation of Sound Through a Two-Dimensional Shear Layer with Application to Measurements in the Acoustic Research Tunnel. United Technologies Research Center Report UAR-L40, 1972.

7. Amiet, R.K.: Correction of Open Jet Wind Tunnel Measurements for Shear Layer Refraction. Progress in Astronautics and Aeronautics, Ed. Martin Summerfield, 1976, pp. 259-280. Also AIAA Paper 75-532, 1975, and UTRC Report N111208-1, 1974.

8. Jacques, J.R.: Noise from Moving Aircraft: Some Relevant Models. Ph.D. Thesis, Cambridge University, 1975.

9. Tester, B.J. and Morfey, C.L.: Developments in Jet Noise Modeling - Theoretical Predictions and Comparisons with Measured Data. J. Sound and Vib., vol. 46, 1976, pp. 79-103.

10. Amiet, R.K.: Refraction of Sound by Shear Layer. AIAA Paper 77-54. Also, to be published in J. Sound and Vib., vol. 58, No. 3., 1978.

11. Candell, S. M.: Application to Geometrical Techniques to Aeroacoustic Problems. AIAA Paper 76-546, 1976.

12. Tester, B.J. and Burrin, R.H.: On Sound Radiation From Sources in Parallel Sheared Jet Flows. AIAA Paper 75-57, 1975.

13. Mani, R., Clapper, W.S., Stringas, E.J. and Banarian, G.: Development of a Technique for Inflight Jet Noise Simulation - Part II. AIAA Paper 76-532, 1976. 
REFERENCES (Cont'd)

14. Candell, S.M., Guedel, A. and Julienne, A.: Refraction and Scattering in an Open Wind Tunnel Flow. Proceedings of the 6 th International Congress on Instrumentation in Aerospace Simulation Facilities, IEEE, September 1975, pp. 288-299.

15. Ahuja, K.K., Tester, B.J., Tanna, H.K.: The Free Jet as a Simulator of Forward Velocity Effects on Jet Noise. NASA CR-3056, 1978. Also AIAA Paper 77-1266, 1977.

16. Ozkul, A., Yu, J.C.: An Experimental Investigation of Acoustic Radiation from a Source Inside a Large Turbulent Free Jet, J. Acoust. Soc. Amer., vol. 65, part 2, 1979, pp. 336-344.

17. Paterson, R.W., Vogt, P.G., and Folery, W.M.: Design and Development of the United Aircraft Research Laboratories Acoustic Research Tunnel. J. Aircraft, vol. 10, No. 7, 1973, pp. 427-433.

18. Schlinker, R.H., Fink, M.R., and Amiet, R.K.: Vortex Noise from Nonrotating Cylinders and Airfoils. AIAA Paper 76-81, 1976.

19. Paterson, R.W., and Amiet, R.K.: Acoustic Radiation and Surface Pressure Characteristics of an Airfoil Due to Incident Turbulence. AIAA Paper 76-571, 1976.

20. Rajarathnam, N.: Turbulent Jets. American Elsevier Publishing Co., 1976.

21. Cande11, S. M., Guedel, A., and Julienne, A.: Radiation, Refraction and Scattering of Acoustic Waves in a Free Shear Flow. AIAA Paper 76-544, 1976.

22. Paterson, R.W.: Acoustic Research Tunnel Test Summary \#29; Passage of High Frequency Pure Tones Through a Turbulent Round Jet. Report UAR-M112, United Aircraft Research Laboratories, August 1973.

23. Schlinker, R.H.: Transmission of Acoustic Plane-Waves at a Jet Exhaust. J. of Aircraft, vol. 16, No.3, 1979, pp. 188-194.

24. Lighthill, M.J.: On the Energy Scattered from the Interaction of Turbulence with Sound or Shock Waves . Proc. Camb. Phil. Soc., vo1. 49, 1953, pp. 531551 .

25. Howe, M.S.: Mulitple Scattering of Sound by Turbulence and Other Subhomogeneities. J. Sound and Vib., vol. 27, 1973, pp. 455-476.

26. Amiet, R.: Scattering of Sound by Turbulence. United Technologies Research Center Report UTRC79-112, 1979. 
Table I - Refraction Angle Correction Test Conditions.

\begin{tabular}{|c|c|c|c|c|c|}
\hline \multirow{2}{*}{$\begin{array}{c}\text { TEST } \\
\text { GEOMETRY } \\
\text { NUMBER }\end{array}$} & \multicolumn{2}{|c|}{$\begin{array}{l}\text { SOURCE } \\
\text { LOCATION }\end{array}$} & \multirow{2}{*}{$\begin{array}{l}\text { RADIAL } \\
\text { LOCATION } \\
\frac{n}{r}\end{array}$} & \multirow{2}{*}{$\begin{array}{c}\text { MACH } \\
\text { NUMBER } \\
M\end{array}$} & \multirow{2}{*}{$\begin{array}{c}\text { SOURCE } \\
\text { FREOUENCY } \\
k \mathrm{~Hz}\end{array}$} \\
\hline & $\frac{x}{R_{0}}$ & $\frac{h}{R_{0}}$ & & & \\
\hline 1 & 1.33 & 1 & 0.25 & $0.1,0.2,0.3,0.4$ & $1,2.5,5,10$ \\
\hline 2 & 2.66 & 1 & 0.25 & $0.2,0.4$ & \\
\hline 3 & 4.0 & 1 & 0.25 & $0.2,0.4$ & \\
\hline 4 & 1.33 & 0.55 & 0.14 & $0.1,0.2,0.3,0.4$ & \\
\hline 5 & 1.33 & 1.44 & 0.16 & 0.4 & 5. 10 \\
\hline
\end{tabular}

Table II - Refraction Amplitude Correction Test Conditions.

\begin{tabular}{|c|c|c|c|c|c|}
\hline \multirow{2}{*}{$\begin{array}{c}\text { TEST } \\
\text { GEOMETRY } \\
\text { NUMBER }\end{array}$} & \multicolumn{2}{|c|}{$\begin{array}{l}\text { SOURCE } \\
\text { LOCATION }\end{array}$} & \multirow{2}{*}{$\begin{array}{l}\text { SIDELINE } \\
\text { STATION } \\
\frac{n}{y}\end{array}$} & \multirow{2}{*}{$\begin{array}{c}\text { MACH } \\
\text { NUMBER } \\
M\end{array}$} & \multirow{2}{*}{$\begin{array}{c}\text { SOURCE } \\
\text { FREQUENCY } \\
\mathbf{k H z}\end{array}$} \\
\hline & & $\frac{h}{R_{0}}$ & & & \\
\hline 6 & 0.53 & 1 & 0.25 & $0.1,0.2,0.3,0.4$ & $1.25,2.5,5,10$ \\
\hline 7 & 2.66 & 1 & 0.25 & & \\
\hline 8 & 0.53 & 1.67 & 0.42 & $0.1,0.2,0.3$ & t \\
\hline
\end{tabular}


Table III - Turbulence Scattering Test Conditions.

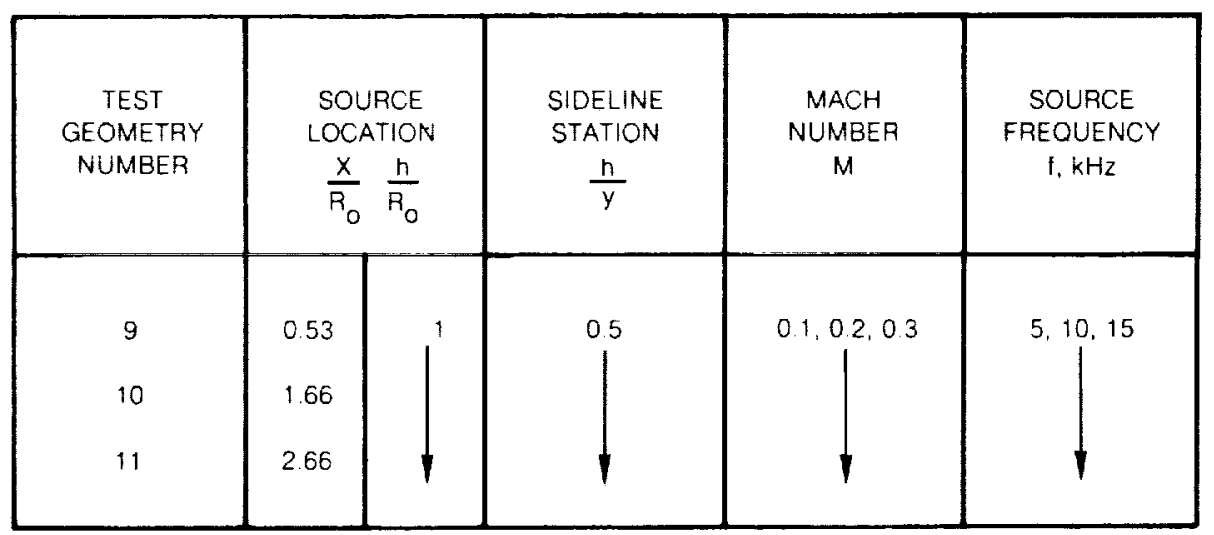



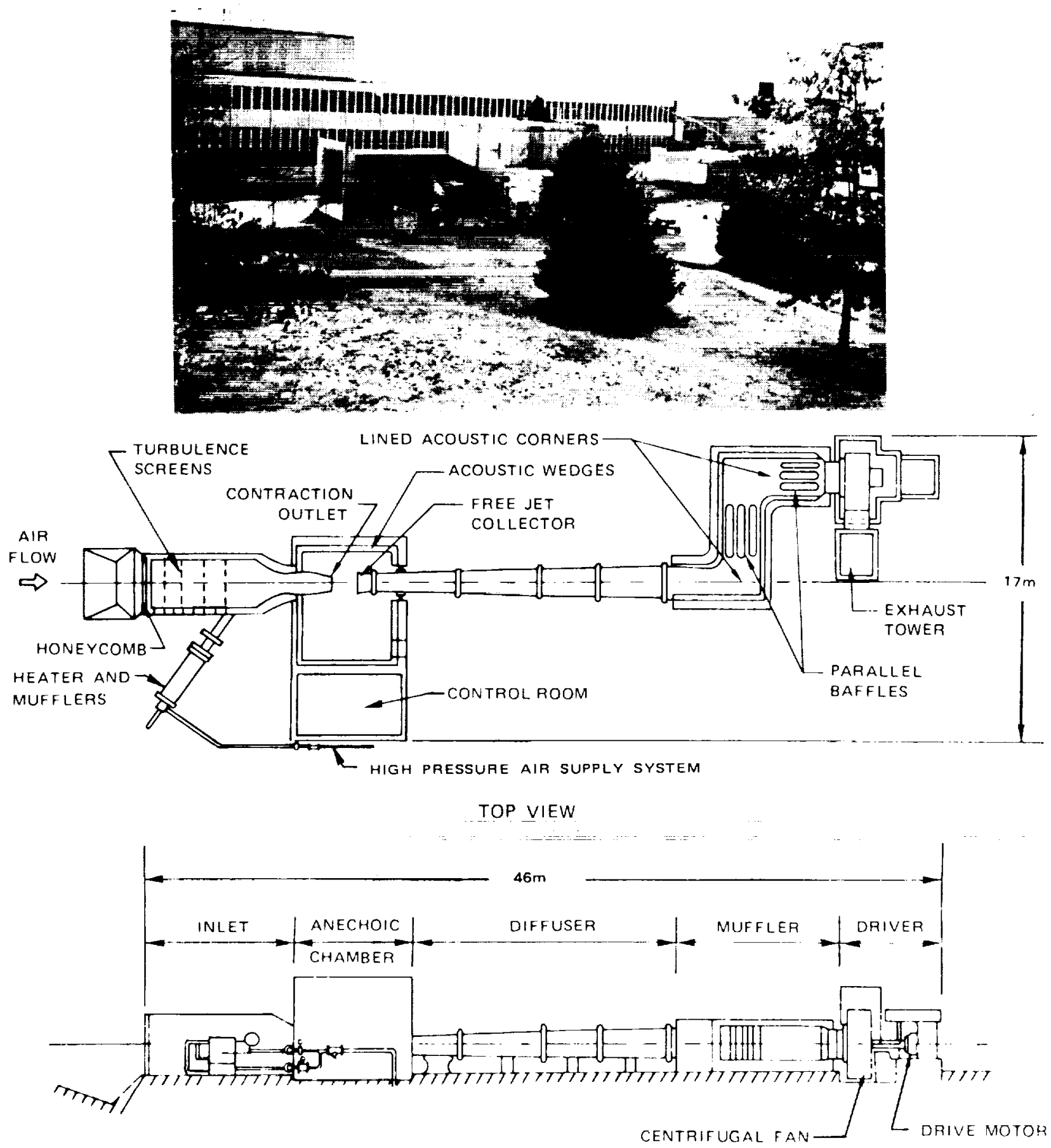

SIDE VIEW

Figure 1 - UTRC Accoustic Research Tunnel. 


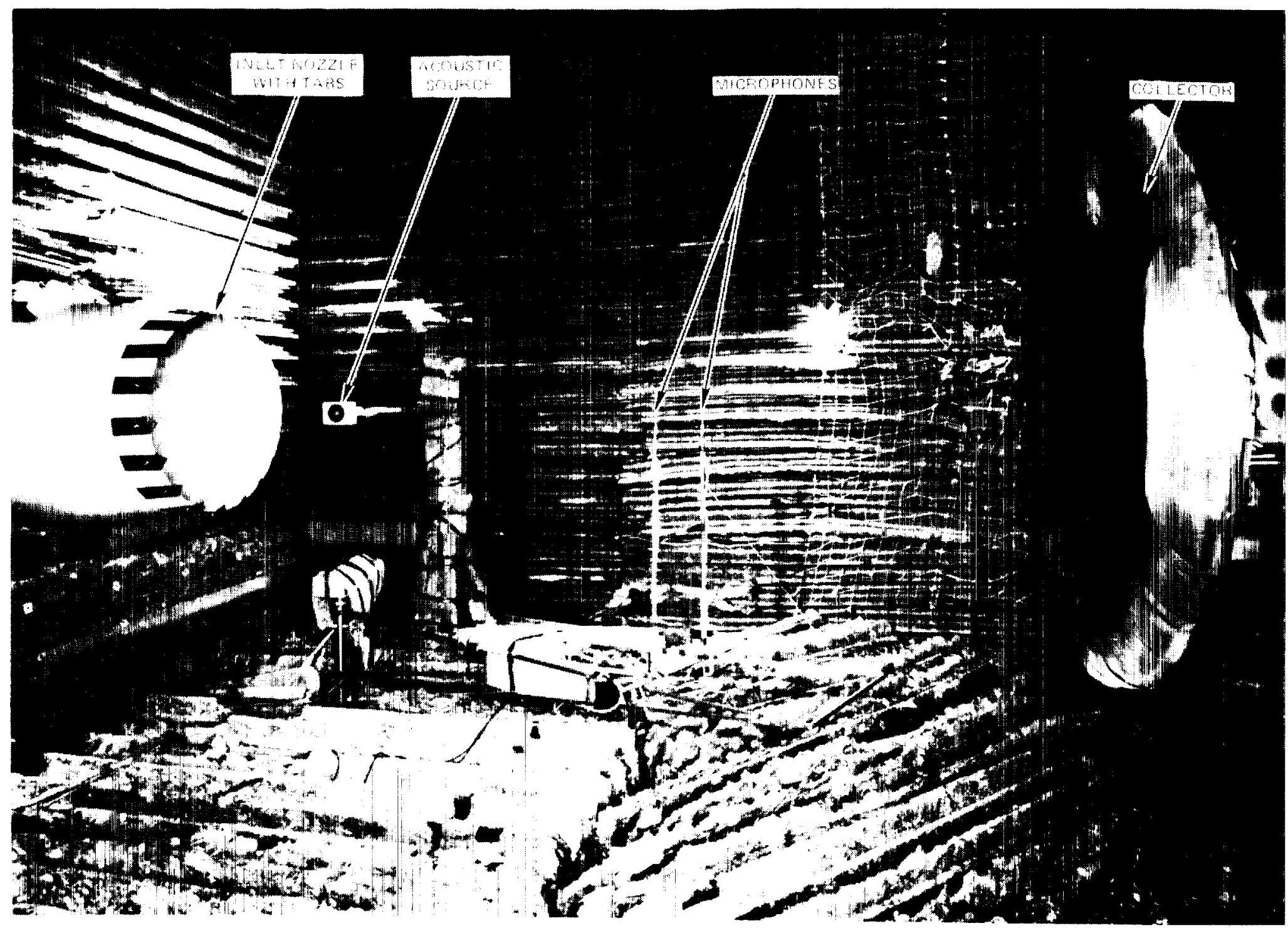

Figure 2 - Anechoic Chamber Test Arrangement for Refraction Angle Correction Experiment. 


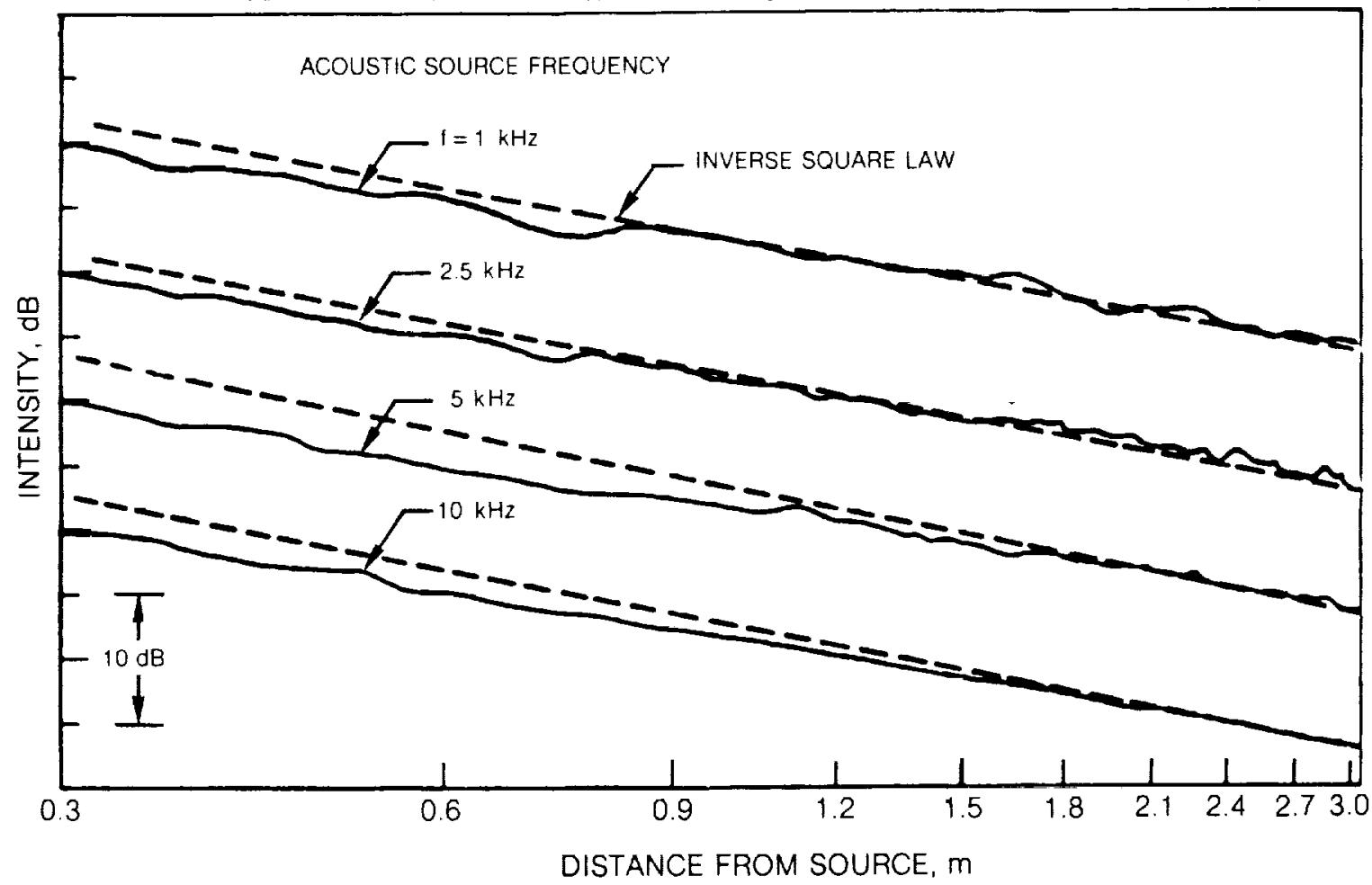

Figure 3- Intensity-Distance Variation for Acoustic Source Employed in Refraction Angle Correction Experiment with Discrete Frequency Excitation. 


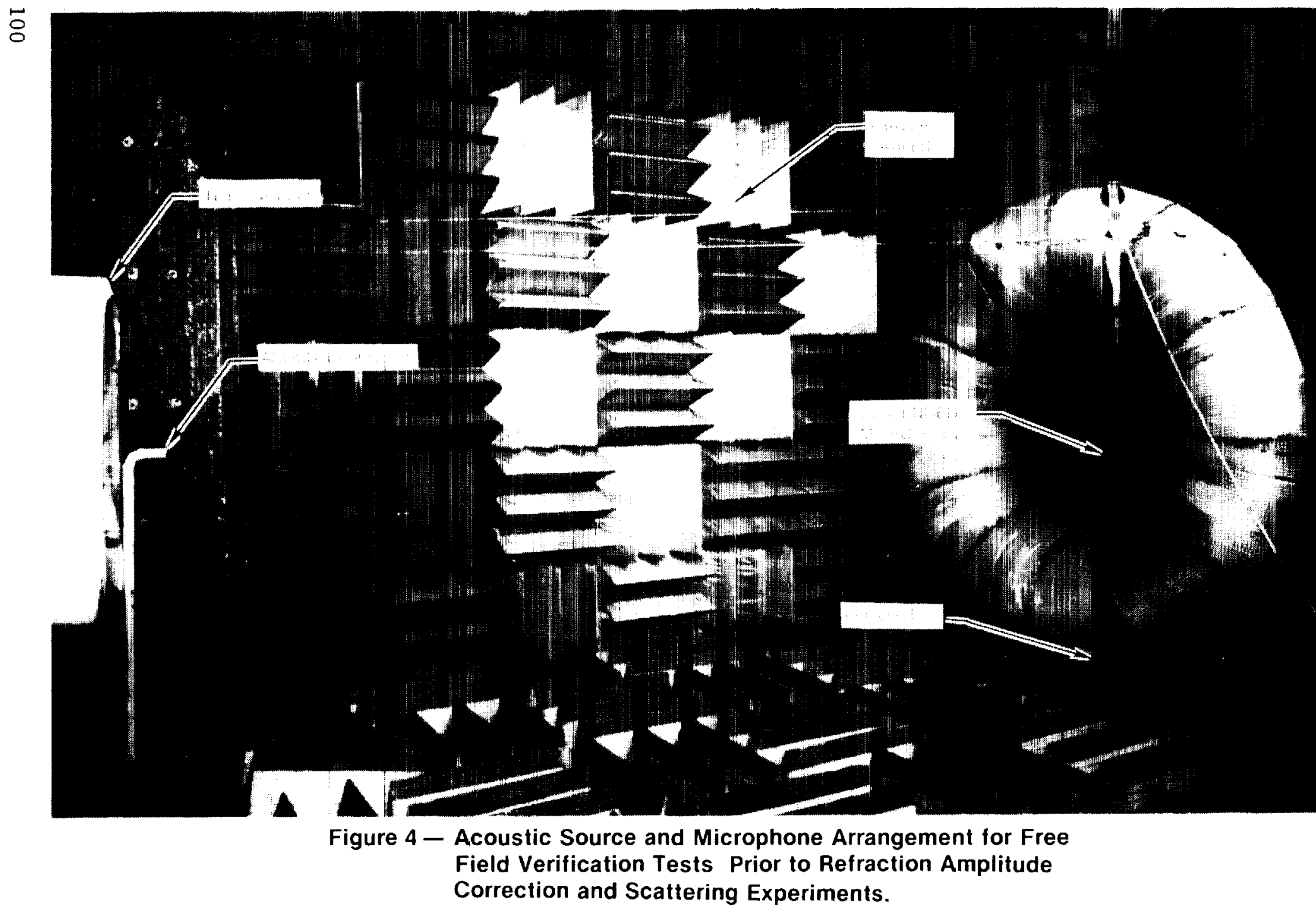




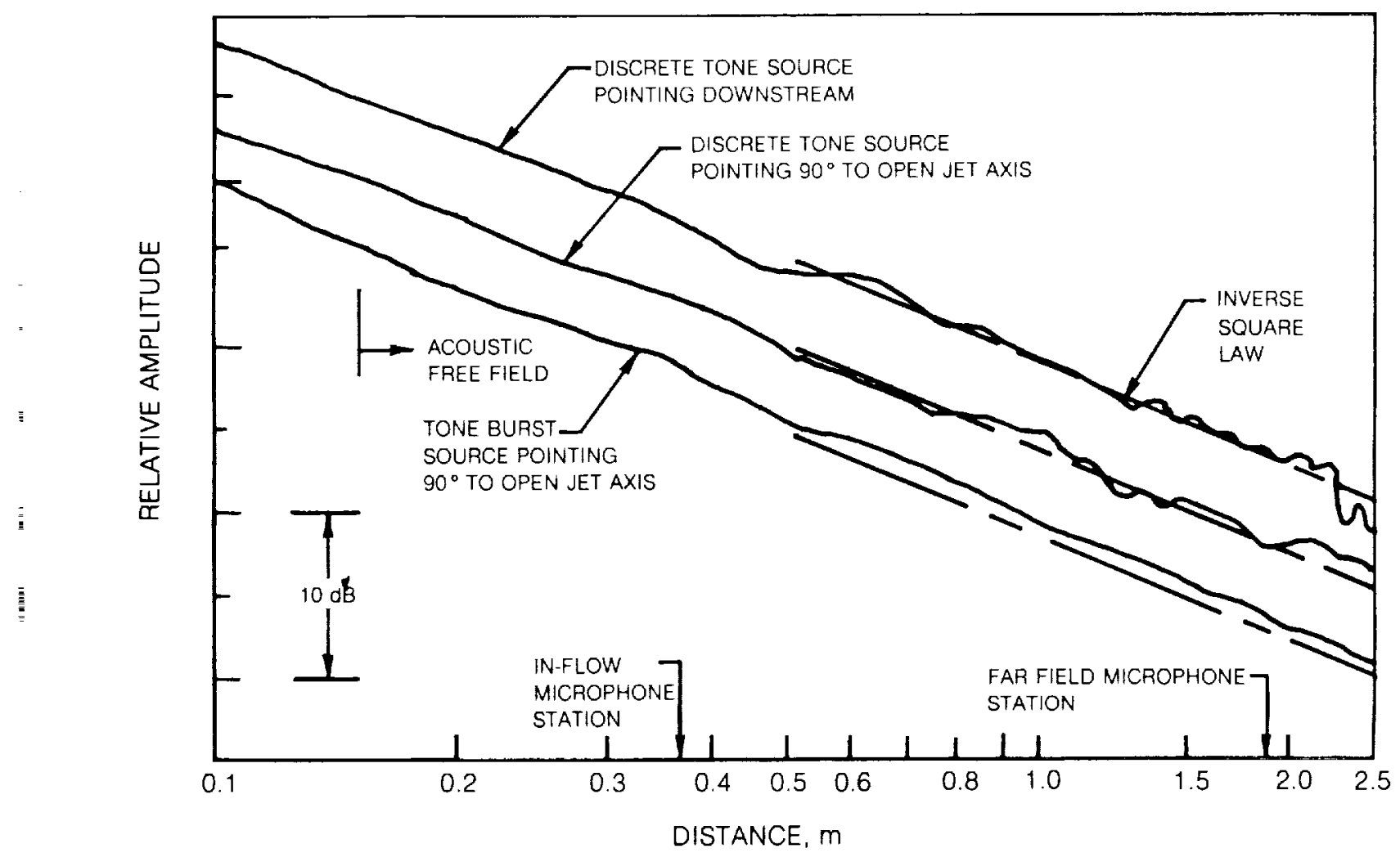

Figure 5 - Intensity Versus Distance Variation for Acoustic Source Employed in Refraction Amplitude Correction Experiment with $1.25 \mathrm{kHz}$ Discrete Tone and Tone Burst, $\theta_{\mathbf{m}}=90^{\circ}$. 

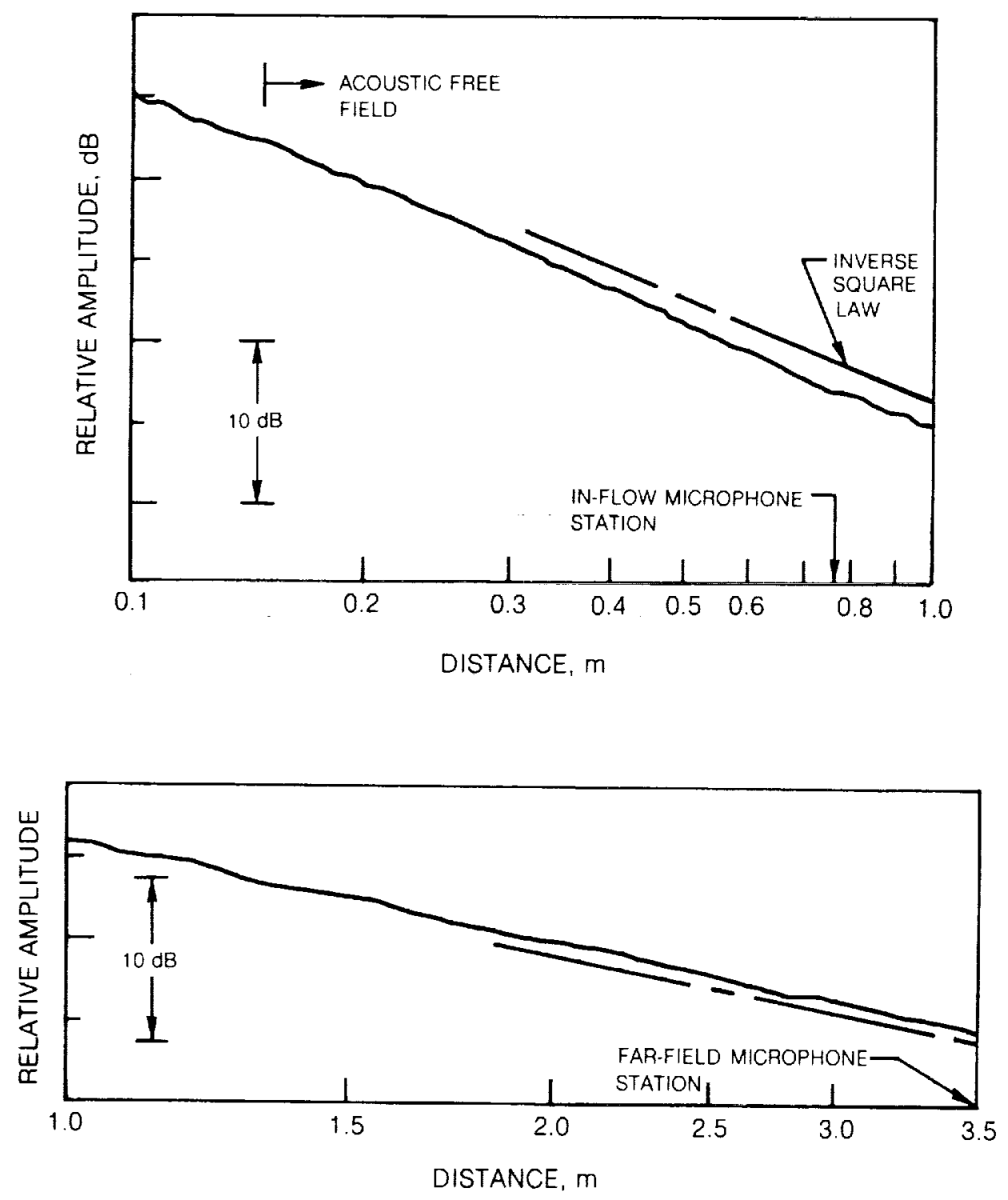

Figure 6 - Intensity Versus Distance Variation for Acoustic Source Employed in Refraction Amplitude

Correction Experiment with $1.25 \mathrm{kHz}$ Tone Burst, $\theta_{\mathrm{m}}=30^{\circ}$. 


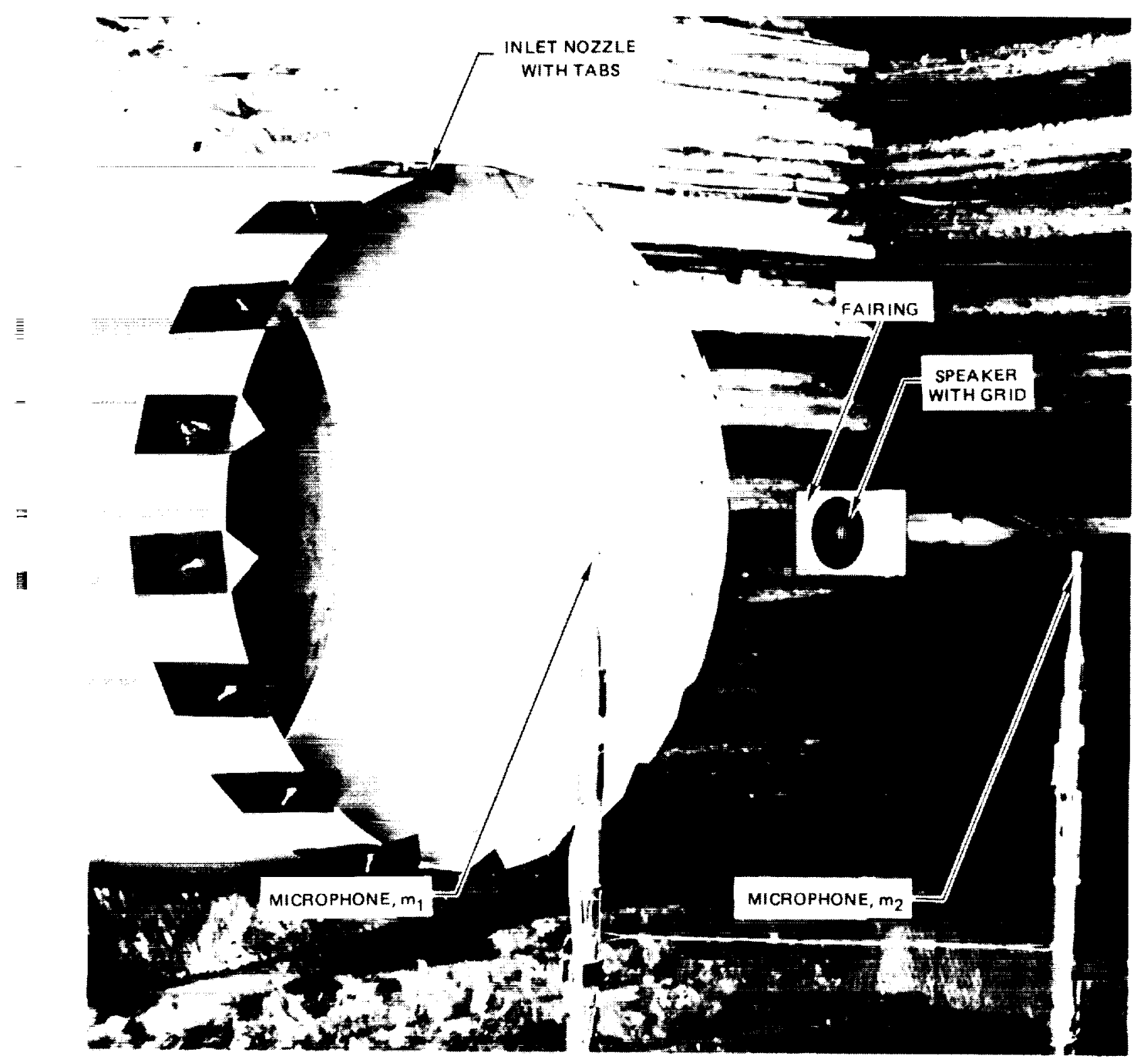

Figure 7 -Acoustic Source and Far Field Microphone Arrangement for Refraction Angle Correction Experiment. 


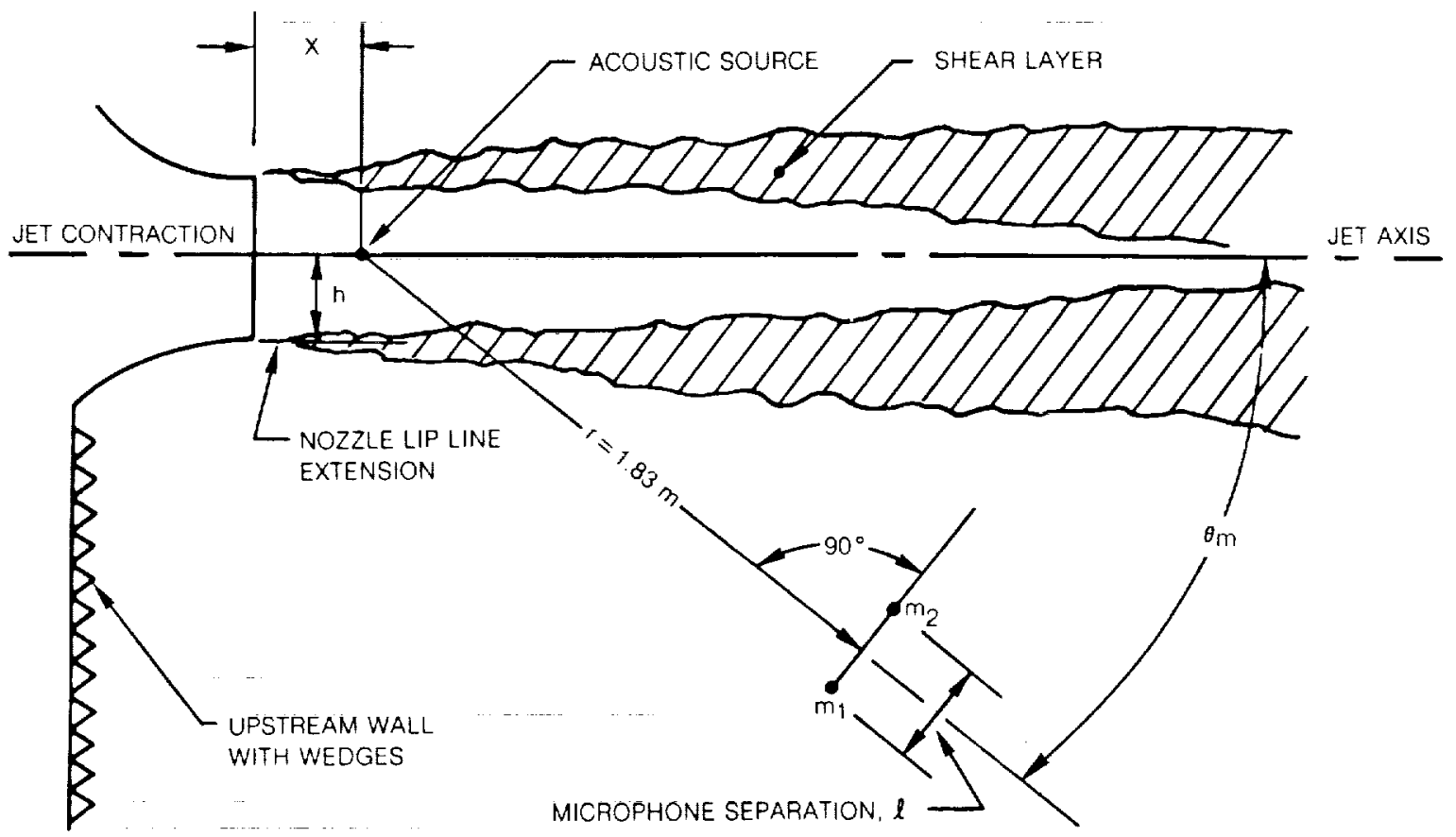

Figure 8 - Plan View of Open Jet, Acoustic Source and Far - Field Microphone Arrangement for Refraction Angle Correction Experiment. 


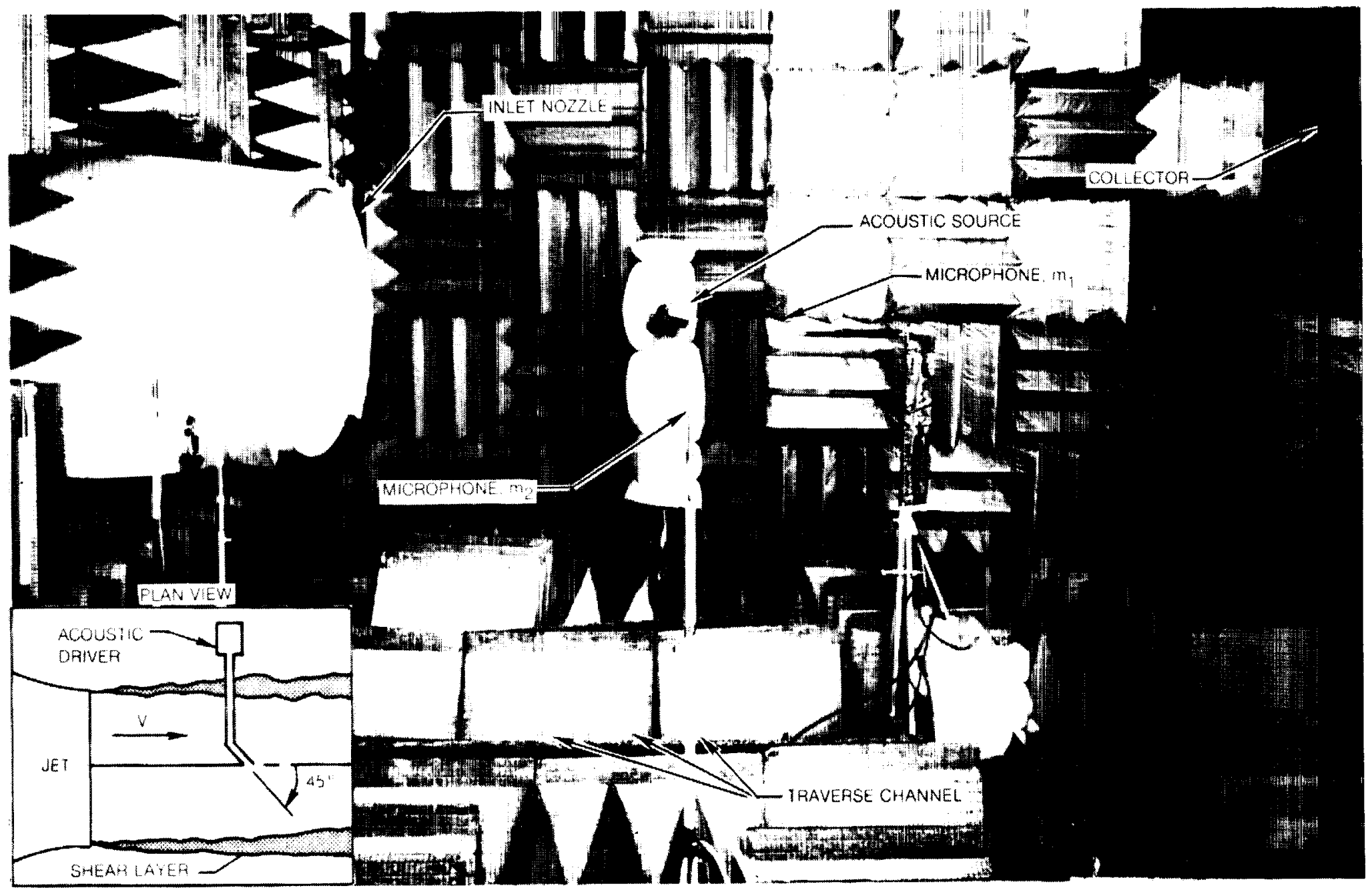

Figure 9 - Anechoic Chamber Test Arrangement for Refraction Amplitude Correction and Scattering Experiment. 


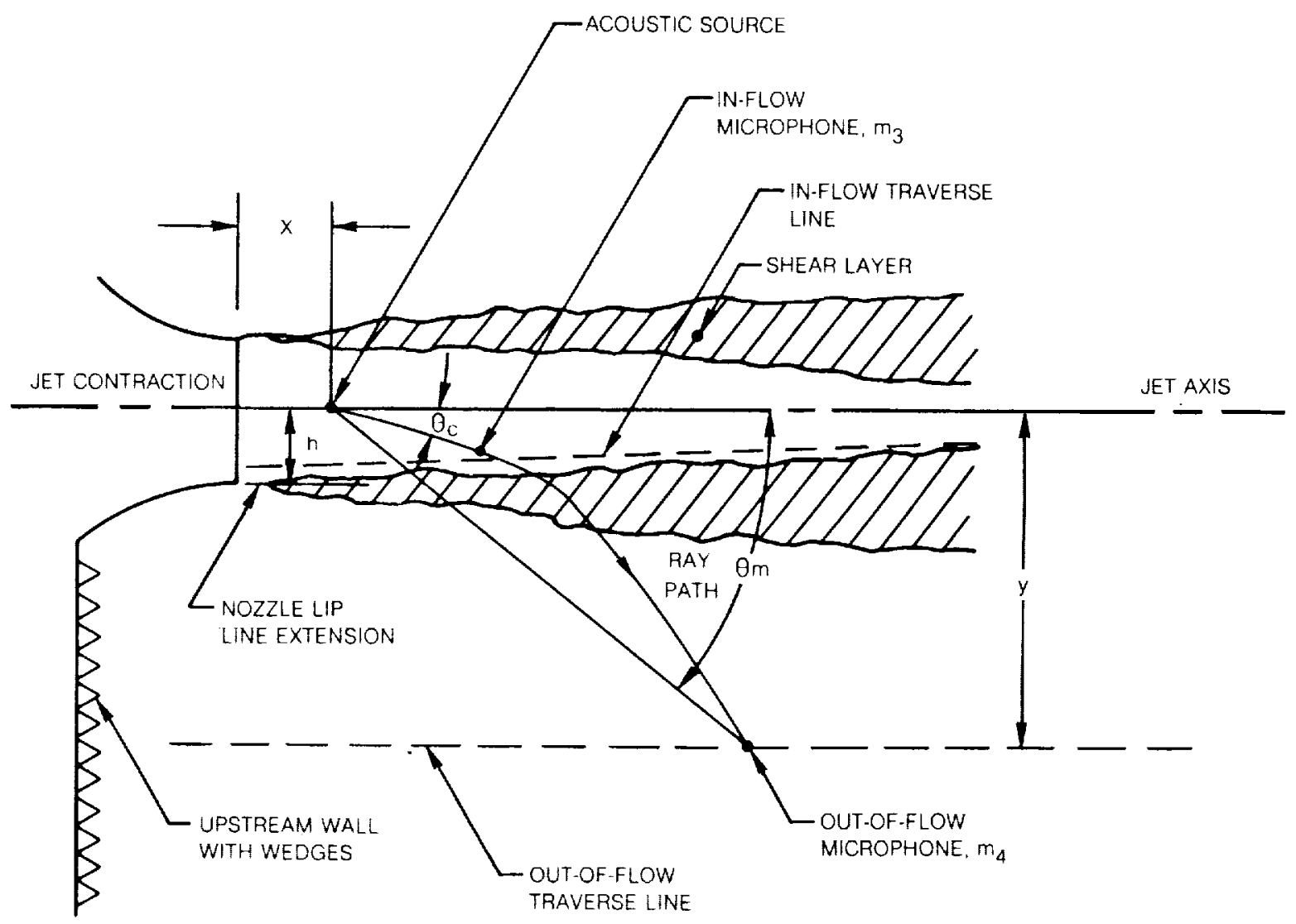

Figure 10 - Plan View of Open Jet, Acoustic Source and Microphone Arrangement for Refraction Amplitude Correction Experiment. 


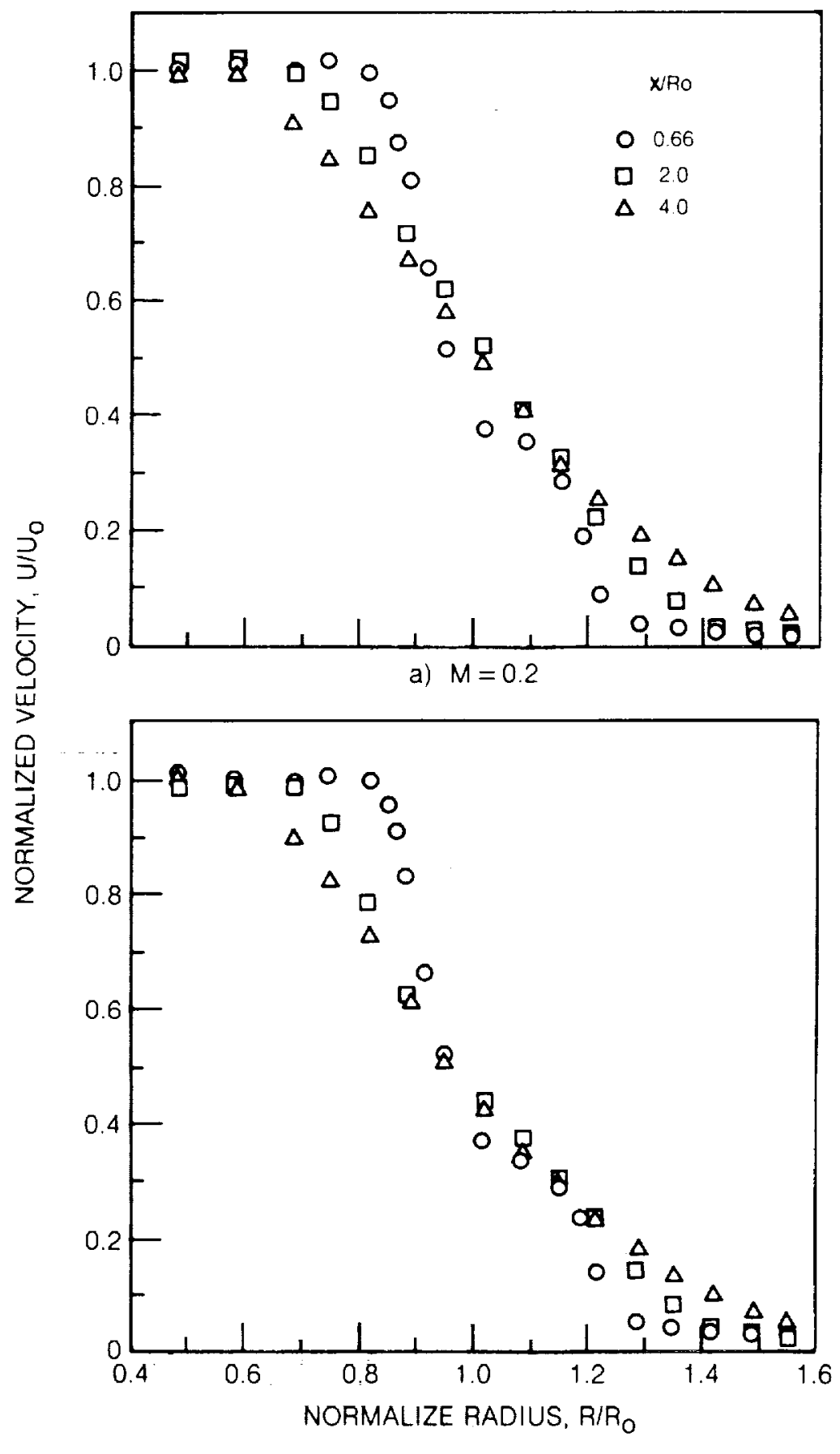

b) $M=0.4$

Figure 11- Variation of Normalized Velocity Profiles with Axial Station, Tabs Installed. 


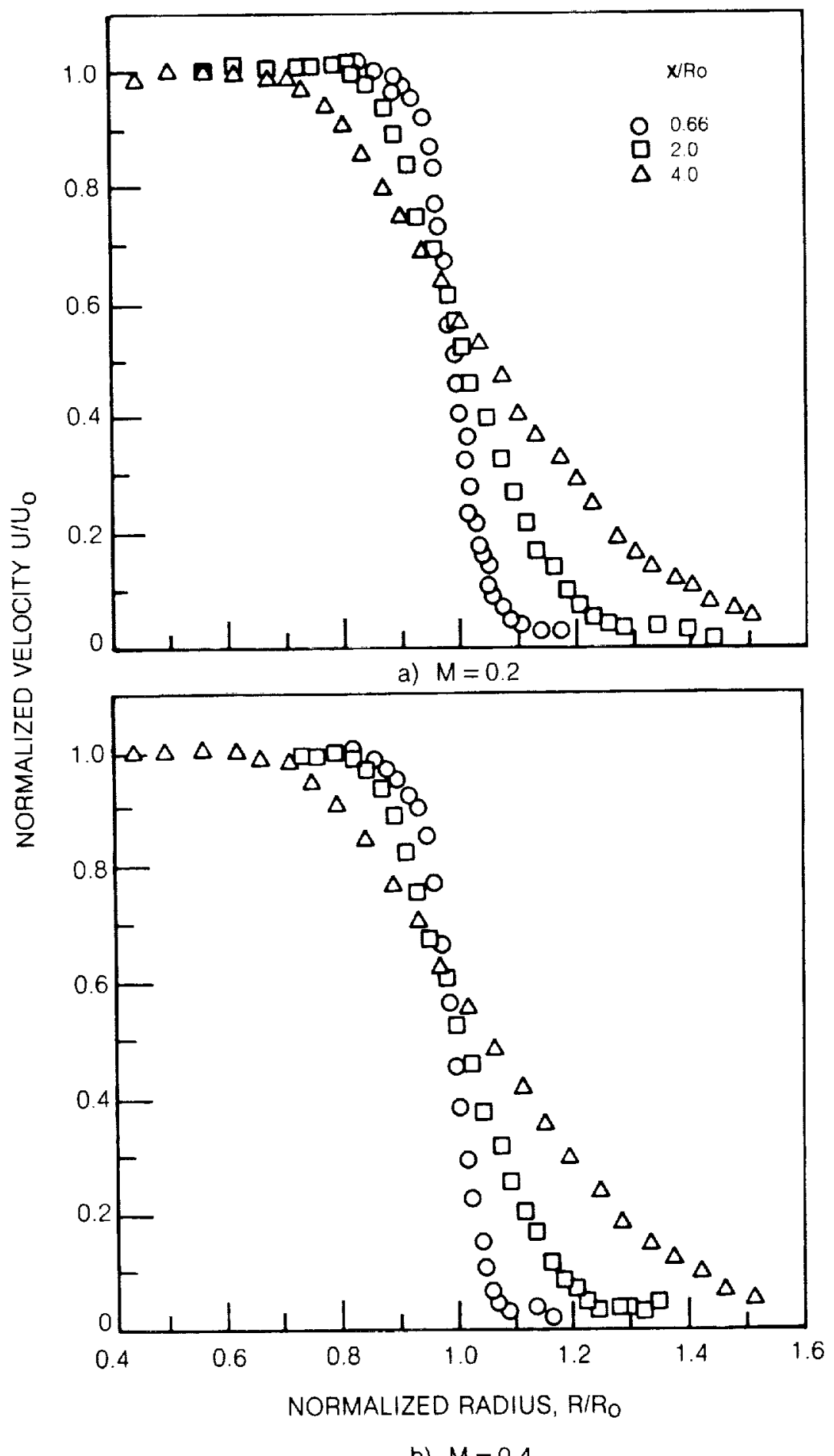

Figure 12 - Variation of Normalized Velocity Profiles with Axial Station, Tabs Removed. 


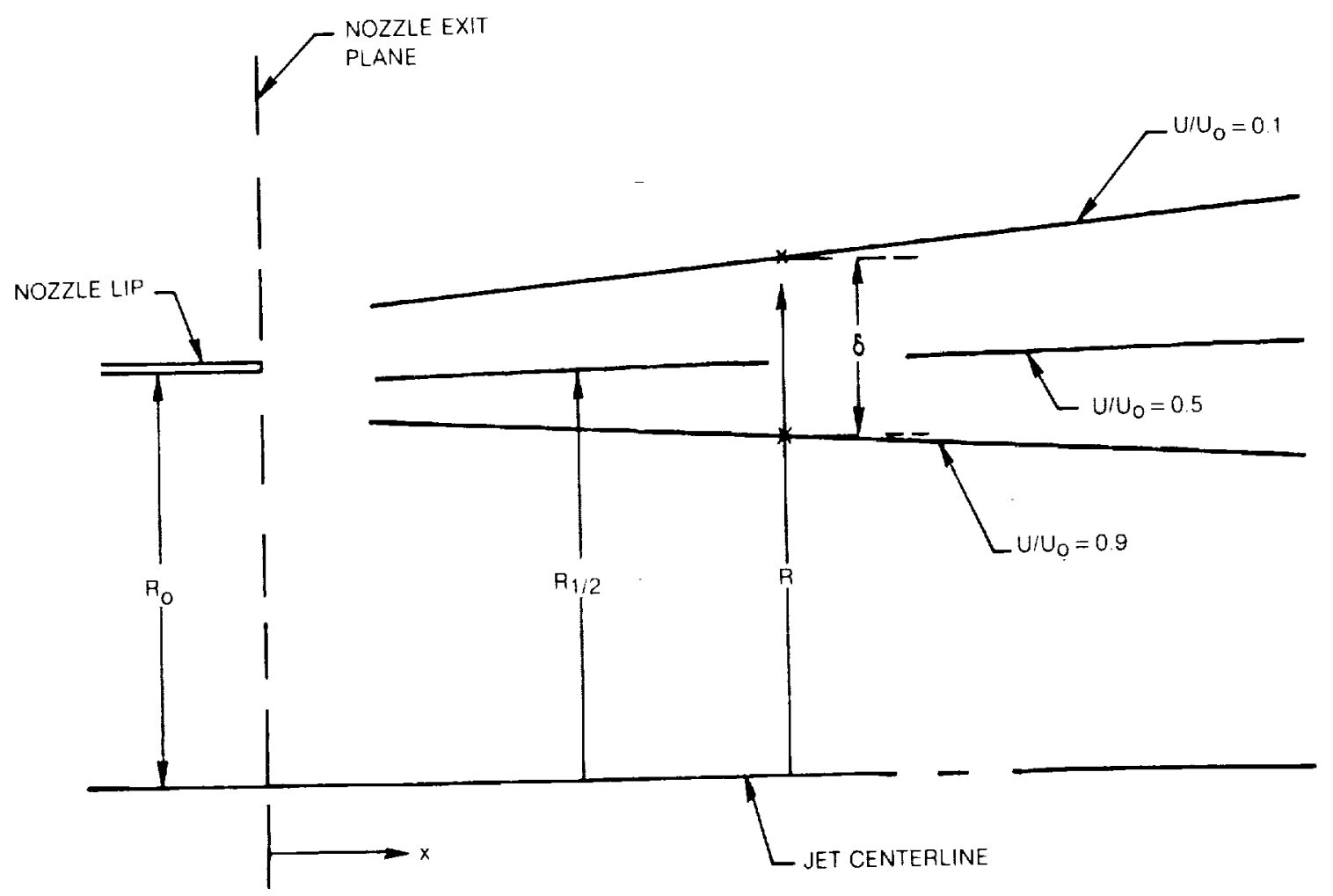

Figure 13 - Shear Layer Conditions. 


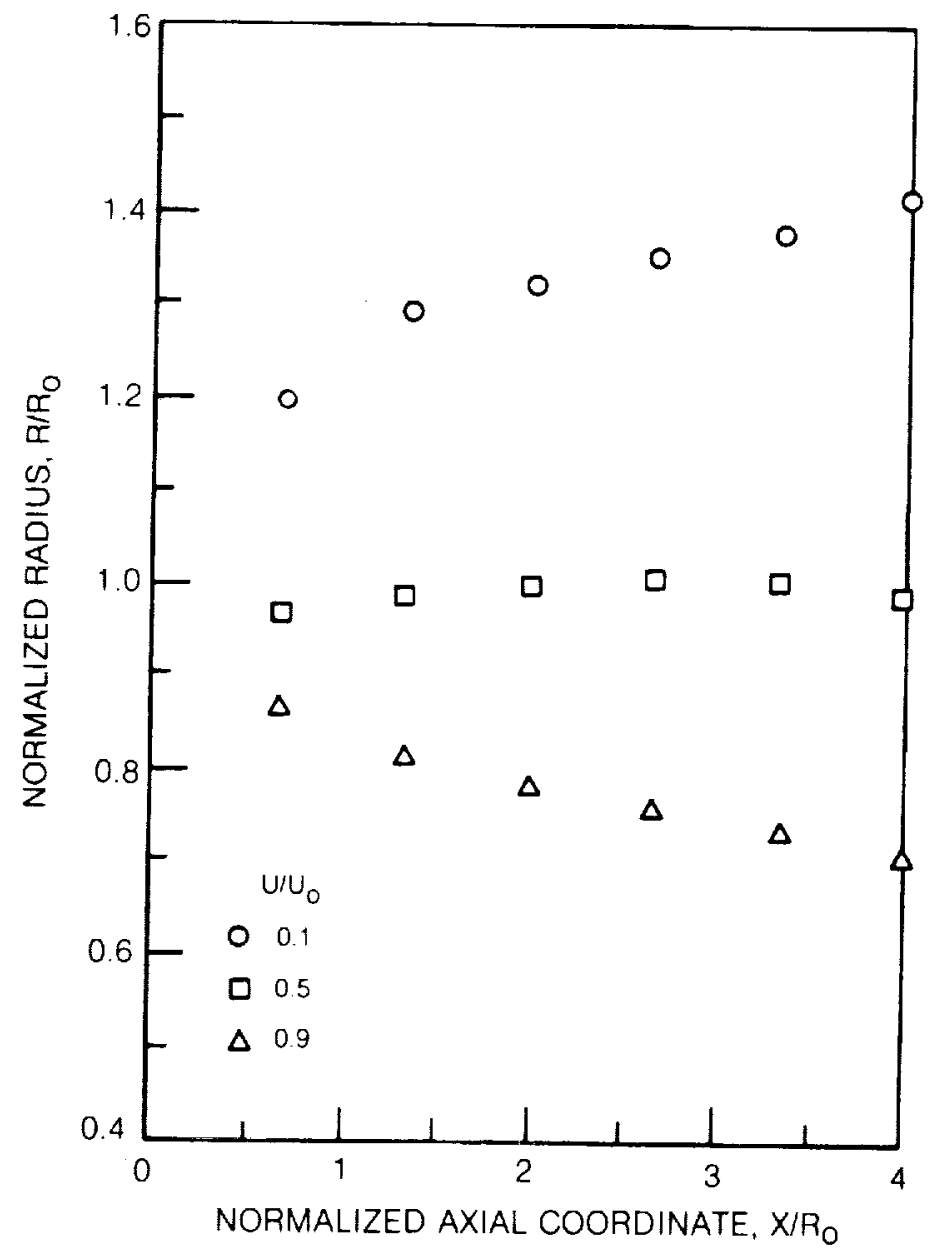

Figure 14 - Axial Variation of Average Shear Layer Characteristics, Tabs Installed. 


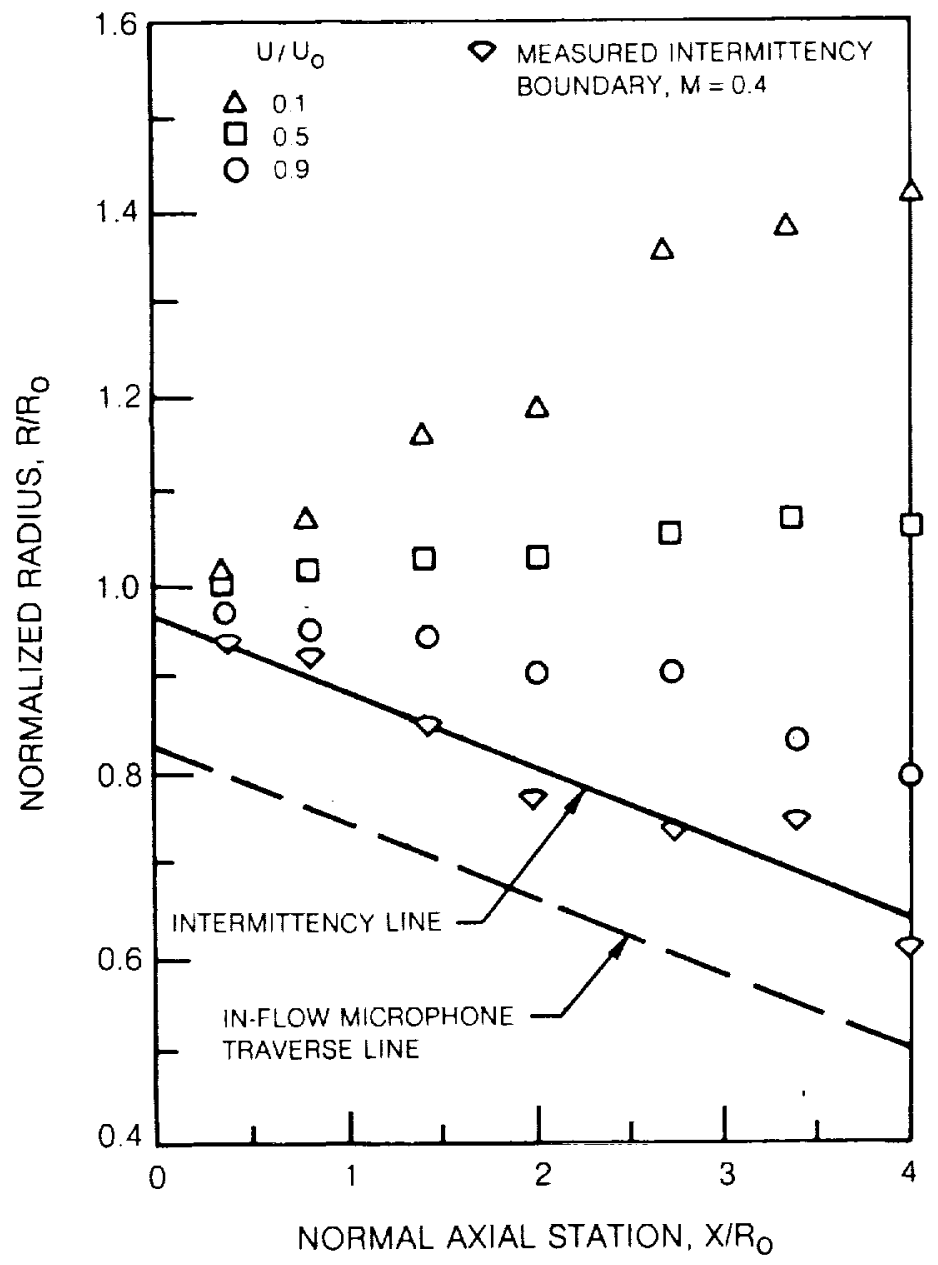

Figure 15 - Axial Variation of Average Shear Layer Characteristics, Tabs Removed. 


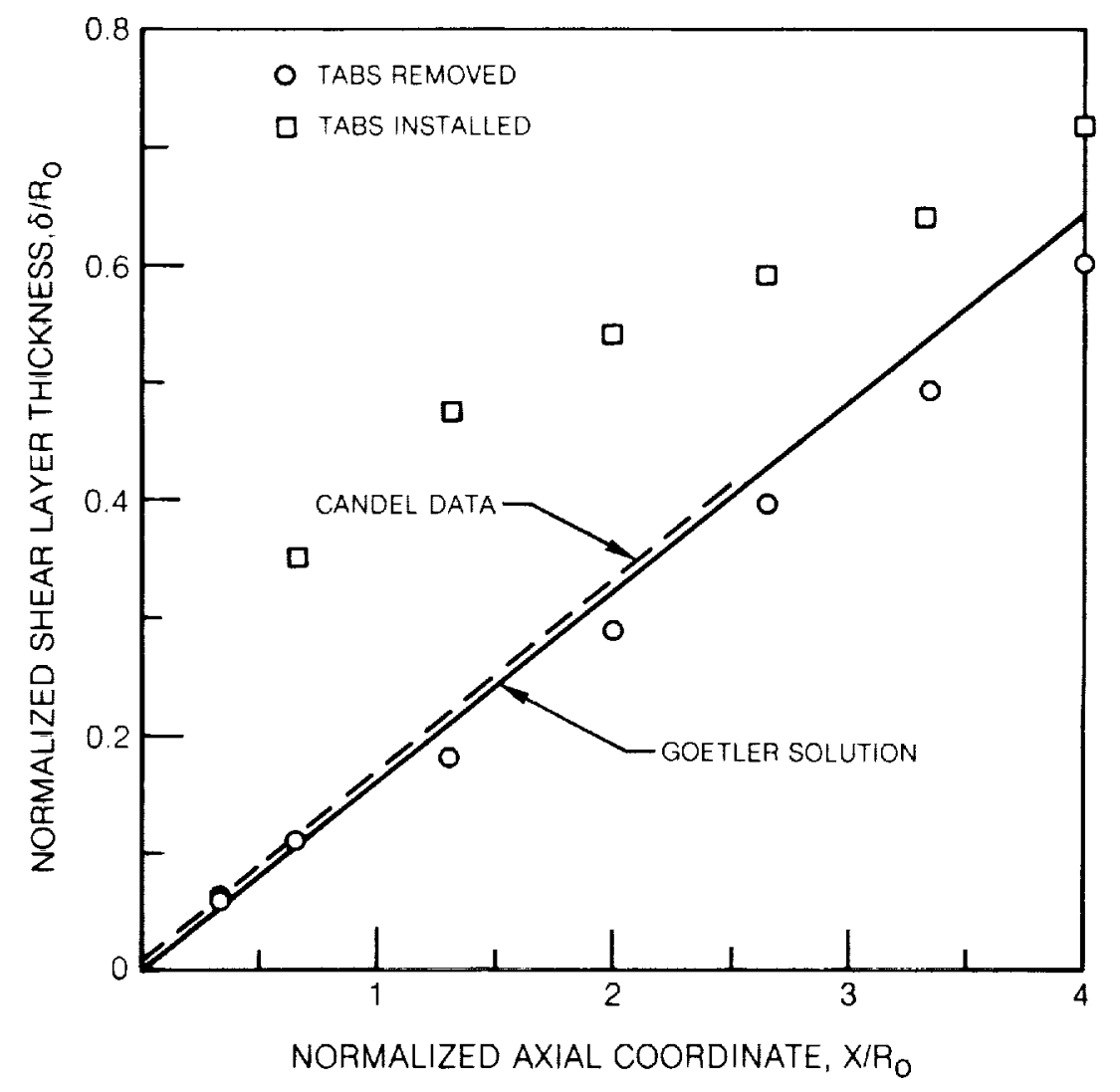

Figure 16 - Axial Variation of Average Shear Layer.Thickness. 


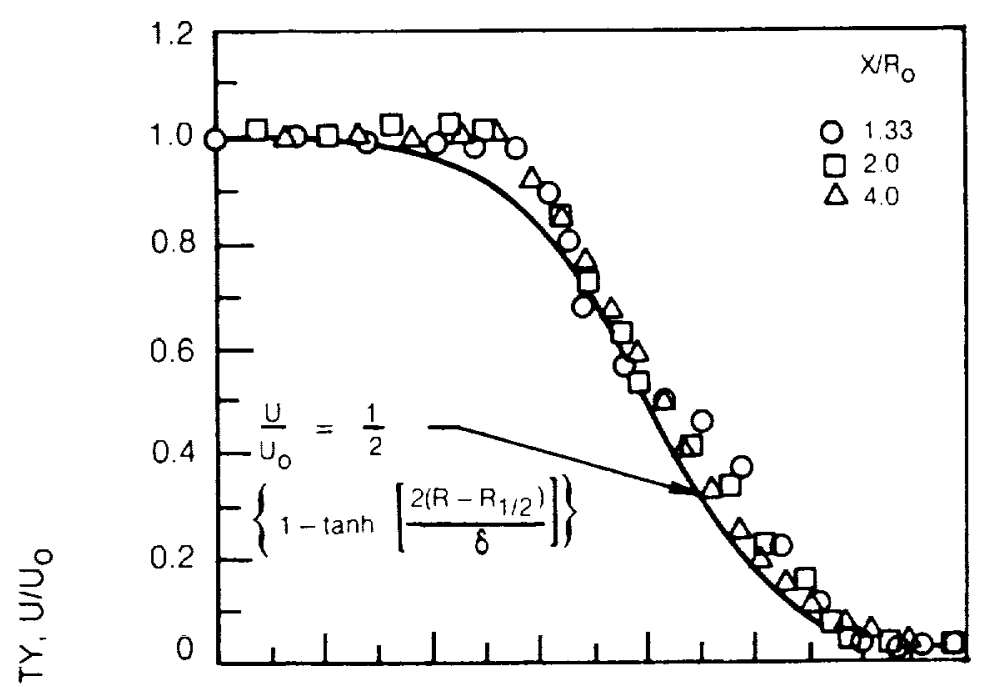

a) $M=0.2$

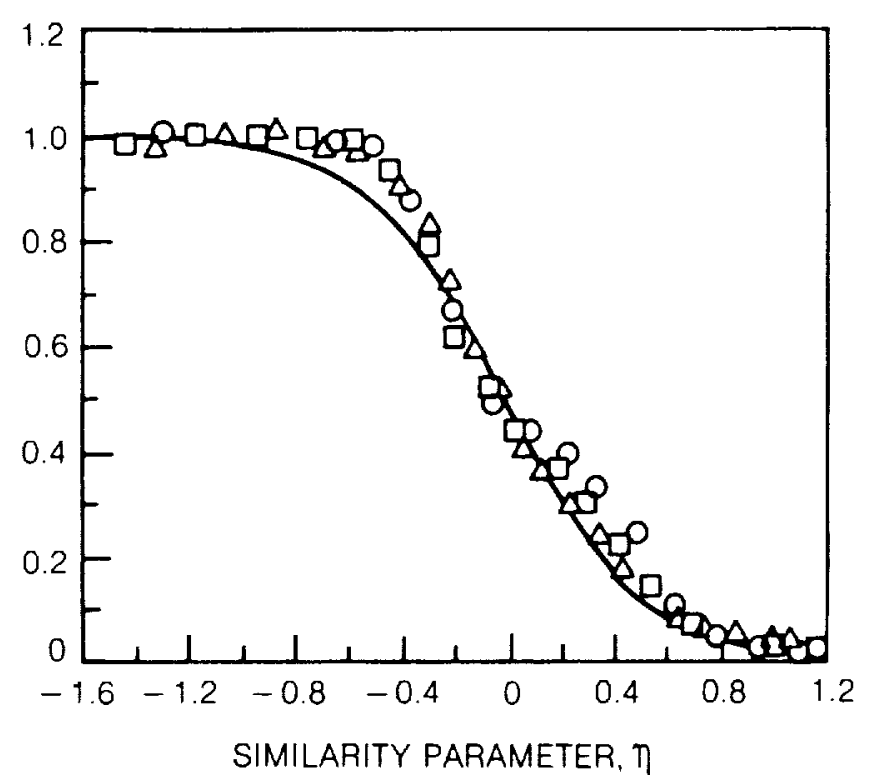

b) $M=0.4$

Figure 17- Similarity of Velocity Profiles at Various Axial Stations. Tabs Installed. 


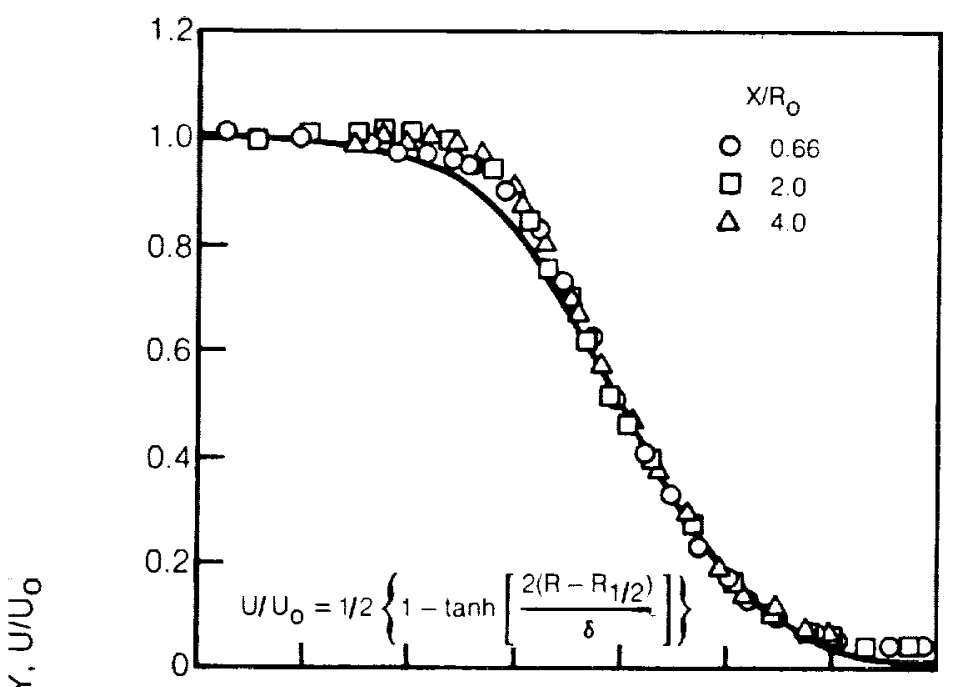

a) $M=0.2$

,

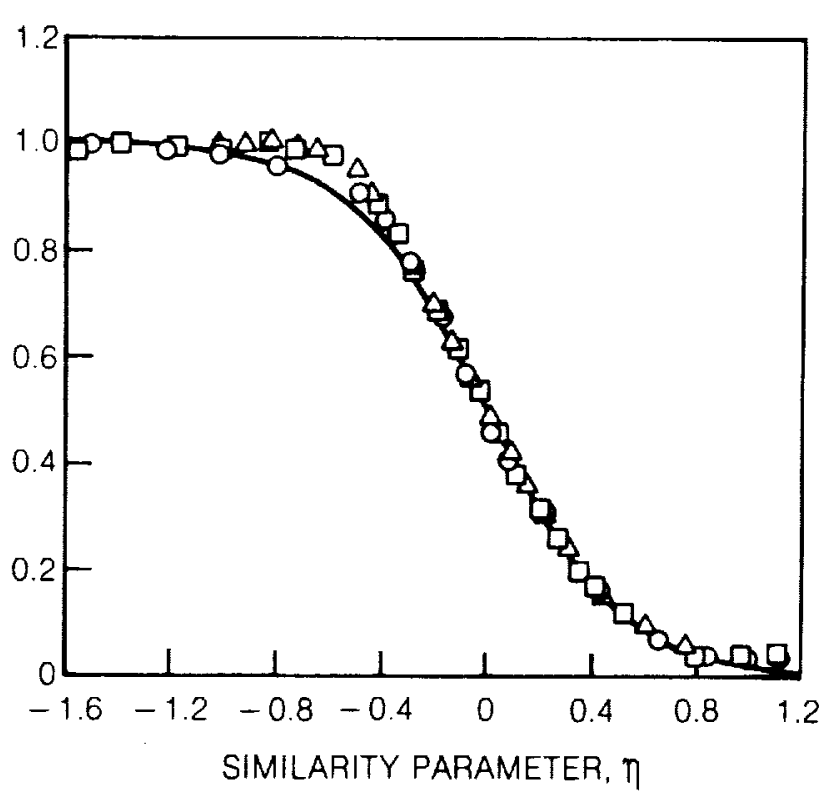

b) $M=0.4$

Figure 18 - Similarity of Velocity Profiles at Various Axial Stations, Tabs Removed. 


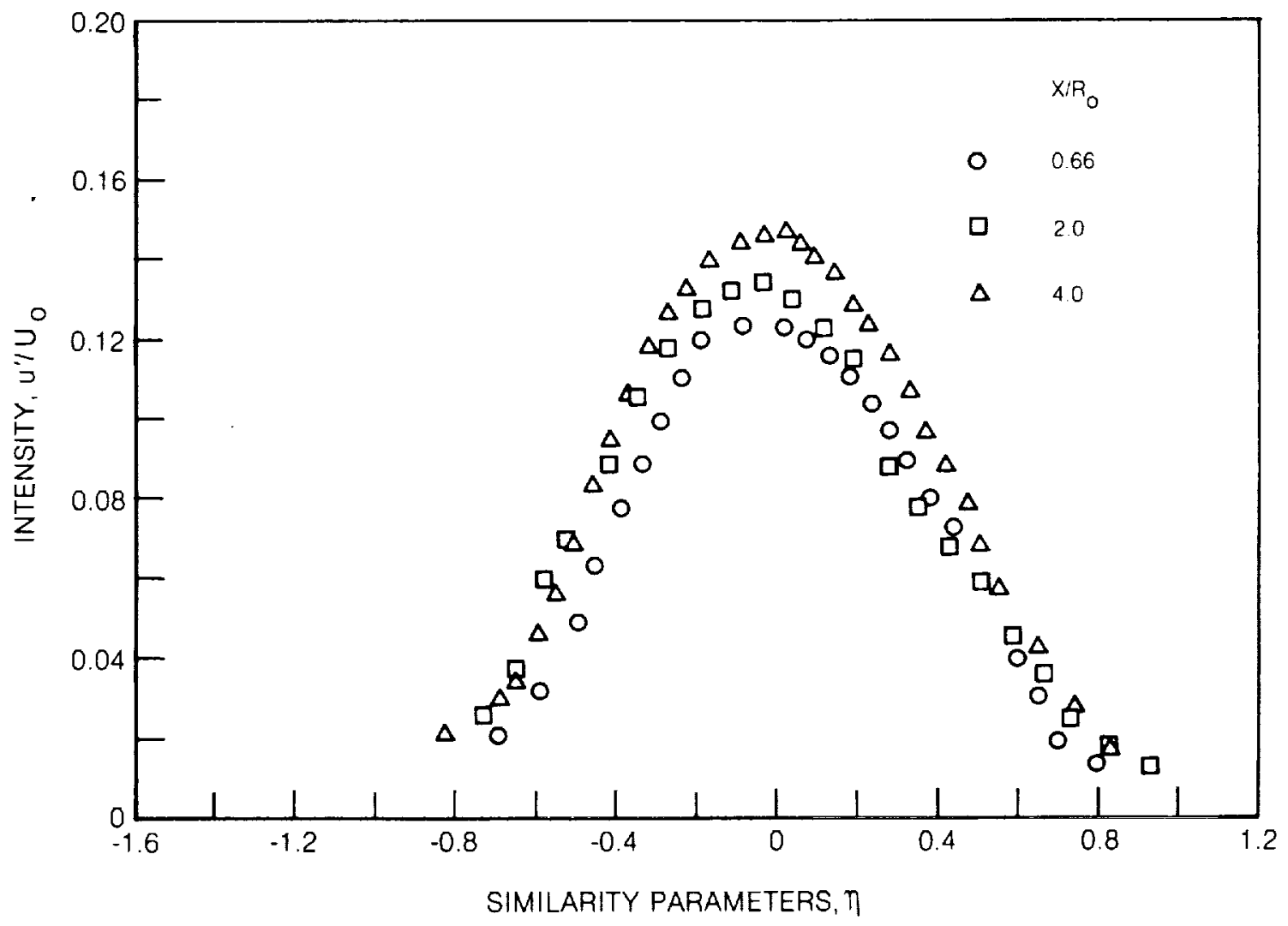

Figure 19 - Similarity of Turbulence Intensity Profiles at Various Axial Stations, $M=0.4$, Tabs Removed. 


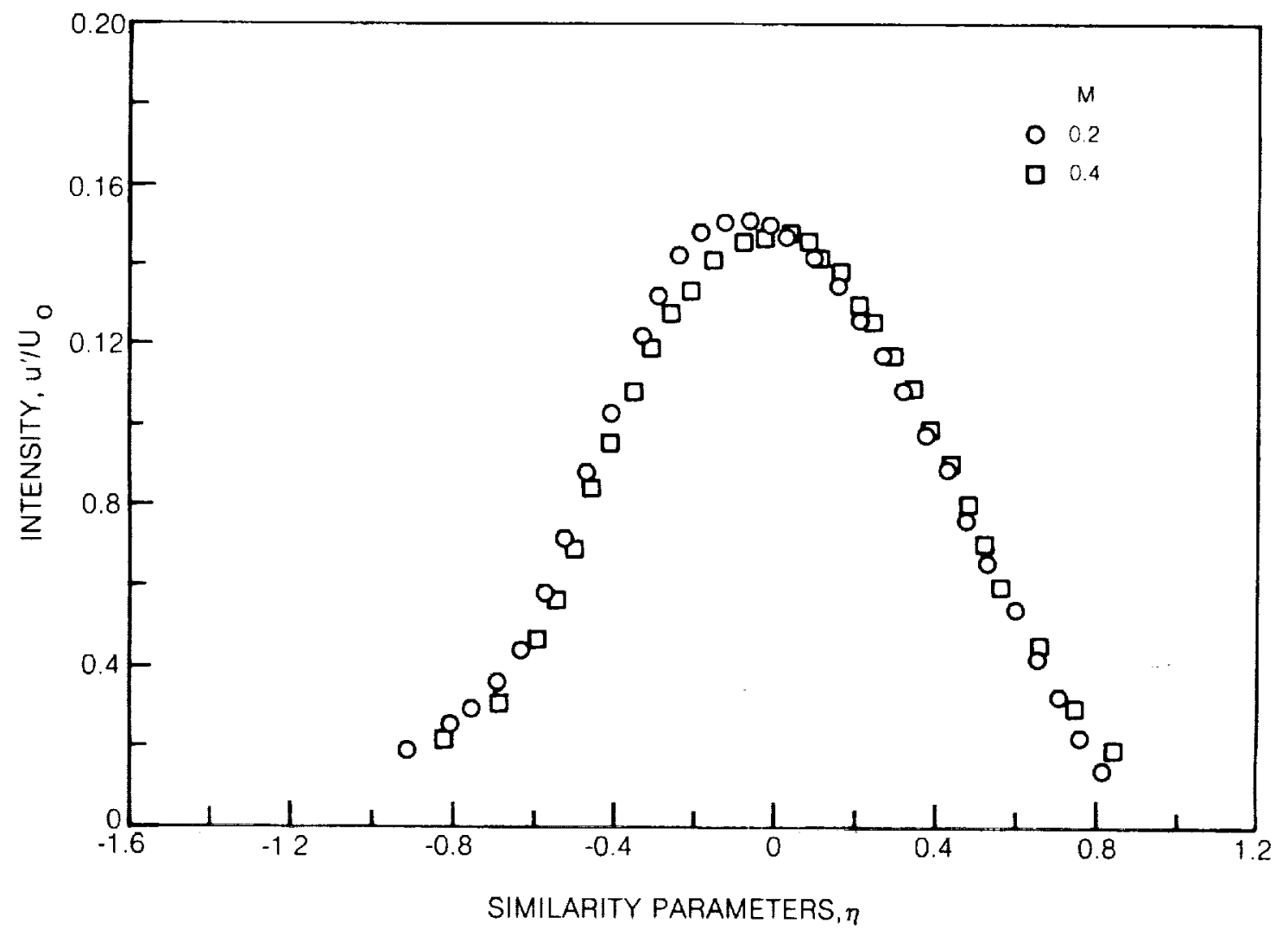

Figure 20 - Similarity of Turbulence Intensity Profiles at Different Mach Numbers, $X / R_{0}=4.0$, Tabs Removed. 


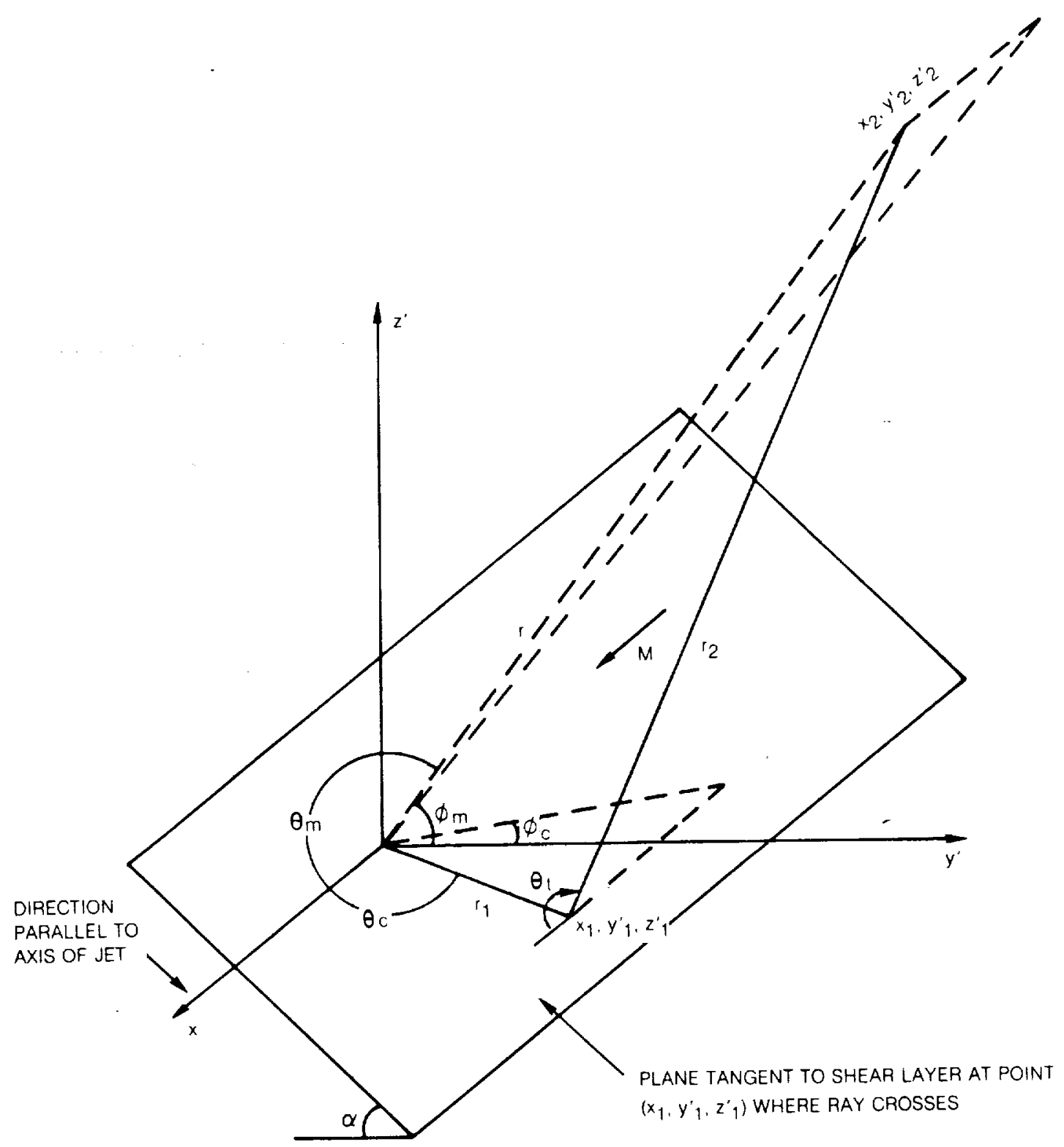

Figure 21 - Geometry for Theoretical Model. 


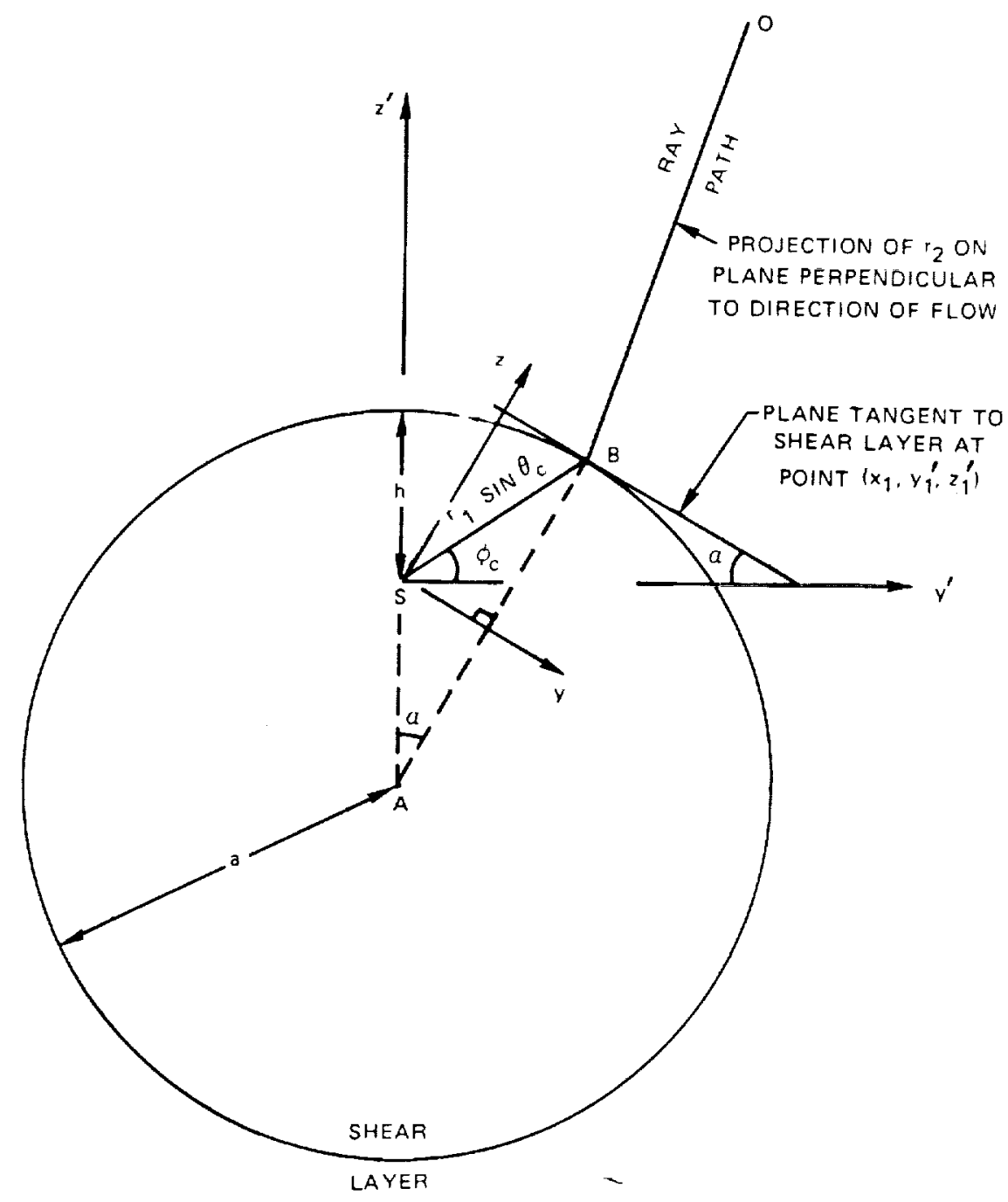

Figure 22 - Cross-Sectional Geometry for Theoretical Model. 


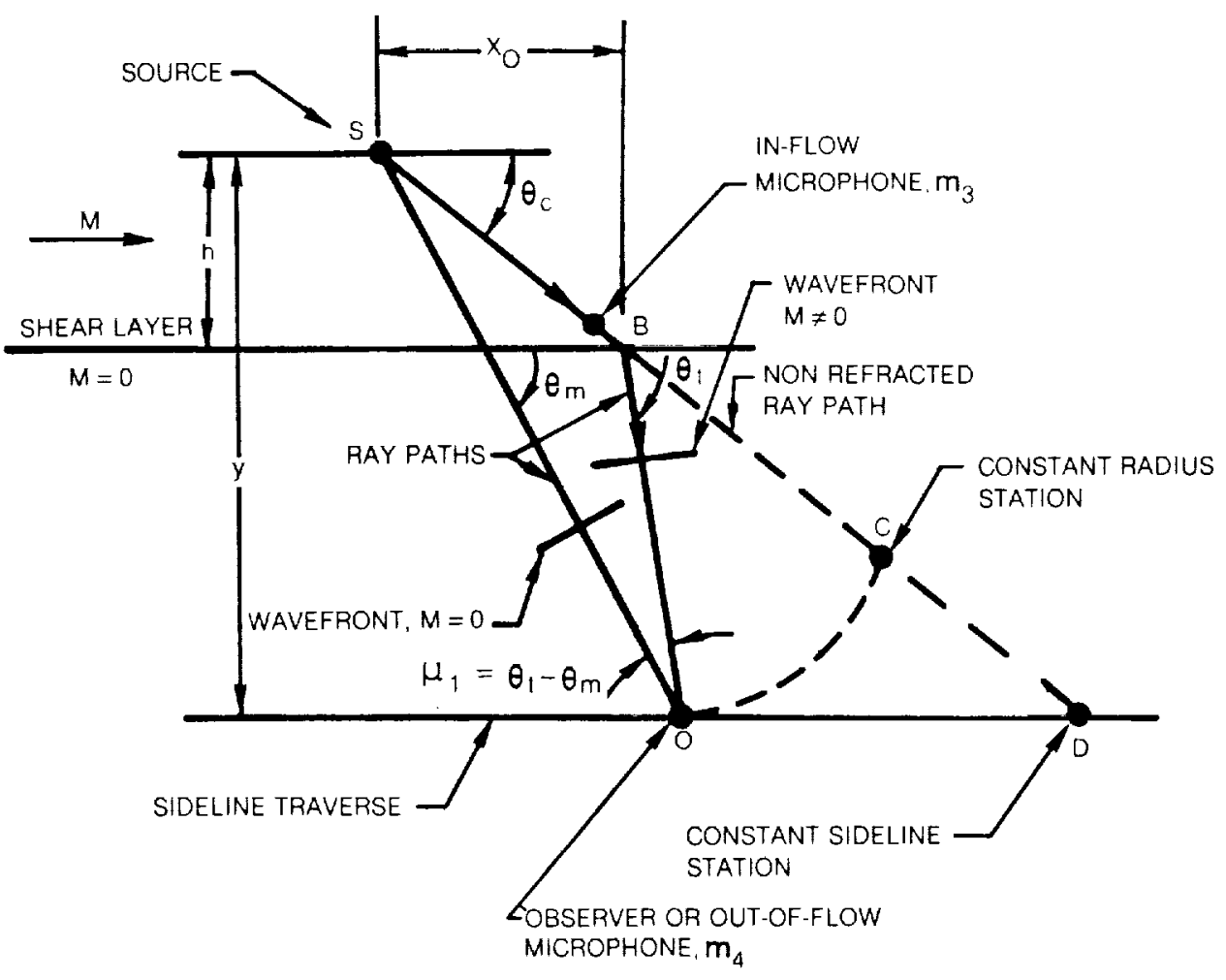

Figure 23 - Geometry for Transmission of Sound Through a Shear Layer. 


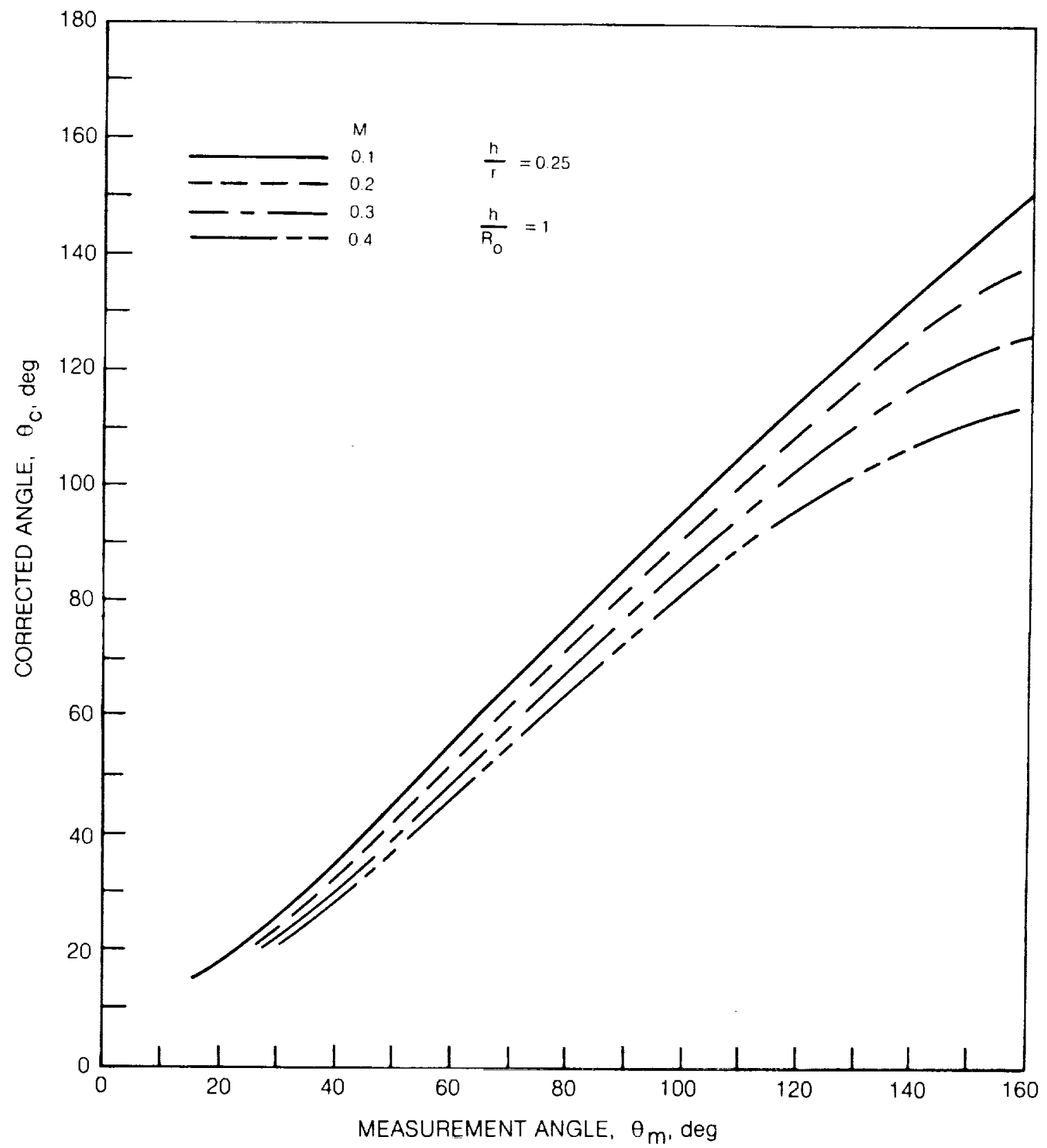

Figure 24 - Theoretical Refraction Angle Correction as a Function of Mach Number, on Axis Acoustic Source Location, Constant Radius Microphone Geometry. 


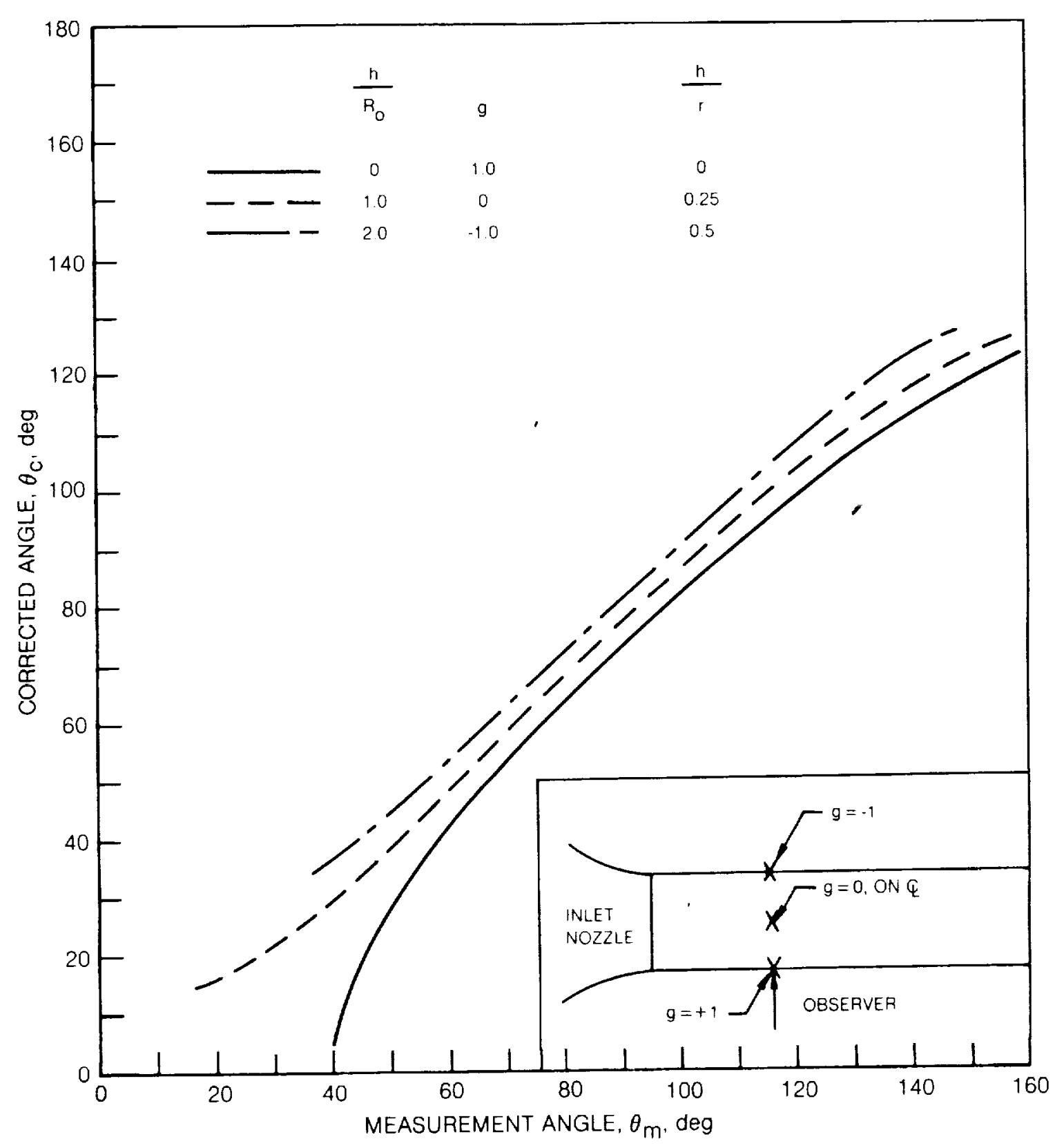

Figure 25 - Theoretical Refraction Angle Correction as a Function of Off-Axis Source Position for $M=0.3$, Constant Radius Microphone Geometry. 


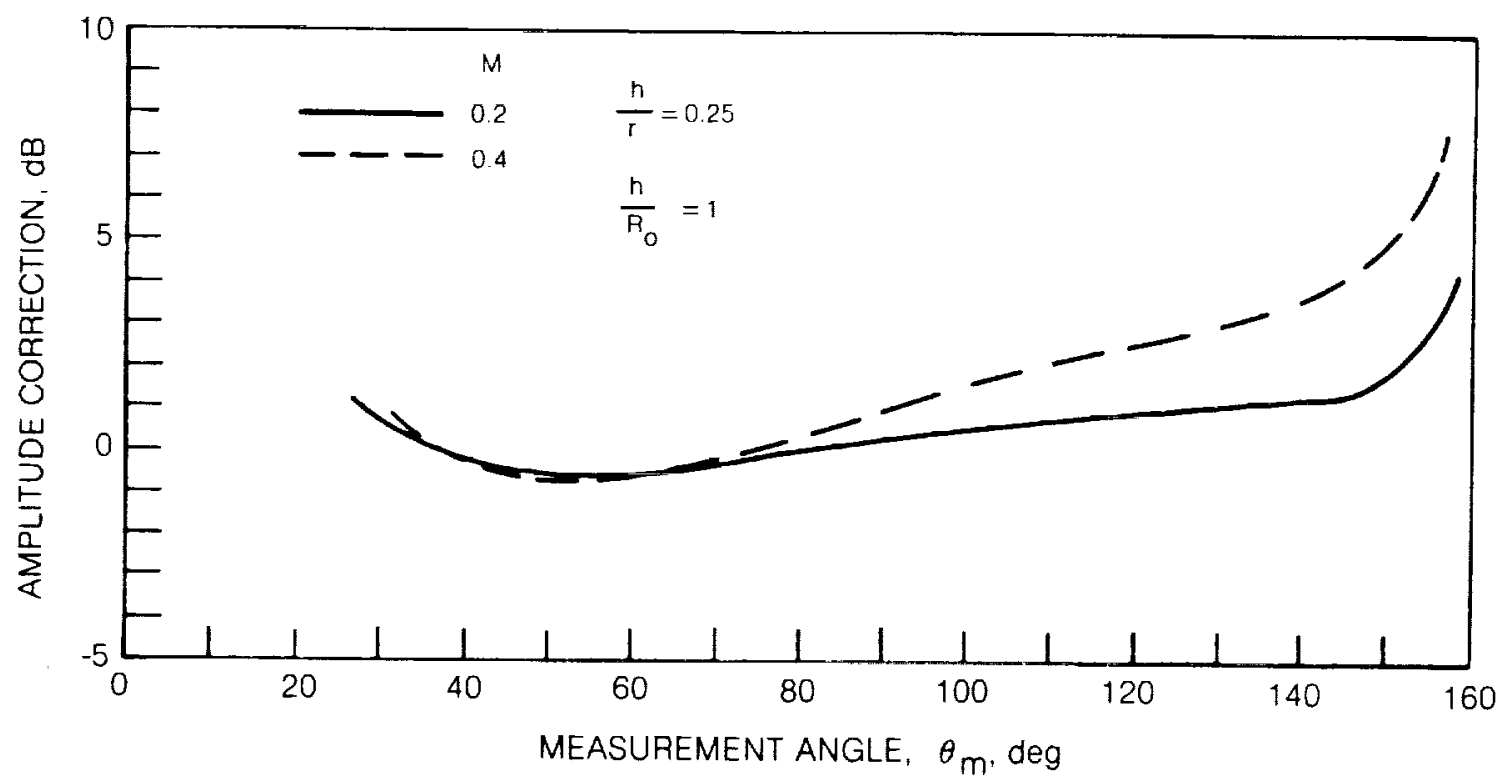

Figure 26 - Theoretical Refraction tmplitude Correction as a Function of Mach Number on Axis Source Location, Constant Radius Microphone Geometry. 


\section{-}

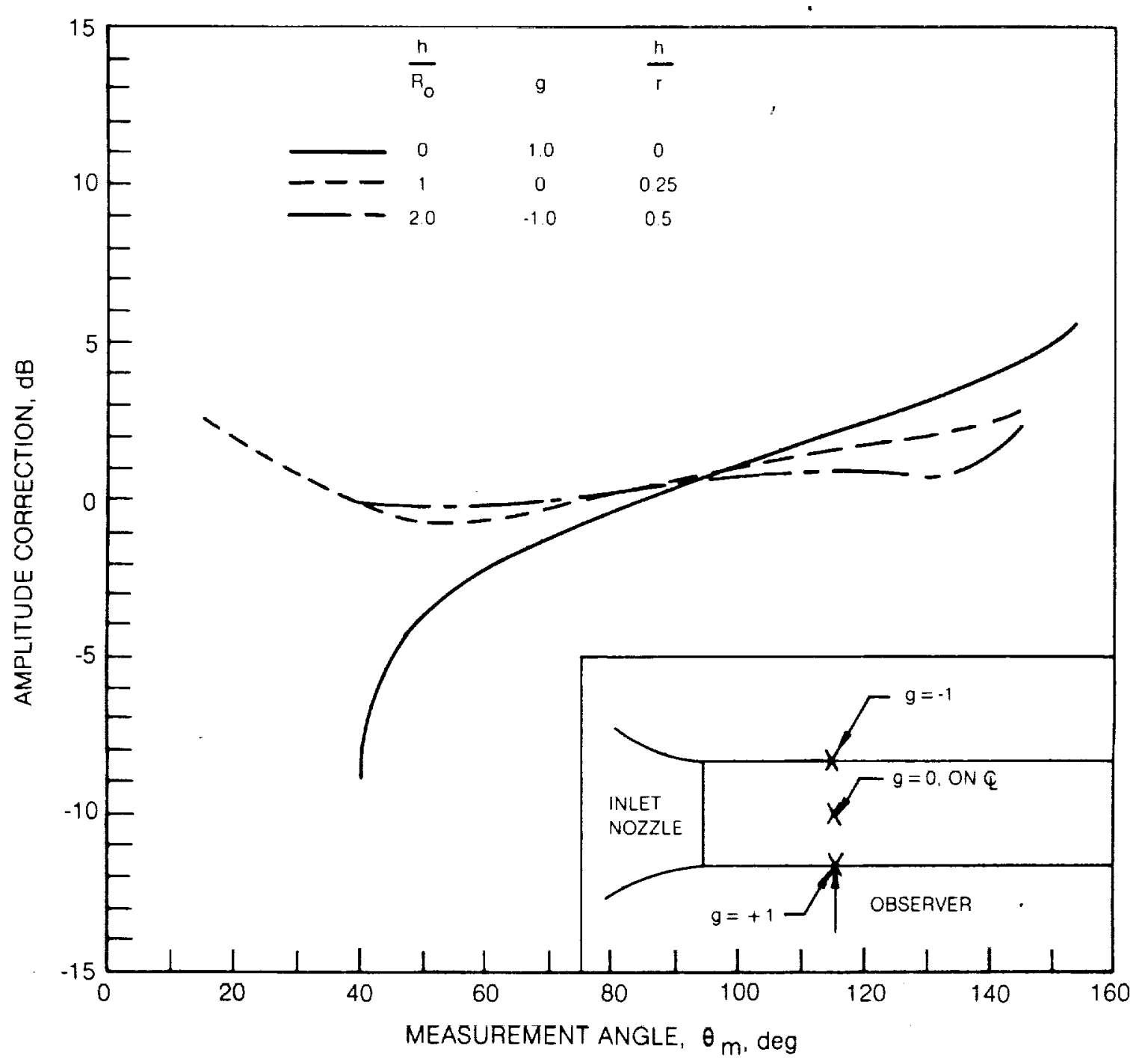

Figure 27 - Theoretical Refraction Amplitude Correction as a Function of Off-Axis Source Position for $\mathbf{M}=\mathbf{0 . 3}$, Constant Radius Microphone Geometry. 


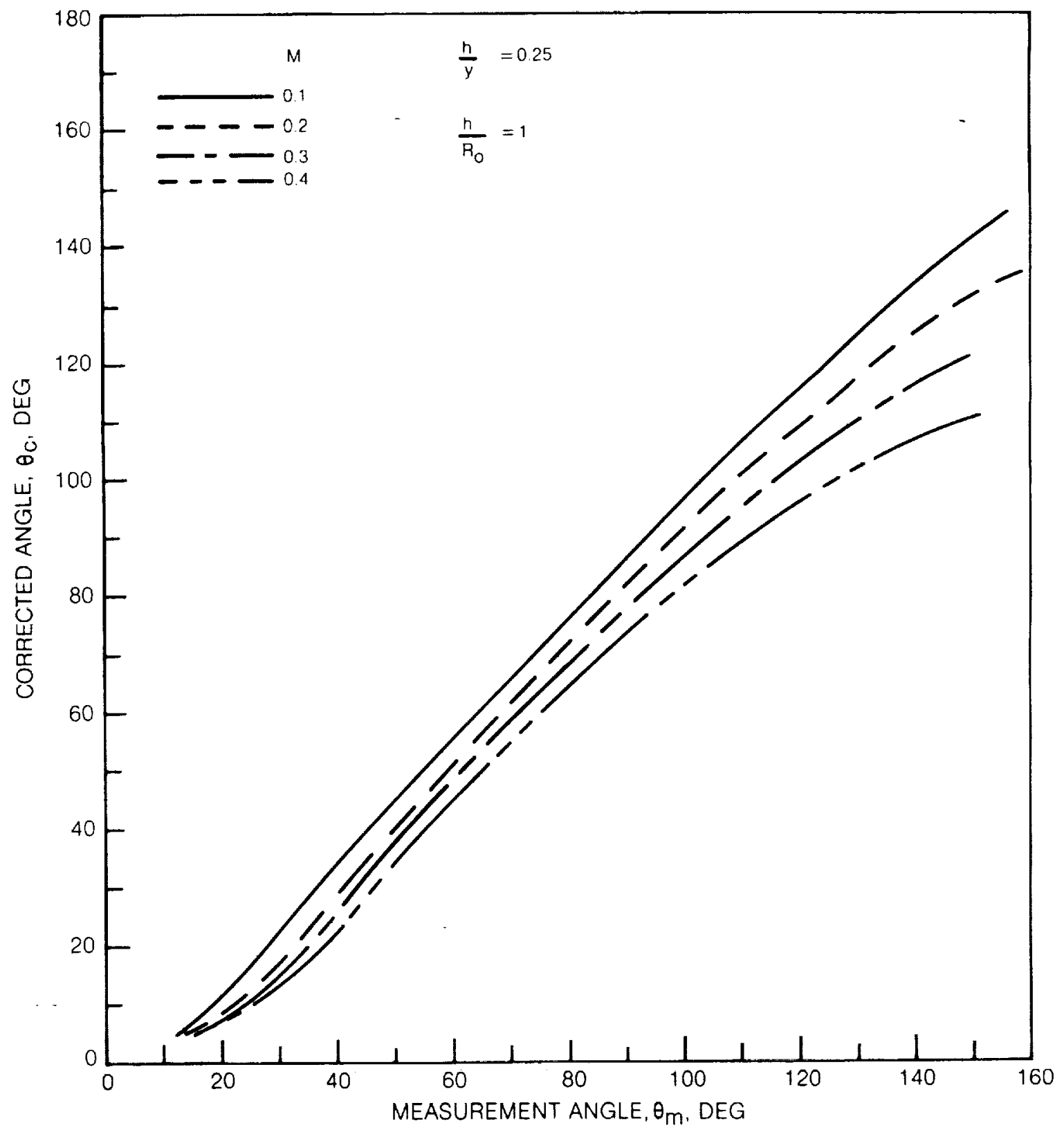

Figure 28 - Theoretical Refraction Angle Correction as a Function of Mach Number, On-Axis Acoustic Source Location, Sideline Microphone Geometry. 


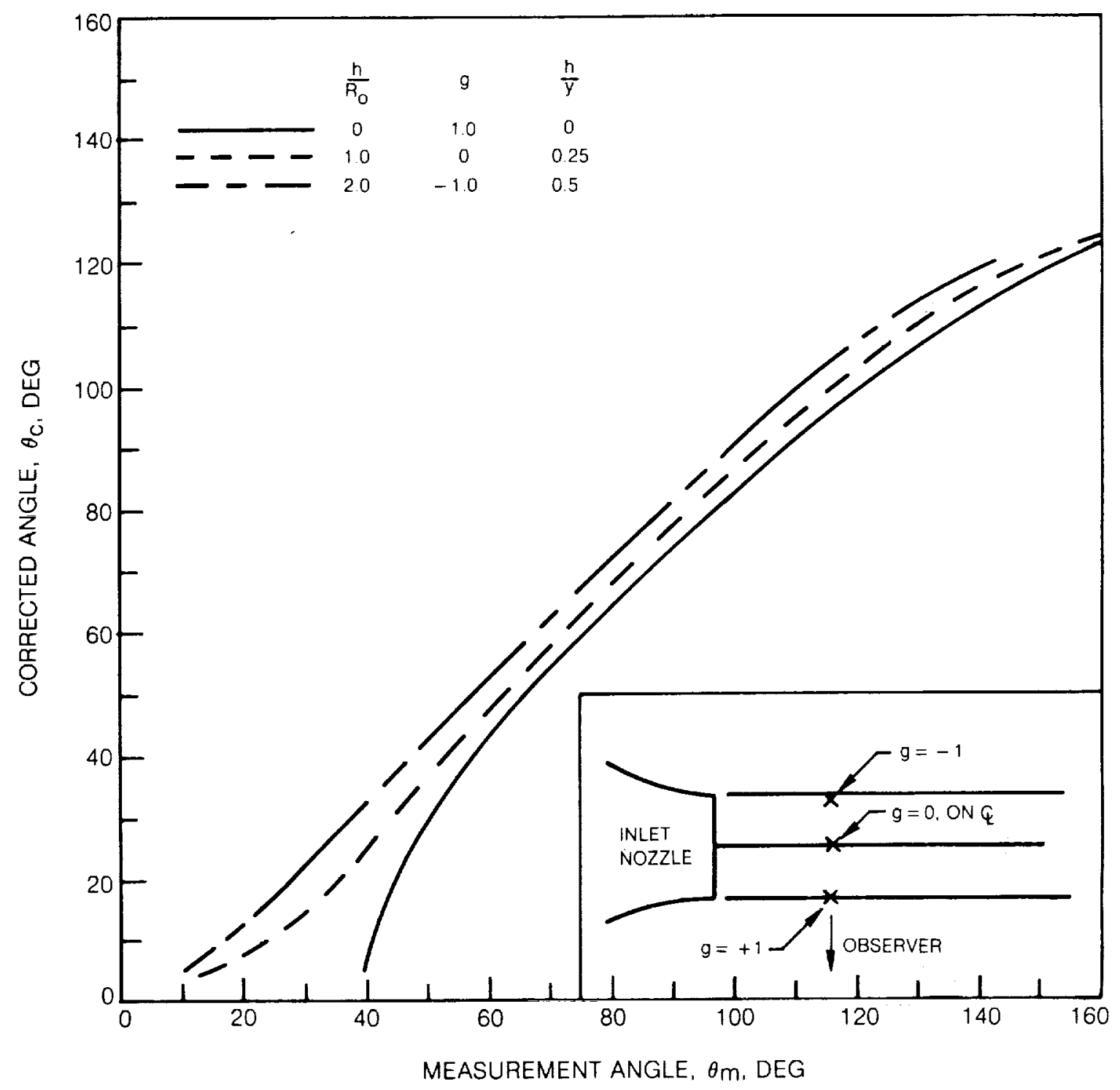

Figure 29 - Theoretical Refraction Angle Correction as a Function of Off.Axis Source Position for $M=0.3$, Sideline Microphone Geometry. 


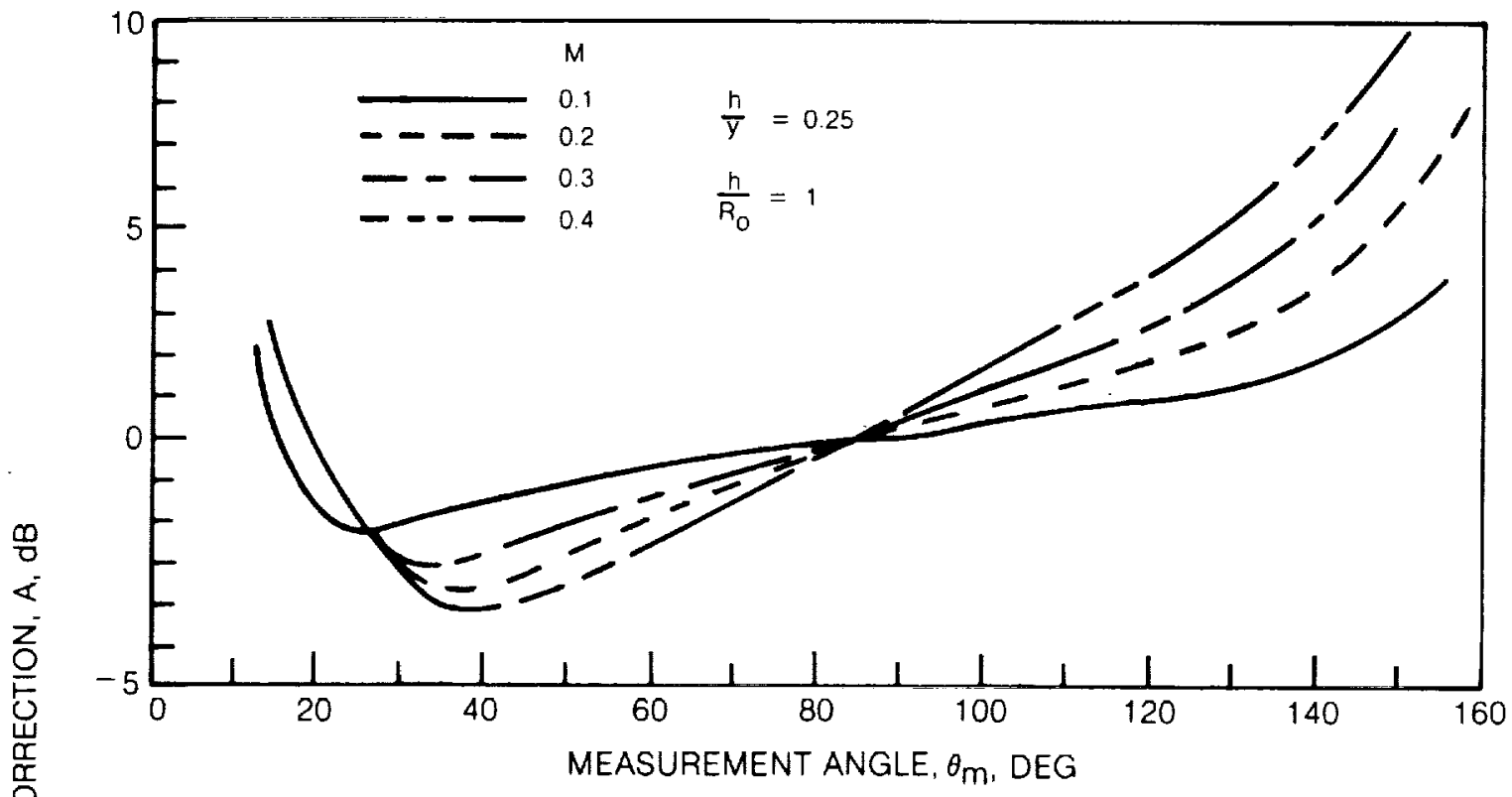

a) AMPLITUDE CORRECTION VERSUS $\theta \mathrm{m}$

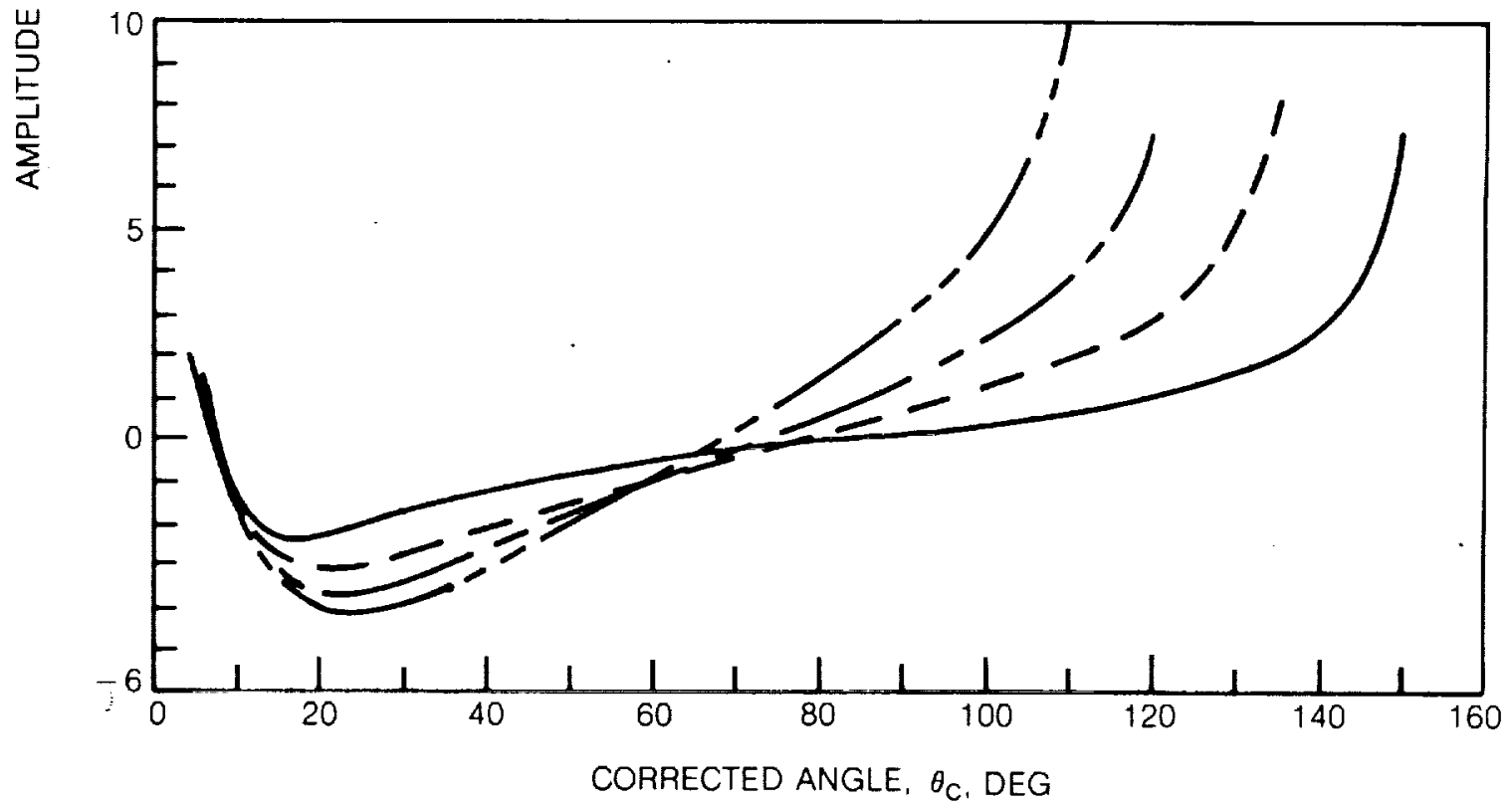

b) AMPLITUDE CORRECTION VERSUS $\theta_{C}$

Figure 30 - Theoretical Refraction Amplitude Correction as a Function of Mach Number, on Axis Acoustic Source Location. 


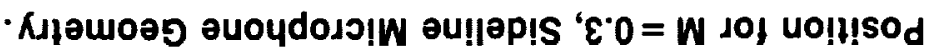

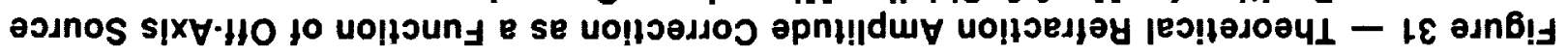

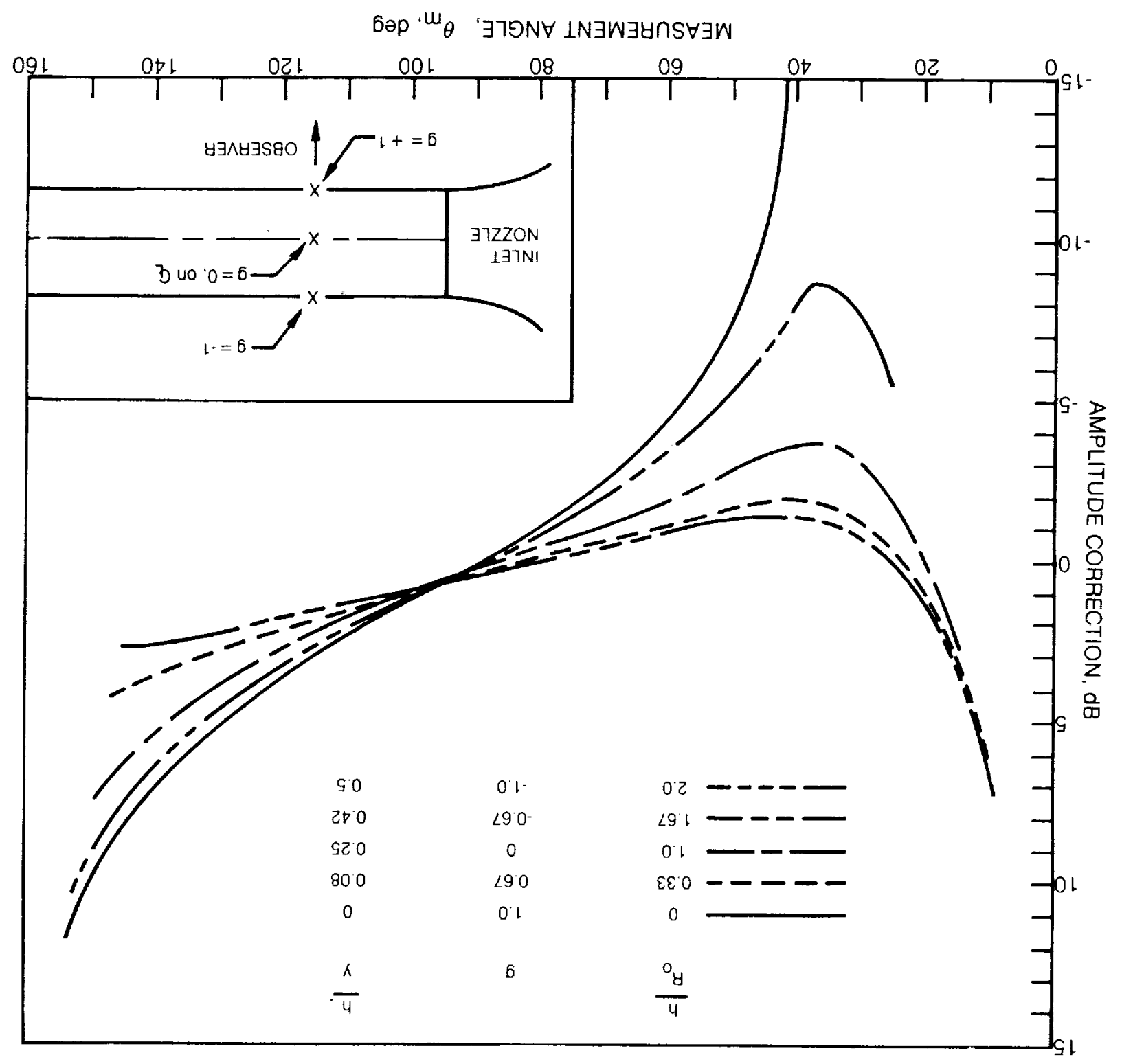




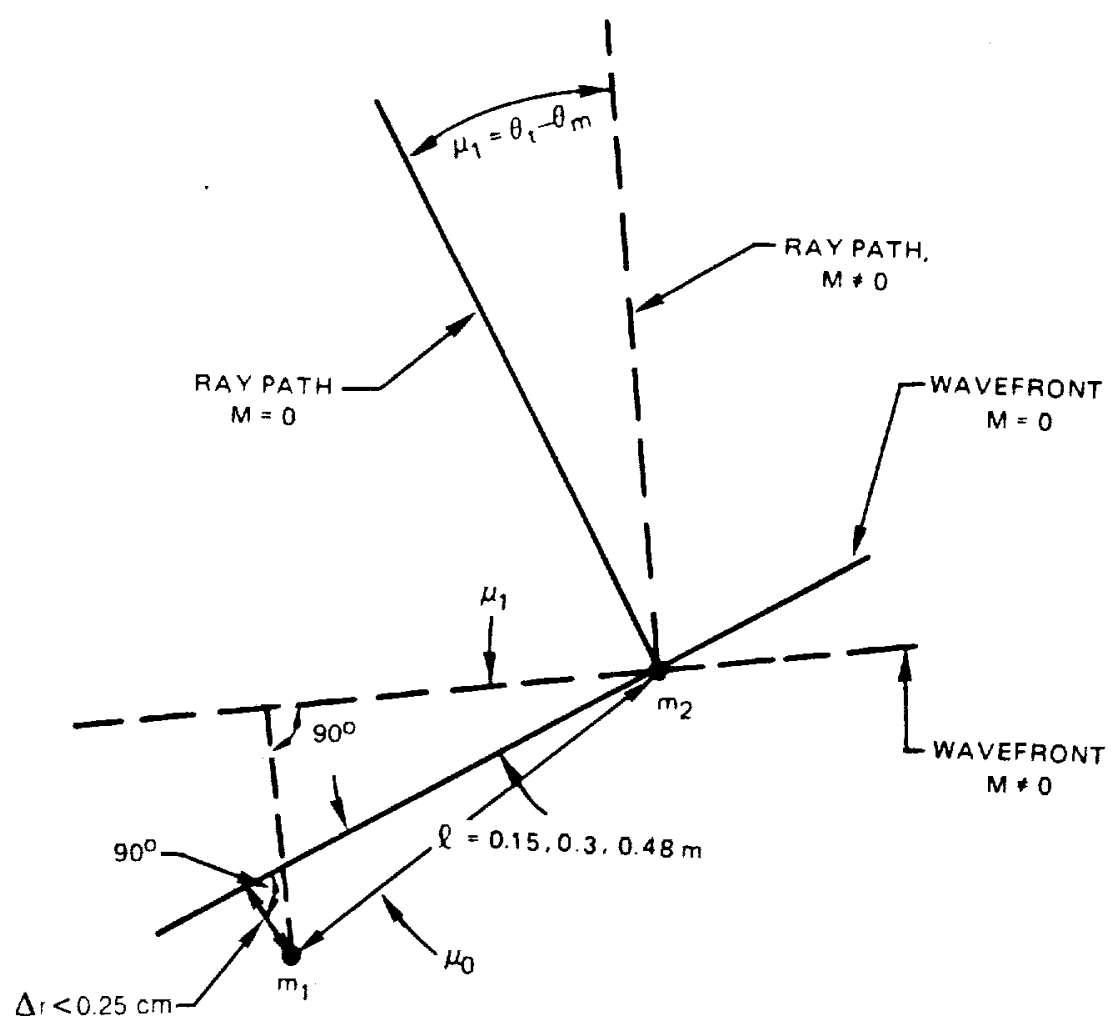

Figure 32 - Model of Wavefront Angle Changes at Far-Field Microphone Location. 


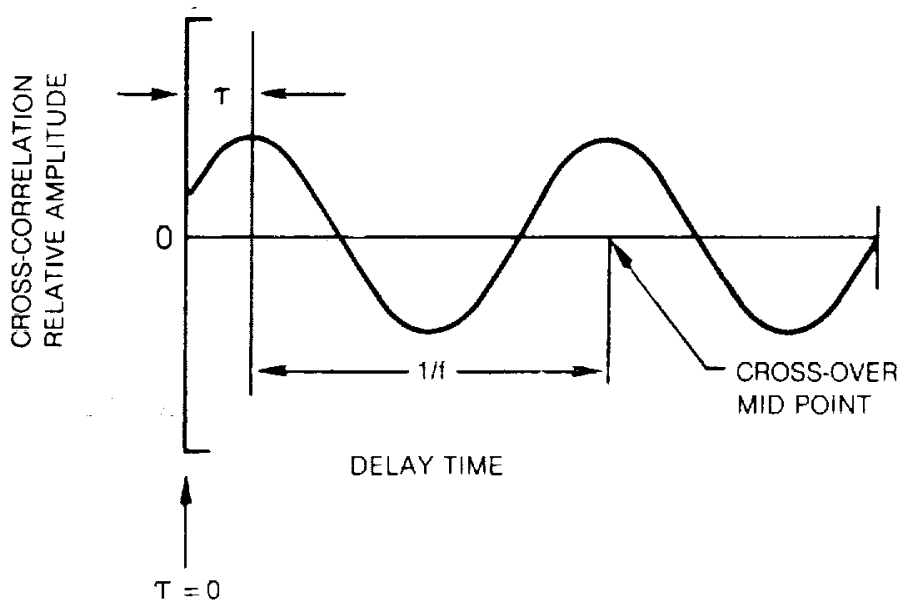

Figure 33 - Two Microphone Cross-Correlation Function at $M=0$. 


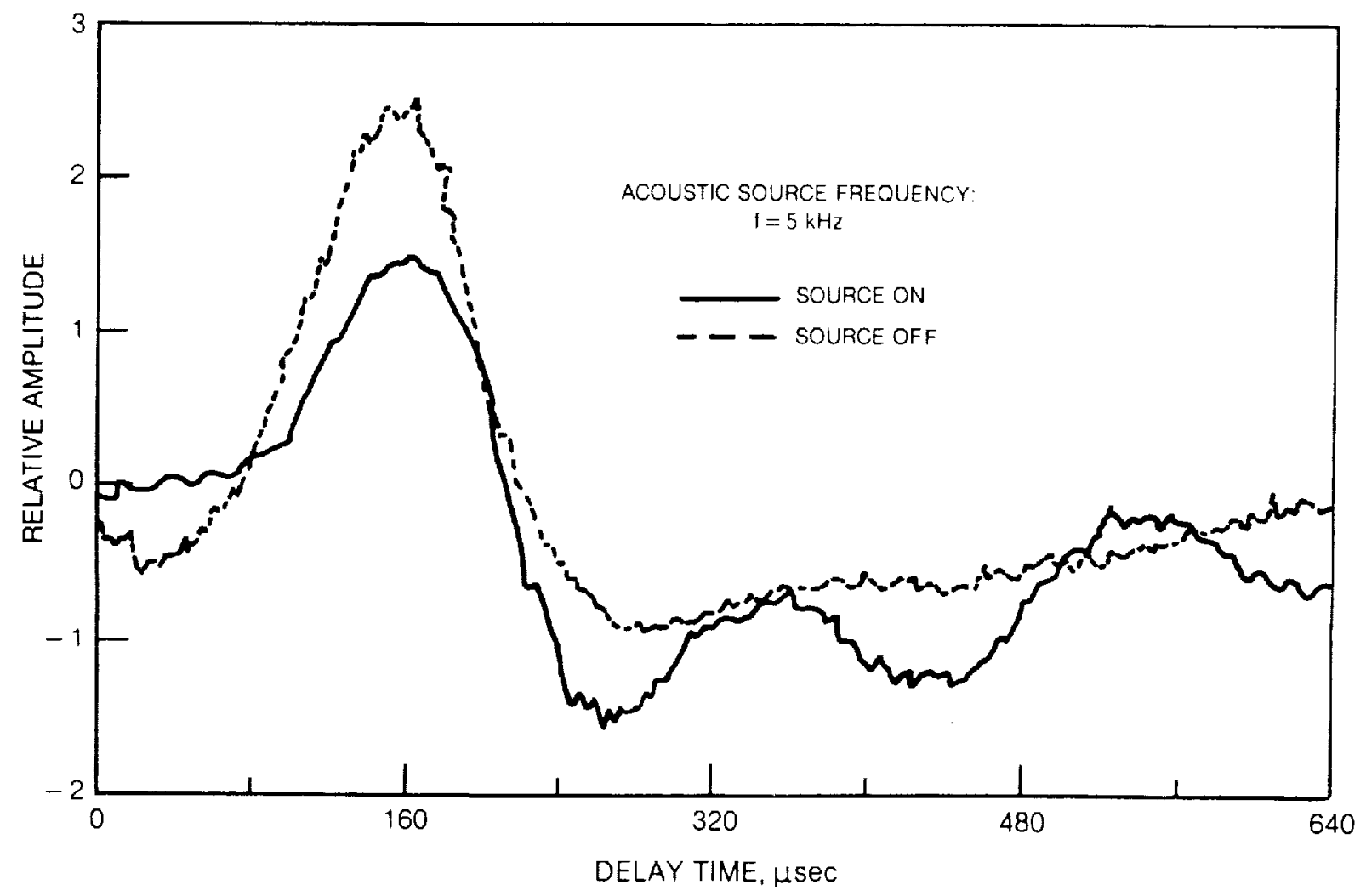

Figure 34 - Two Microphone Cross-Correlation Function at Mach Number, $M=0.4$, Measurement Angle, $\theta_{m}=37.5^{\circ}$, Test Configuration 1. 


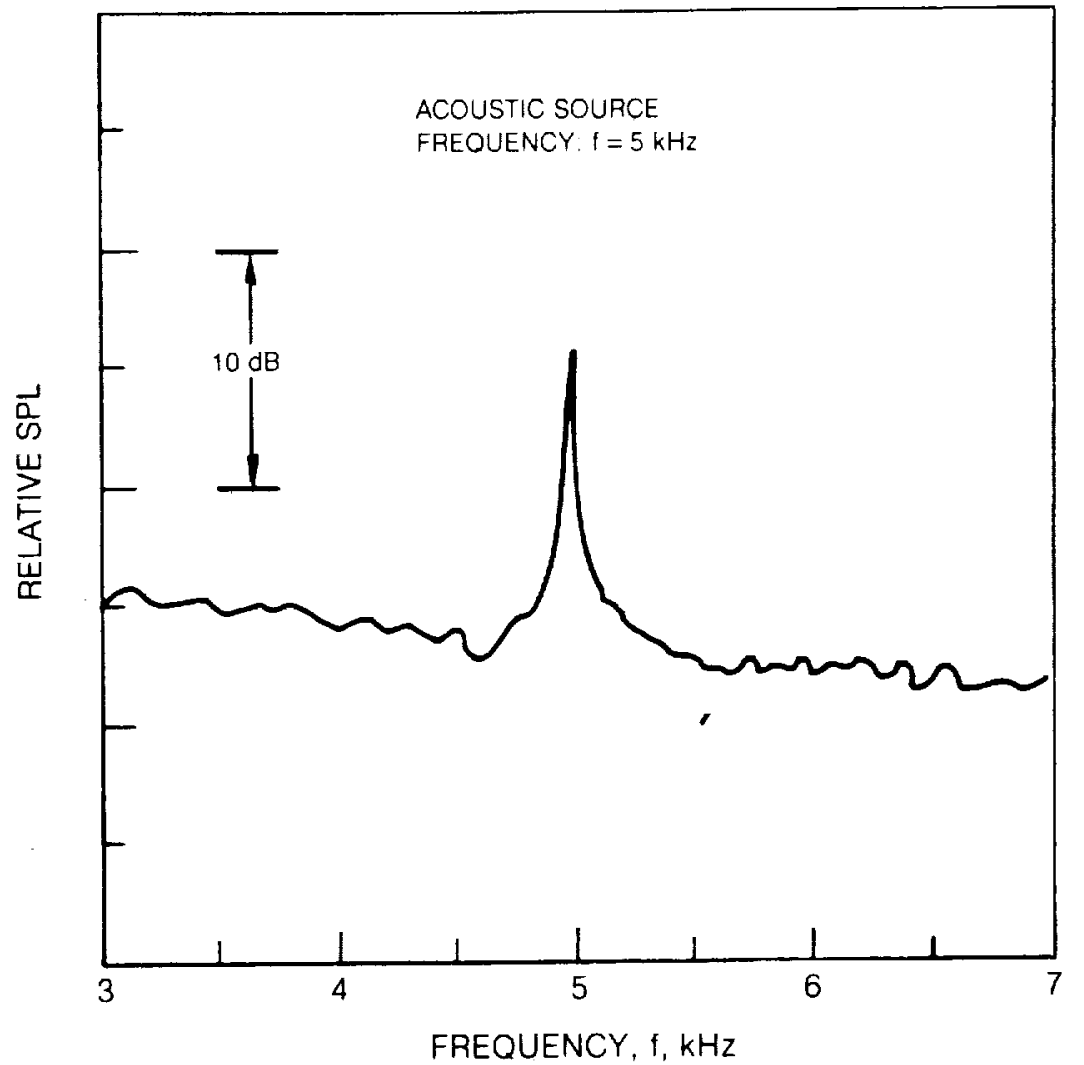

Figure 35 - Typical Far-Field Microphone Spectrum at Mach Number, $M=0.4$, Measurement Angle, $\theta_{m}=37.5^{\circ}$, Test Configuration 1. 


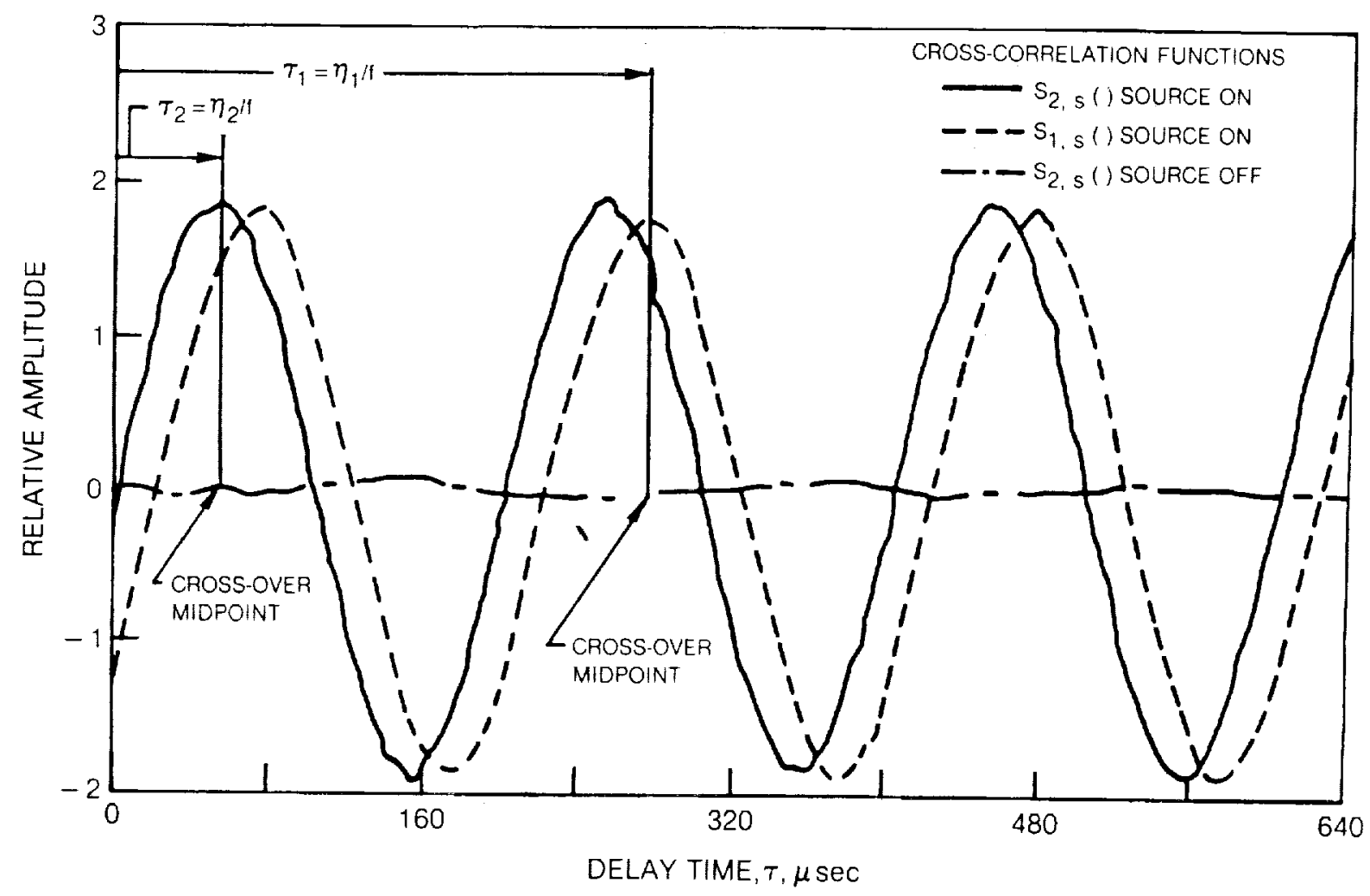

Figure 36 - Microphone-Signal Generator Cross-Correlation at Mach Number, $M=0.4$, Frequency, $f=10 \mathrm{kHz}$ Measurement Angle, $\theta_{\mathbf{m}}=37.5^{\circ}$, Test Configuration 1 . 


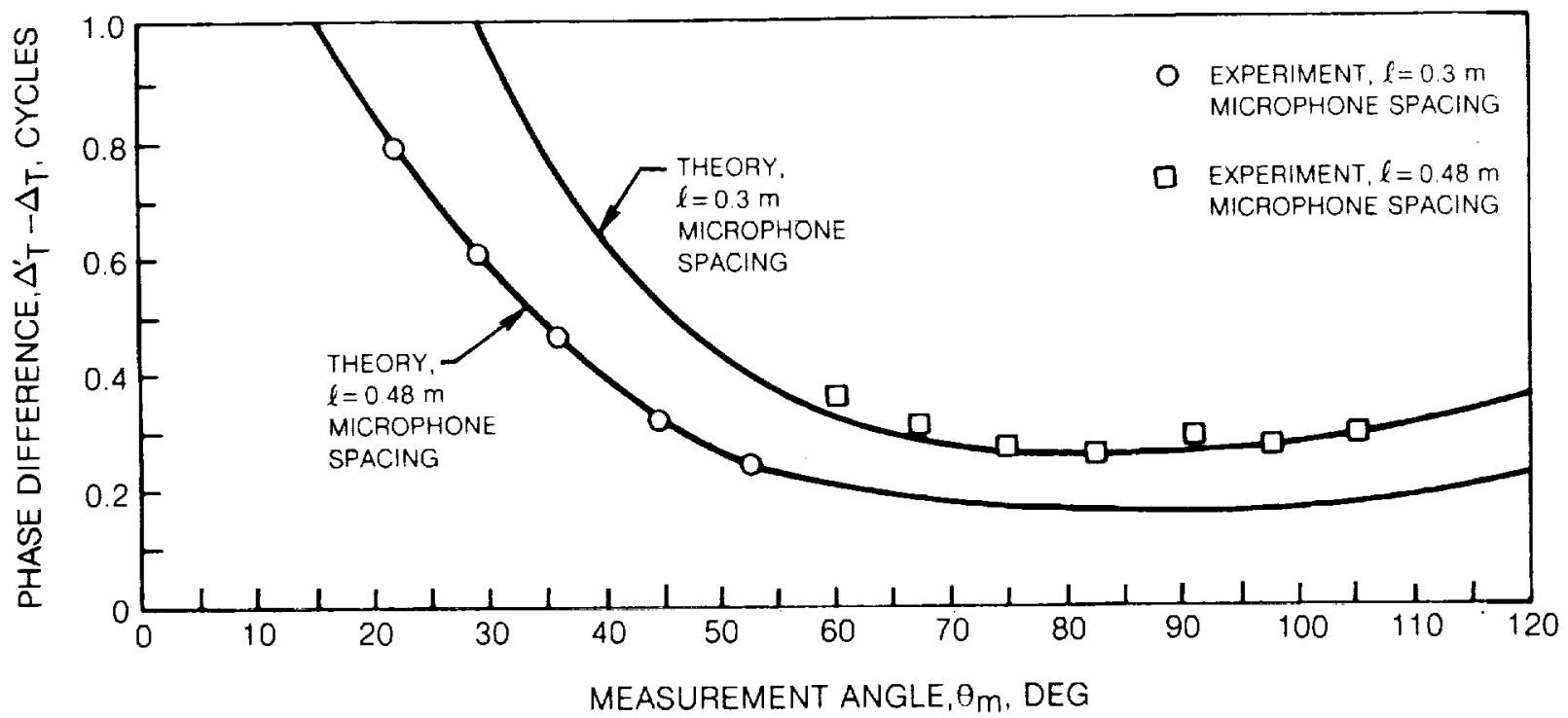

Figure 37 - Comparison of Measured and Theoretical Phase Difference at Mach Number, $M=0.3$, Frequency, $f=2.5 \mathrm{kHz}$, Test Configuration 1. 
$\underset{\ldots}{\varpi}$

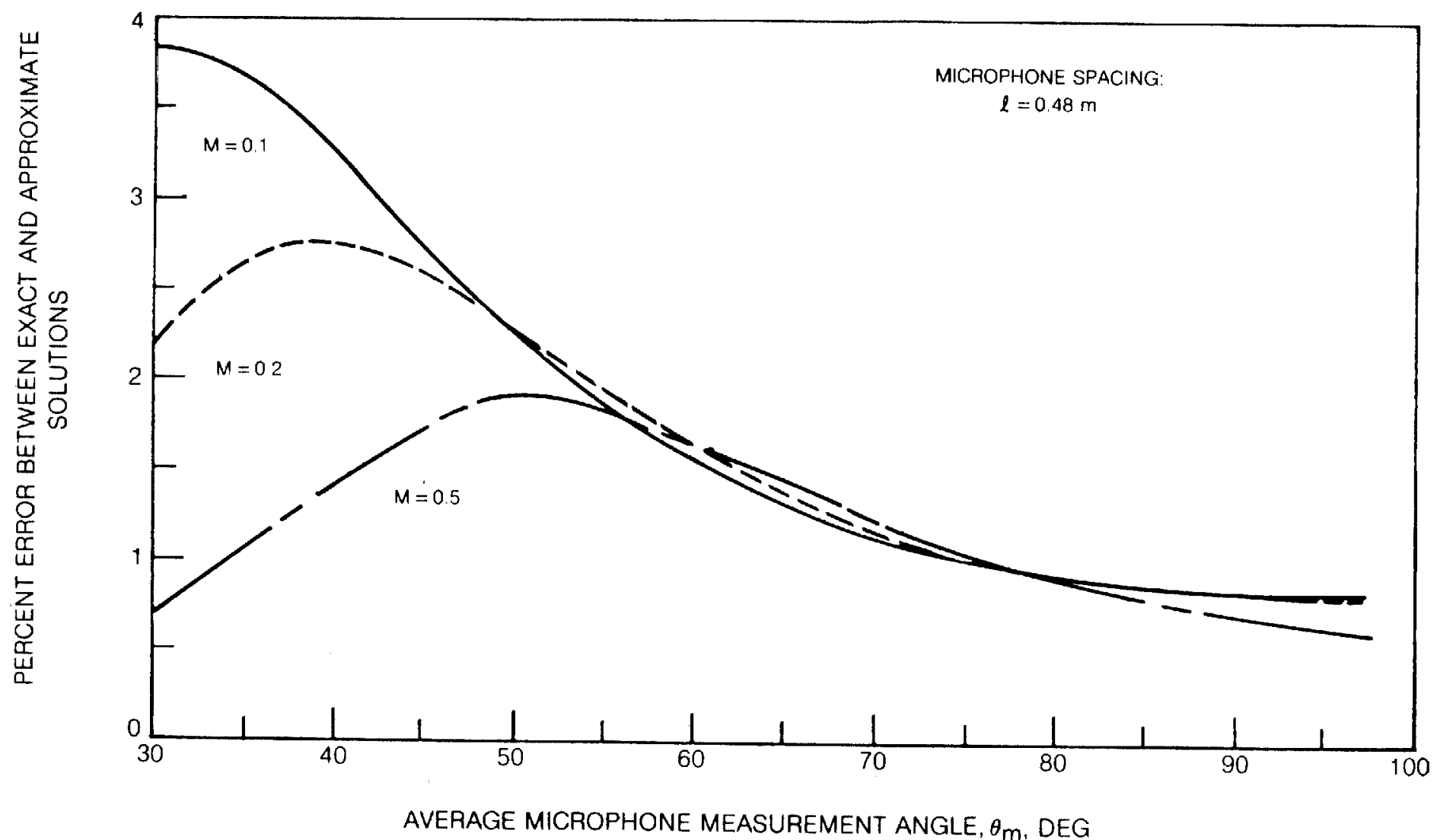

Figure 38 - Assessment of Error Due to Plane-Wave Propagation Assumption. 


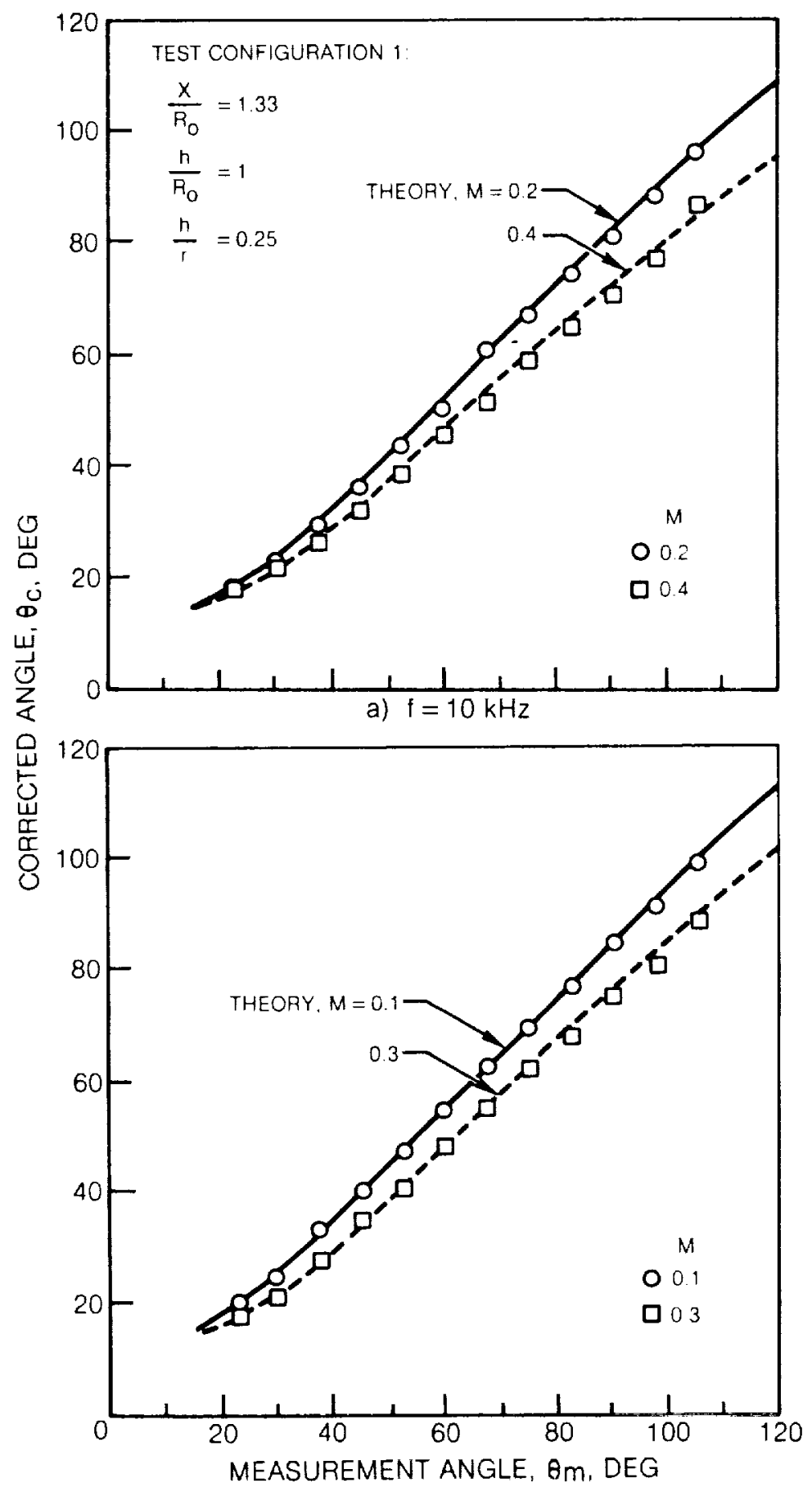

b) $f=10 \mathrm{kHz}$

Figure 39 - Comparison of Measured and Theoretical Refraction Angle Correction as a Function of Mach Number and Frequency, Test Configuration 1. 


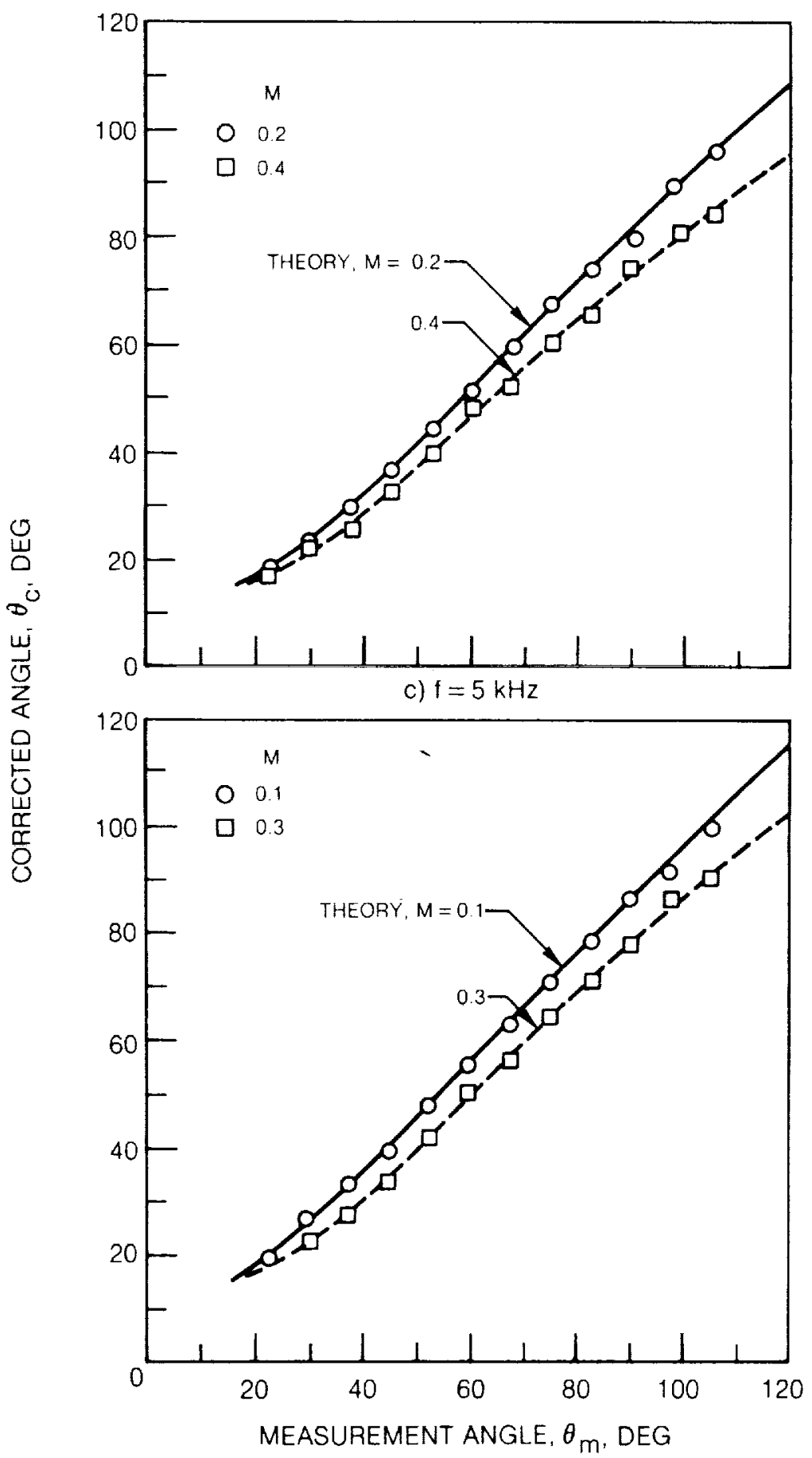

d) $f=5 \mathrm{kHz}$

Figure $39-$ Continued. 


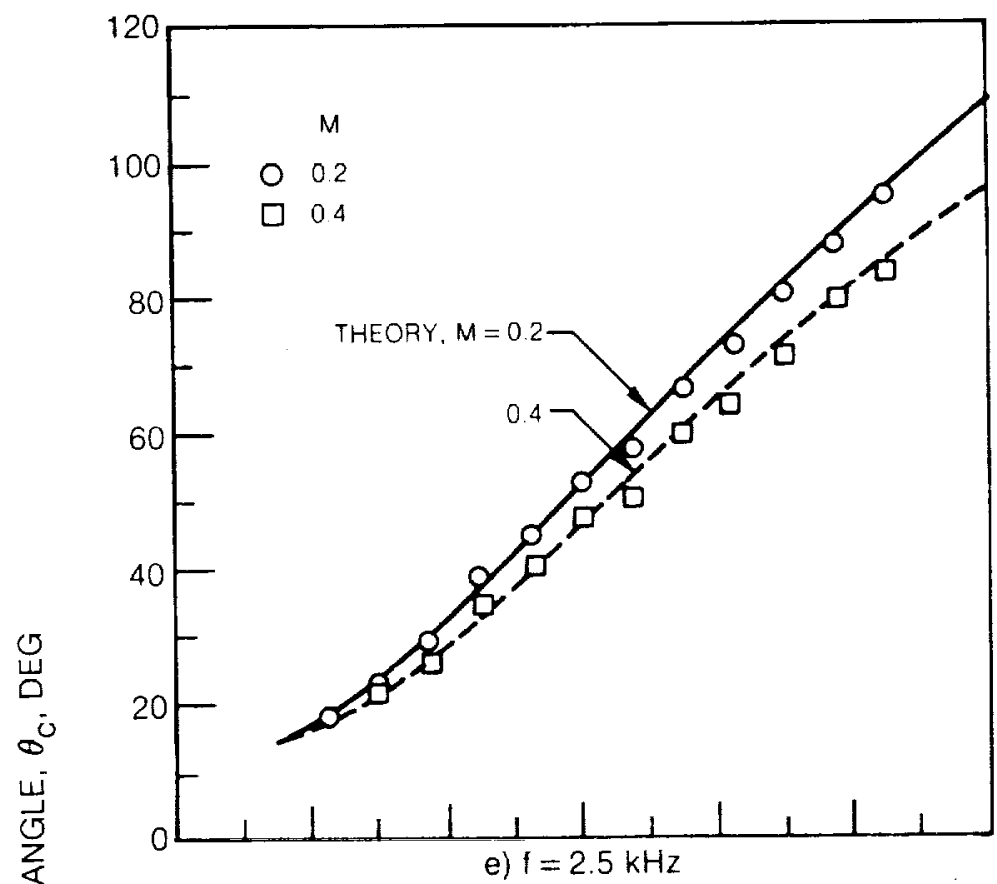

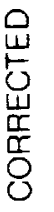

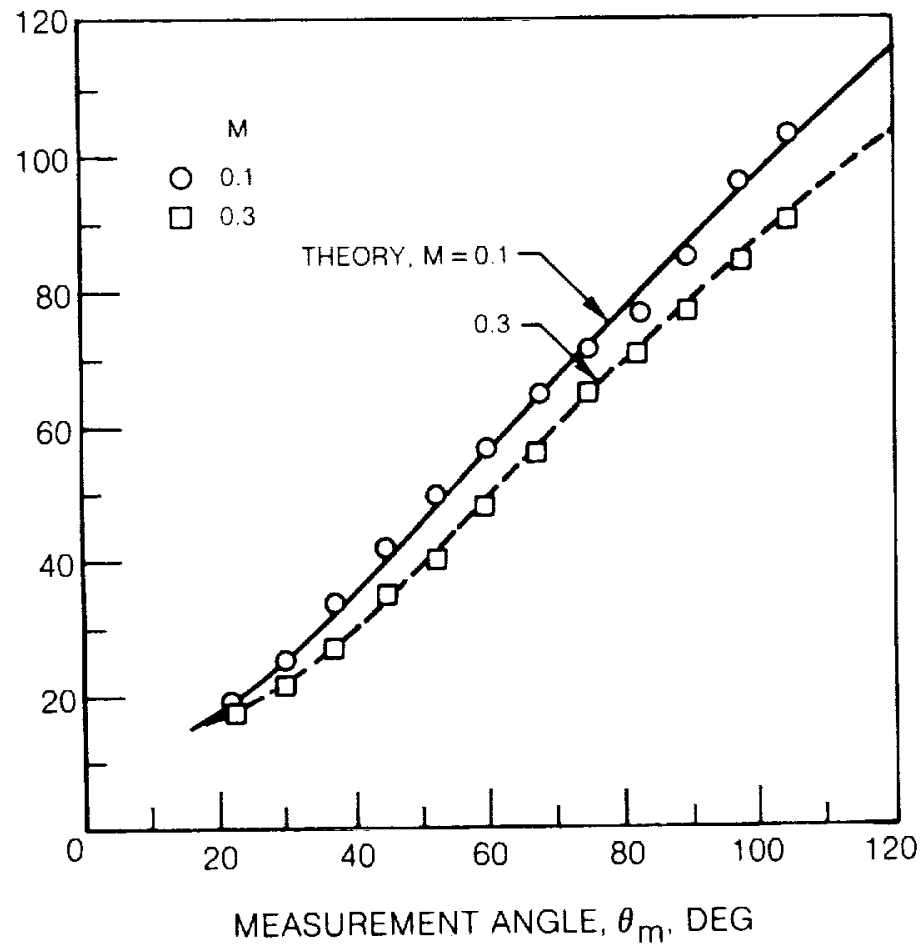

f) $f=2.5 \mathrm{kHz}$

Figure $39-$ Continued. 


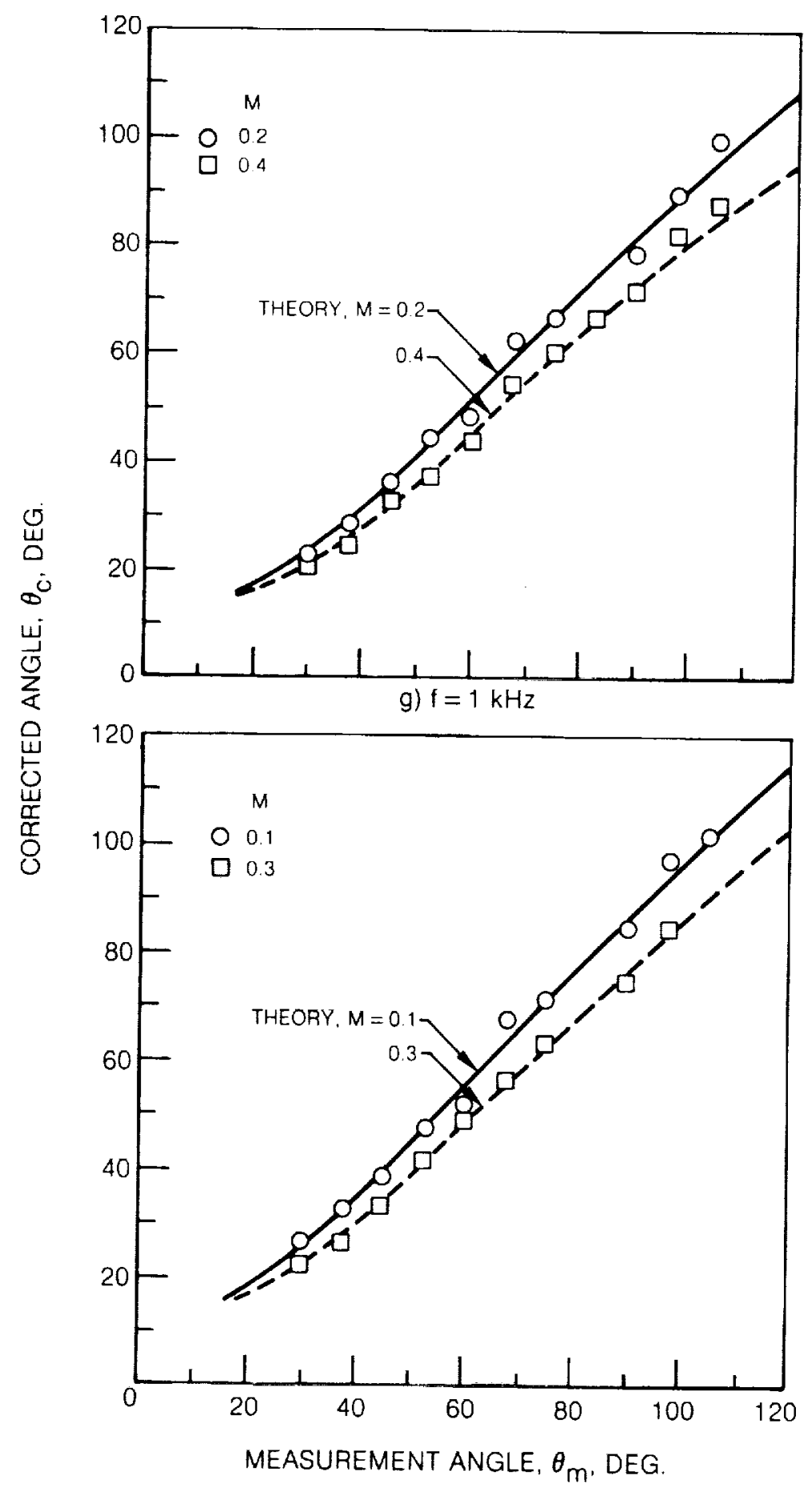

h) $\mathrm{f}=1 \mathrm{kHz}$

Figure 39 - Concluded. 


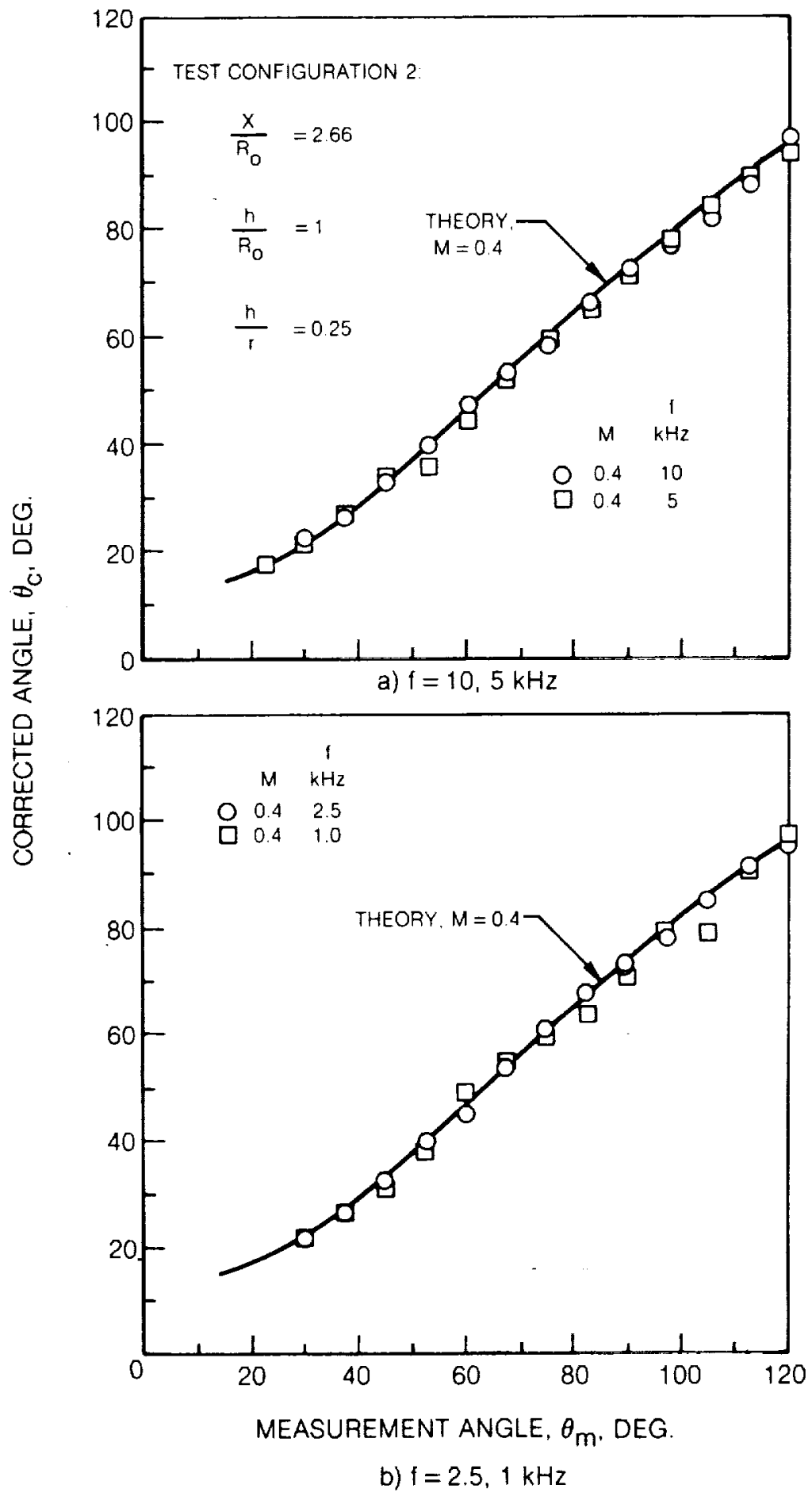

Figure 40 - Comparison of Experimental and Theoretical Refraction Angle Correction as a Function of Mach Number and Frequency,

Test Configuration 2. 


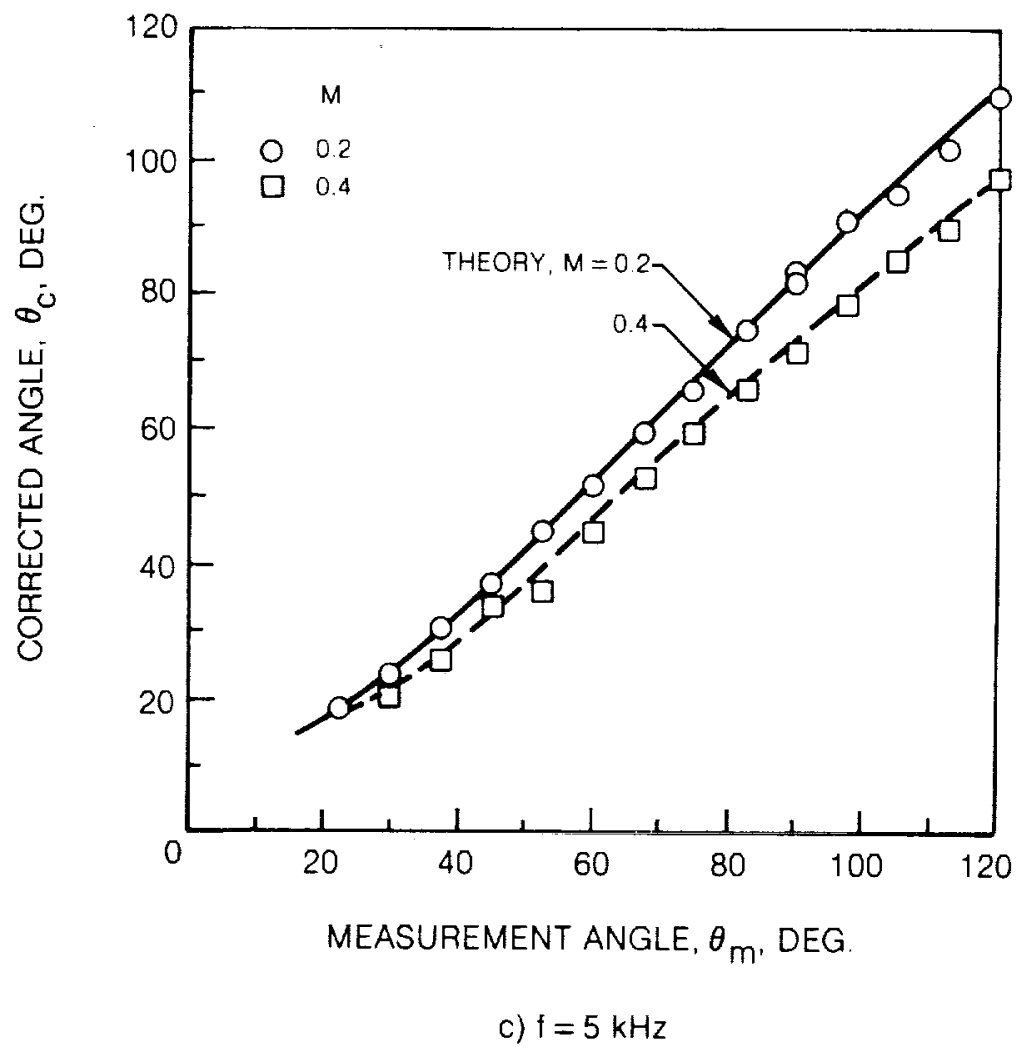

Figure 40 - Concluded. 

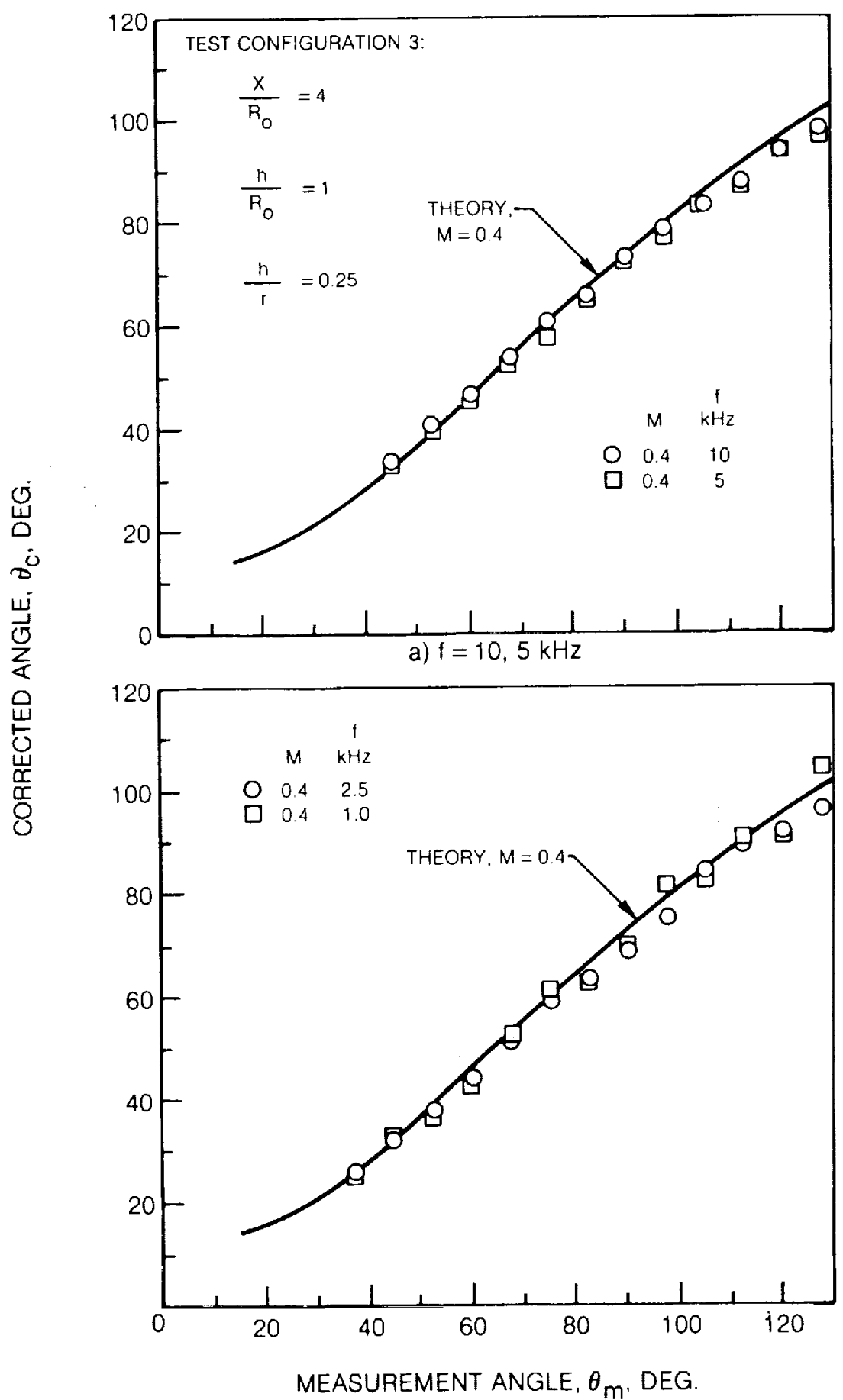

b) $f=2.5,1 \mathrm{kHz}$

Figure 41 - Comparison of Experimental and Theoretical Refraction Angle Correction as a Function of Mach Number and Frequency, Test Configuration 3. 


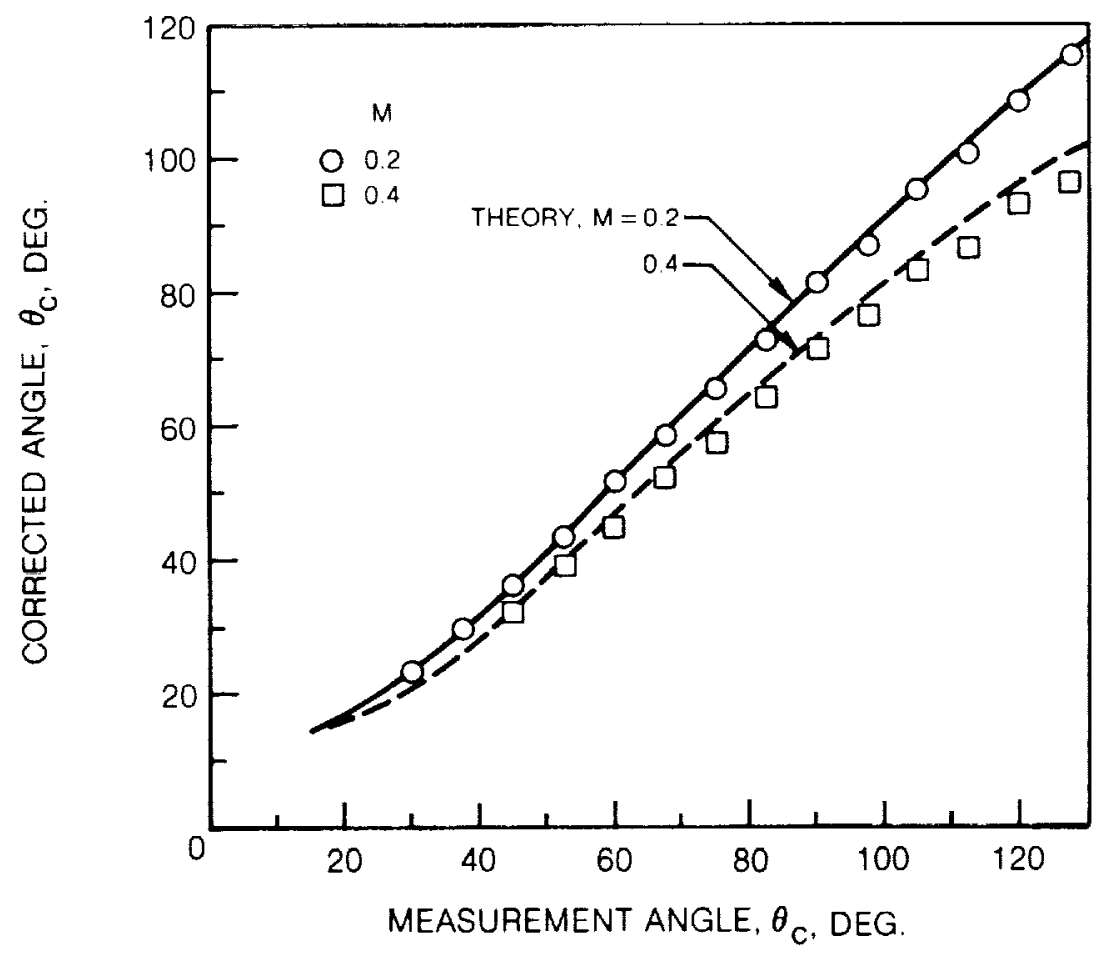

c) $f=5 \mathrm{kHz}$

Figure 41 - Concluded. 


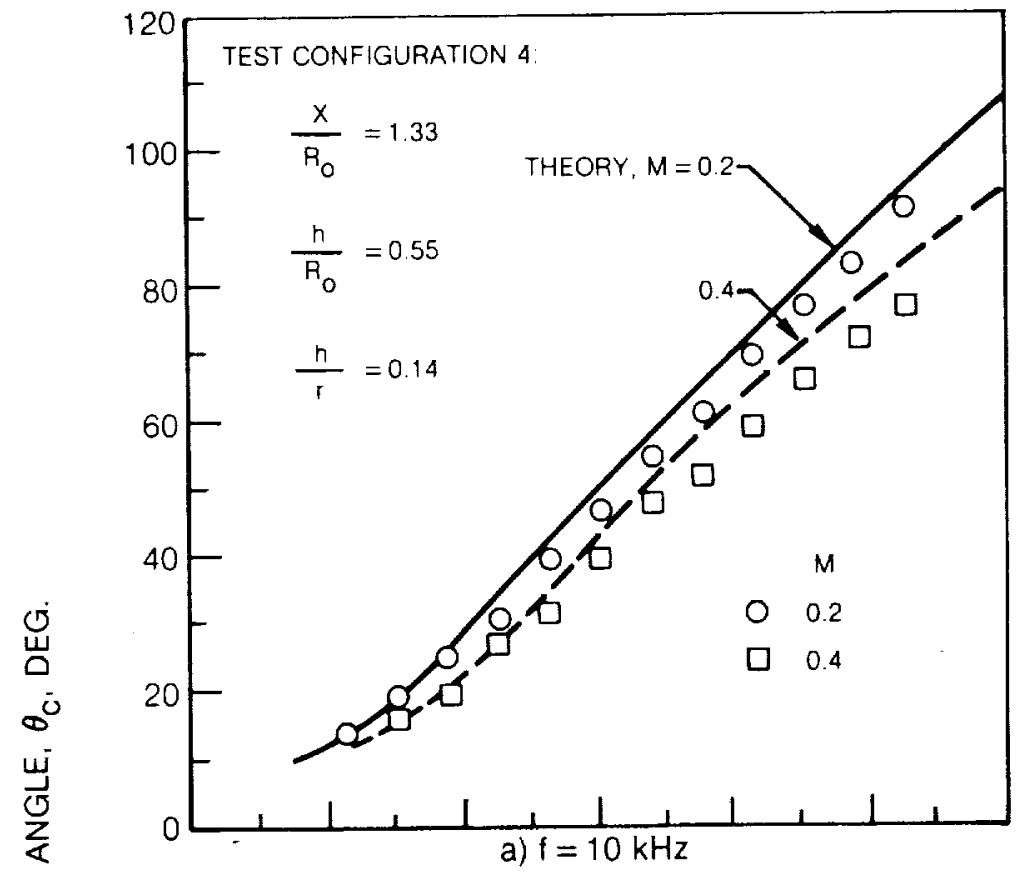

岂

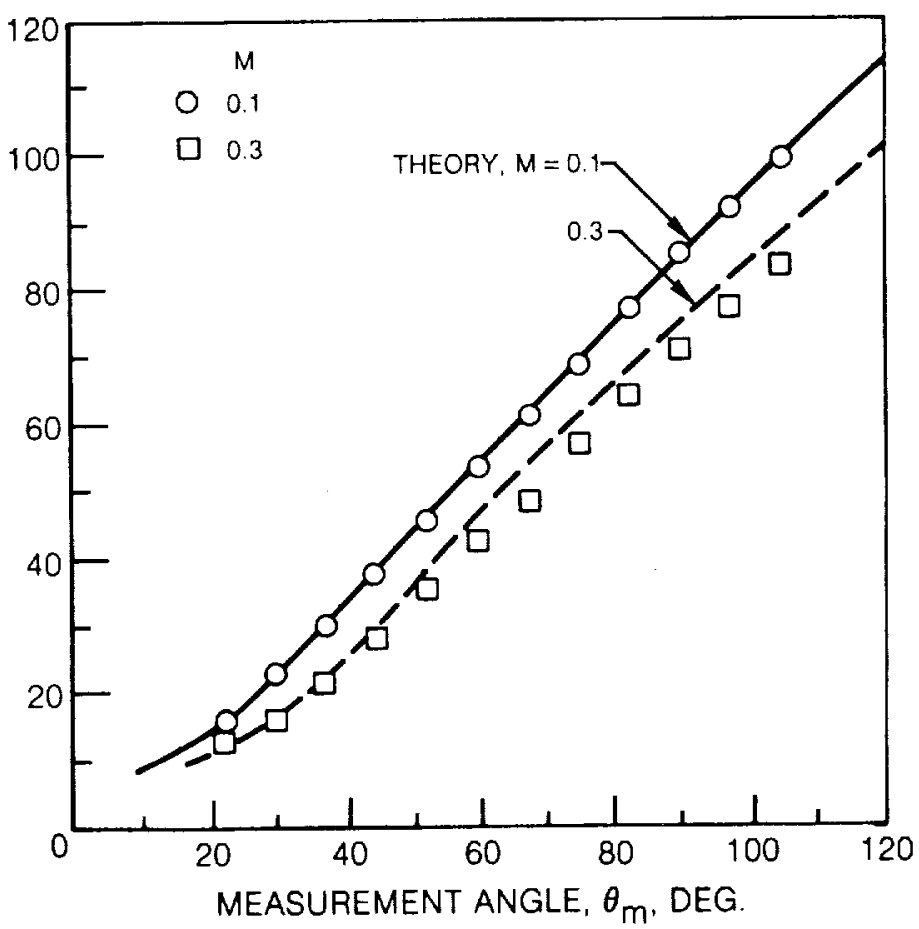

b) $f=10 \mathrm{kHz}$

Figure 42 - Comparison of Measured and Theoretical Refraction Angle Correction as a Function of Mach Number and Source Frequency, Test Configuration 4. 


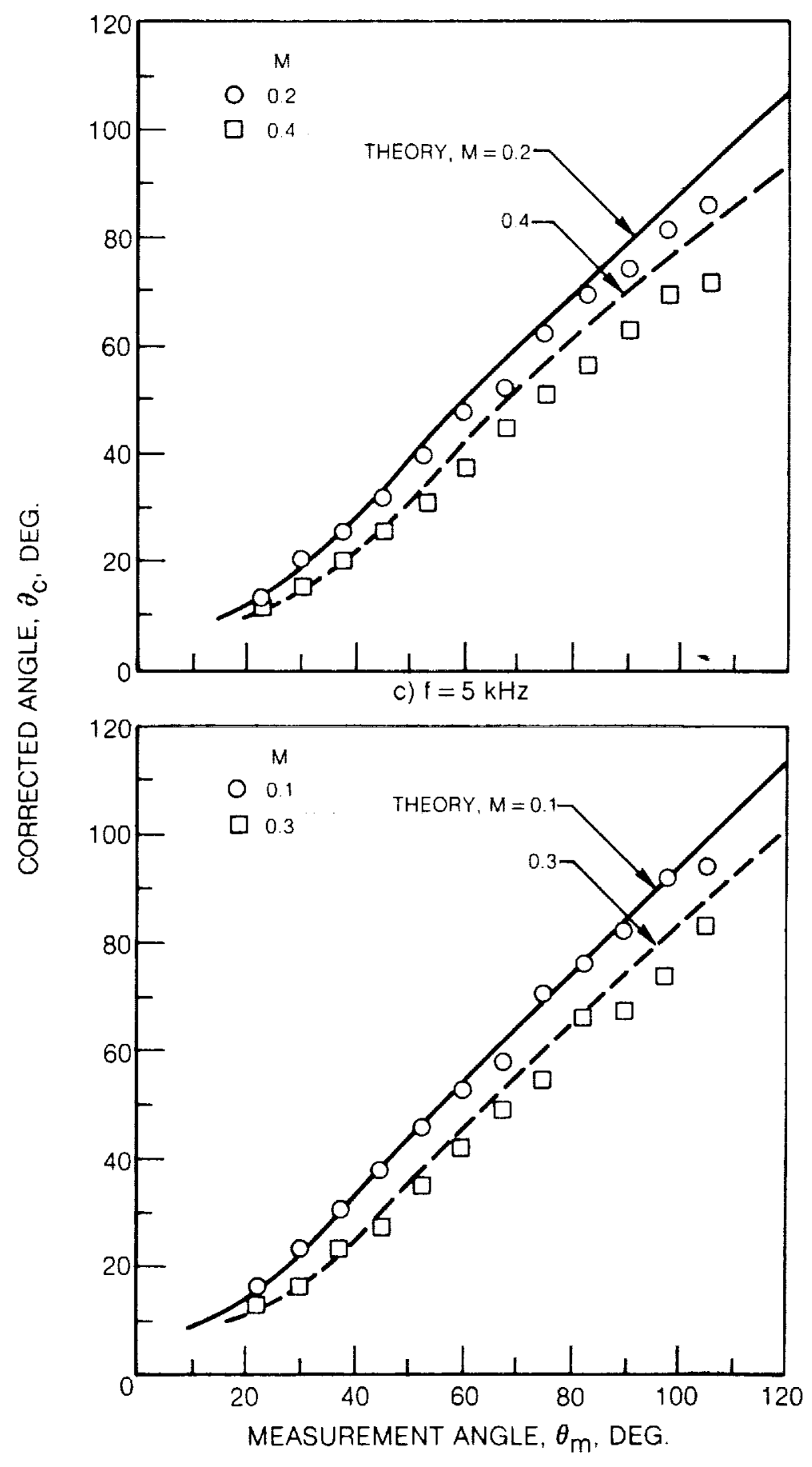

d) $f=5 \mathrm{kHz}$

Figure $42-$ Continued. 

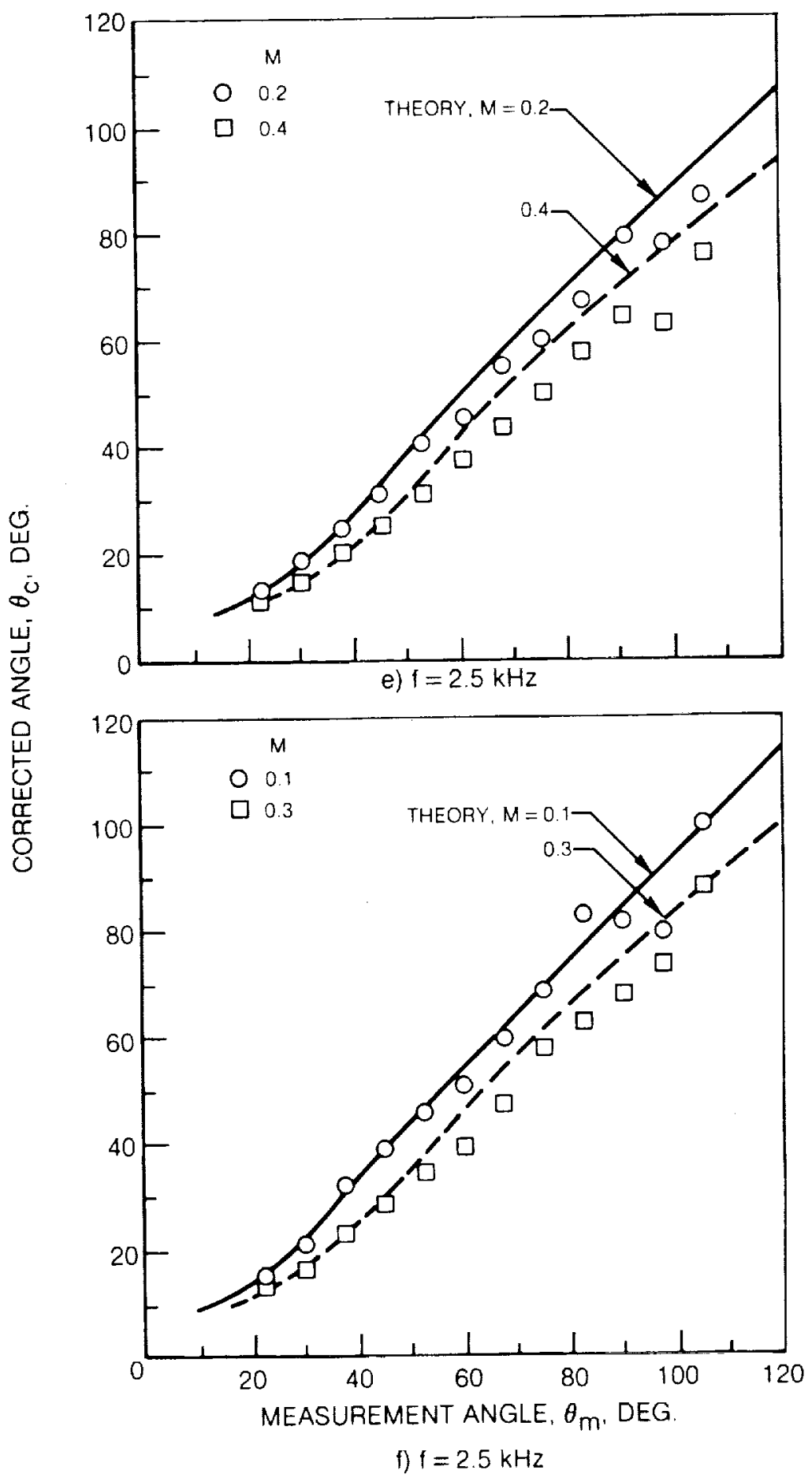

Figure 42 - Concluded. 

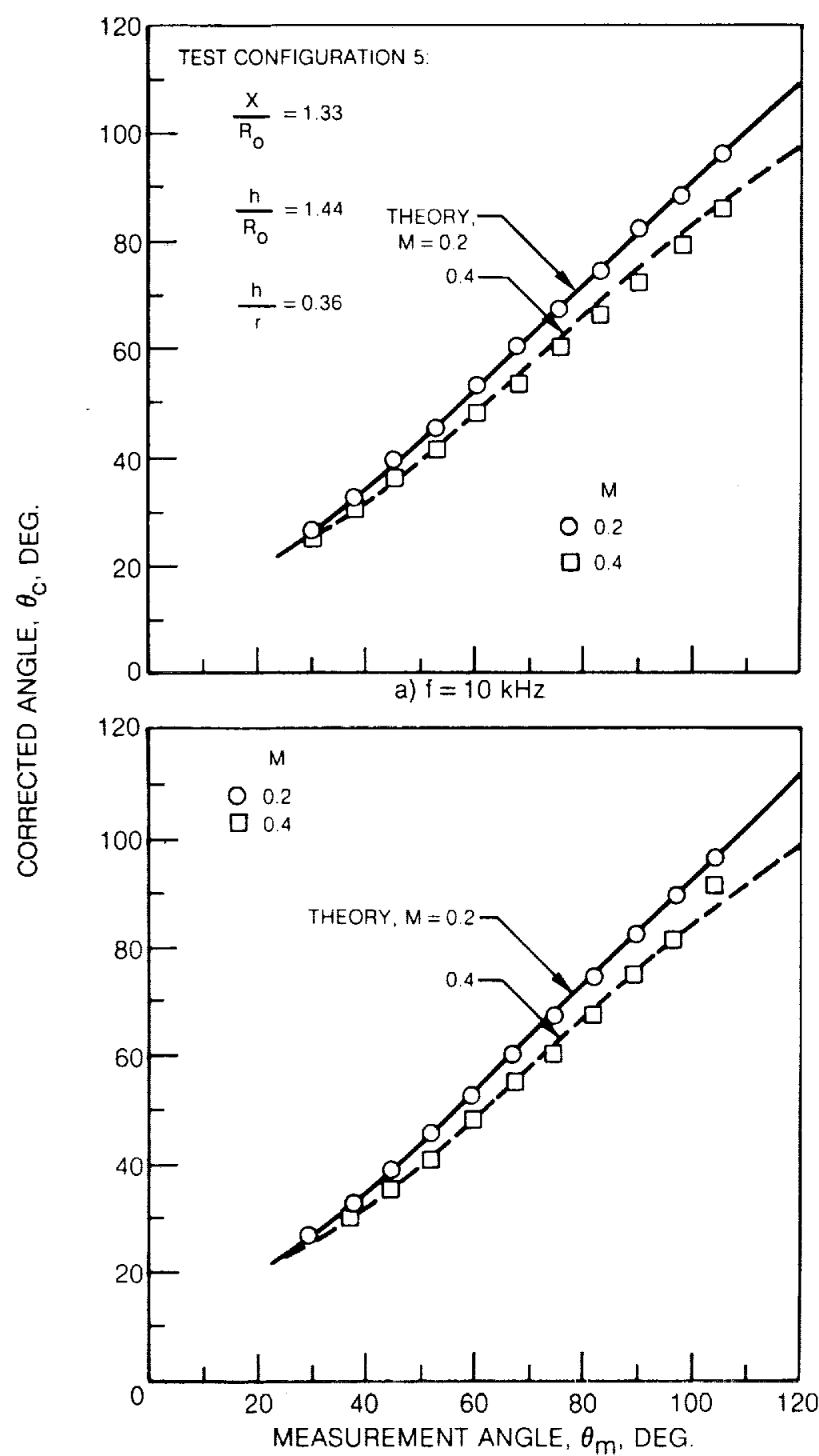

b) $f=5 \mathrm{kHz}$

Figure 43 - Comparison of Experimental and Theoretical Refraction Angle Correction as a Function of Mach Number and Frequency, Test Configuration 5. 


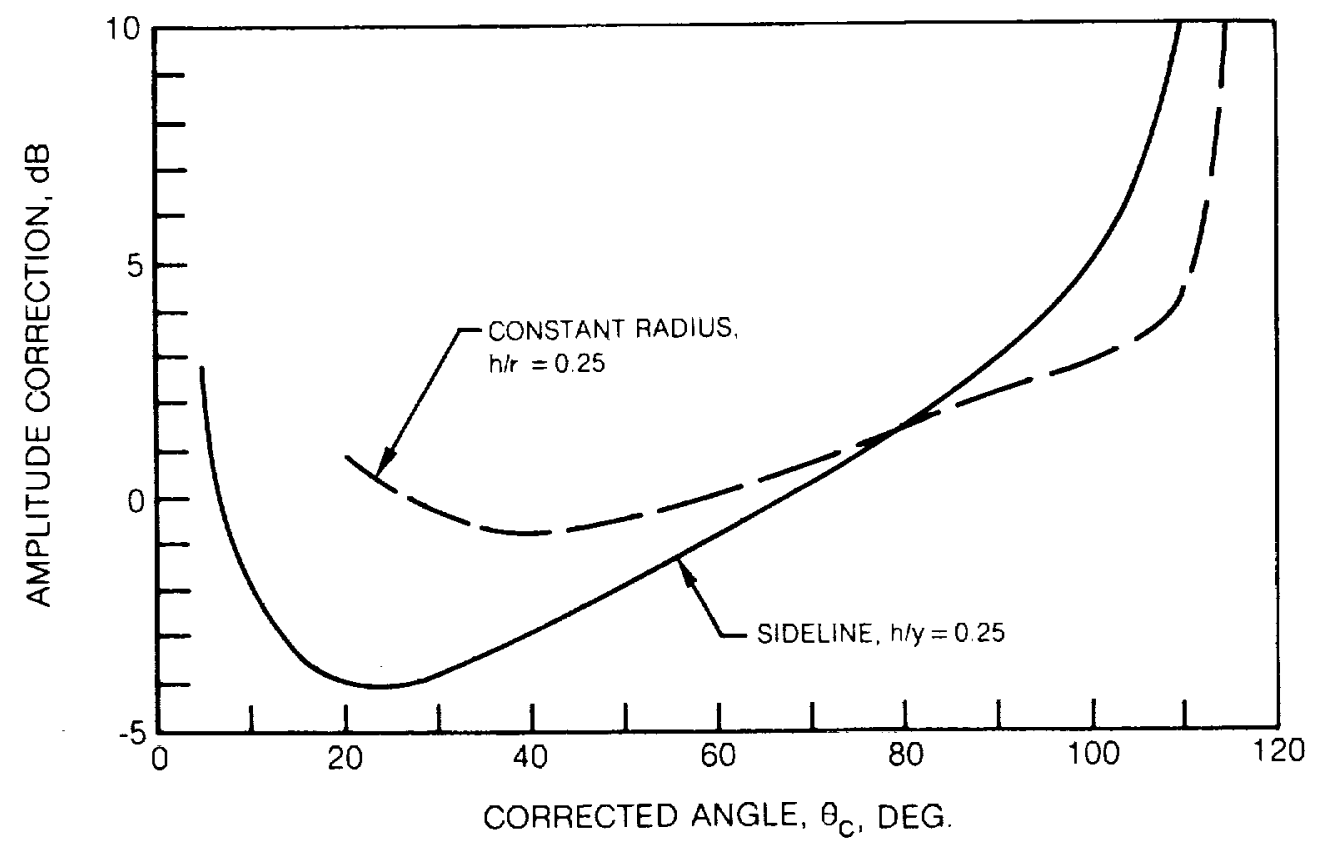

Figure 44 - Comparison of Theoretical Refraction Amplitude Correction for Sideline and Constant Radius Far Field Microphone Location with $M=0.4$, Source On-Axis. 

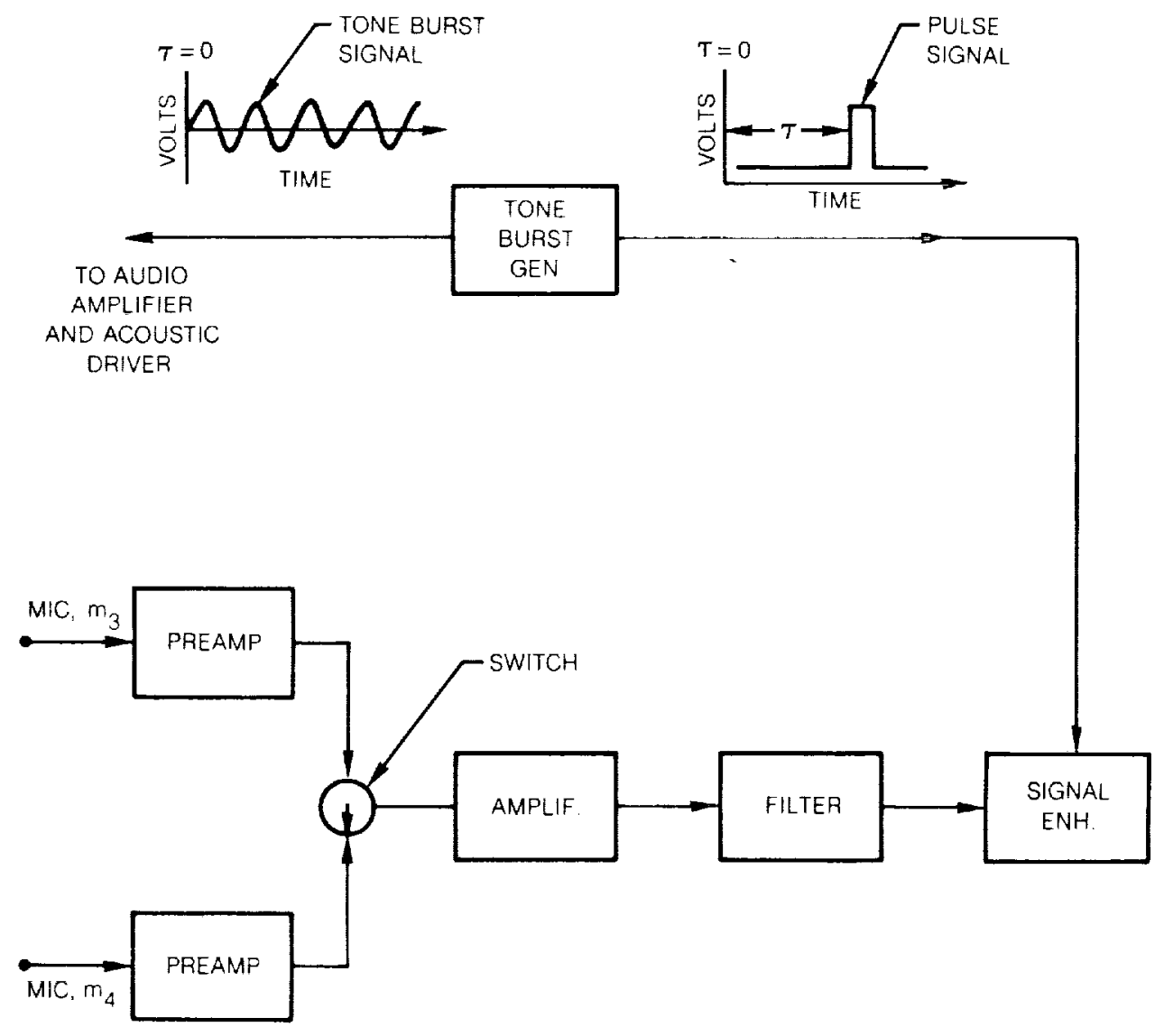

Figure 45a - Schematic of Signal Enhancement Circuitry Used to Capture Tone Burst. 


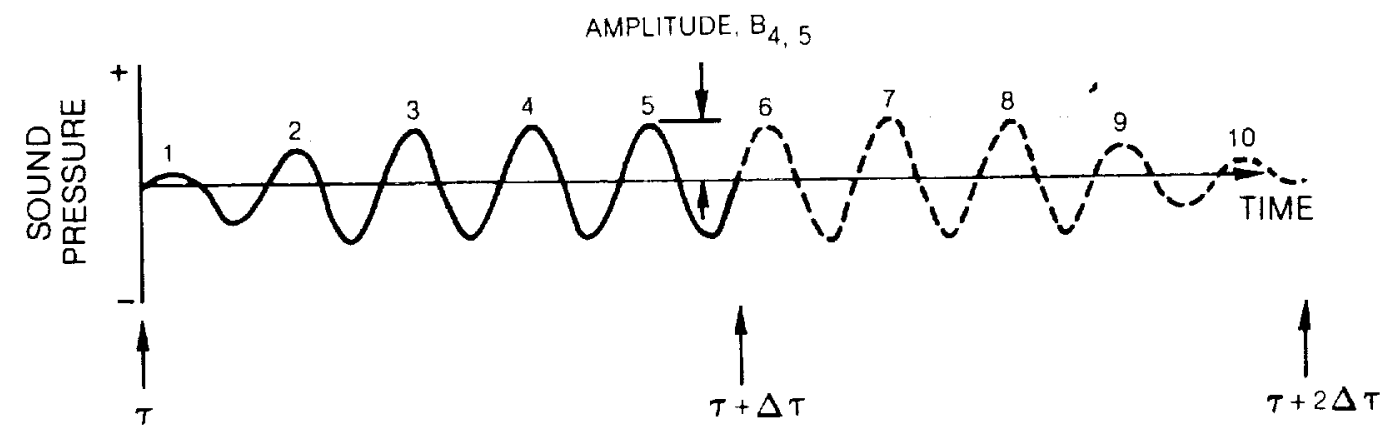

Figure 45b - Tone Burst Signal Recovered from Signal Enhancement. 


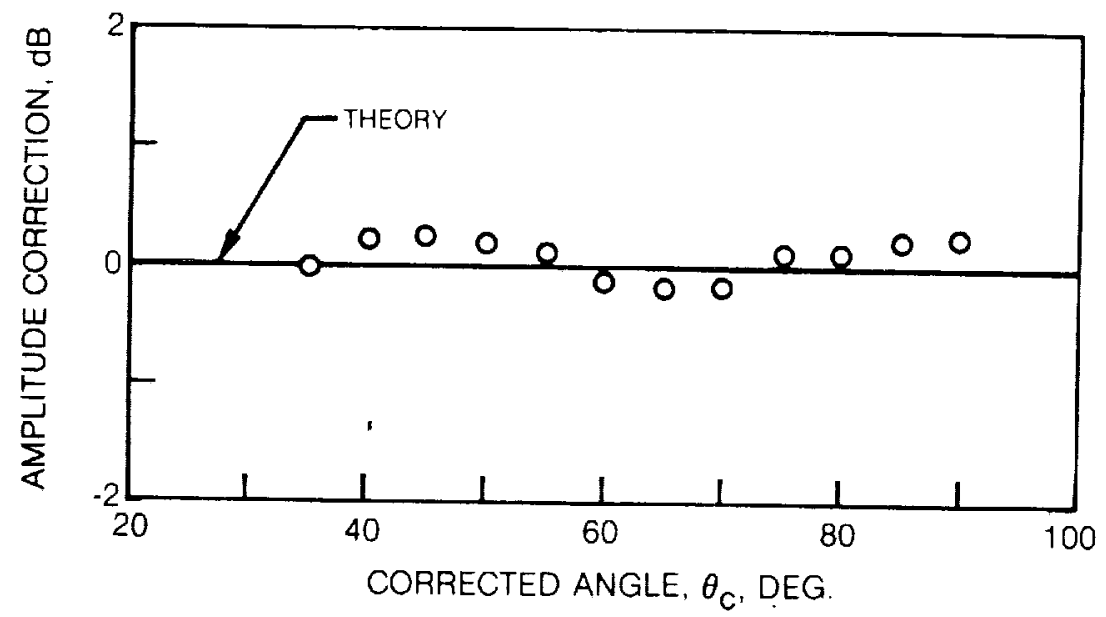

Figure 46 - Comparison of Measured and Theoretical Amplitude Correction, $M=0, f=5 \mathrm{kHz}$ Tone Burst, Test Configuration 6. 

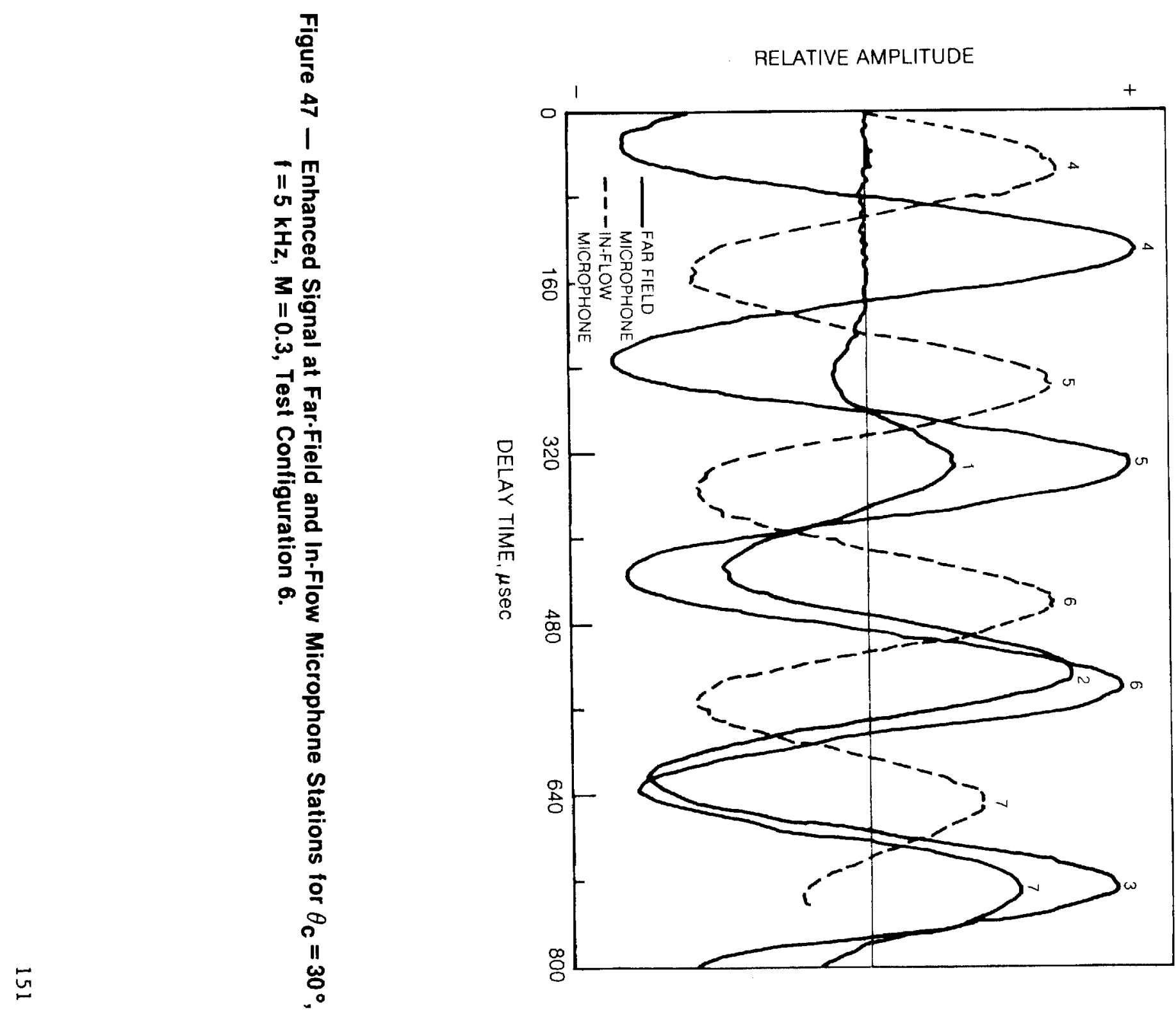


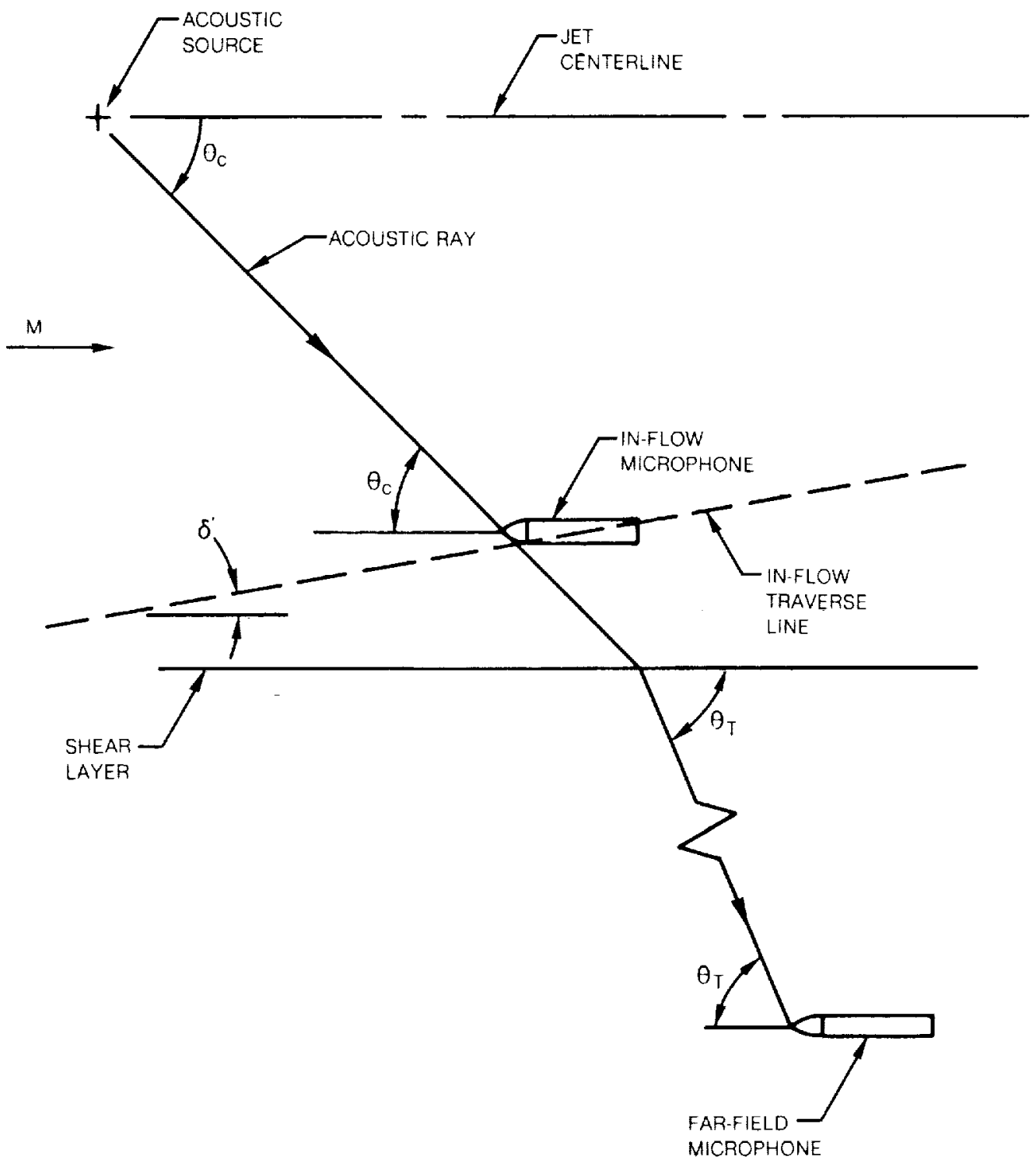

Figure 48 - Acoustic Ray Orientation Relative to Microphones. 


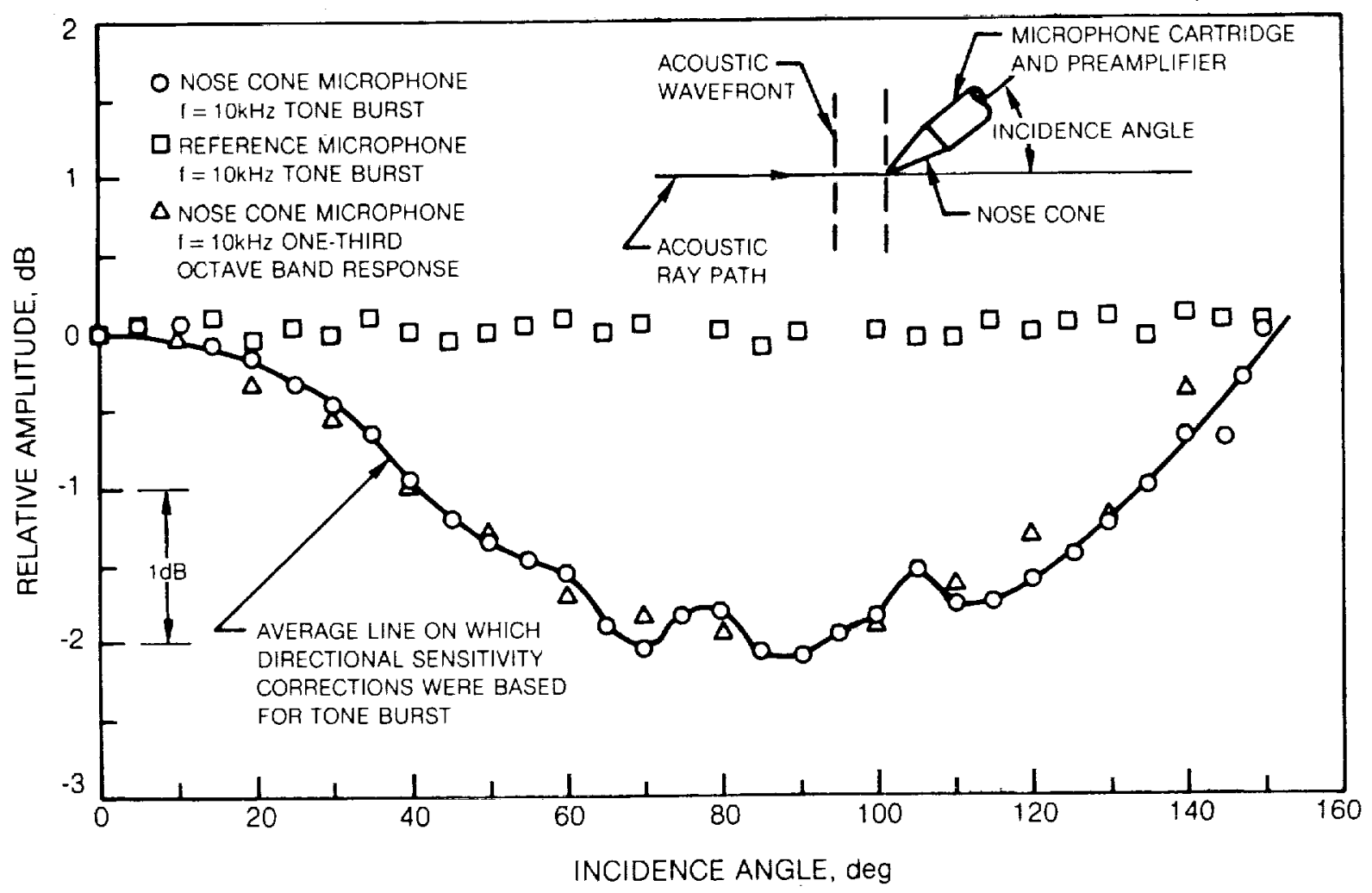

Figure 49 - Nose Cone Directional Sensitivity to Tone Burst and Random Noise Signals. 
'pepn|ou०o - 6b әมn6!]

6әр ' $\exists า ด N \forall ~ \exists O N \exists \triangle I O N I$

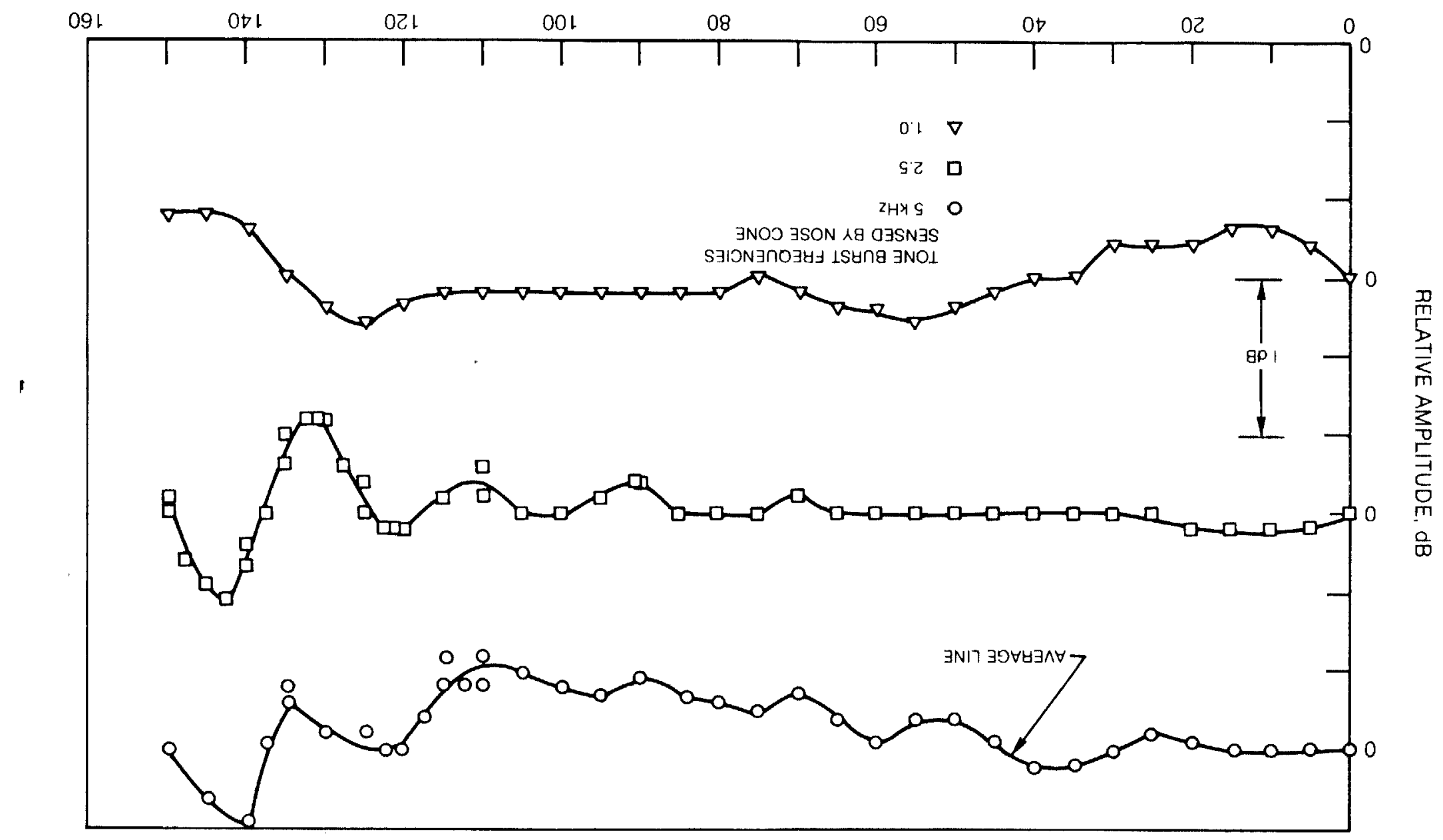




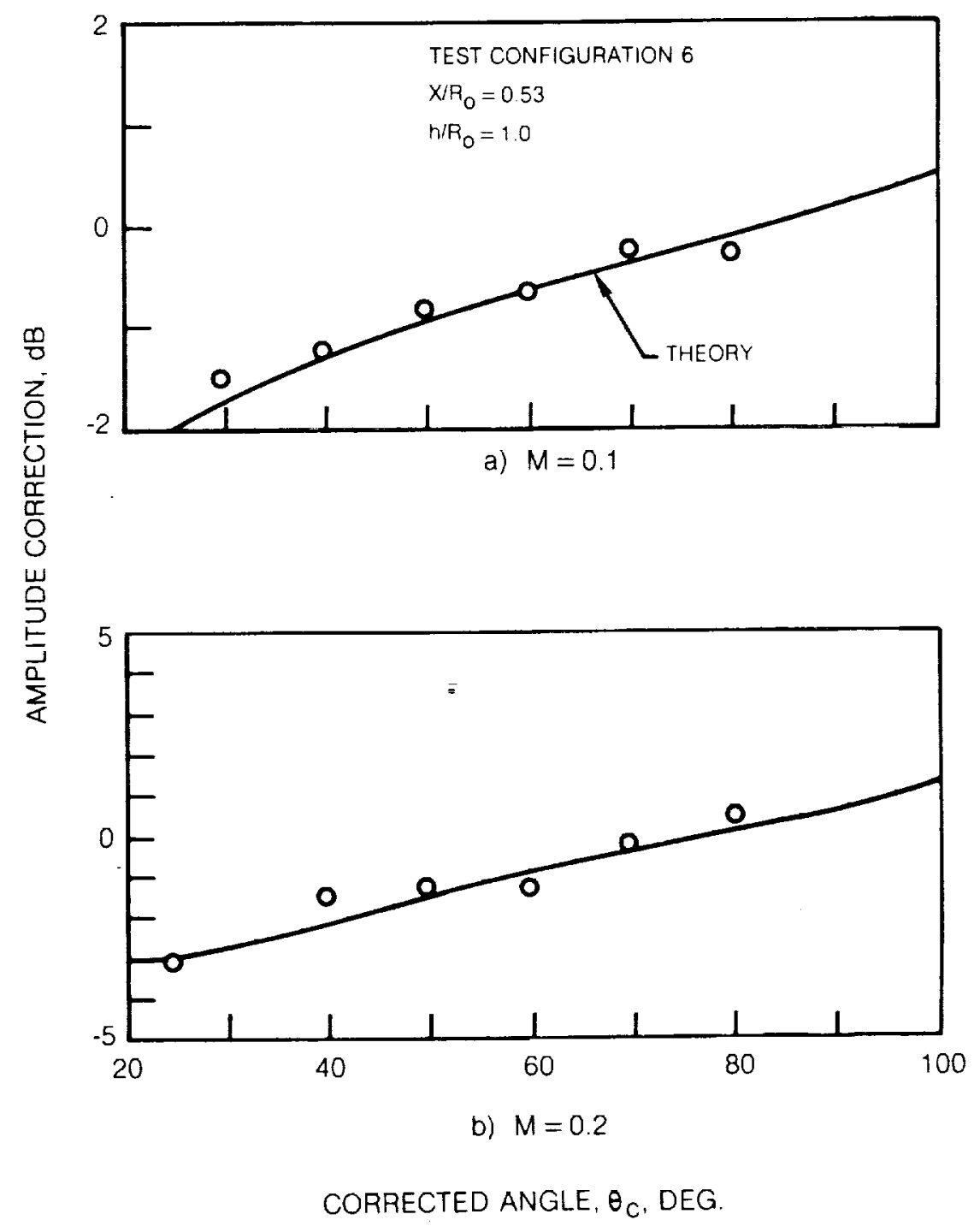

Figure 50 - Comparison of Measured and Theoretical Refraction Amplitude Correction as a Function of Mach Number for $f=1.25 \mathrm{kHz}$, Test Configuration 6. 


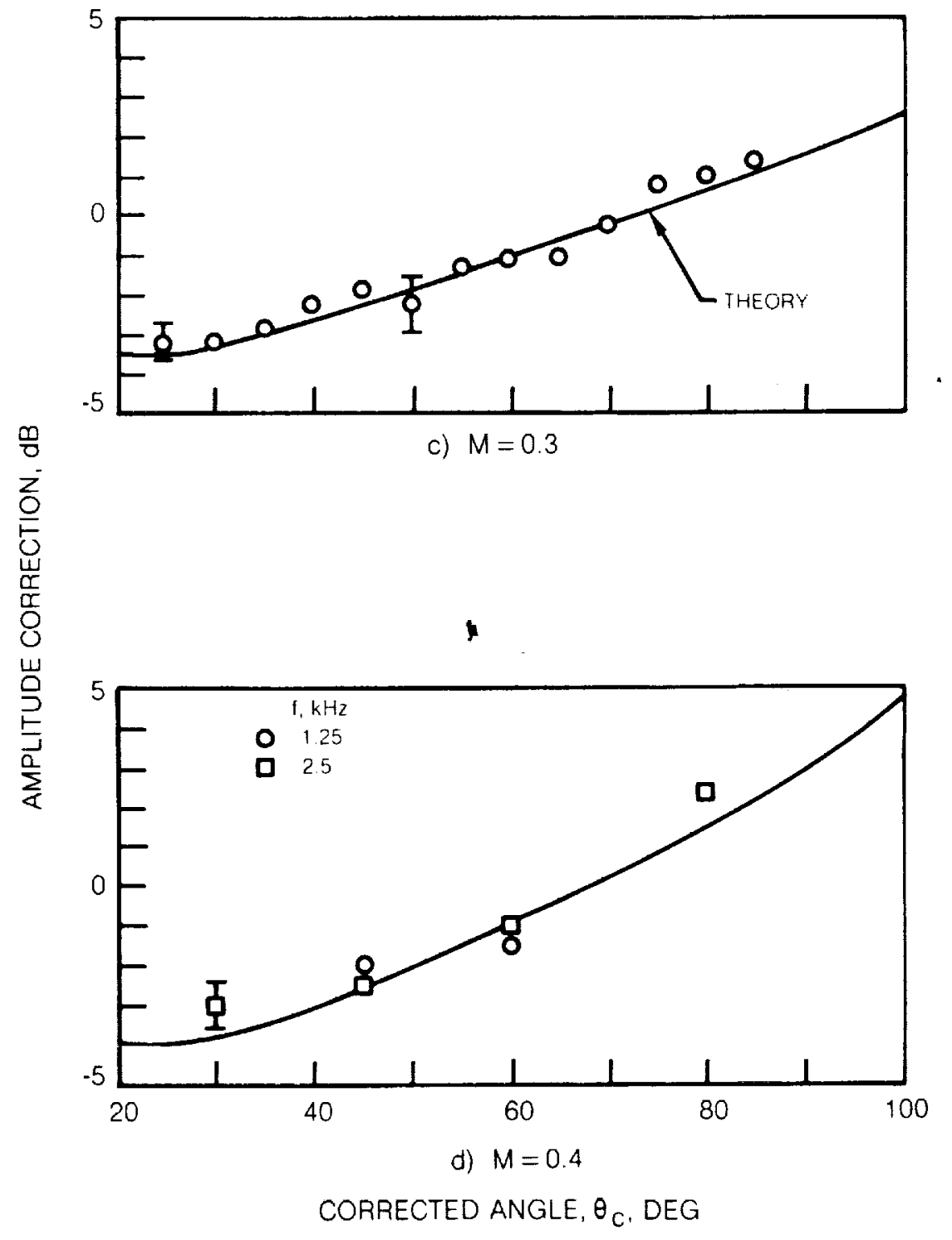

Figure 50 - Concluded. 


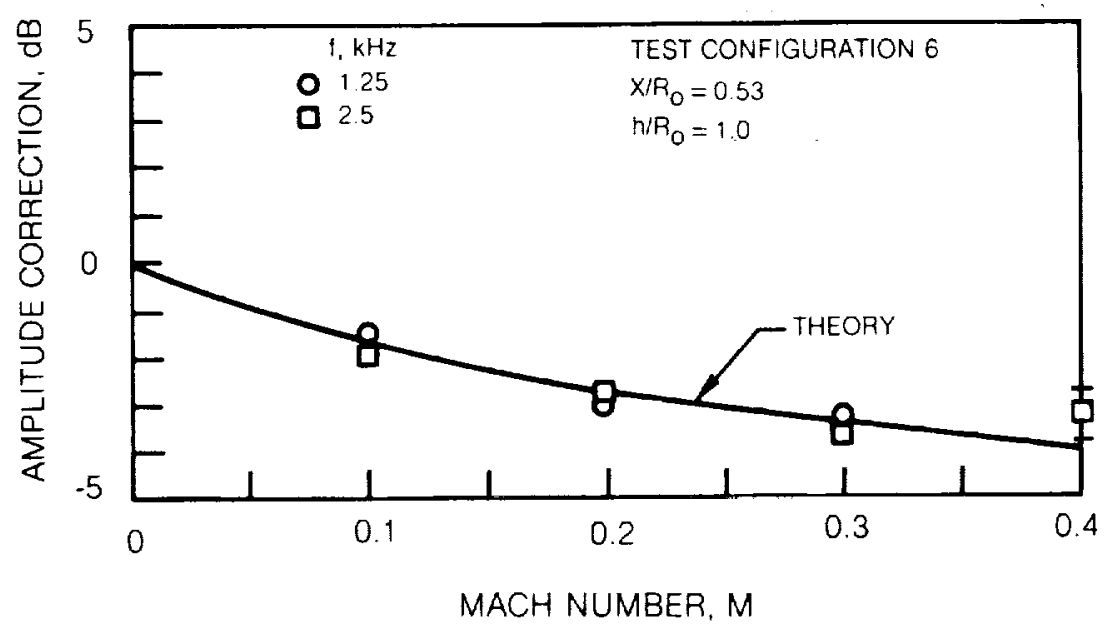

Figure 51 - Comparison of Measured and Theoretical Refraction Amplitude Correction as a Function of Mach Number for $\theta_{c}=30^{\circ}$, Test Configuration 6 . 


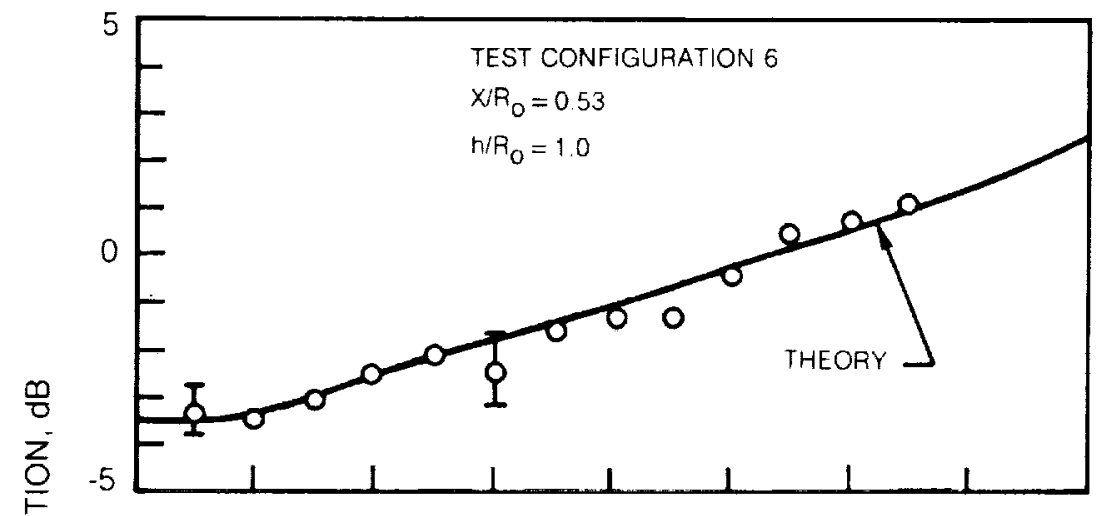

a) $f=1.25 \mathrm{kHz}$

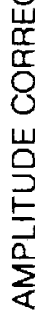

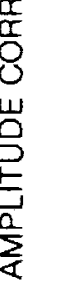

5

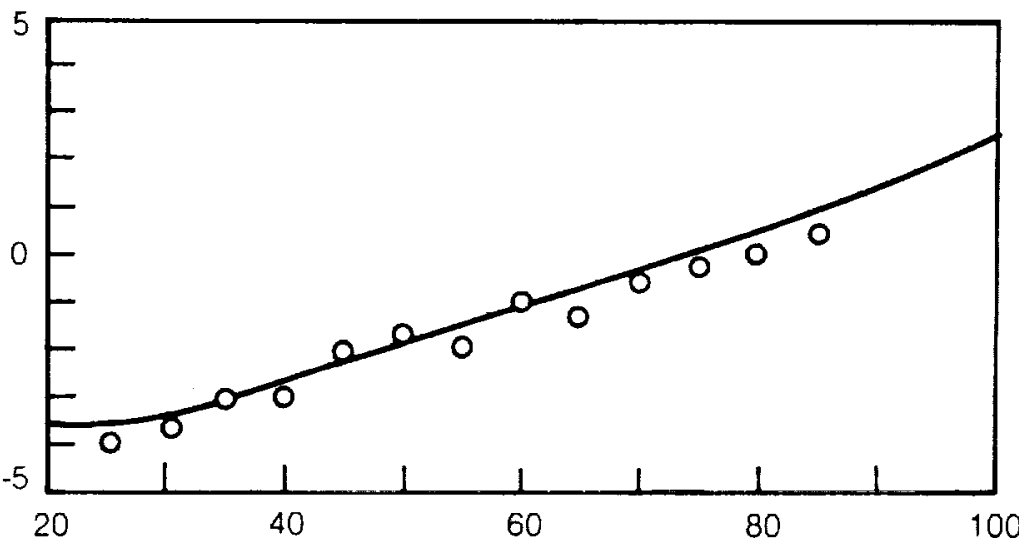

b) $\mathrm{I}=2.5 \mathrm{kHz}$

CORRECTED ANGLE, $\theta_{\mathrm{C}}$, DEG

Figure 52 - Comparison of Measured and Theoretical Refraction Amplitude Correction as a Function of Frequency for $M=0.3$

Test Configuration 6 . 


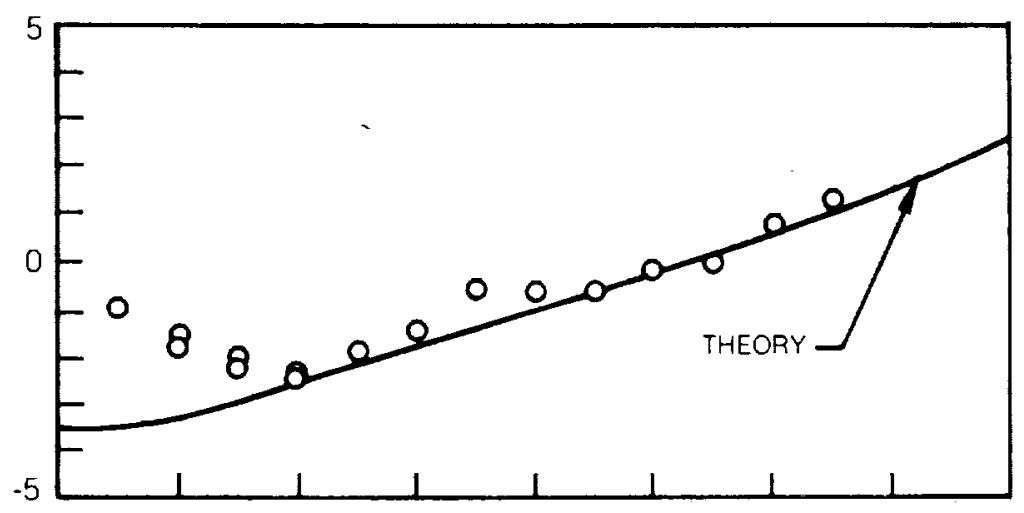

c) $f=5 \mathrm{kHz}$

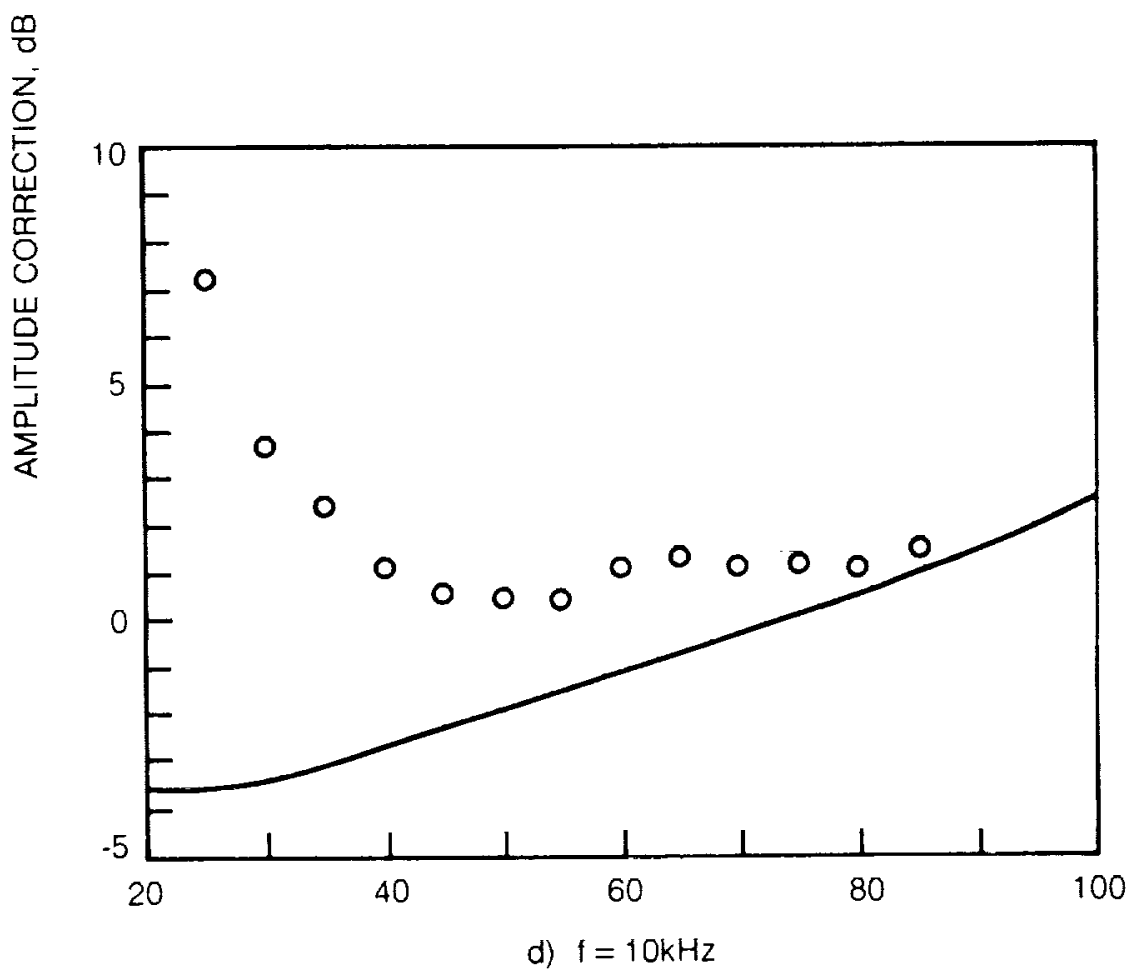

CORRECTED ANGLE, $\theta_{\mathrm{C}}, \mathrm{DEG}$

Figure 52 - Concluded. 


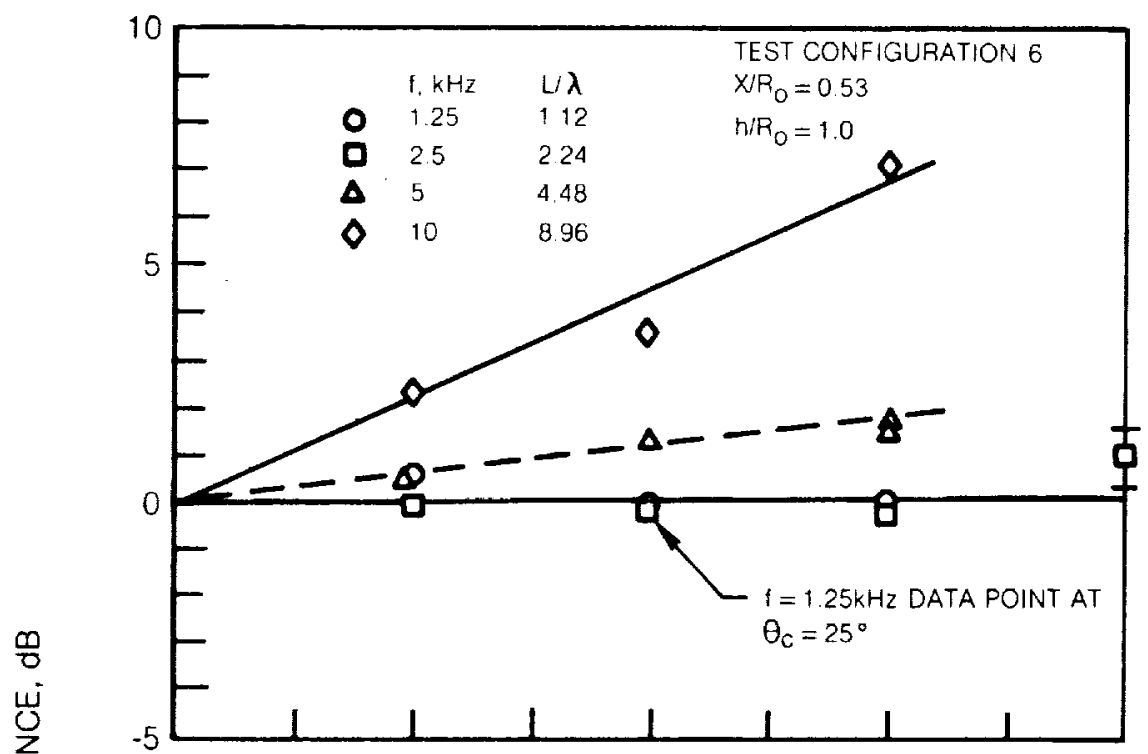

a) $\theta_{C}=30^{\circ}$

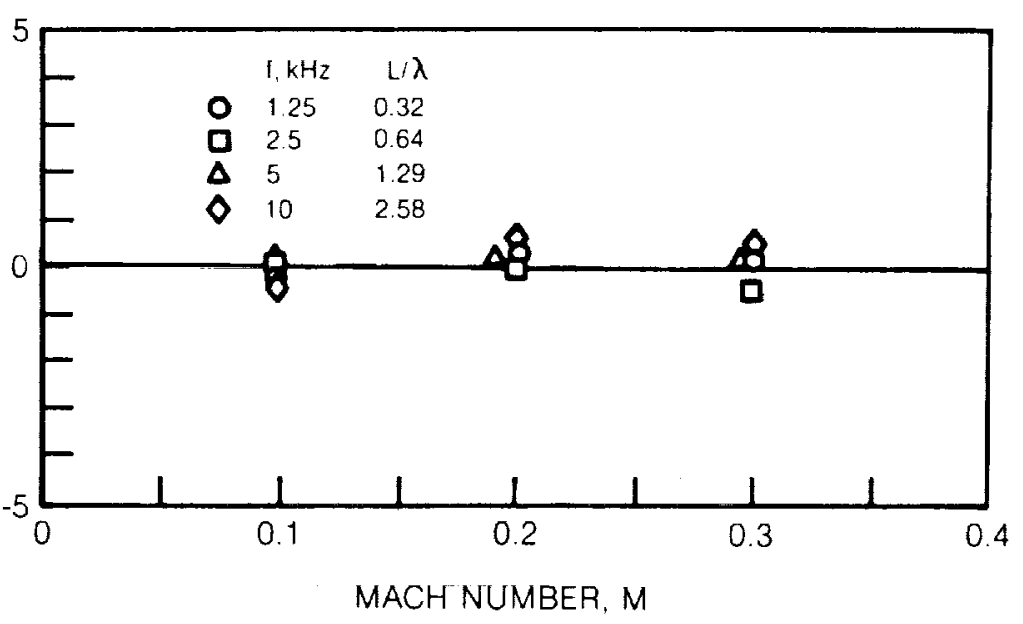

b) $\theta_{C}=80^{\circ}$

Figure 53 - Difference Between Measured and Theoretical Refraction Amplitude Correction as a Function of Frequency, Mach Number, and Corrected Angle, Test Configuration 6 . 


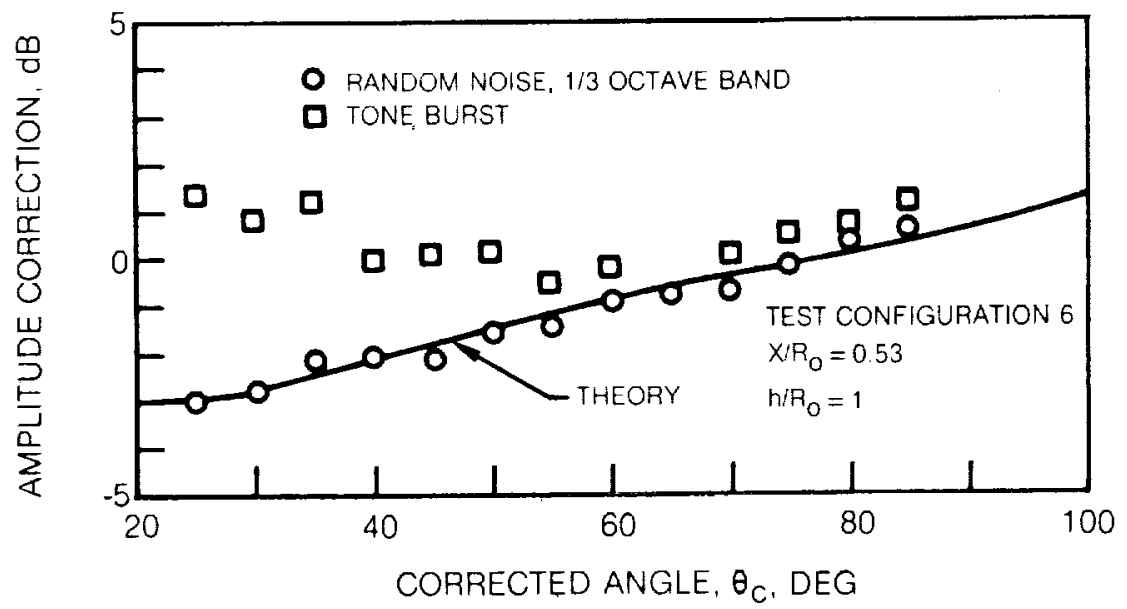

Figure 54 - Comparison of Measured and Theoretical Refraction Amplitude Correction for Tone Burst Noise Source and Random Noise Source, $f=10 \mathrm{kHz}, M=0.2$, Test Configuration 6 . 

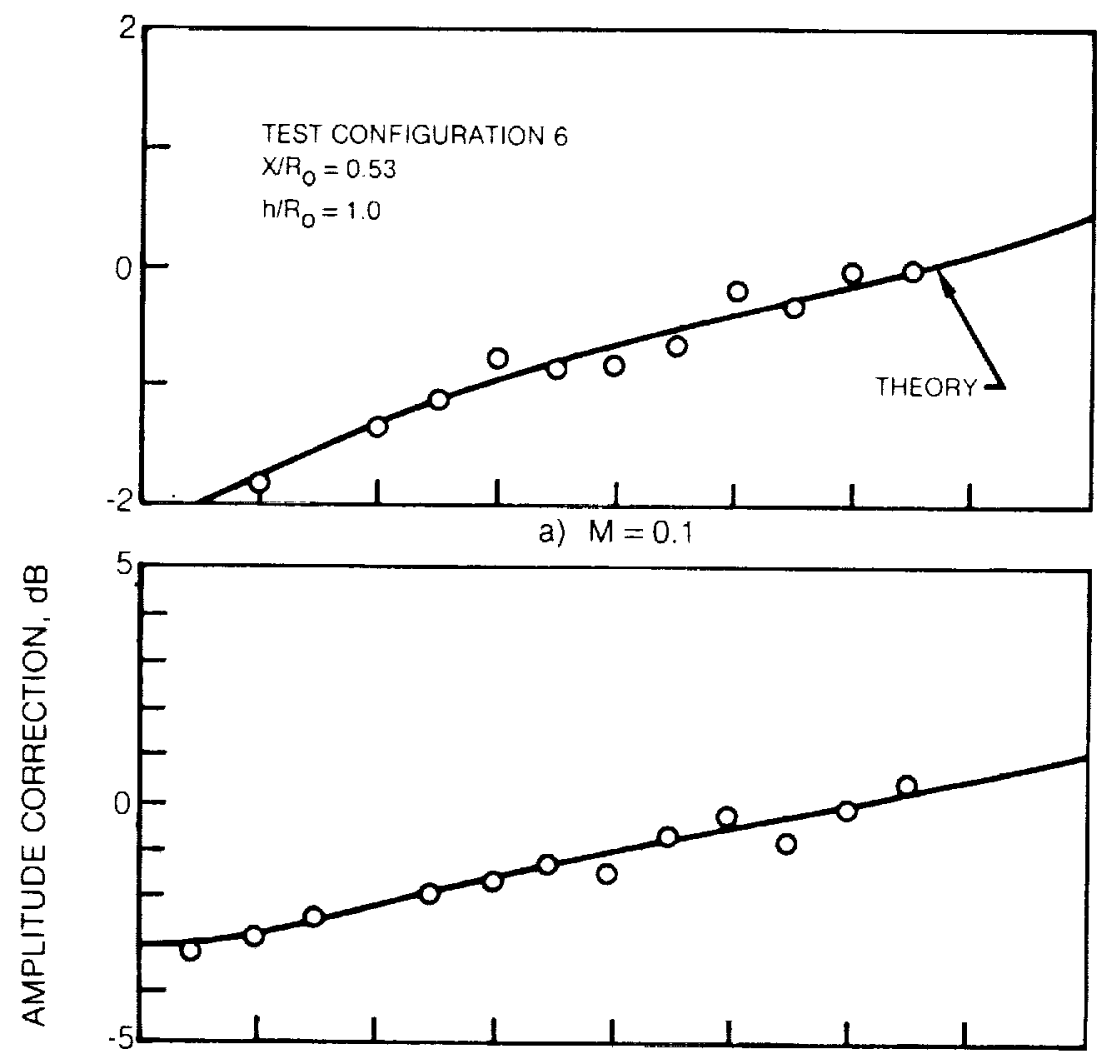

b) $M=0.2$

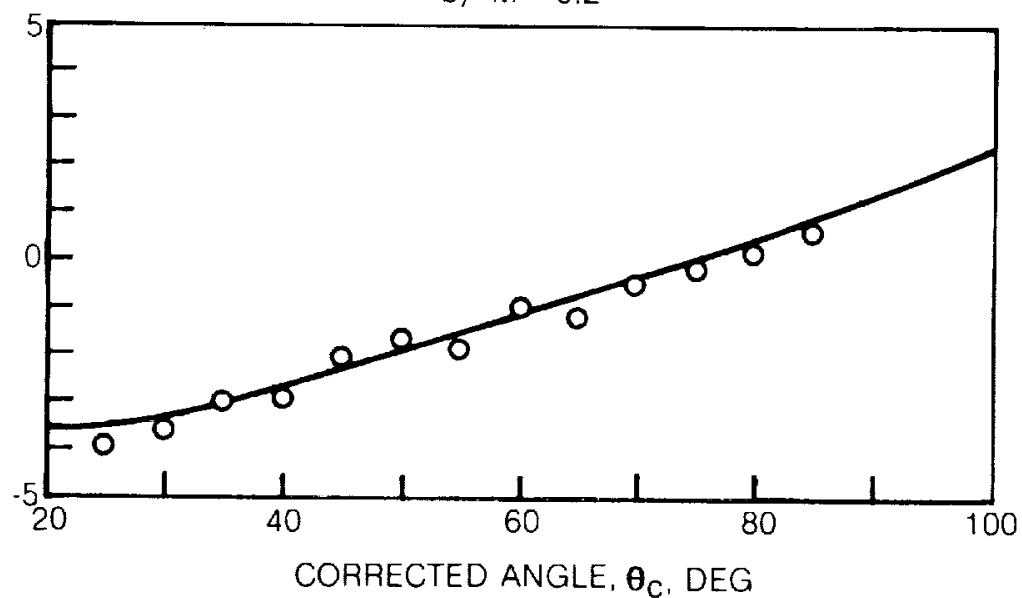

c) $M=0.3$

Figure 55 - Comparison of Measured and Theoretical Amplitude Correction as a Function of Mach Number for $f=2.25 \mathrm{kHz}$, Test Configuration 6 . 


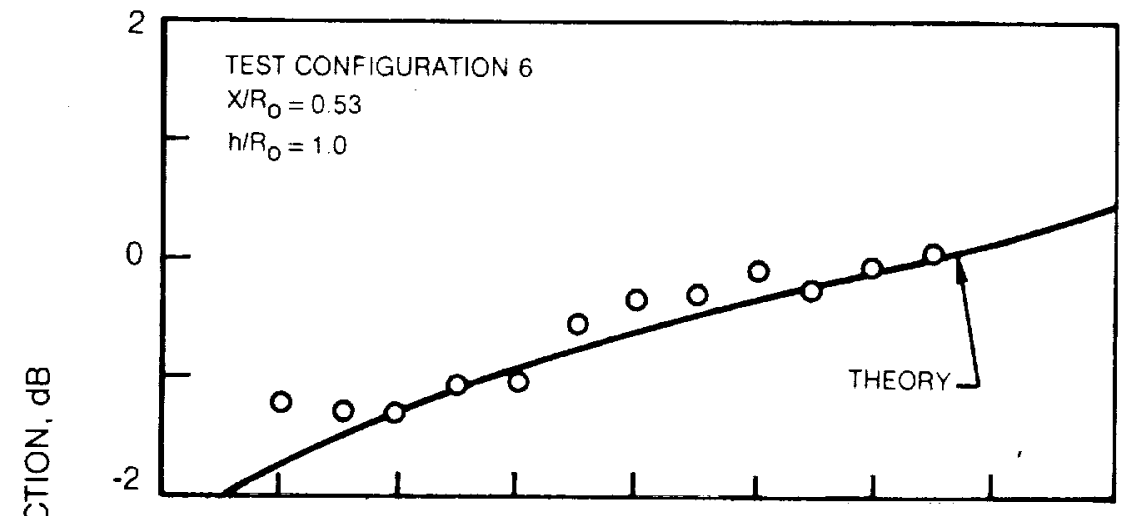

a) $M=0.1$

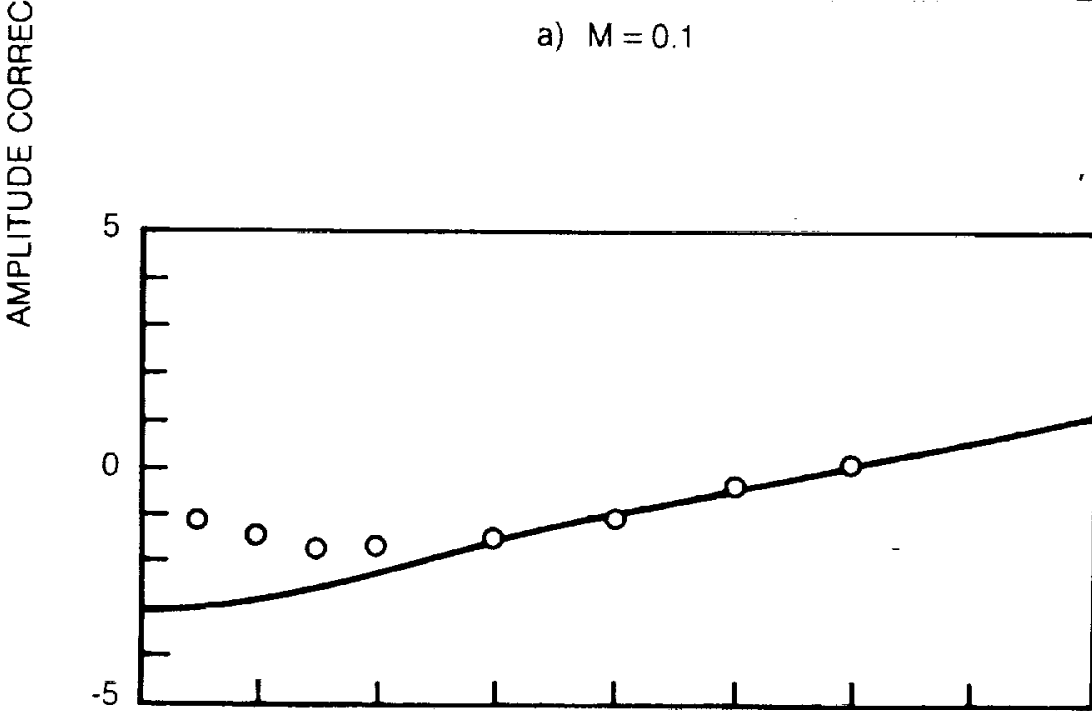

CORRECTED ANGLE, $\theta_{C}$, DEG

b) $M=0.2$

Figure 56 - Comparison of Measured and Theoretical Amplitde Correction as a Function of Mach Number for $f=5 \mathrm{kHz}$, Test Configuration 6 . 


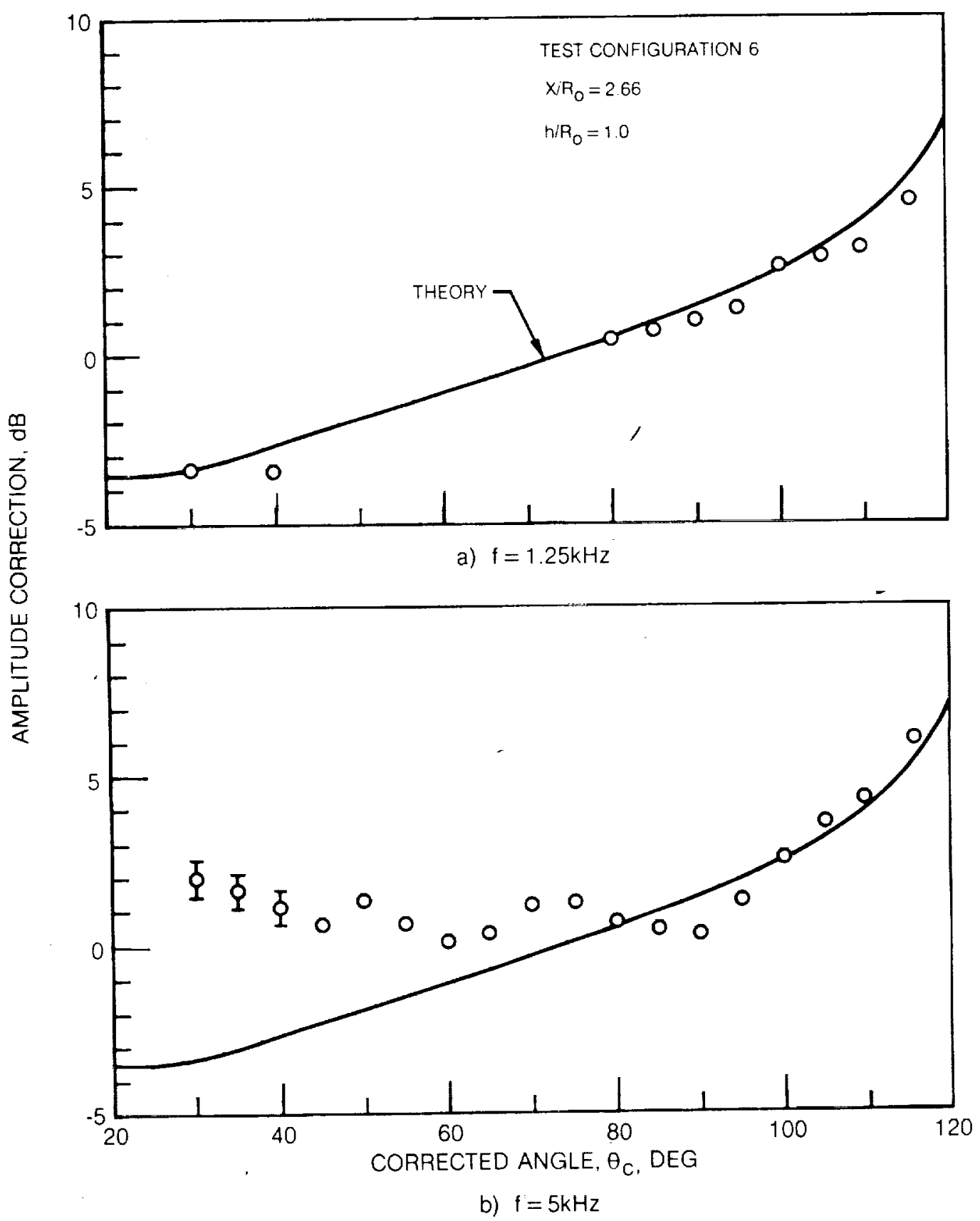

Figure 57 - Comparison of Measured and Theoretical Refraction Amplitude Correction as a Function of Frequency for $M=0.3$,

Test Configuration 7. 


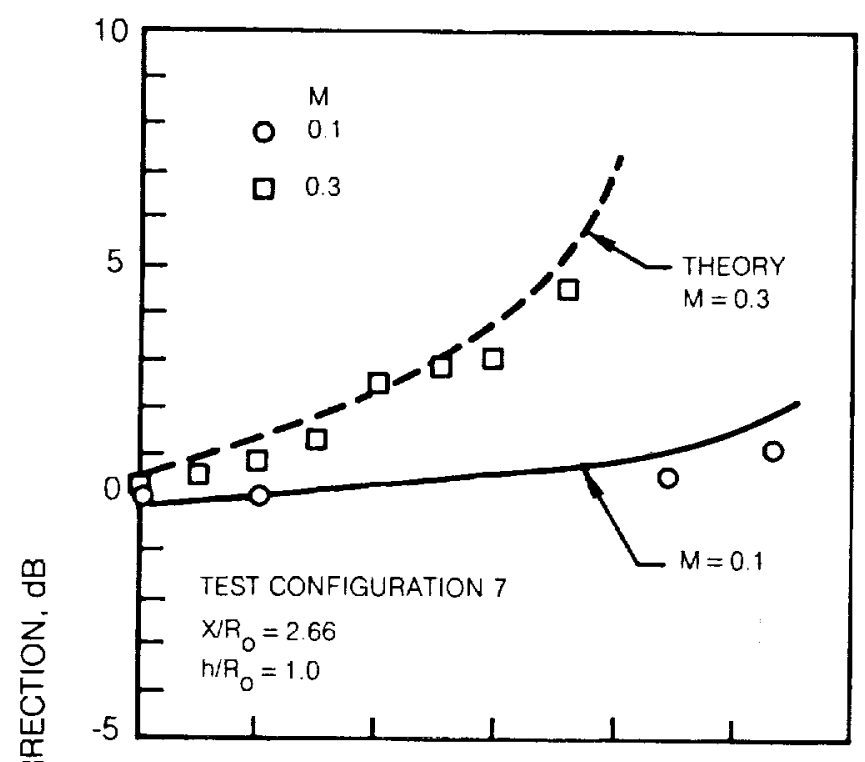

a) $M=0.1,0.3$

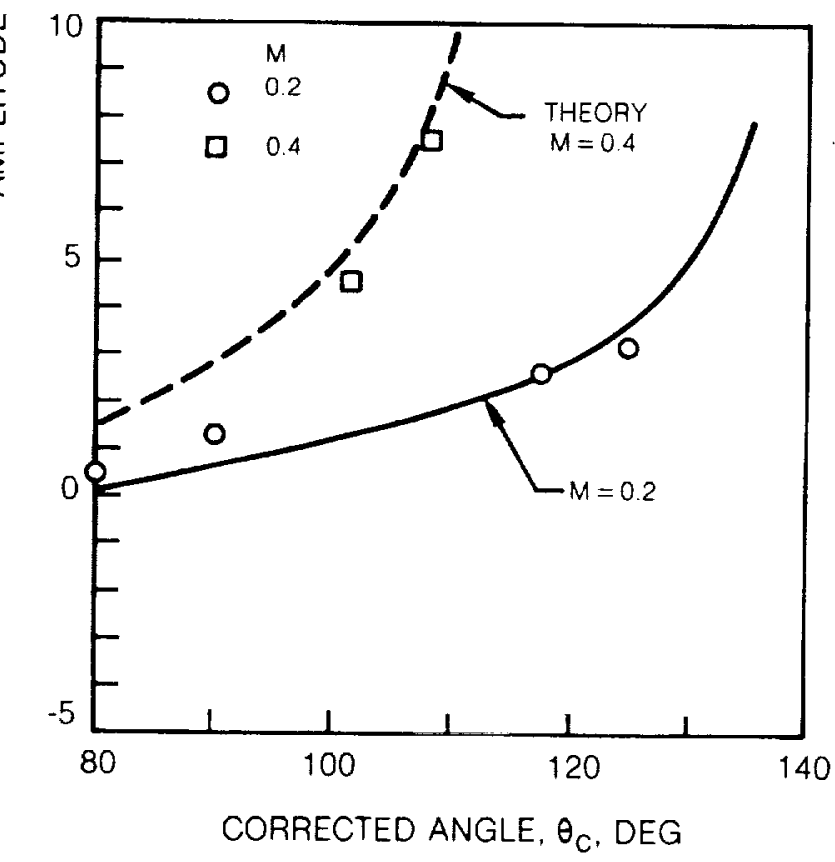

b) $M=0.2,0.4$

Figure 58 - Comparison of Measured and Theorectical Refraction Amplitude Correction in the Forward Quadrant for Varying Mach Number with $i=1.25 \mathrm{kHz}$, Test Configuration 7 . 


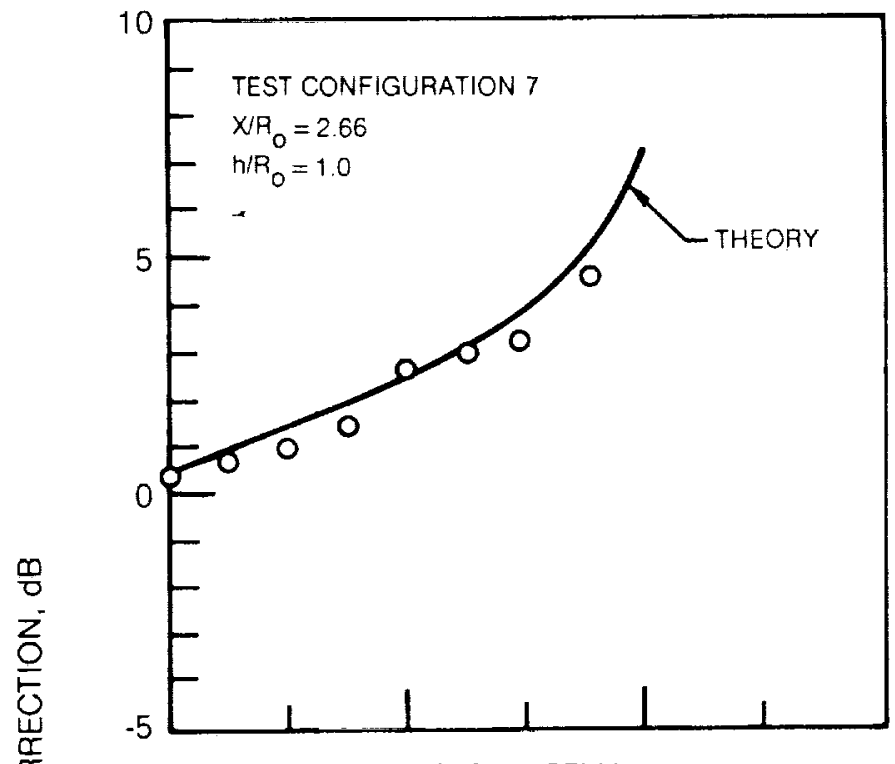

a) $f=1.25 \mathrm{kHz}$

능

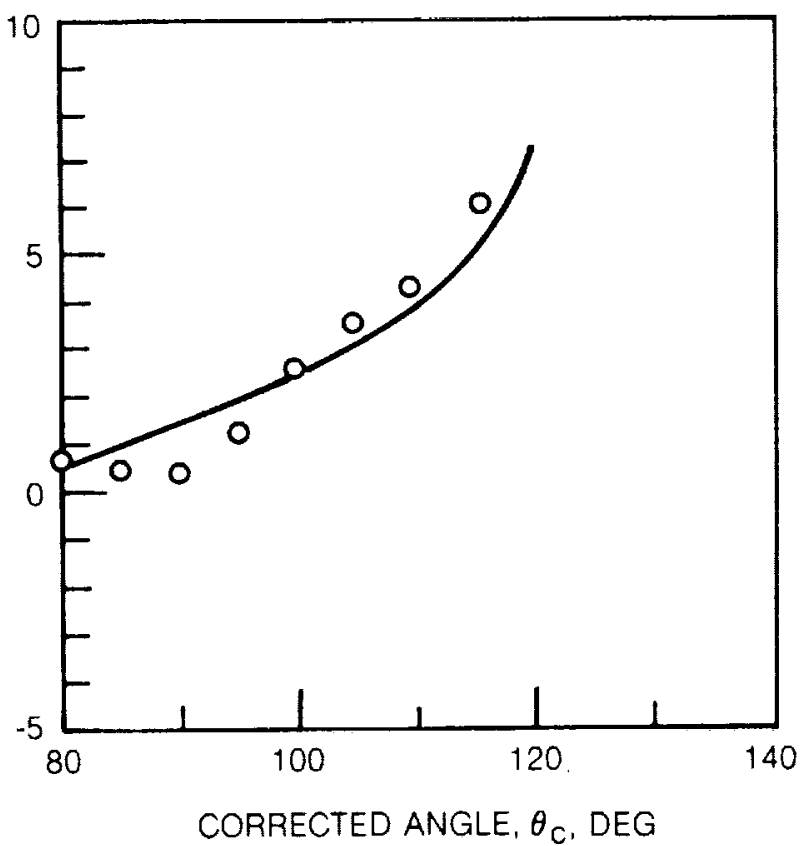

b) $f=5 \mathrm{kHz}$

Figure 59 - Comparison of Measured and Theoretical Refraction Amplitude Correction in the Forward Quadrant for Varying Source Frequency with $M=0.3$, Test Configuration 7 . 
AMPLITUDE CORRECTION, dB

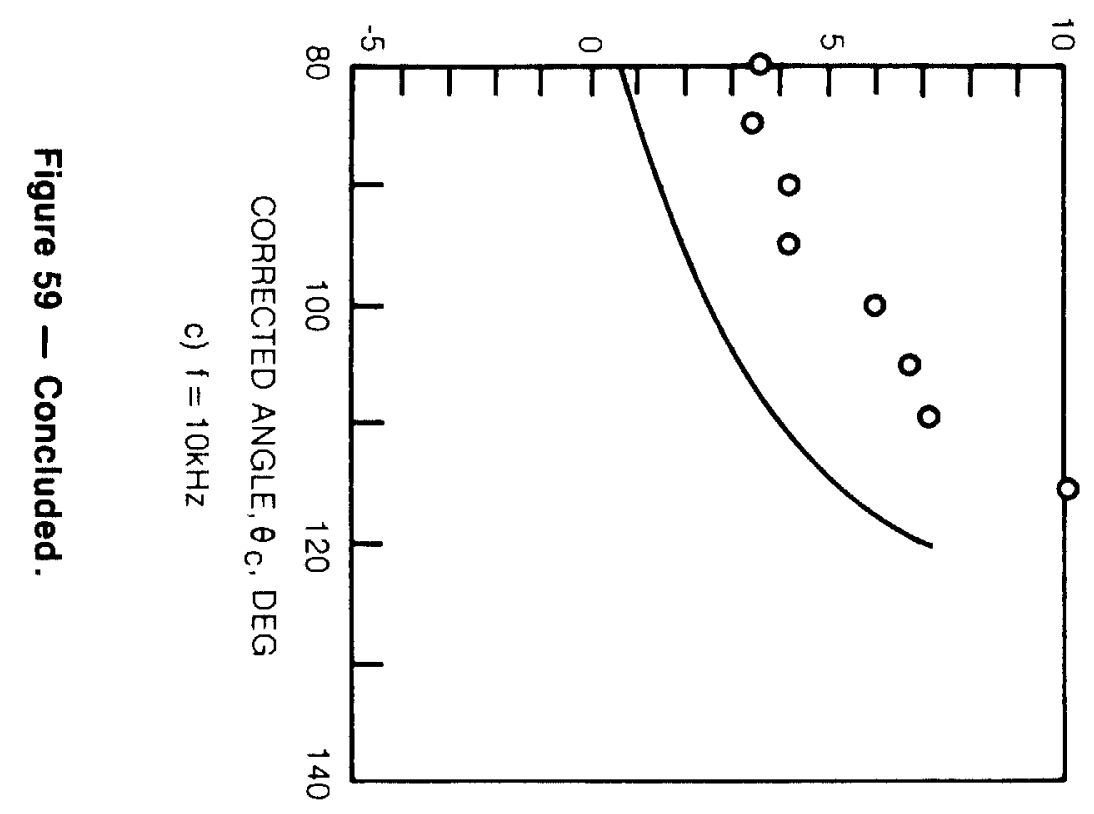




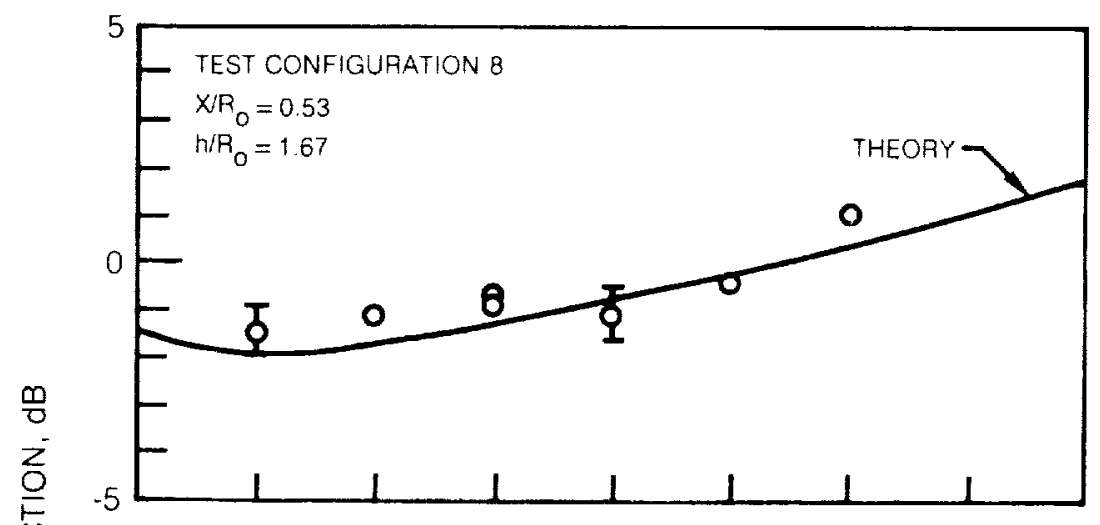

a) $f=1.25 \mathrm{kHz}$

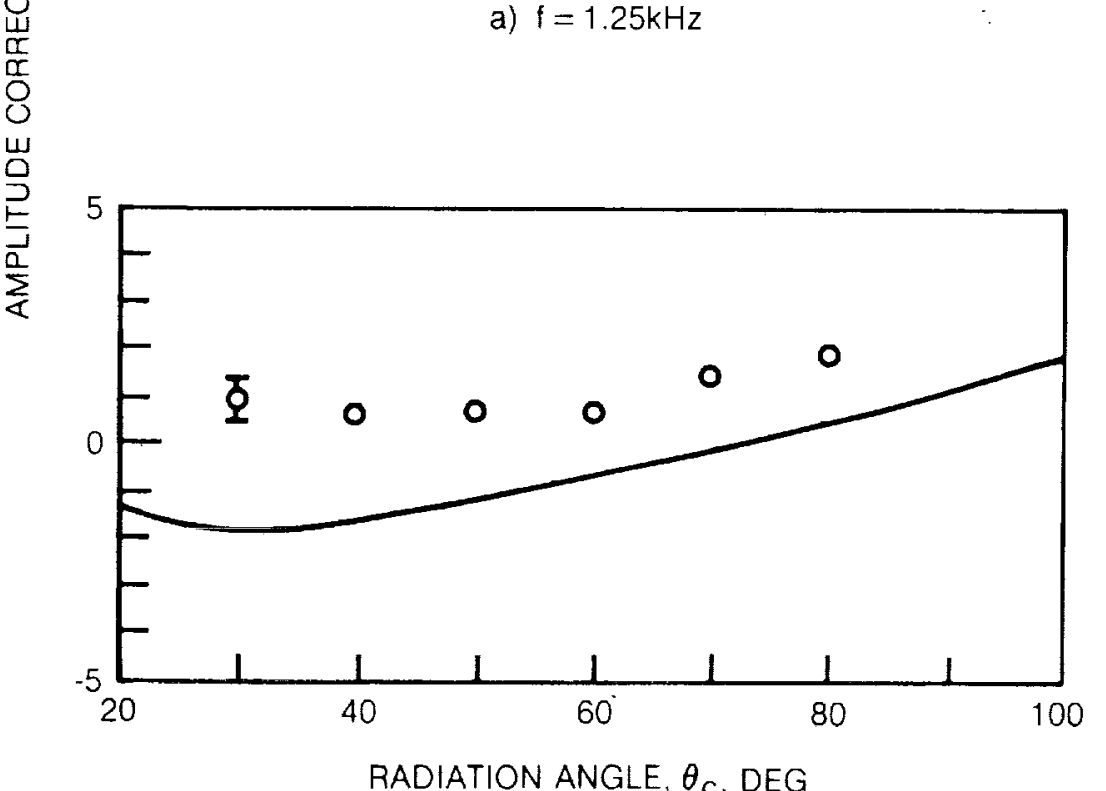

RADIATION ANGLE, $\theta_{C}$, DEG

b) $f=5 \mathrm{kHz}$

Figure 60 - Comparison of Measured and Theoretical Refraction Amplitude Correction as a Function of Frequency for $M=0.3$, Test Configuration 8 . 


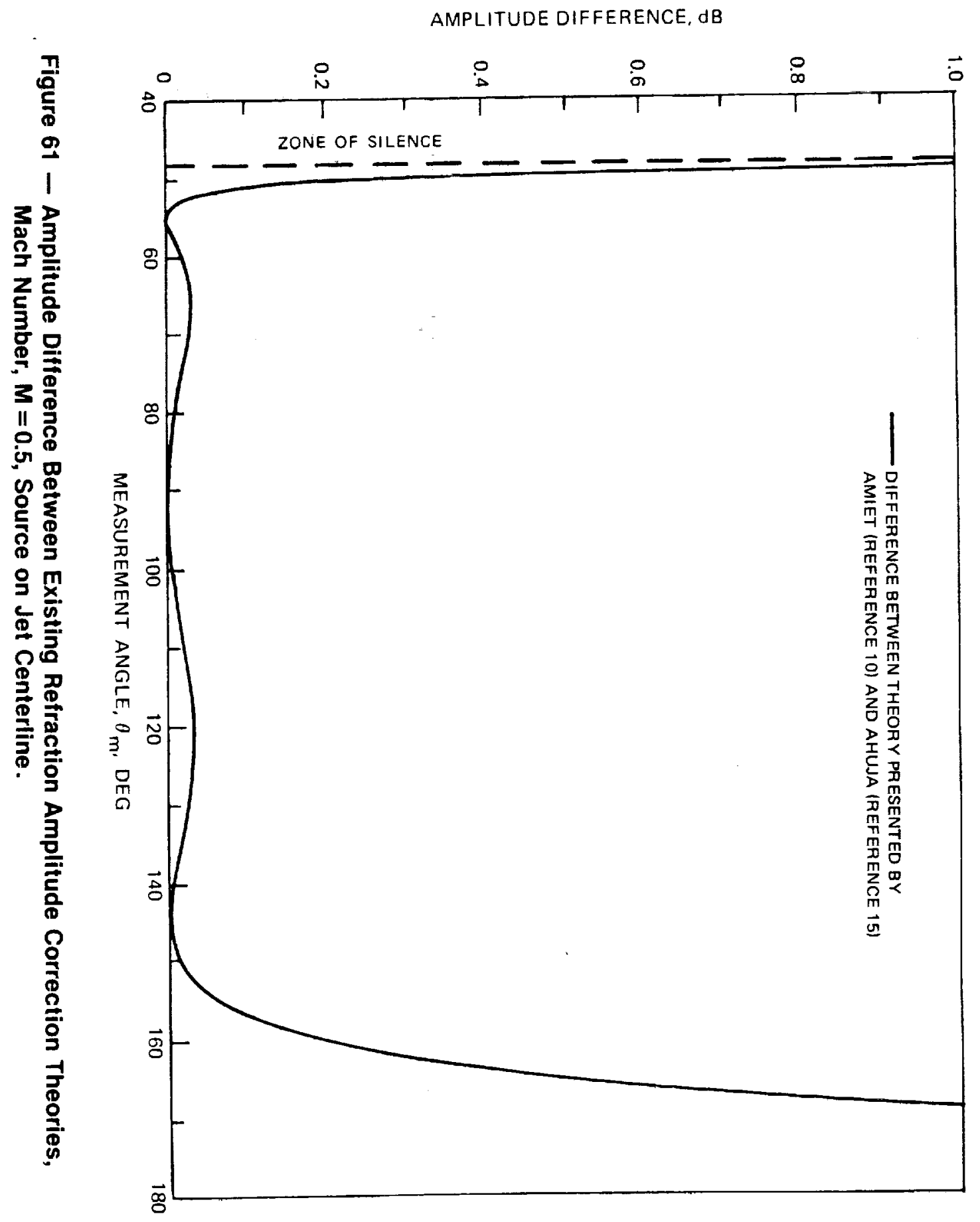


$\Xi$

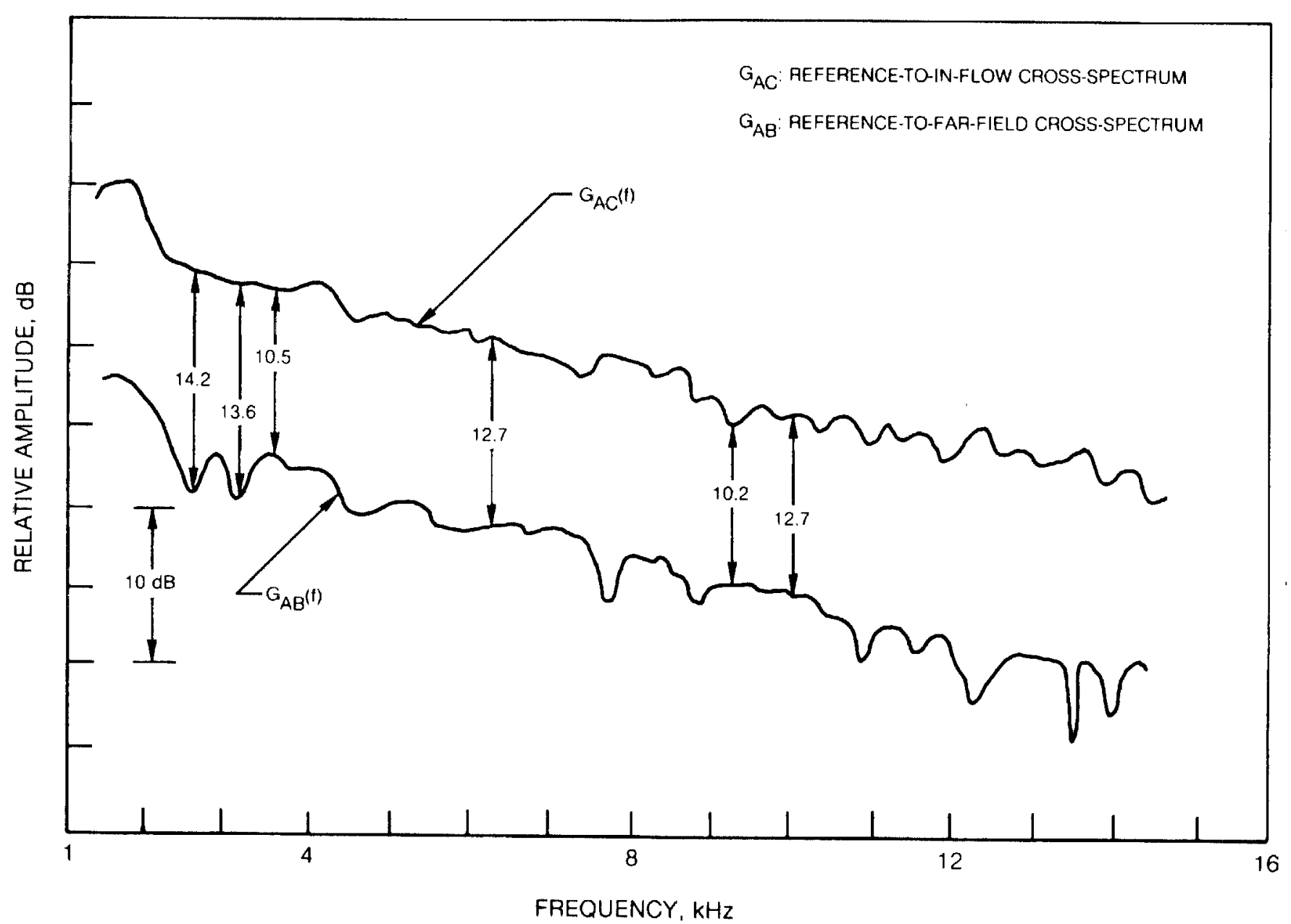

Figure 62 - Cross.Power Spectra, $\theta_{C}=90^{\circ}, X / R_{O}=4, M=0.225$. 

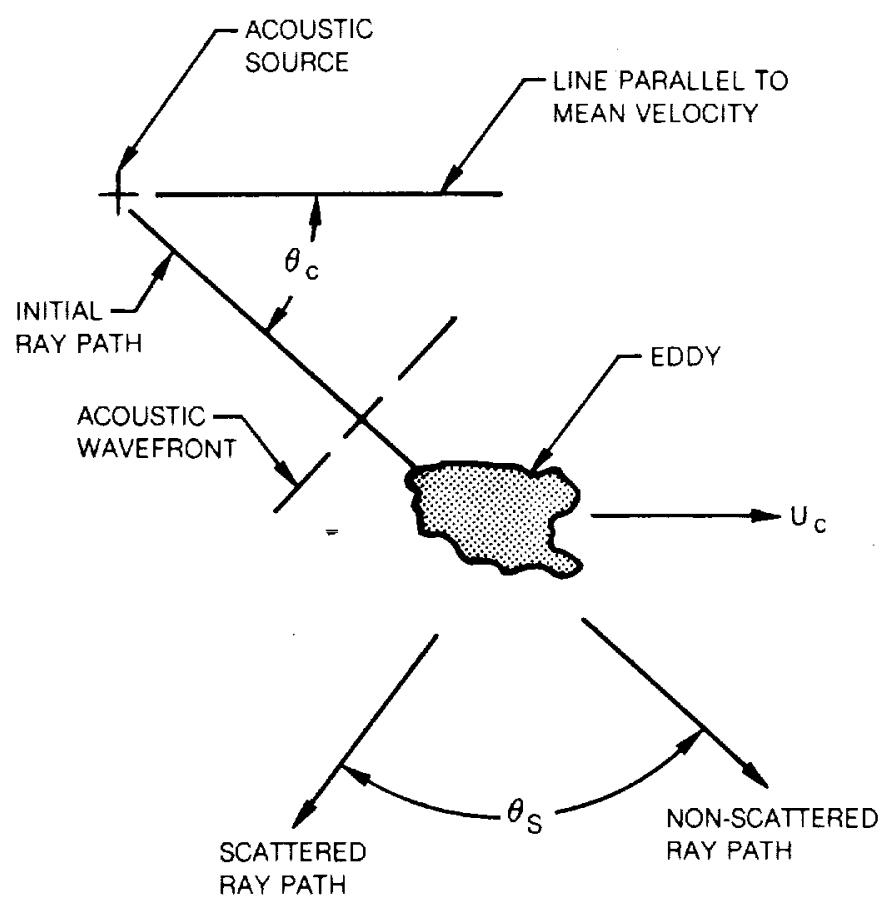

Figure $63 a-$ Schematic of Direction Scattering by Turbulent Eddy. 


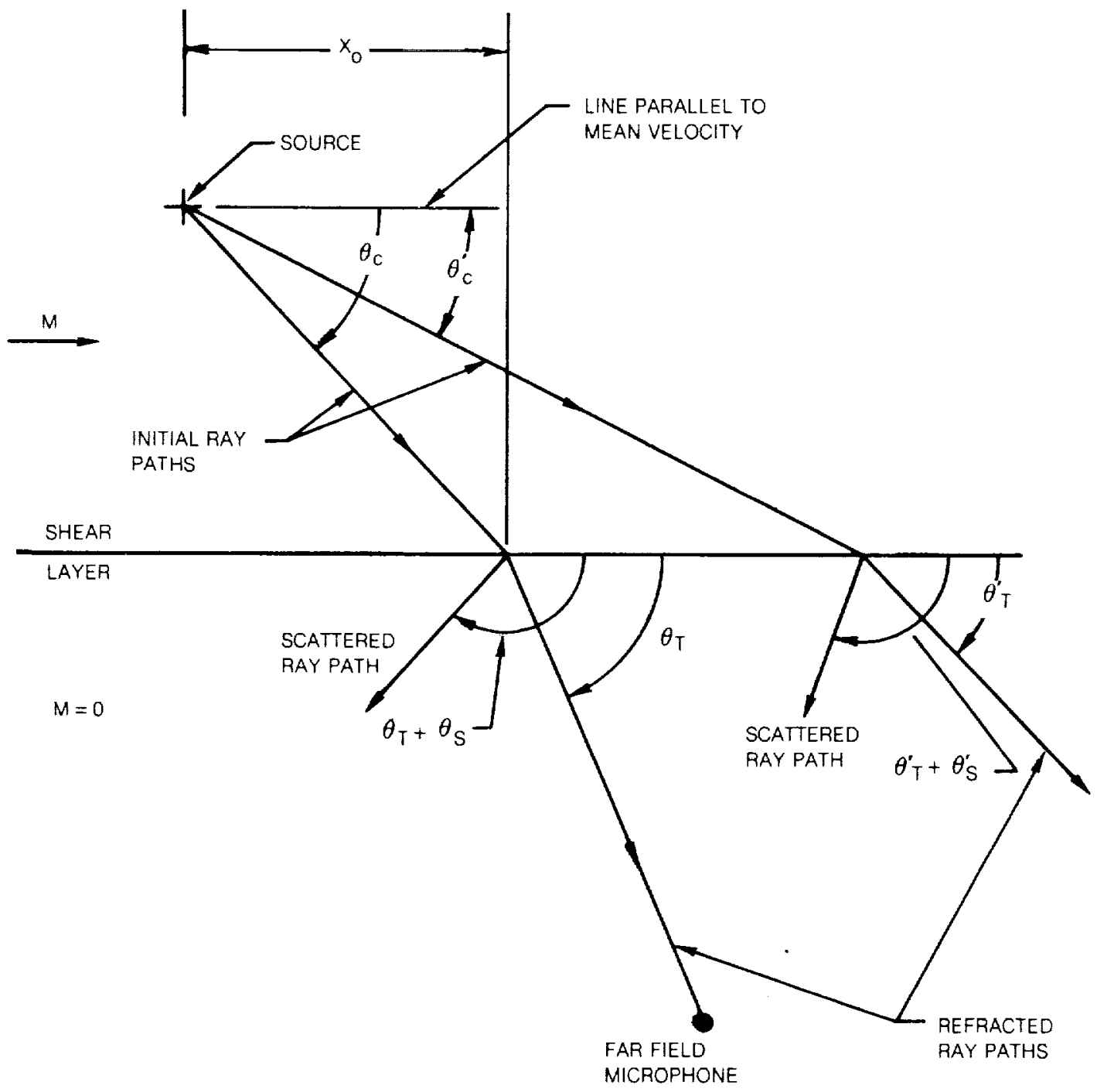

Flgure 63b - Schematic Showing Refraction Angle Change and Scattering by Different Regions of Shear Layer. 


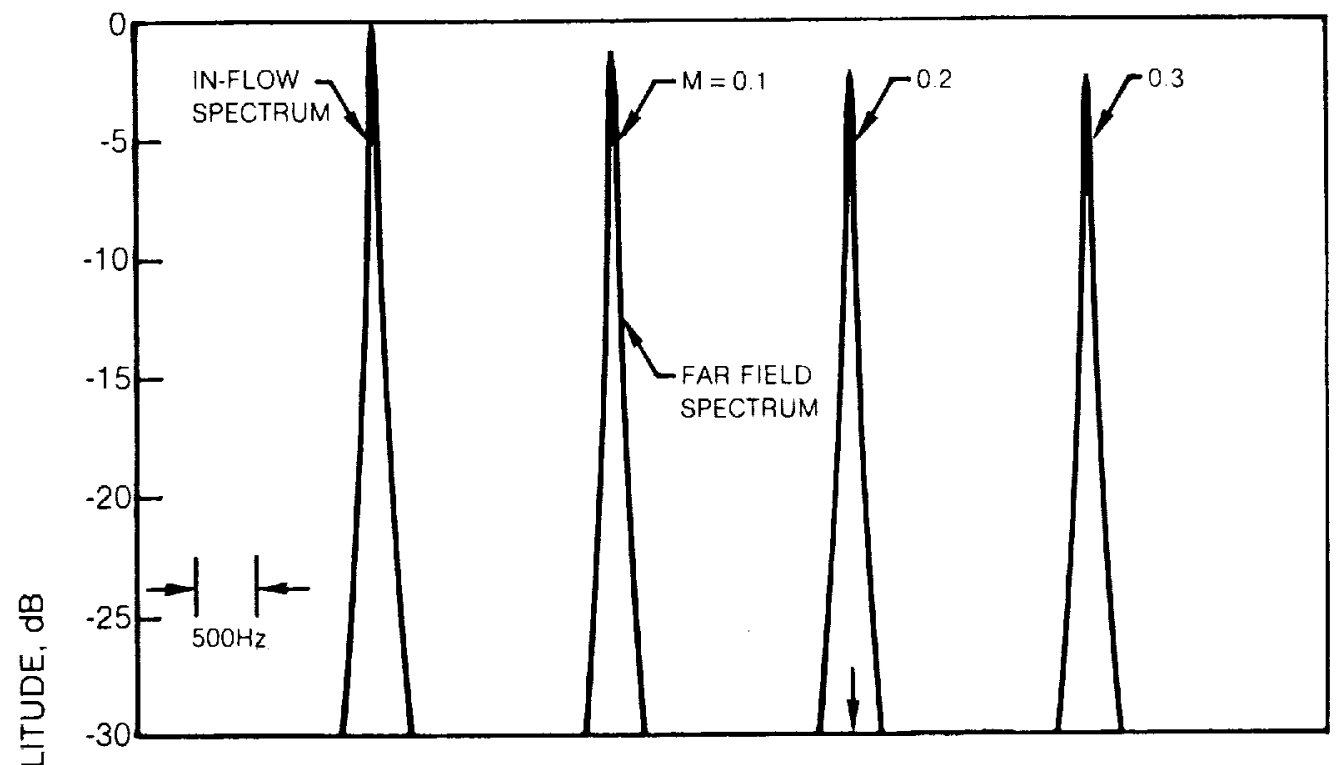

a) $f=5 \mathrm{kHz}$

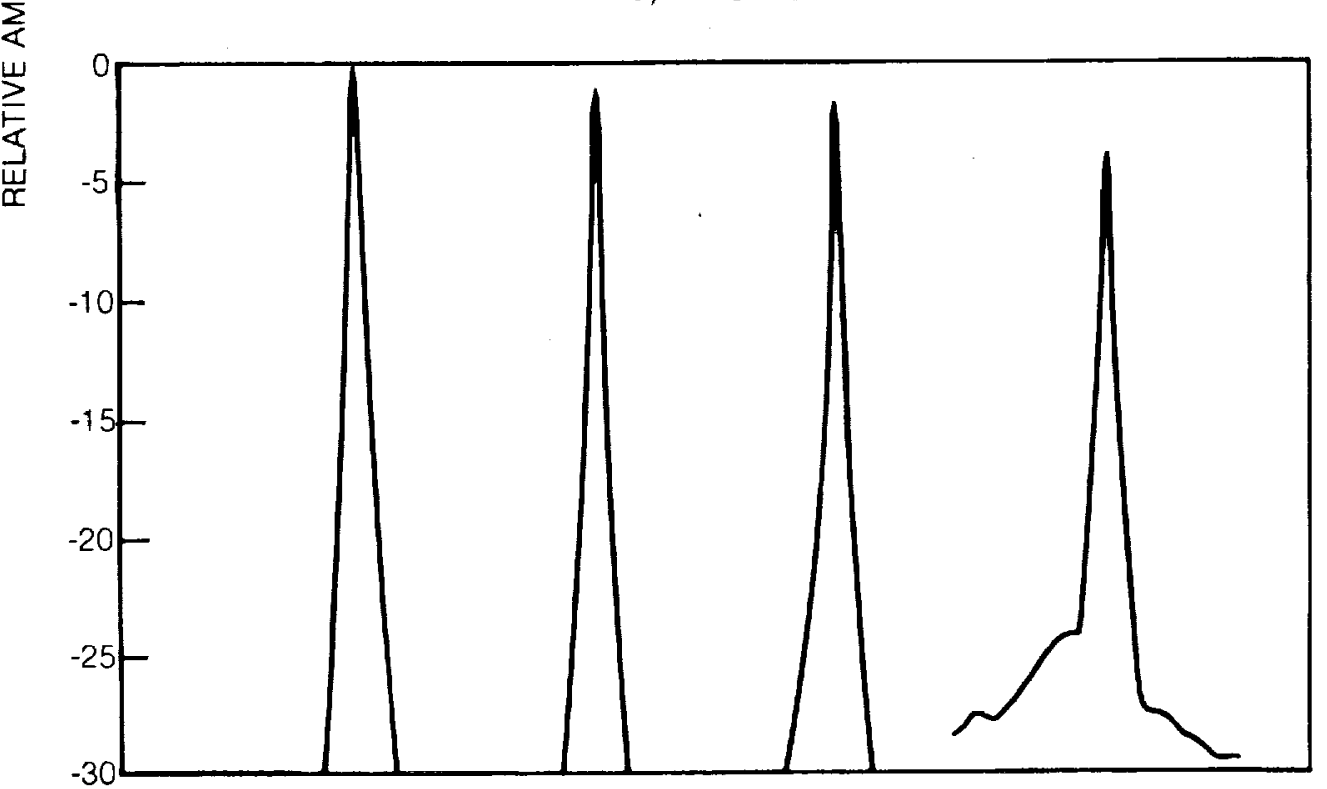

b) $f=10 \mathrm{kHz}$

Figure 64 - Variation of Discrete Tone Spectrum with Mach Number and Frequency for $\theta_{c}=90^{\circ}, X / R_{0}=0.53, B W=50 \mathrm{~Hz}$, Test

Configuration 9. 


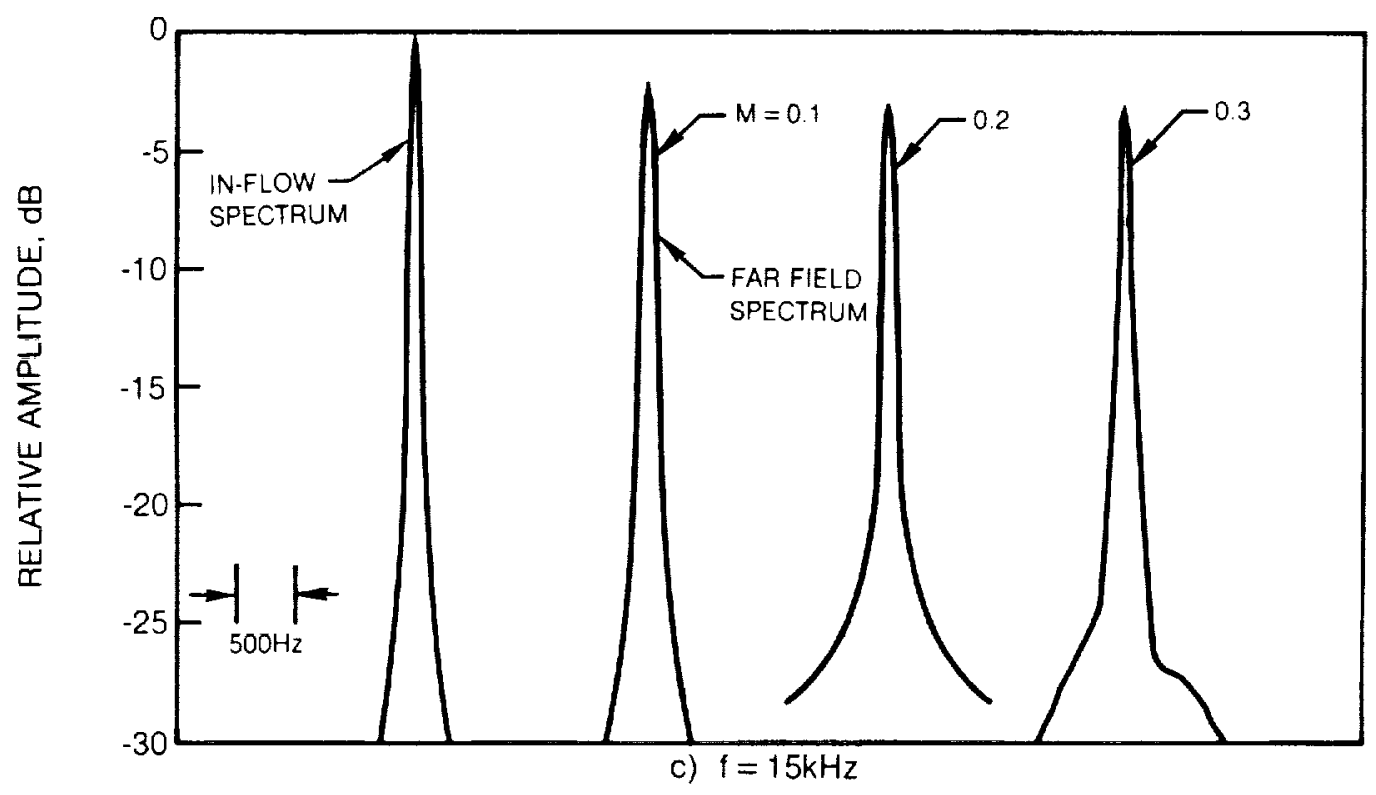

Figure 64 - Concluded. 


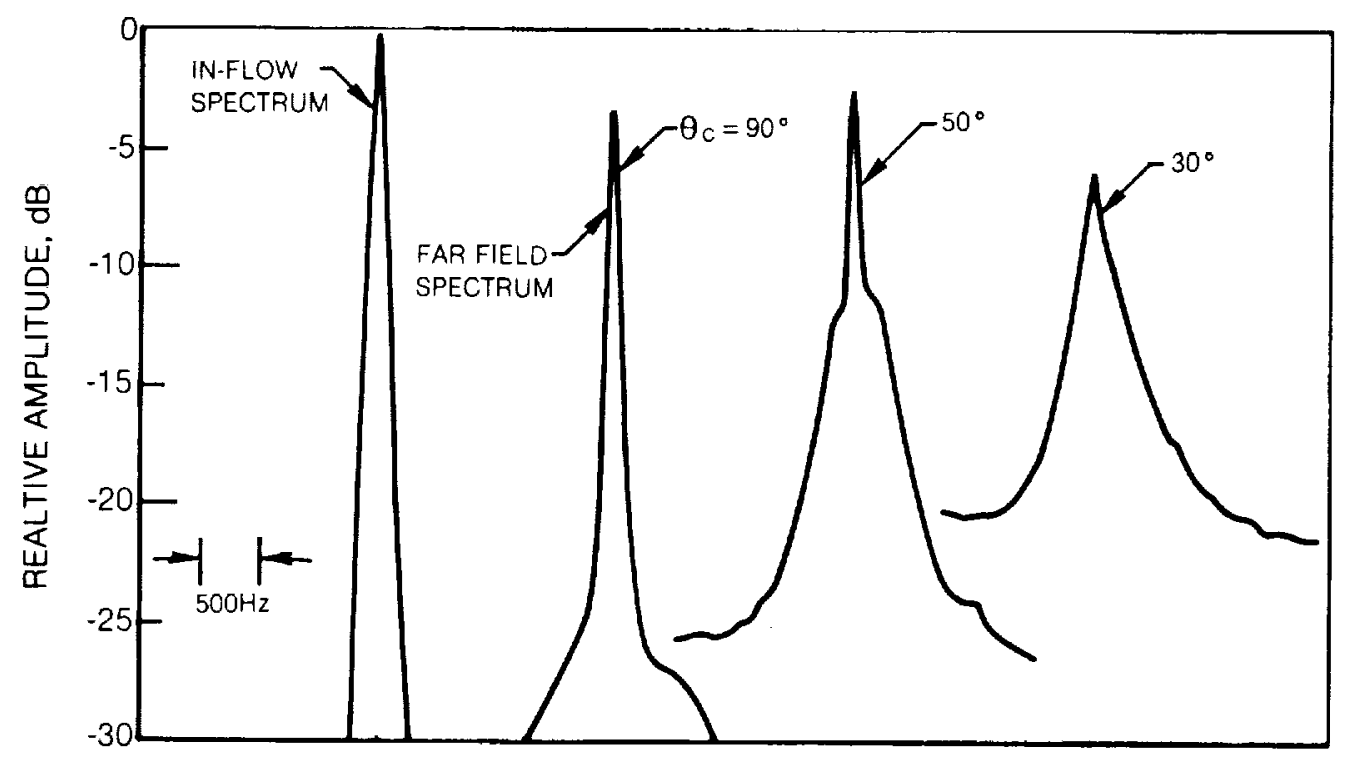

Figure 65 - Variation of Discrete Tone Spectrum with Radiation Angle for $f=15 \mathrm{kHz}, X / R_{0}=0.53, M=0.3, B W=50 \mathrm{~Hz}$, Test Configuration 9 . 


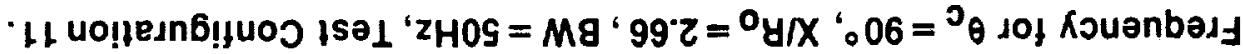

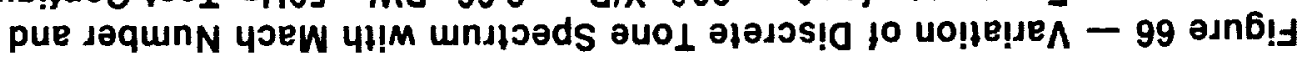
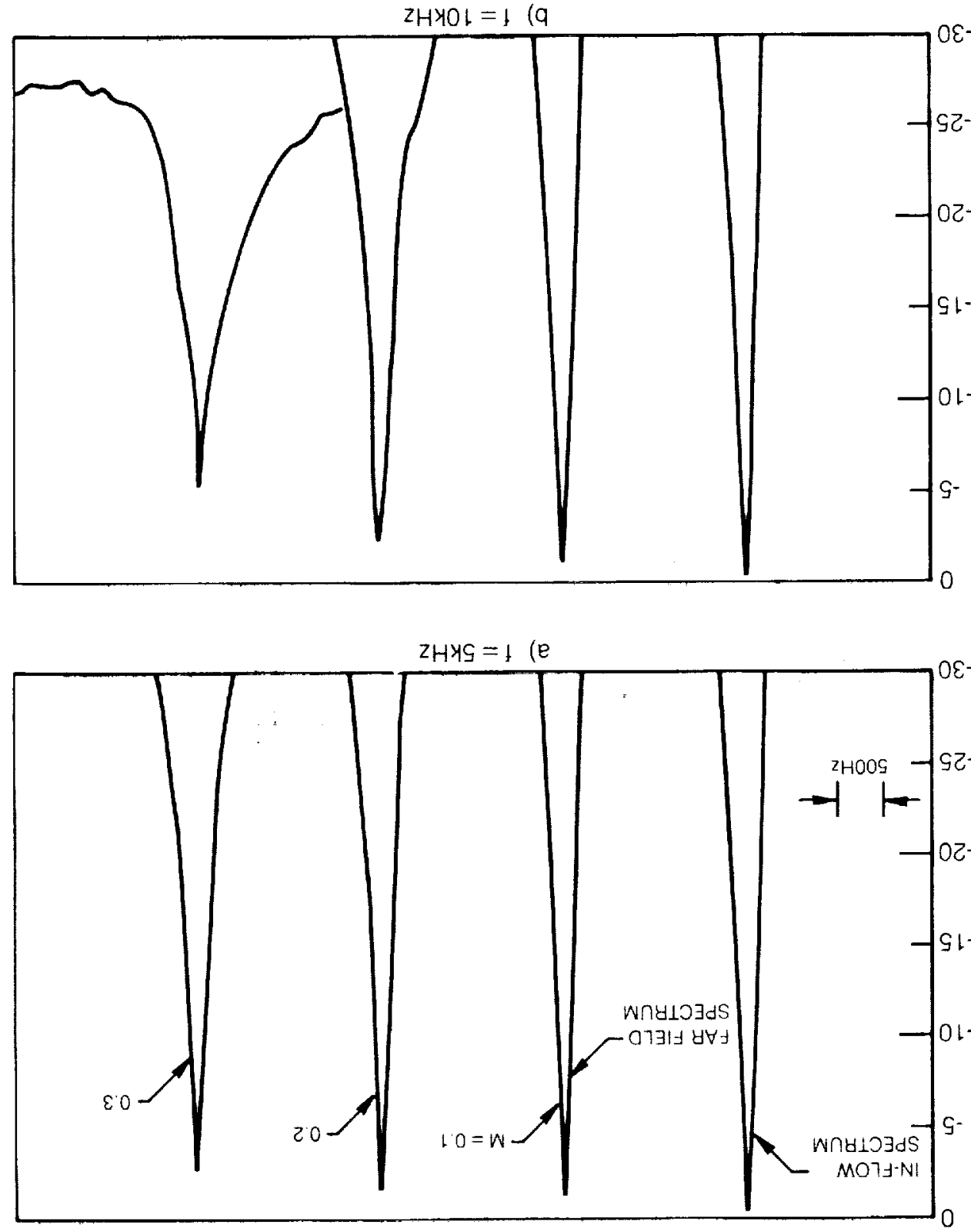


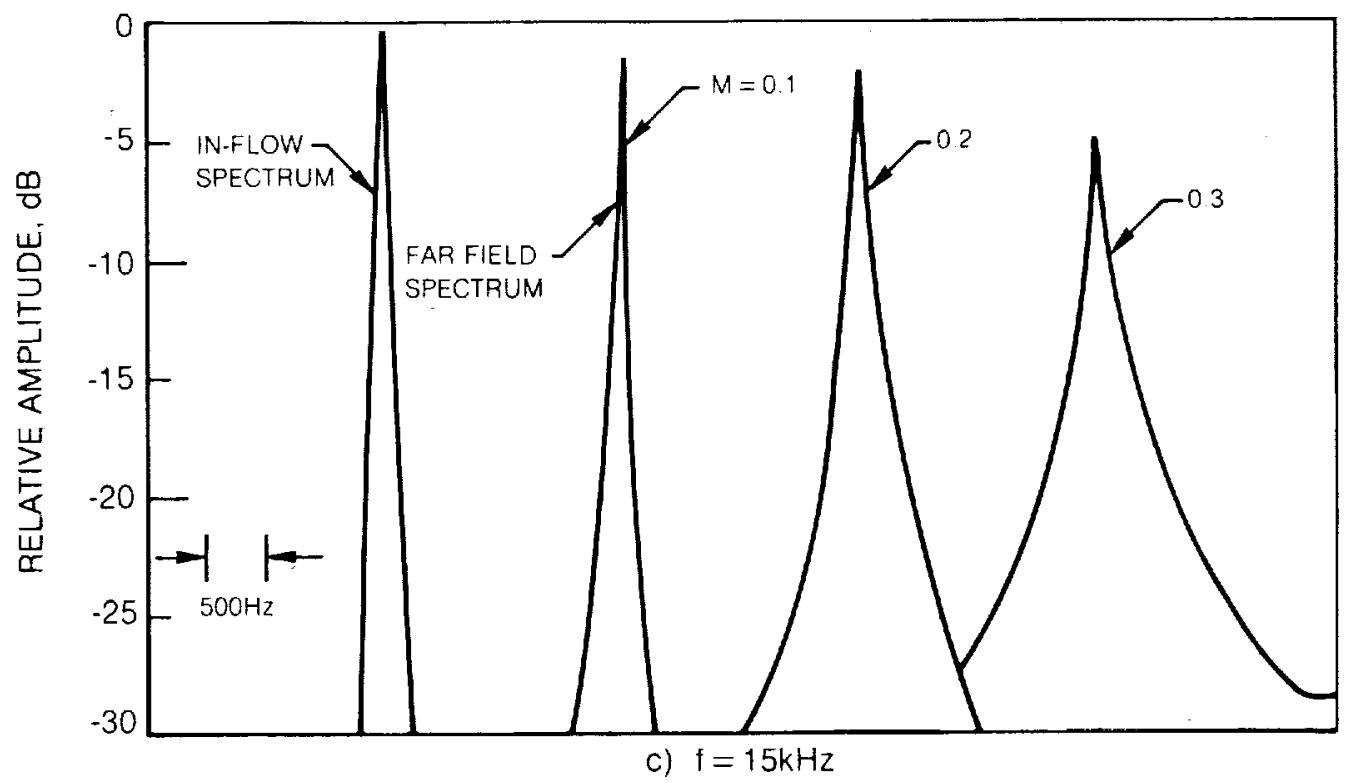

Figure 66 - Concluded. 


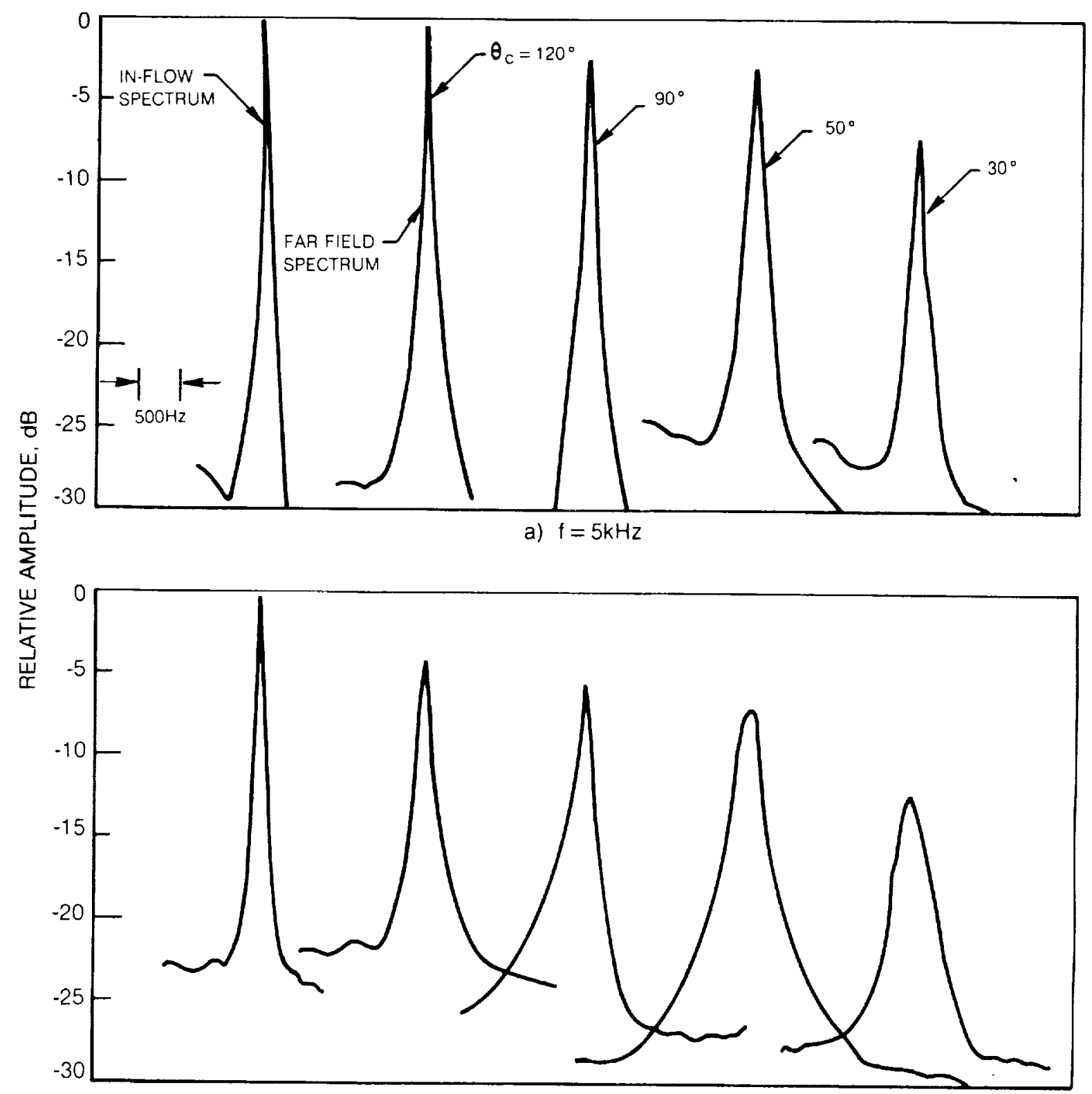

b) $f=10 \mathrm{kHz}$

Figure 67 - Variation of Discrete Tone Spectrum with Radiation Angle and Frequency for $M=0.3, X / R_{0}=2.66, B W=50 \mathrm{~Hz}$, Test Configuration 11 . 


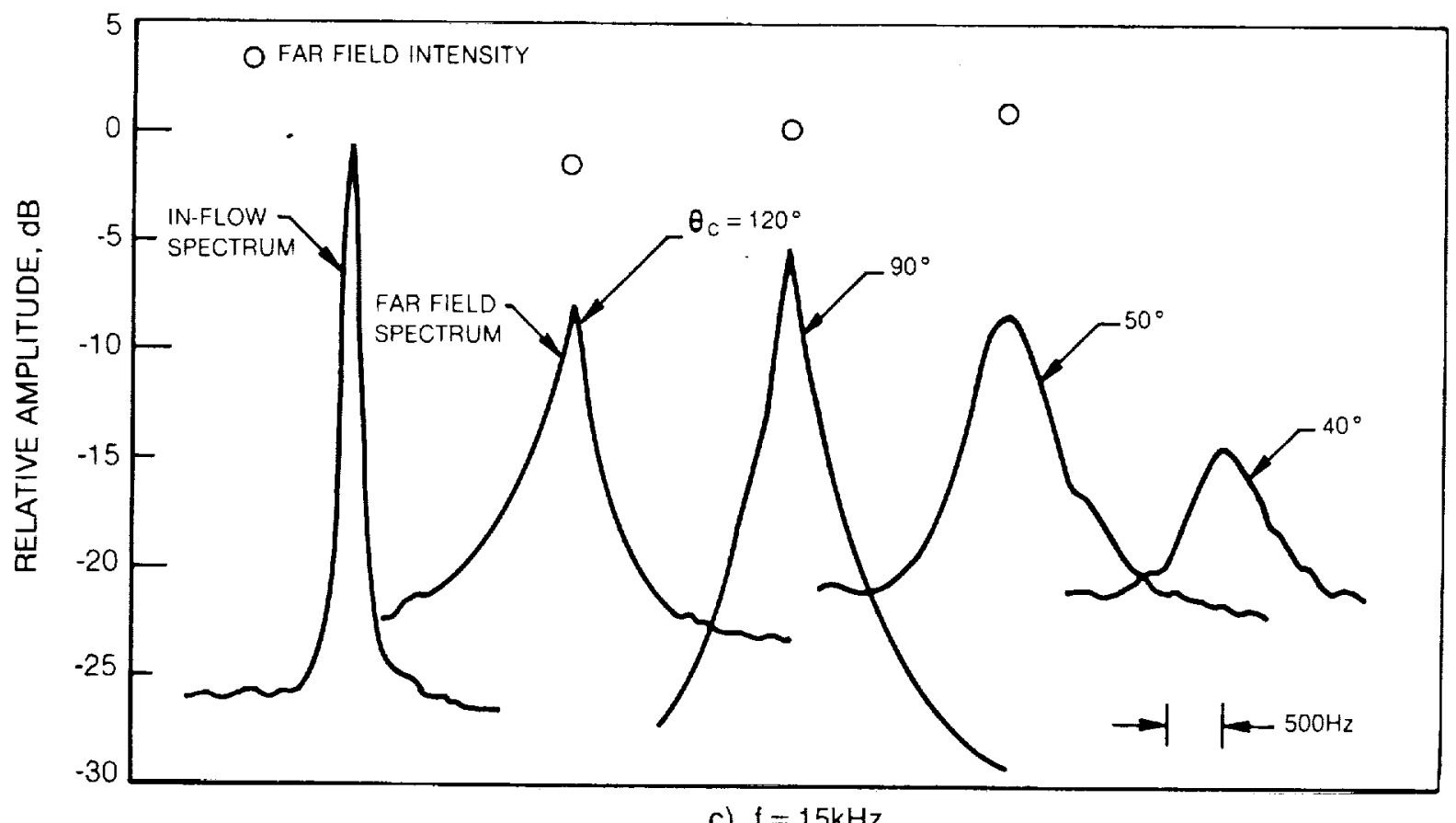

c) $f=15 \mathrm{kHz}$

Figure 67 - Concluded. 


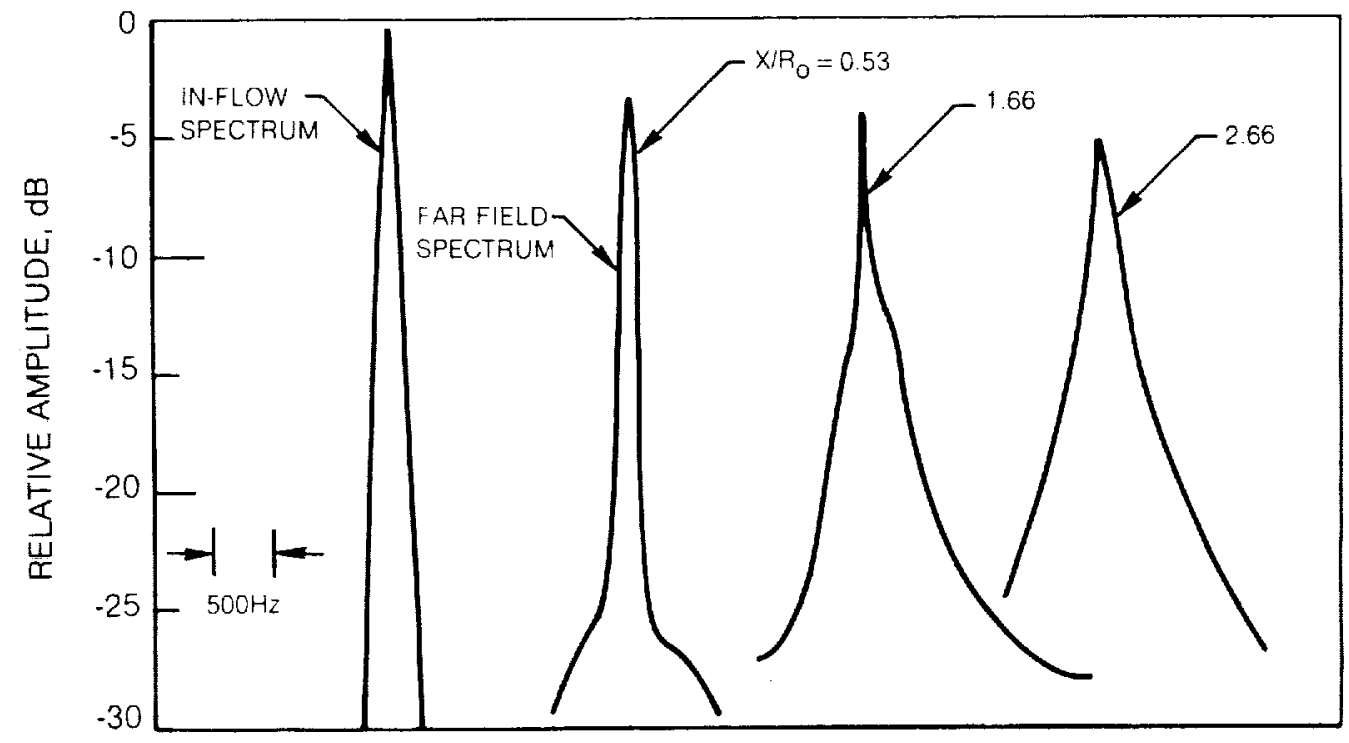

Figure 68 - Variation of Discrete Tone Spectrum with Axial Source Location for $\theta_{C}=90^{\circ}, f=15 \mathrm{kHz}, M=0.3, \mathrm{BW}=50 \mathrm{~Hz}$. 


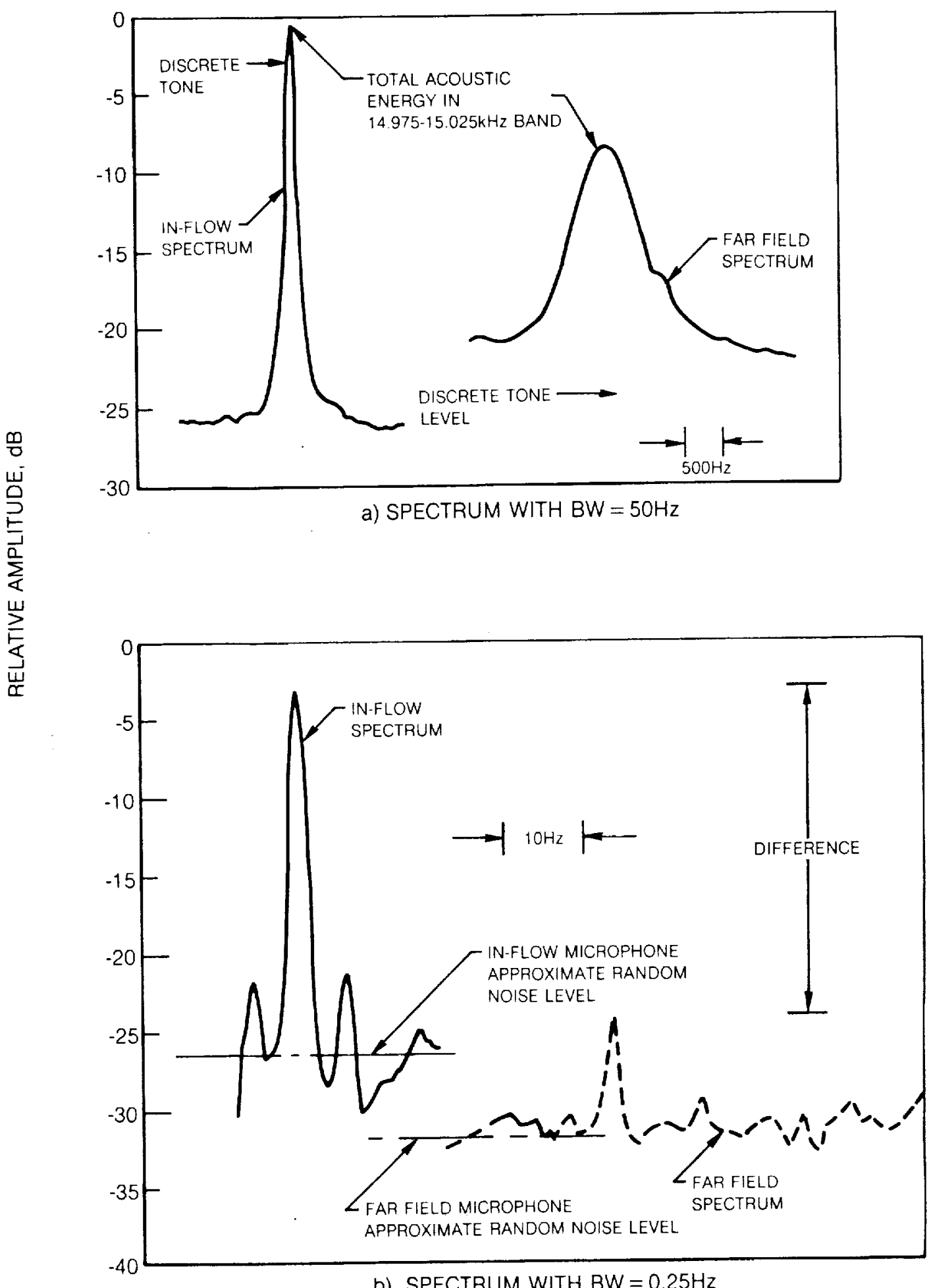

Figure 69 - Comparison of Spectra Obtained with Different Analyzer Bandwidths for $\theta_{C}=50^{\circ}, X / R_{0}=2.66, f=15 \mathrm{kHz}, M=0.3$, Test Configuration 11 . 


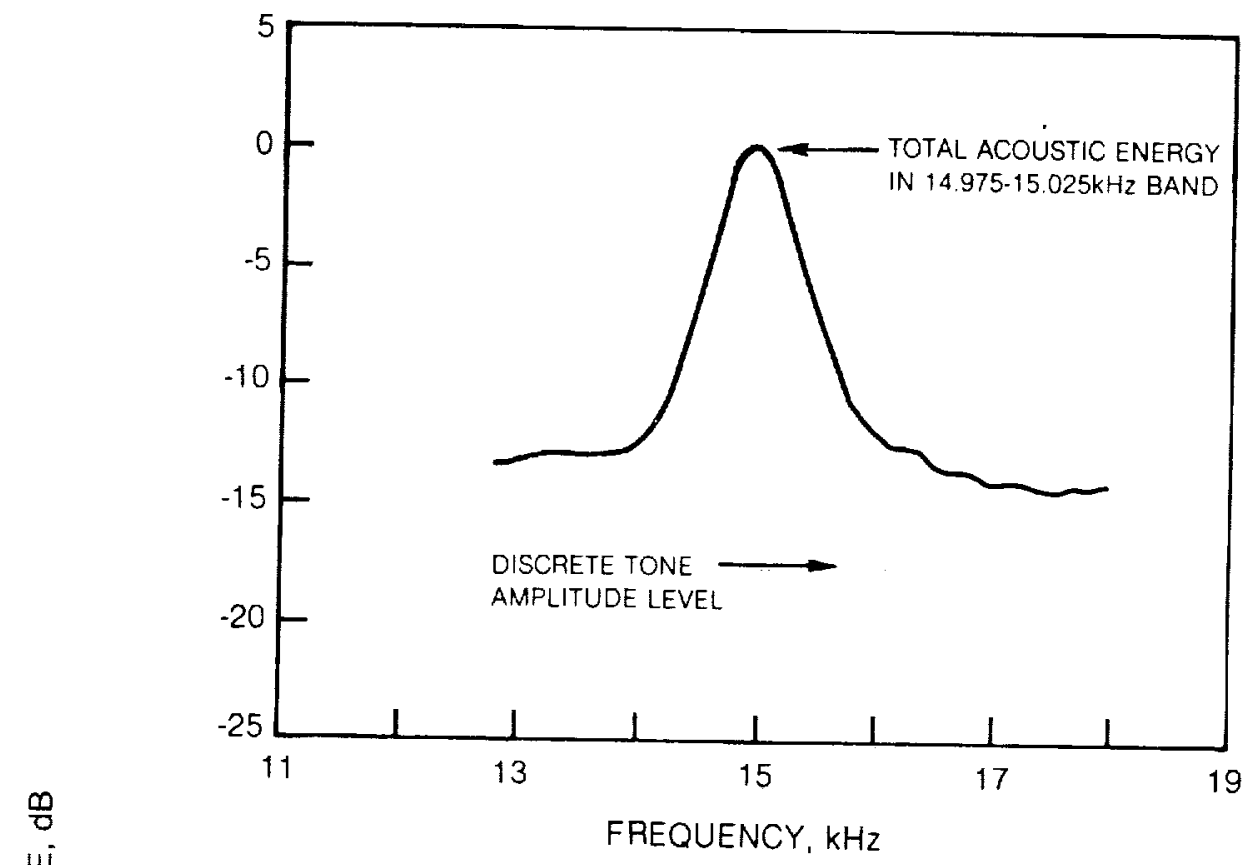

a) SPECTRUM FOR $f=15 \mathrm{kHz}, \mathrm{BW}=50 \mathrm{~Hz}$

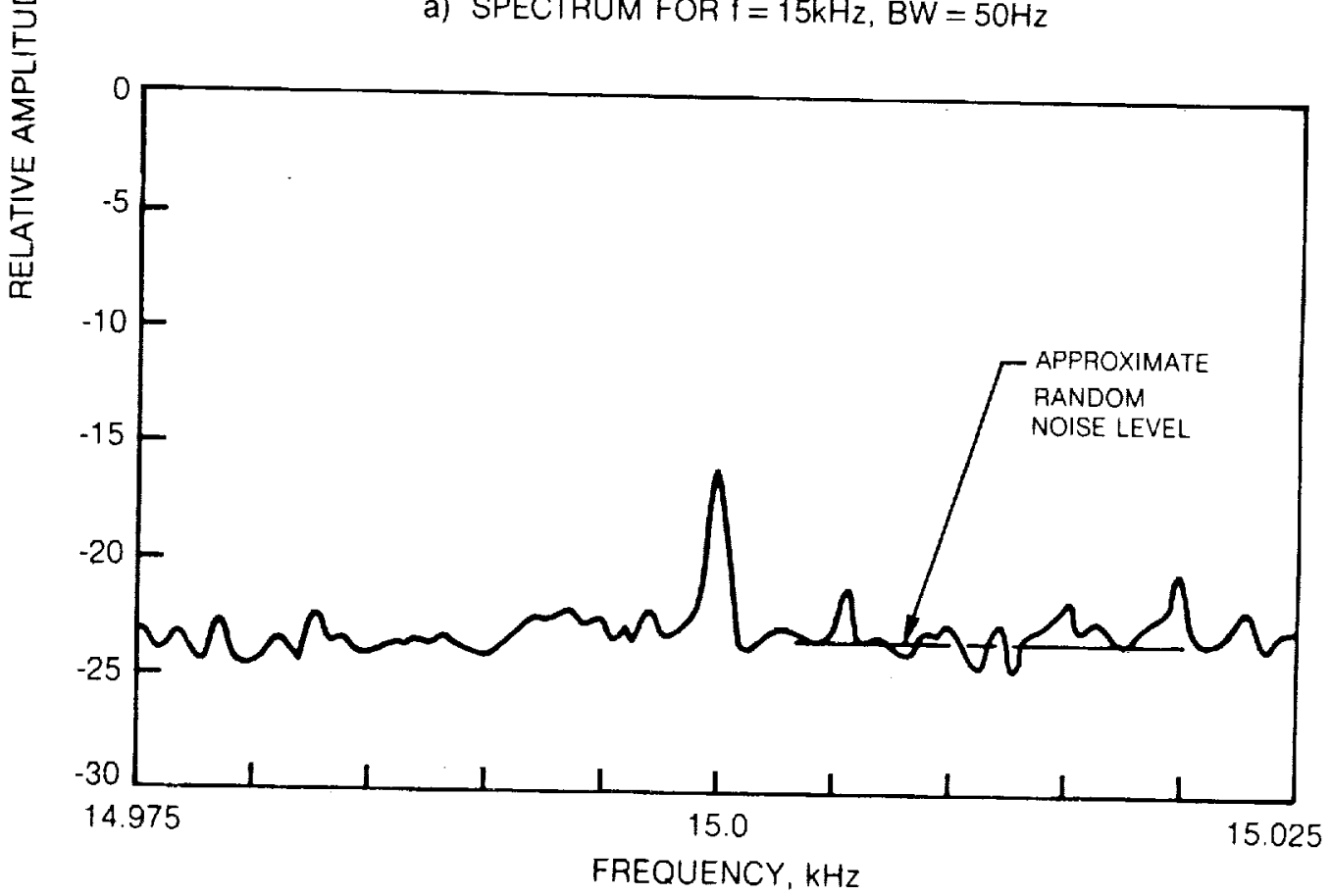

b) SPECTRUM FOR $f=15 \mathrm{kHz}, B W=0.25 \mathrm{~Hz}$

Figure 70 - Far Field Microphone Spectra Obtained with Different Bandwidths for $\theta_{C}=50^{\circ}, X / R_{O}=2.66, M=0.3$, Test Configuration 11 . 


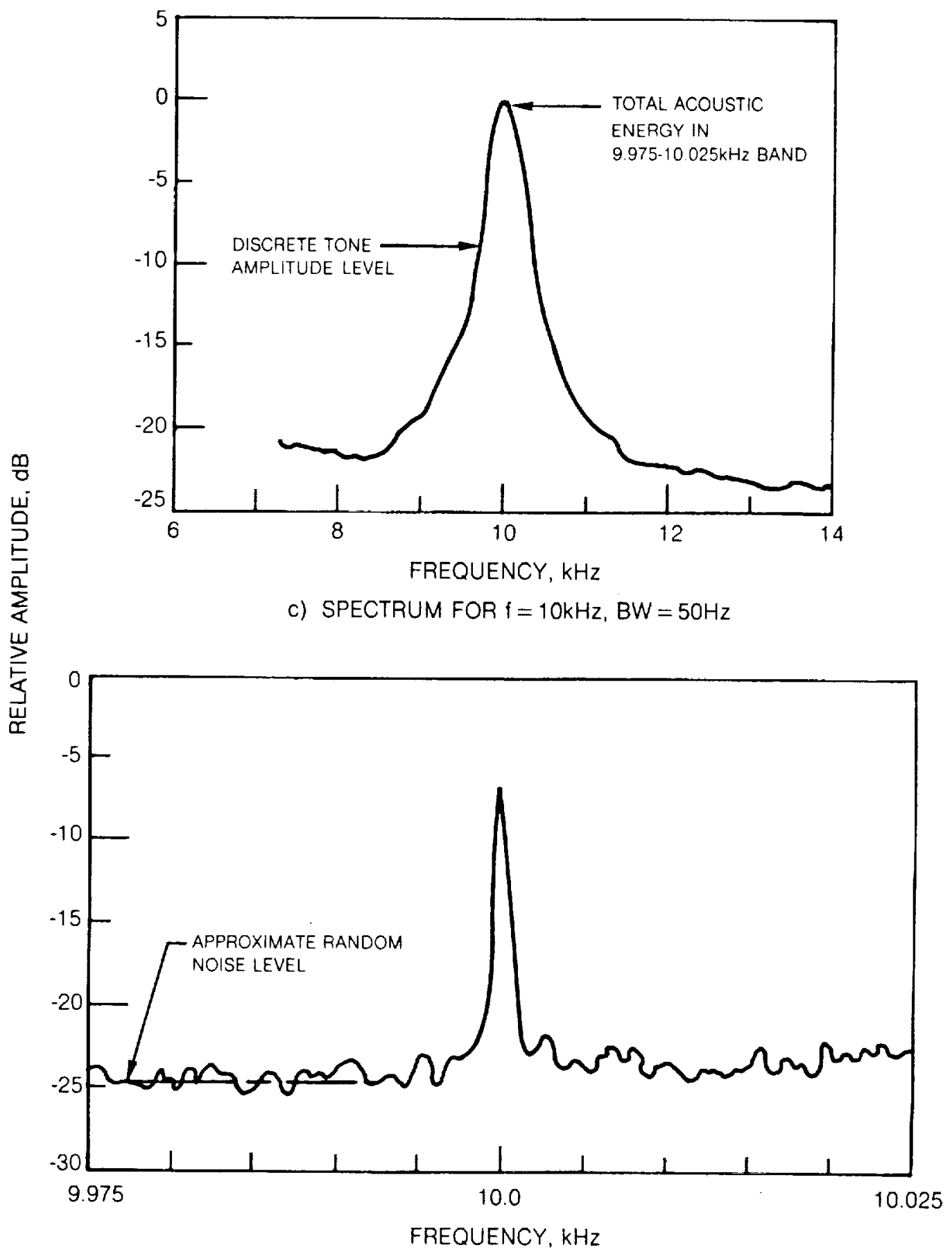

d) SPECTRUM FOR $\mathrm{f}=10 \mathrm{kHz}, \mathrm{BW}=0.25 \mathrm{~Hz}$

Figure $70-$ Continued. 


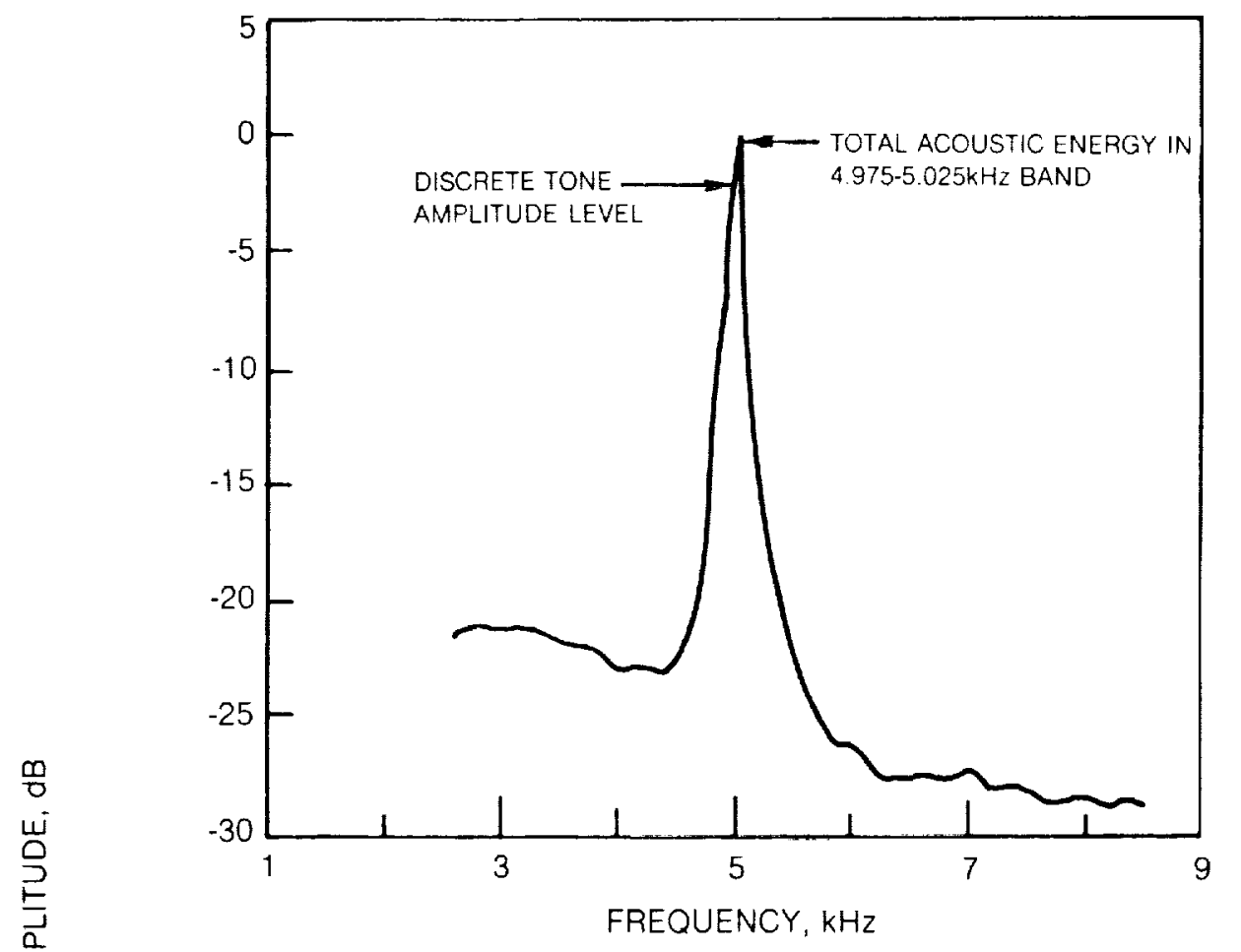

e) SPECTRUM FOR $\mathrm{f}=5 \mathrm{kHz}, \mathrm{BW}=50 \mathrm{~Hz}$

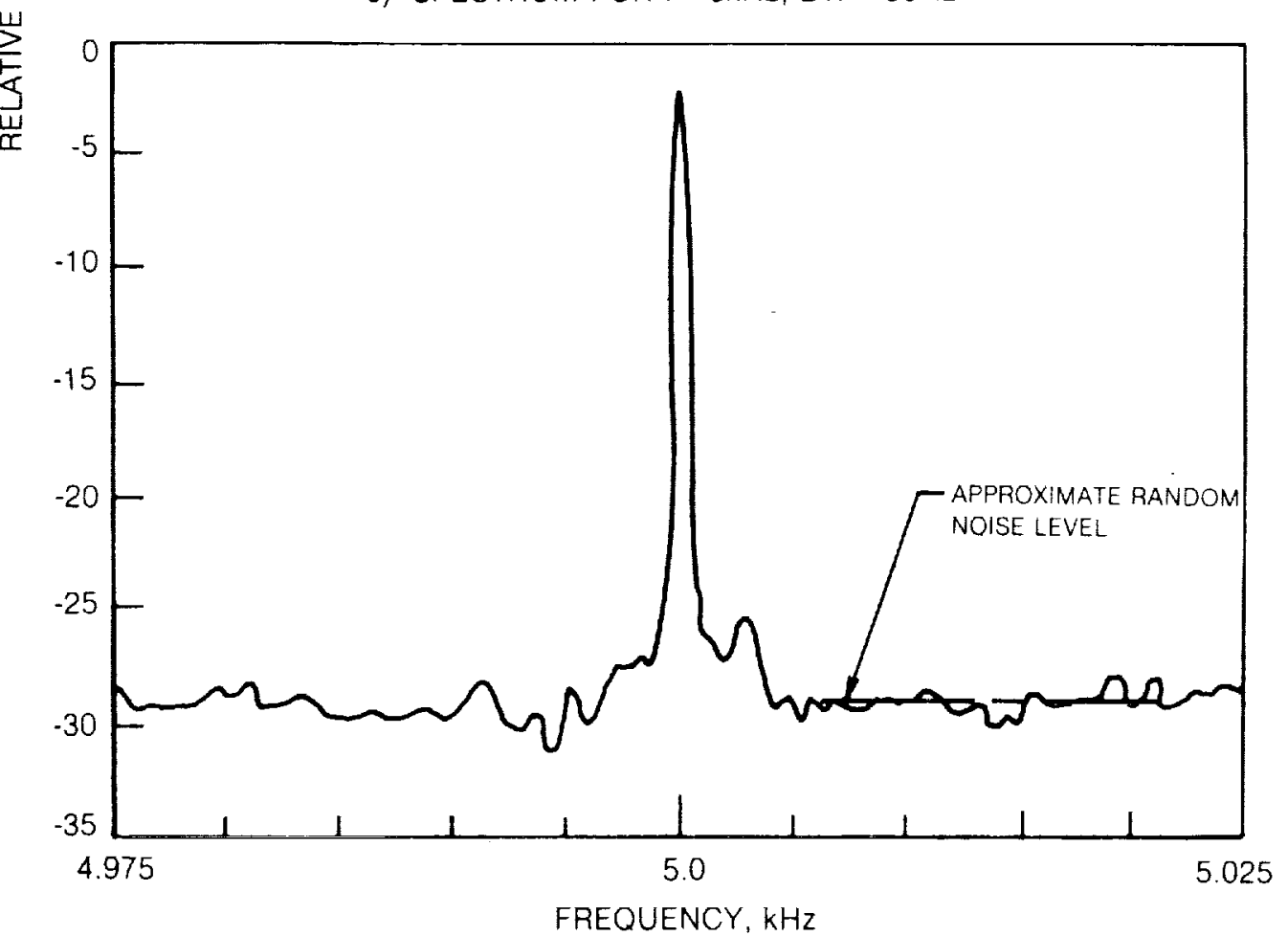

f) SPECTRUM FOR $\mathrm{f}=5 \mathrm{kHz}, B W=0.25 \mathrm{~Hz}$

Figure 70 - Concluded. 


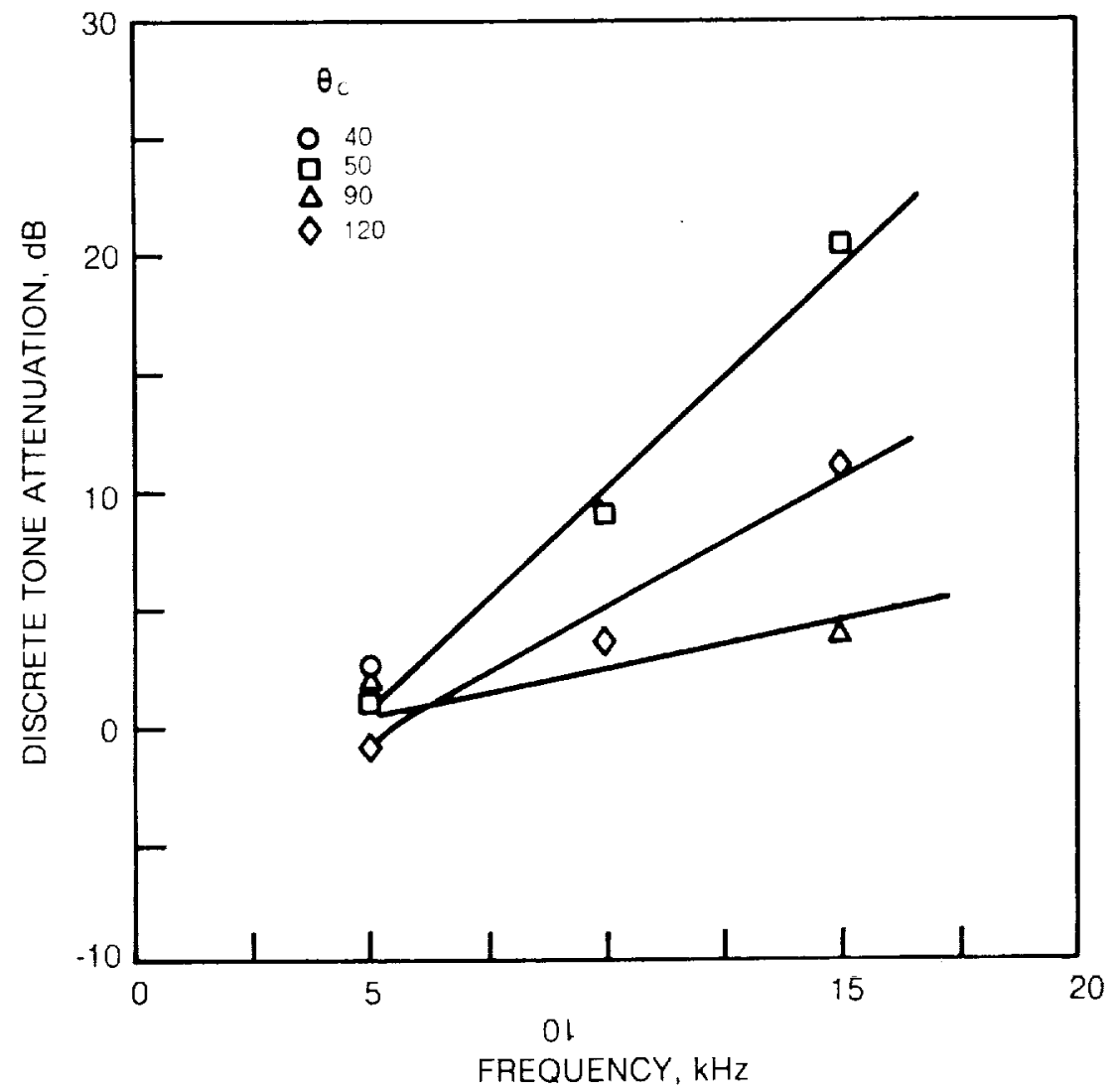

Figure 71 - Discrete Tone Attenuation as a Function of Frequency and Radiation Angle for $X / R_{0}=2.66, M=0.3, B W=0.25 H z$, Test Configuration 11 . 


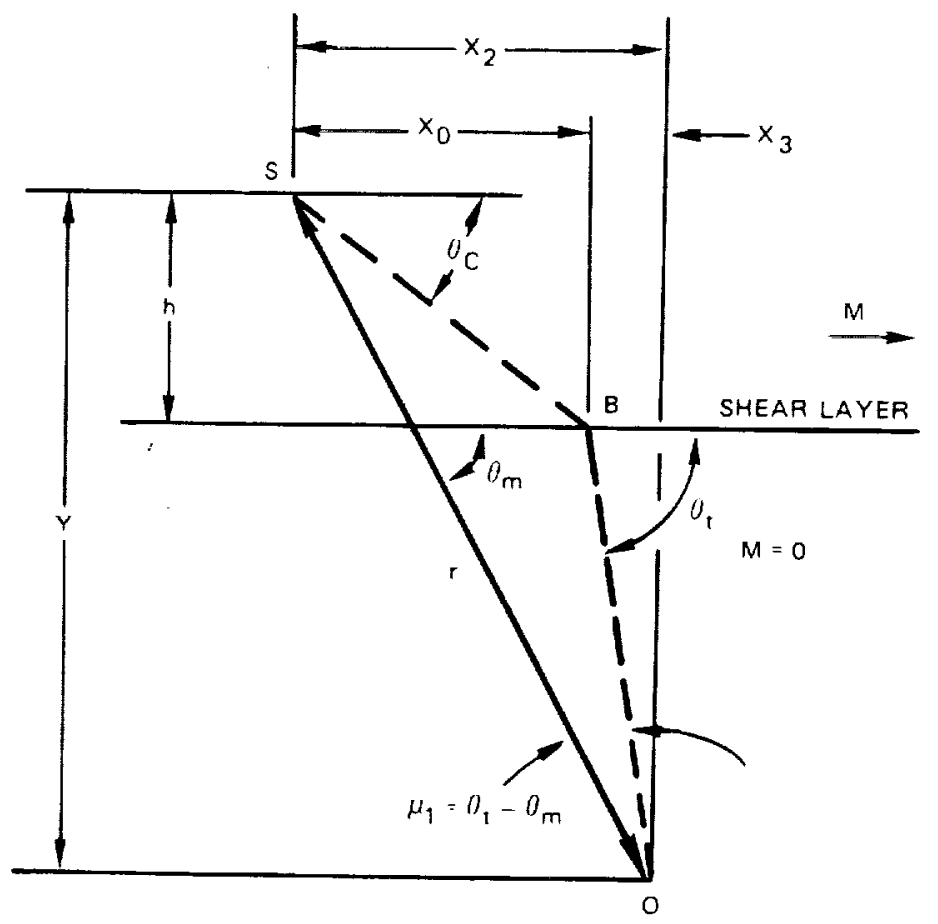

Figure 72 - Geometry for Angle Correction Calculation. 


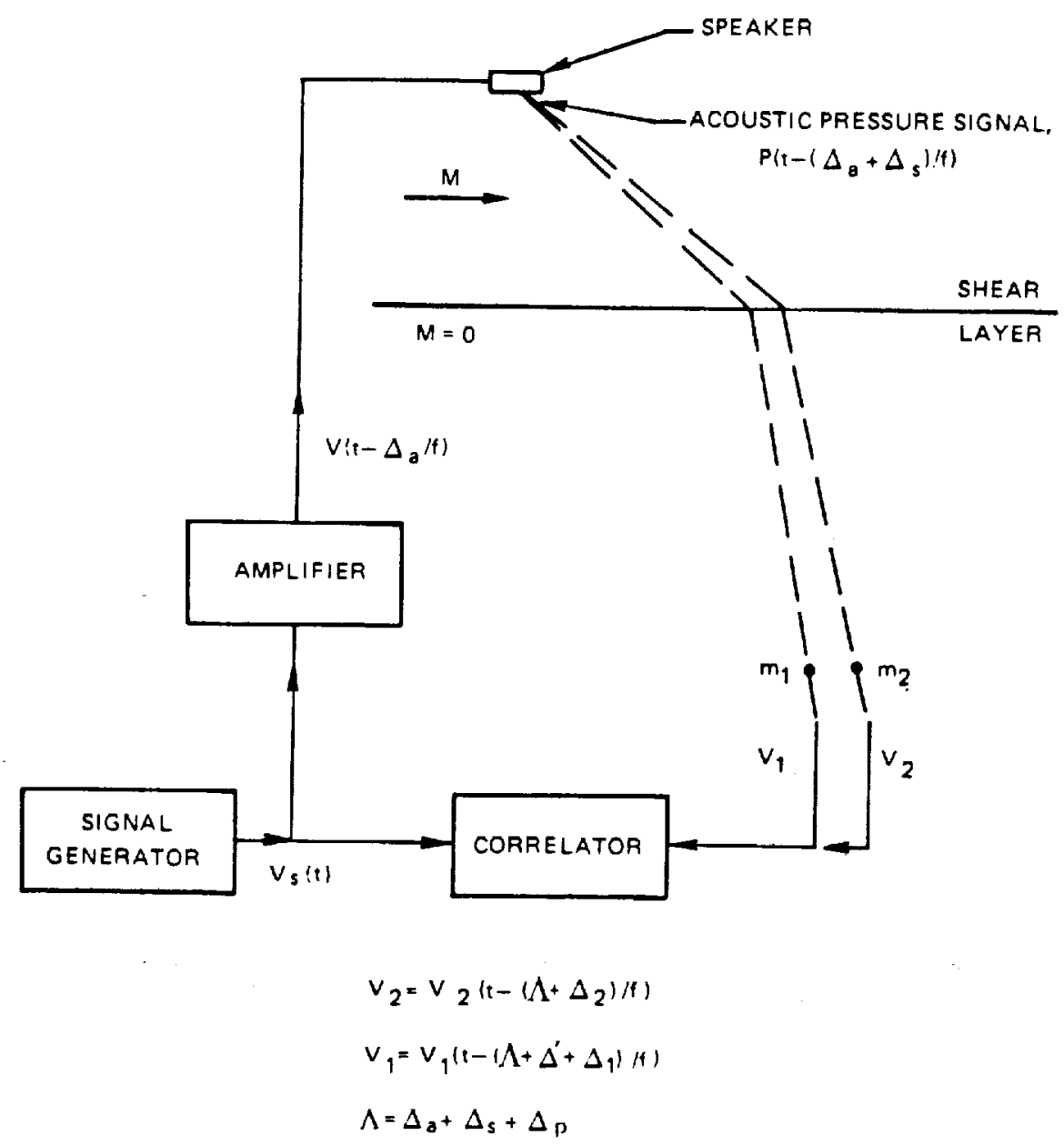

Fig. 73 - Schematic Showing Phase Lag Between Signal Generator and Far.Field Microphone Output. 


\begin{tabular}{|c|c|c|c|c|}
\hline $\begin{array}{l}\text { 1. Report No. } \\
\text { NASA CR-3371 }\end{array}$ & \multicolumn{2}{|c|}{ 2. Government Accession No. } & \multicolumn{2}{|c|}{ 3. Recipient's Catalog No. } \\
\hline \multicolumn{3}{|l|}{ 4. Title and Subtitle } & \multicolumn{2}{|c|}{$\begin{array}{l}\text { 5. Report Date } \\
\text { December } 1980\end{array}$} \\
\hline \multicolumn{3}{|c|}{ REFRACTION AND SCATTERING OF SOUND BY A SHEAR LAYER } & \multicolumn{2}{|c|}{ 6. Performing Organization Code } \\
\hline \multicolumn{3}{|c|}{$\begin{array}{l}\text { 7. Author(s) } \\
\text { Robert H. Schlinker and Roy K. Amiet }\end{array}$} & \multicolumn{2}{|c|}{ 8. Performing Organization Report No. } \\
\hline \multirow{3}{*}{\multicolumn{3}{|c|}{$\begin{array}{l}9 \text { Performing Organization Name and Address } \\
\text { Uni ted Technologies Research Center } \\
\text { East Hartford, Connecticut } 06108\end{array}$}} & \multicolumn{2}{|c|}{ 10. Work Unit No. } \\
\hline & & & \multicolumn{2}{|c|}{$\begin{array}{l}\text { 11. Contract or Grant No. } \\
\text { NAS1-15339 }\end{array}$} \\
\hline & & & \multicolumn{2}{|c|}{$\begin{array}{l}\text { 13. Type of Report and Period Covered } \\
\text { Contractor Report }\end{array}$} \\
\hline \multicolumn{3}{|c|}{$\begin{array}{l}\text { 12. Sponsoring Agency Name and Address } \\
\text { National Aeronautics and Space Administration } \\
\text { Washington, D.C. } 20546\end{array}$} & \multicolumn{2}{|c|}{ 14. Sponsoring Agency Code } \\
\hline \multicolumn{5}{|c|}{$\begin{array}{l}\text { 15. Supplementary Notes } \\
\text { Langley Technical Monitor: James C. Yu } \\
\text { Final Report }\end{array}$} \\
\hline \multicolumn{5}{|c|}{$\begin{array}{l}\text { 16. Abstract } \\
\text { A theoretical and experimental study was conducted to determine the angle and amplitude } \\
\text { changes for acoustic waves refracted by a circular open-jet shear layer. The general- } \\
\text { ized refraction theory was assessed experimentally for on-axis and off-axis acoustic } \\
\text { source locations as source frequency varied from } 1 \mathrm{kHz} \text { to } 10 \mathrm{kHz} \text { and free stream Mach } \\
\text { number varied from } 0.1 \text { to } 0.4 \text {. Angle and amplitude changes across the shear layer } \\
\text { showed good agreement with theory. Experiments confirmed that the refraction theory } \\
\text { is independent of shear layer thichess, acoustic source frequency, and source type. } \\
\text { A generalized theory is, thus, avajlable for correcting far field noise data acquired } \\
\text { in open-jet test facilities. } \\
\text { A theoretical and experimental study was also conducted to investigate the effect of } \\
\text { discrete tone scattering by the open-jet turbulent shear layer. Scattering effects } \\
\text { were investigated over the same Mach number range as frequency varied from } 5 \text { kHz to } \\
\text { l5 kHz. Attenuation of discrete tone amplitude and tone boradening were measured as a } \\
\text { function of acoustic source position and radiation angle. Scattering was found to be } \\
\text { stronger at angles close to the open jet axis than at } 90^{\circ} \text {. In addition, scattering } \\
\text { becomes stronger as the acoustic source position shifts downstream. Thus, corrections } \\
\text { for discrete tone amplitude changes across the shear layer must account for both } \\
\text { refraction and scattering amplitude changes. A scattering analysis provided an estimate } \\
\text { of the onset of discrete tone scattering. }\end{array}$} \\
\hline \multicolumn{2}{|c|}{$\begin{array}{l}\text { 17. Key Words (Suggested by Author(s)/ } \\
\text { Refraction of Sound } \\
\text { Scattering of Sound } \\
\text { Sound Propagation Through Shear Layer } \\
\text { Shear Layers } \\
\text { Turbulence Scattering } \\
\end{array}$} & \multicolumn{3}{|c|}{$\begin{array}{r}\text { 18. Distribution Statement } \\
\text { Unclassified - Unlimited } \\
\text { Subject Category } 71\end{array}$} \\
\hline $\begin{array}{l}\text { 19. Security Classif, for this report) } \\
\text { Unclassified }\end{array}$ & $\begin{array}{l}\text { 20. Security Class } \\
\text { Unclassifie }\end{array}$ & & $\begin{array}{l}\text { of Pages } \\
189\end{array}$ & 22. Price \\
\hline
\end{tabular}

For sale by the National Technical Information Service, Springfield. Virginia 22161 